\title{
Hydrodynamics of granular gases: clustering, universality and importance of subsonic convective waves
}

\author{
Dissertation \\ zur Erlangung des mathematisch-naturwissenschaftlichen \\ Doktorgrades \\ "Doctor rerum naturalium" \\ der Georg-August-Universität Göttingen \\ im Promotionsprogramm PROPHYS \\ der Georg-August University School of Science (GAUSS)
}

vorgelegt von

Mathias Hummel

aus Magdeburg

Göttingen, September 2016 


\section{Thesis committee}

\section{Prof. Dr. Stephan Herminghaus,}

Department "Dynamics of Complex Fluids", Max Planck Institute for Dynamics and Self-Organization

\section{Prof. Dr. Stefan Kehrein,}

Research Group "Condensed Matter Theory", Institute for Theoretical Physics, Physics Department, Georg-August-Universität Göttingen

Dr. Marco G. Mazza,

Research Group "Non-Equilibrium Soft Matter", Department "Dynamics of

Complex Fluids", Max Planck Institute for Dynamics and Self-Organization

\section{Thesis referees}

\section{Prof. Dr. Stephan Herminghaus,}

Department "Dynamics of Complex Fluids", Max Planck Institute for Dynamics and Self-Organization

\section{Prof. Dr. Eberhard Bodenschatz,}

Department "Fluid Dynamics, Pattern Formation and Nanobiocomplexity", Max Planck Institute for Dynamics and Self-Organization

Institute for Nonlinear Dynamics, Physics Department, Georg-August-Universität Göttingen

\section{Prof. Dr. Klaus Kassner,}

Research Group "Computational Theoretical Physics", Institute of Theoretical Physics, Physics Department, Otto-von-Guericke University Magdeburg 


\section{Examination committee}

\section{Dr. Marco G. Mazza,}

Research Group "Non-Equilibrium Soft Matter", Department "Dynamics of

Complex Fluids", Max Planck Institute for Dynamics and Self-Organization

\section{Prof. Dr. Annette Zippelius,}

Research Group "Statistical Physics and Complex Systems ", Institute for

Theoretical Physics, Physics Department, Georg-August-Universität Göttingen

\section{Prof. Dr. Stefan Kehrein,}

Research Group "Condensed Matter Theory", Institute for Theoretical Physics, Physics Department, Georg-August-Universität Göttingen

\section{Prof. Dr. Stefan Klumpp,}

Research Group "Theoretical Biophyics Group", Institute for Nonlinear Dynamics, Physics Department, Georg-August-Universität Göttingen

\section{Examination date}

26.10 .2016 
To Elisabeth and Ludwig. 


\section{Acknowledgments}

It is hard to express in words how much I appreciate my advisor Dr. Marco G. Mazza, you have been a prodigious supervisor for me. I would like to thank you for your guidance and your advices, the fruitful discussions and your fussy and critical queries which helped me in an outstanding way during my $\mathrm{PhD}$ and eventually result in my Ph.D. Thesis. Besides my advisor, I also want to thank Prof. Stephan Herminghaus for interesting discussions and giving me new ideas to my research as well as giving me the possibility to present my research on numerous conferences. I am thankful to the referees of my thesis and all the members of my thesis committee, namely Prof. Stephan Herminghaus, Prof. Eberhard Bodenschatz, Prof. Klaus Kassner, Prof. Annette Zippelius, Prof. Stefan Kehrein, Prof. Stefan Klumpp, Dr. Marco G. Mazza. Special thanks goes to Dr. James P. D. Clewett, what I know about programming is what I know from you. I thank all my collaborators namely Marius Herr, Joscha Tabet, Dr. Vinay Gupta, Artem Bolshakov and Samkit Shah. Thanks to all my present and past friends at the Max Planck Institute for Dynamics and Self-Organization for marvelous lunch discussions and enjoyable coffee breaks.

I particular want to thank my mom and my dad for their restless support. Eventually I acknowledge my partner, Franziska Rauch, and my children, Elisabeth and Ludwig, for their flawless and continuous support even at hardship. 
"All human progress always happened in the manner that a small minority began to deviate from the ideas and habits of the majority, until finally their example inspired the others to take over the new way."

Ludwig von Mises (1881 - 1973) 


\section{Contents}

1 Introduction $\quad 1$

1.1 Motivation ............................ 1

1.2 A hydrodynamic ansatz . . . . . . . . . . . . . . . 5

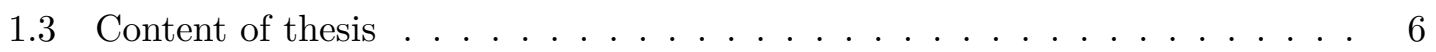

2 Kinetic theory of granular gases $\quad 9$

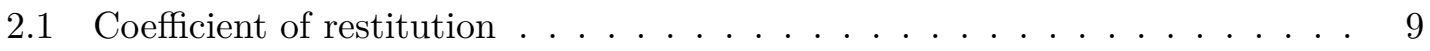

2.2 Coefficient of restitution in the viscoelastic model . . . . . . . . . . . . . . 12

2.3 Knudsen number . . . . . . . . . . . . . . . . . . . . . . . 14

2.4 Velocity distribution function . . . . . . . . . . . . . . . . 16

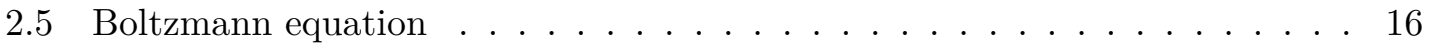

2.6 Hydrodynamic equations . . . . . . . . . . . . . . . . . . . . . . . . 19

2.7 Transport coefficients . . . . . . . . . . . . . . . . . . . 21

2.8 Haff's law for a free cooling granular gas in the homogeneous cooling state . 26

2.9 Linear stability analysis of the homogeneous cooling state . . . . . . . . 28

3 Navier-Stokes equations of granular gases 33

3.1 Pair correlation function . . . . . . . . . . . . . . . . . . . 33

3.2 Quasi conservative form of the Navier-Stokes equations . . . . . . . . . . . 35

3.3 Dimensionless form of the Navier-Stokes equations . . . . . . . . . . . . 36

3.4 Reynolds, Prandtl and Mach number in granular gases . . . . . . . . . . . . 40

4 Computational model $\quad 42$

4.1 Programming on graphics processing units . . . . . . . . . . . . . . . . 44

4.2 Overview of the numerical solver . . . . . . . . . . . . . . . 45

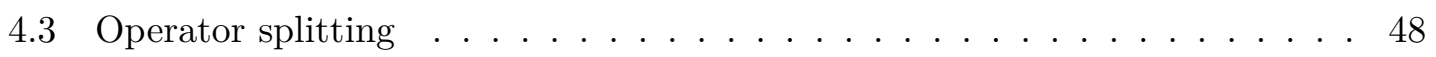

4.4 Finite-volume method . . . . . . . . . . . . . . . . . . . . . 49

4.5 Gauss-Legendre integration . . . . . . . . . . . . . . . 51

4.6 Polynomial reconstruction: WENO method . . . . . . . . . . . . . . . 54

4.7 WENO method for derivatives . . . . . . . . . . . . . . 57

4.8 Riemann problem . . . . . . . . . . . . . . . . . . 58

4.9 MUSCL-Hancock method . . . . . . . . . . . . . . . . . . 62

4.10 Centered fluxes . . . . . . . . . . . . . . . . . 63

4.11 MUSTA flux . . . . . . . . . . . . . . . . . . . 64 
4.12 ADER scheme . . . . . . . . . . . . . . . . . . . 65

4.13 Courant-Friedrich-Lewy condition . . . . . . . . . . . . 68

4.14 Diffusive flux . . . . . . . . . . . . . . . . . . . . 69

4.15 Boundary conditions . . . . . . . . . . . . . . . . . 72

4.16 Initialization . . . . . . . . . . . . . . . . . . . 74

5 A universal scaling law for the evolution of granular gases $\quad \mathbf{7 5}$

5.1 The global Mach number as an indicator for clustering . . . . . . . . . . . 75

5.2 Heuristic for the universal scaling . . . . . . . . . . . . . . . . 81

5.3 Correlation of density and temperature in the homogeneous cooling state . 82

5.4 Scaling issues . . . . . . . . . . . . . . . . . . . . 84

6 Shock waves as the origin of clustering in a granular gas $\quad \mathbf{8 8}$

6.1 Evolution of density, temperature and pressure fluctuations . . . . . . . 88

6.2 The role of subsonic convective waves . . . . . . . . . . . . . . 91

6.3 Comparison with molecular dynamical simulations . . . . . . . . . . . 94

6.4 Correlation between density, pressure and temperature in the homogeneous cooling state . . . . . . . . . . . . . . . . . . 97

6.5 Correlation between density, pressure and temperature in the linear stability

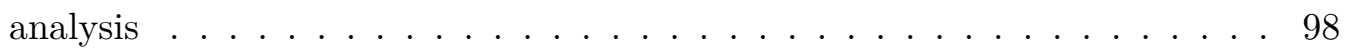

7 Declustering in a granular gas as a finite size effect 101

7.1 Long time behavior of granular gases . . . . . . . . . . . . . . . . 102

7.2 Clustering close to the critical system size . . . . . . . . . . . . . 103

7.3 Clustering in systems with solid walls . . . . . . . . . . . . . . 104

7.4 Cluster growth in the case of viscoelastic spheres . . . . . . . . . . 105

8 Extensions and Outlook $\quad 109$

8.1 Distributions of density, momentum and temperature . . . . . . . . . . . 109

8.2 The generation of a homogeneous, steady state in granular gases from an inhomogeneous state . . . . . . . . . . . . . . . . 112

8.3 Driven granular systems . . . . . . . . . . . . . . . . . . . . . . 114

8.4 Stochastic coefficient of restitution . . . . . . . . . . . 116

8.5 Central forces . . . . . . . . . . . . . . . . . . . . . . 119

9 Conclusions $\quad 122$

10 Appendix $\quad 135$

10.1 Eigenvalues of the linearized set of Navier-Stokes equation . . . . . . . . 135

10.2 WENO function in C-code . . . . . . . . . . . . . . . . . 136

10.3 Mathematica script for WENO seventh order . . . . . . . . . . . . 137 


\section{List of Figures}

1.1 Granular matter in our daily life. . . . . . . . . . . . . . . . . . 2

1.2 Sand in different collective states. . . . . . . . . . . . . . . . . . 3

1.3 Examples of granular gases: from terrestrial to cosmological scale. . . . . . 4

2.1 Collision cylinder of a particle. We assume that during a short $\Delta t$ the particle will collide with all particles, which have their centers of mass within the colliding cylinder of volume $\pi \sigma^{2} \bar{u} \Delta t \ldots \ldots \ldots \ldots$

2.2 Comparison of the full viscoelastic model (top panels) and the hybrid model (bottom panels). The figure shows the filling fraction (a), (d), the local Mach number $\mathcal{M}=\frac{v}{\sqrt{T}}(\mathrm{~b})$, (e) and the temperature (c), (f) in terms of the average temperature in the system. Both simulations started with the same random initial conditions and the snapshots are taken after a time $t=6000 \sigma / v_{t h, 0}$, where $v_{t h, 0}$ is the initial thermal velocity. The system parameters are: $L_{\text {sys }}=51200 \sigma, \bar{\phi}=0.01, E(t=0)=0.95$. The initial configuration is a homogeneous cooling state. . . . . . . . . . 25

2.3 Difference of the filling fraction between the full viscoelastic model (Fig. 2.2(a)) and the hybrid model (Fig. 2.2(b)) . . . . . . . . . . . 26

2.4 Real part of the eigenvalues of the reduced system in Eq. (2.87). The figure

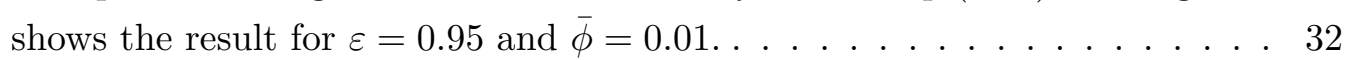

4.1 Flow chart of the structure of the algorithm for the direct numerical simulations. . . . . . . . . . . . . . . . . . . . 4 47

4.2 A schematic sketch of the multidimensional reconstruction method (a) and the dimension by dimension method (b) and (c) in two dimension. The reconstruction is performed from central points $\boldsymbol{U}$. The multidimensional method uses stencil points in all available dimensions to construct a polynomial from where the Gaussian stencil point can be calculated. In contrast the dimension by dimension reconstruction is performed in two sweeps. In the first sweep the black stencils are used to construct a one-dimensional polynomial from where the green intermediate points are calculated. During the second sweep we achieve from the green intermediate stencils the final Gaussian stencil point $\boldsymbol{U}_{\alpha, \beta, \gamma}$ along a second one-dimensional reconstruction. 52

4.3 Sketch of the Gaussian stencil points on the surface of a 3D finite volume. . 53 
4.4 Reconstruction on a 2D surface to obtain the stencils $g_{i}$. Panel (a) displays the reconstruction in 2 steps using an intermediate point (green circle). Panel (b) displays the reconstruction in one step. . . . . . . . . . . . . . . 53

4.5 The problem of reconstruction of the value $\boldsymbol{U}_{i+\alpha}$ at $x=x_{\alpha}$ as an example of $r=3$. The left panel shows the small construction polynomials $p^{1}, p^{2}, p^{3}$ and the right panel shows the large construction polynomial $P$. The figure shows the problem for a FDM for the sake of simplicity; in the FVM the exact points have to replaced with the averages over the corresponding cell. 55

4.6 Riemann problem: $U^{L}$ and the resulting flux $f^{L}$ is the initial solution $(t=0)$ to the left of $x_{i+\alpha}, U^{R}$ and the corresponding flux $f^{R}$ is initial solution to the right of $x_{i+\alpha}$. The problem is to determine the initial flux $f^{M}$ at position

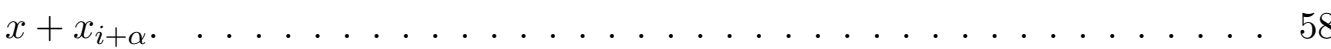

4.7 Characteristics of the linear advection equation $\partial_{t} U+a \partial_{x} U=0$ with $a>0$ and a discontinuity at $x=0$, different characteristic correspond to different constants. On each characteristic the value $U$ is constant. . . . . . . . . . . 59

4.8 Picture of a Riemann fan. The characteristics shown distinguish the different states from each other.

4.9 Numerical errors which appear during approximation. Panel (a) displays the effect of numerical dissipation and panel (b) displays the oscillations caused by numerical dispersion . . . . . . . . . . . . . . . . . 64

4.10 Wave propagation through finite volumes. In the time step $\Delta t$ the orange wave propagates by $\delta=\frac{\Delta t}{\omega_{\max }}$. Panel (a) shows the unphysical case when the wave propagation skips the volume $i$ during one time step. Panel (b) shows the necessary condition for stability, the propagation length $\delta$ has to be below $\Delta x \ldots \ldots \ldots \ldots \ldots \ldots \ldots$

4.11 A sketch of a 2D system with two ghost cells at each boundary location. The values of the ghost cells must be set to satisfy the needed boundary conditions. . . . . . . . . . . . . . . . . . . . 73

5.1 Snapshots of the system showing the temporal evolution of a three-dimensional granular gas with a coefficient of restitution $\varepsilon=0.9$ and average filling fraction $\bar{\phi}=0.05$. (Top row) From left to right, snapshots of the density field at times $t=10^{9}, t=10^{10}$ and $t=10^{11}$, respectively. The gray scale represents the local average density. (Bottom row) Three-dimensional map of the temperature field $T(\boldsymbol{r}, t)$ at the same times of the corresponding density plot in the top row. The color (red - hot; blue - cold) represents the local temperature compared to the average temperature in the system. . . . . . 76 
5.2 Temporal evolution of the maximum filling fraction $\phi_{\max }(\mathrm{a})$ and of the filling fraction fluctuations $\left\langle(\delta \phi)^{2}\right\rangle$ (b). In (a) and in the main panel of (b) the average filling fraction $\bar{\phi}=0.05$. The time is measured in units of $\sigma$ over the initial thermal velocity $v_{t h, 0}$. The clustering process exhibits the same qualitative features over a wide range of coefficients of restitution $\varepsilon$ and average filling fractions $\bar{\phi}$. The curves are guides to the eye. As $\varepsilon$ grows, the time of onset of clustering increases of four orders of magnitude. The inset panel shows the effect of varying average filling fractions. The initial crossover at $\left\langle(\delta \phi)^{2}\right\rangle \approx 10^{-16}$ is an effect of the initial transient. . . . . . . . 77

5.3 (a) Temporal evolution of density fluctuations for different initial values of the Mach number $\mathcal{M}_{0}$. (b) Evolution of the kinetic energy (symbols) for the same initial Mach numbers as in (a), and evolution of the temperature (solid, magenta line). We mark the times $t^{*}\left(\mathcal{M}_{0}\right)$ when the kinetic energy equals the temperature and the corresponding values of $\left\langle(\delta \phi)^{2}\right\rangle\left(t^{*}\right)$ (vertical, dotted lines). To test the dependence of the density fluctuations on $\mathcal{M}$, we vary the initial, inertial velocities in the system, that is, the initial value of the Mach number $\mathcal{M}_{0}$. We find that for larger $\mathcal{M}_{0}$ the onset of clustering occurs decades in time earlier, but still it coincides with the time when $\mathcal{M} \sim \mathcal{O}(1) \ldots \ldots \ldots \ldots \ldots \ldots \ldots \ldots$

5.4 The density fluctuations exhibit a universal scaling in terms of the system's average Mach number $\mathcal{M}$. After the relaxation of the initial conditions the granular gas shows a collapse of the density fluctuations on the curve $\left\langle\delta \rho^{2}\right\rangle(\mathcal{M})=c \mathcal{M}^{2}$. Calculations are shown for system at $\bar{\phi}=0.05, \varepsilon=0.9$ and $\mathcal{M}_{0}=10^{-8}$ unless the variable are explicitly changed according to the legend. . . . . . . . . . . . . . . . . 79

5.5 Free cooling systems with different initial fluctuation in the density and temperature. The system parameters are $\bar{\phi}=0.05, \varepsilon=0.9$ and $\mathcal{M}_{0}=$ $10^{-8}$. The figure displays that after a certain time the initial conditions are smoothed out. . . . . . . . . . . . . . . . . 80

5.6 Density fluctuation for a model of viscoelastic spheres and const $\varepsilon$ in 3D. Both models follow the same scaling behavior. . . . . . . . . . . . . . 80

5.7 Panel (a) shows the temperature and density at different times in the HCS, panel (b) shows their correlation in the last figure of panel (a). Panel (c) plots the temporal evolution of the correlation coefficient $\operatorname{Cor}(\rho, T)$ during the entire HCS (the transition to the ICS appears in the region of $t=10^{10}$ ). (The data are the same as in Fig. 5.1.) . . . . . . . . . . . . . 83

5.8 Density field of a freely cooling system at different system sizes. (a) $L_{\text {sys }}=$ $5120 \sigma$, (b) $L_{s y s}=256000 \sigma$, (c) $L_{s y s}=512000 \sigma \ldots \ldots \ldots$. . . . . 84

5.9 Freely cooling gas after transition to an ICS; $\varepsilon$ is constant and $\bar{\phi}=0.01$. The panels show snapshots for (a) $\varepsilon=0.5, L_{\text {sys }}=10240 \sigma$, (b) $\varepsilon=0.9, L_{\text {sys }}=$ $25600 \sigma,(\mathrm{c}) \varepsilon=0.99, L_{\text {sys }}=102400 \sigma$, (d) $\varepsilon=0.9999, L_{\text {sys }}=2048000 \sigma \ldots 85$ 
5.10 Characteristic cluster sizes in cooling granular gases after the transition to in ICS. . . . . . . . . . . . . . . . . . . . . . 86

5.11 Cooling granular gas in the ICS with $\bar{\phi}=0.01, \varepsilon=0.9$. The initial convective velocities are of $\mathcal{O}\left(10^{-8}\right)$ in panel (a) and $\mathcal{O}\left(10^{-2}\right)$ in panel (b). There is no qualitative nor quantitative difference in the characteristic of the visible clusters. . . . . . . . . . . . . . . . . . 86

6.1 (a) Fieldmaps of the local Mach number and (b) local density at time $t \approx 10^{6}$. The system has periodic boundary conditions; the initial condition in Eq. (6.1) are used, the average filling fraction $\bar{\phi}=0.005$ and $\varepsilon=\varepsilon(T), \varepsilon(0)=0.9$. (c) Evolution of the pressure, density, temperature and the maximum Mach number in the center of 2D system. Apart from the local Mach number, the quantities are normalized and the deviation from the average value is plotted. . . . . . . . . . . . . 90

6.2 Final state of a freely cooling granular gas with. Fieldmaps of the local density (a), local pressure (b) and the local Mach number (c). The system has periodic boundary conditions; the initial condition are similar to Eq. (6.1), but the initial center density is $\rho=1.1, r<R$, the average filling fraction $\bar{\phi}=0.05$ and $\varepsilon=\varepsilon(T), \varepsilon(0)=0.9 \ldots \ldots \ldots \ldots$

6.3 Evolution of a free cooling granular gas which was initialized with a subsonic convective wave. The initial subsonic wave has an amplitude of $10^{-6} v_{t h, 0}$. The panels (a),(d),(g) display the field maps of the density, panels (b), (e),(h) display the pressure in terms of the average pressure and the panels (c),(f),(i) display the local Mach number in the system. The subsonic wave in the initial state (panel (a)-(c)) turns to supersonic speed (panel (d)-(f)) and reaches eventually high Mach numbers (panel (g)-(i)). We can locate the transition from HCS to ICS at the time when the wave turns supersonic (panel $(d)$ ). The system has periodic boundary condition in $x$ direction and hard walls with no-slip boundary conditions in $y$-direction; the average filling fraction $\bar{\phi}=0.005$ and $\varepsilon=\varepsilon(T), \varepsilon(0)=0.9 \ldots \ldots 92$ 
6.4 Evolution of a freely cooling granular gas which was initialized with a subsonic convective wave in 3D. The initial subsonic wave has an amplitude of $10^{-4} v_{t h, 0}$. The panels (a),(c) display cross sections of the fieldmap of the local Mach number and panels (b),(d) display cross sections of the field map of the density in the system. The subsonic wave in the initial state turns to supersonic speed (panel (a)) and reaches eventually high Mach numbers (panel (c)). We can locate the transition from HCS to ICS at the time when the wave turns supersonic (panel (b)). The system has periodic boundary condition in all directions; the average filling fraction $\bar{\phi}=0.01$ and $\varepsilon=\varepsilon(T), \quad \varepsilon(0)=0.9$. The system is not as stable as the $2 \mathrm{D}$ simulation in Fig. 6.3, therefore the final state(panel (c),(d)) is not as far developed in time as it is in Fig. 6.3(g)-(i). . . . . . . . . . . . . . . . 93

6.5 A granular gas in the ICS. (a) field map of the filling fraction. (b) pressure in the system. The high correlation between pressure and density is obvious. (c) field map of the local Mach number. The cutoff is set at $\mathcal{M}=1$ to distinguish between sub- and supersonic waves. The system has periodic boundary conditions, the average filling fraction $\bar{\phi}=0.01$ and $\varepsilon_{\text {const }}=0.9$. 94

6.6 MD simulations of two freely cooling systems. (a), (b), (c) show the evolution of a system with $L_{\text {sys }}=8000 \sigma, 2.560 .000$ particles and $\varepsilon=0.9$. (d), (e), (f) show the evolution of a system with $L_{\text {sys }}=1000 \sigma, 40.000$ particles and $\varepsilon=0.9$. Both simulations start from a homogeneous state with a well defined initial convective flow as in the DNS in Fig. 6.3. The initial convective flux is $10 \%$ of the thermal velocity in the top panels and $45 \%$ in the bottom ones. The critical system size for both configurations is about $360 \sigma .95$

6.7 Dependency of the onset time of clustering on the initial strength of a convective wave. The results are taken from MD simulation with $N=$ 40.000, $N=640.000$ particles and from DNS. In all simulation the filling fraction is $\bar{\phi}=3.14 \%$ and $\varepsilon_{\text {const }}=0.9$. Results for MD simulation are obtained from an average over $2-5$ simulations with different initial random particle locations and velocities. The lines are a guide to the eye. . . . . . 96

6.8 Correlation between density and pressure for free cooling granular gas. (a) displays some DNS calculations as in Fig. 6.5 (2D system). (b) displays data from MD simulation with $\varepsilon=0.7$ and 160.000 particles (2D system). (c) displays DNS results of a free cooling 3D system with $\varepsilon=0.9$ and $\bar{\phi}=3.14 \%$. The green line marks the time around which the transition to ICS appears. . . . . . . . . . . . . . . . . . . . 97 97

6.9 Asymptotic normalized perturbations for two different initial perturbations. A correlation between $\delta$ and $\pi_{p}$ can be observed. The temperature perturbation is anti-correlated to $\delta$ and $\pi_{p} \ldots \ldots \ldots \ldots \ldots \ldots \ldots \ldots \ldots \ldots \ldots$ 
7.1 Temporal evolution of the average kinetic energy $E_{k i n}$ and the average granular temperature $T$ for a granular gas with average filling fraction $\bar{\phi}=0.05$. (a) Granular system with fixed coefficient of restitution $\varepsilon=0.99$ and $L=1920$. (b) Granular system with variable coefficient of restitution and initial value $\varepsilon_{0}=\varepsilon(t=0)=0.99$ and $L=5120$. The clustering appears at the crossover of thermal energy and kinetic energy of convective velocities. In both cases thermal and kinetic energy couple at the transition point and undergo a Haff's cooling afterwards. In the case of viscoelastic particles, we can observe a decoupling of thermal and kinetic energy, which sets the onset point of declustering. . . . . . . . . . . . . . . 102

7.2 Asymptotic value of the fluctuations in the ICS as function of relative system size to the critical size $\mathfrak{l} \equiv L / L_{\text {crit }}$. For a fixed coefficient of restitution the fluctuations reach values which depend on the system size. The plot shows simulations for $\varepsilon=0.9$ and $\varepsilon=0.999$ and $\bar{\phi}=0.05$. The inset shows the same data on a linear scale. . . . . . . . . . . . . . . . . 104

7.3 A freely cooling granular gas inside a box with rough hard wall. The friction coefficient on the wall is $C_{f}=0.9$ (compare initial condition in section 4.16). The system size is $1280 \sigma \times 1280 \sigma, \bar{\phi}=0.1$ and $\varepsilon=0.99$. We observe clustering along the walls only for systems larger than the critical system size. The system is about 4 times larger than $L_{\text {crit }}$. . . . . . . . . . . . 105

7.4 Temporal evolution of density fluctuations in 2D granular gases with an average filling fraction $\bar{\phi}=0.05$. The black circles correspond to calculations at constant $\varepsilon=0.99$. All other symbols correspond to calculations for viscoelastic particles where $\varepsilon_{0}=\varepsilon(t=0)=0.99$. The critical system size for this $\varepsilon_{0}$ is $L_{\text {crit }} \approx 500$. If the system is large enough, the fluctuations grow till the clustering becomes visible. For a constant $\varepsilon$ the fluctuations reach a plateau. When $\varepsilon=\varepsilon(T)$ the fluctuations first grow to a size dependent maximum but eventually vanish after a certain time. This time grows with system size . . . . . . . . . . . . . . . . . . . . 106

7.5 The time $t^{*}$ characterizing the disappearance of clusters grows with the size of the system in 2D (panel (a)) and in 3D (panel (b)). Results from DNS calculations are shown as circles. The solid line represents a power law with exponent 12. The value of $t^{*}$ for the smallest $L$ does not fall on the power law because the ICS is not fully developed (compare with Fig. 7.4). . . . . 106

7.6 Evolution of a freely cooling granular gas with viscoelastic spheres at time $t=3.7 \cdot 10^{8}(a), t=1.3 \cdot 10^{9}(b), t=6.3 \cdot 10^{9}(c), t=1.4 \cdot 10^{11}(d)(t$ in units of $\left.\left[\frac{\sigma}{v_{t h, 0}}\right]\right)$. The cluster form and growth till they reach system size (see the full movie attached under viscoelasticCooling.avi) . . . . . . . . . . . . 107 
7.7 Typical length of the clusters during the inhomogeneous cooling process for $\varepsilon(t=0)=0.99$ and $L=5120$ and $L=10240$. The values fluctuate and grow till values above 0.5. The time at which this value is reached corresponds to the disappearing of clusters in the system (see Fig. 7.4). We calculate the length scale $\lambda$ from the first moment of the Fourier transform of the density correlation function. . . . . . . . . . . . . 108

8.1 Distribution of the momentum (a), density and temperature (b) in the HCS. The system has a size of $(25600 \sigma)^{2}, \bar{\phi}=0.01$ and $\varepsilon=0.9$ and is divided into $1024^{2}$ finite volumes. The distribution is calculated at a temperature $\langle T\rangle \approx 10^{-12}(T(t=0)=1)$; the convective velocities are 4 orders of magnitude below the thermal velocities. . . . . . . . . . . . . . . 110

8.2 (a) Snapshot of the system from which various probability distributions were calculated. Distribution of the momentum (b), density (c) and temperature (d) in the ICS. The system has a size of $(25600 \sigma)^{2}, \bar{\phi}=0.01$ and $\varepsilon=0.9$. The power law fit in (c) is $y(x)=0.0439 x^{-3.52}$.

8.3 Creation of a homogeneous granular gas via shaking of the system in the $x$ - and $y$ - direction. Two different initial configurations are shown. The frequency is $f=20 \mathrm{~Hz}$, the ampliutude $A=0.14 \sigma$ and the system size, $L=285 \sigma$. The pictures are taken after $0,140,760,2940,6940$ cycles ((a) (e)) and after $0,160,300,1280,7340$ cycles $((\mathrm{f})-(\mathrm{j})) \ldots \ldots 113$

8.4 Steady state of driven granular media. The systems are driven in the vertical direction with a frequency of $50 \mathrm{~Hz}$ and a strength of $\Gamma=12 \mathrm{~g}$. In addition there is constantly $1 g$ gravity force acting on the system in the downward direction. The systems contain hard wall boundary conditions on each side with a friction strength of $C_{f}=0.9$ (see Section 4.16 for details on boundary conditions). The system size is $256 \sigma \times 256 \sigma$ in (a), $461 \sigma \times 115 \sigma$ in (b) and $630 \sigma \times 115 \sigma$ in (c). (d)-(f) are results from experiments; the pictures are taken from Eshuis et al. (2007) [1]. . . . . . . . . . . . . . . . . 115

8.5 $\bar{\phi}$ - $F$-phase diagram of driven granular gases. We highlight some representative states with the field maps of the density. The normalized force $\Gamma$ is measured in terms of the magnitude of the external force. The color map for the convection rolls displays the vorticity field of the granular gas. Red (blue) color indicates positive (negative) vorticity. . . . . . . . . . . . 115

8.6 Example data for a stochastic variable which follows a uniform distribution (a), a normal distribution (b) and a Laplace distribution (c). . . . . . . 117

8.7 Shape of dense clusters after the transition from HCS to ICS. The filling fraction is $\bar{\phi}=0.05$. (a) Results for a system whit constant $\varepsilon_{M}=$ $0.9, \sigma_{\varepsilon}=0$. (b) Results for a system with a distribution of $\varepsilon$ with the

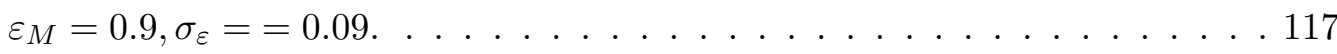


8.8 Dependency of the typical cluster size on $\sigma_{\varepsilon}$ for different distributions after the transition from HCS to ICS; (a) uniform distribution, (b) normal distribution, (c) exponential distribution. . . . . . . . . . . . . 118

8.9 Temperature evolution for system with different $\sigma_{\varepsilon}$ in the normal distribution; $\varepsilon_{M}=0.9, \bar{\phi}=0.05$. The orange line marks Haff's law. . . . . . . . . 119

8.10 Evolution of a 2D granular gas under the effect of gravity. The center of the gravitational interaction is placed in the center of the system. The system was initialized with a small positive velocity of $10^{-4} v_{t h, 0}$ in the angular direction. The system has $\varepsilon=0.9, \phi=0.01$; in the center $\phi=0.0001$ to avoid a density collapse. Open boundary condition in $x$ - and $y$-direction are used. . . . . . . . . . . . . . . . . . . . . 120 


\section{List of Tables}

2.1 Coefficients for full viscoelastic model and the Taylor expansion of the static transport coefficients. . . . . . . . . . . . . . . . . . . 24 


\section{List of movies}

\begin{tabular}{|c|c|}
\hline 3Dcooling.avi & freely cooling granular gas in $3 \mathrm{D}$ \\
\hline centralFluct.avi & $\begin{array}{l}\text { freely cooling granular gas in } 2 \mathrm{D} \\
\text { with a small density offset in the center }\end{array}$ \\
\hline centralFluctViscoelast.avi & $\begin{array}{l}\text { freely cooling granular gas in } 2 \mathrm{D} \text { with a small } \\
\text { density offset in the center (viscoelastic spheres model) }\end{array}$ \\
\hline coolingInBoxNoClustering.avi & $\begin{array}{l}\text { freely cooling granular gas in a } \\
\text { container with friction at the walls, no clustering }\end{array}$ \\
\hline coolingInBoxClustering.avi & $\begin{array}{l}\text { freely cooling granular gas in a container with } \\
\text { friction at the walls, with clustering at the walls }\end{array}$ \\
\hline subsonicInitialWave.avi & $\begin{array}{l}\text { freely cooling granular gas initialized } \\
\text { with a small subsonic wave (DNS simulation) }\end{array}$ \\
\hline CurvedSeededShock.avi & $\begin{array}{l}\text { freely cooling granular gas initialized } \\
\text { with a small subsonic wave (MD simulation) }\end{array}$ \\
\hline freeCoollingCloseCritSize.avi & $\begin{array}{l}\text { freely cooling granular gas close above } \\
\text { the critical system size }\end{array}$ \\
\hline viscoelasticCooling.avi & $\begin{array}{l}\text { freely cooling granular gas of } \\
\text { viscoelastic spheres model in } 2 \mathrm{D}\end{array}$ \\
\hline convectionRolls.avi & $\begin{array}{l}\text { vibrated granular gas showing } \\
\text { convection rolls and Leidenfrost effect }\end{array}$ \\
\hline convectionRollsVorticity.avi & $\begin{array}{l}\text { vibrated granular gas showing convection rolls } \\
\text { and Leidenfrost effect (movie of the vorticity) }\end{array}$ \\
\hline granularLeidenfrost.avi & $\begin{array}{l}\text { vibrated granular gas showing the } \\
\text { granular Leidenfrost effect }\end{array}$ \\
\hline centralForce.avi & $\begin{array}{l}\text { granular gas evolving under the present of } \\
\text { an attractive central force }\end{array}$ \\
\hline MpiLogo.avi & $\begin{array}{l}\text { freely cooling granular gas with initialized } \\
\text { subsonic convective waves }\end{array}$ \\
\hline
\end{tabular}




\section{Symbols}

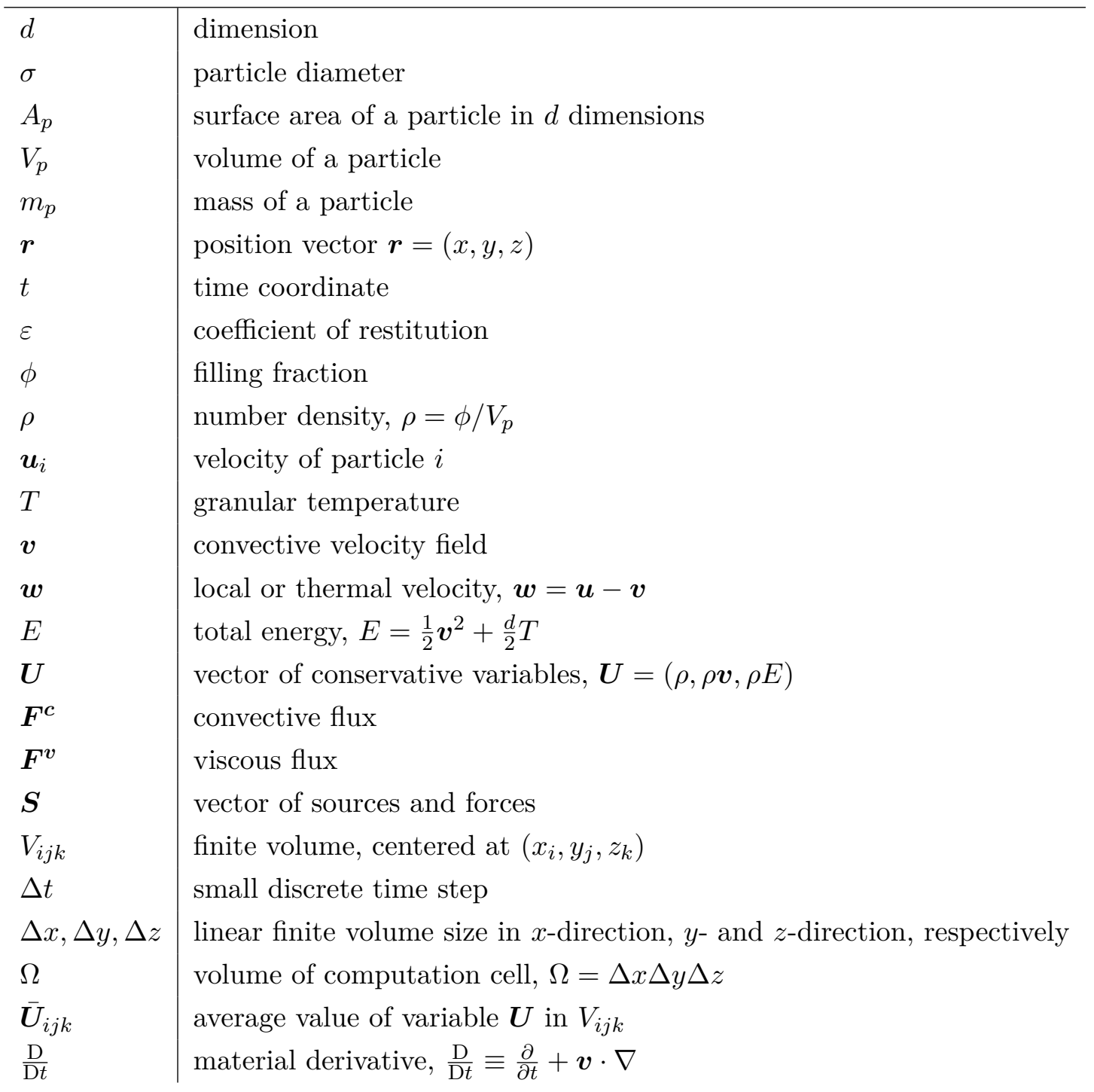

Throughout the thesis we use different fonts to distinguish a scalar $a$, from a vector $\boldsymbol{a}$, from a matrix or tensor $\underline{\mathbf{A}}$. 


\section{Acronyms}

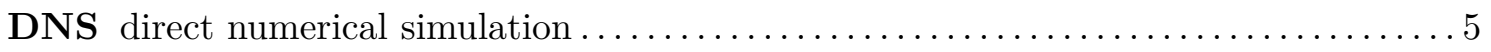

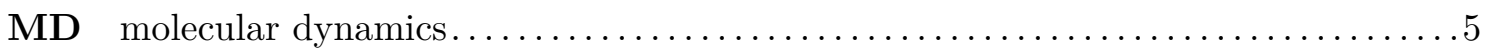

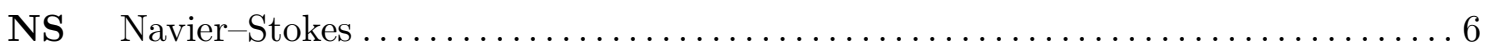

dGRP diffusive generalized Riemann problem $\ldots \ldots \ldots \ldots \ldots \ldots \ldots \ldots \ldots \ldots \ldots \ldots$

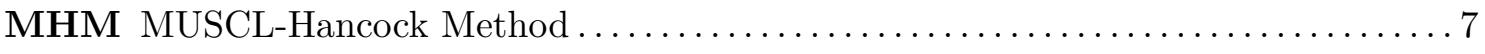

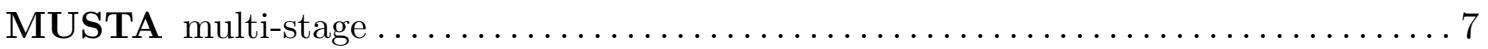

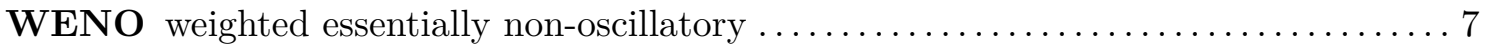

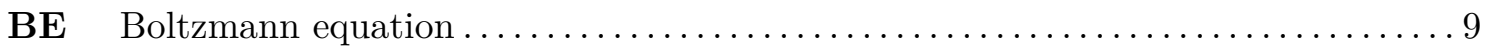

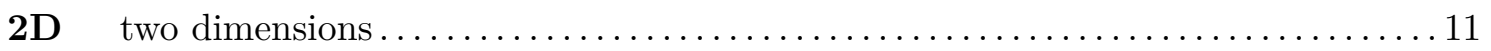

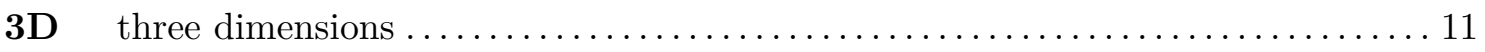

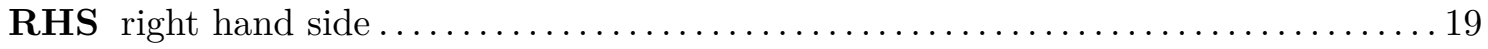

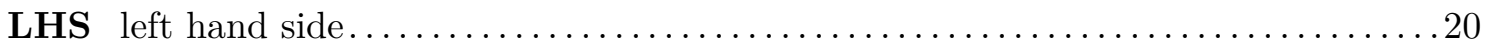

HCS homogeneous cooling state.................................. 28

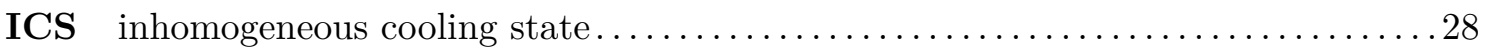

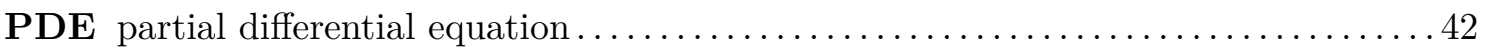

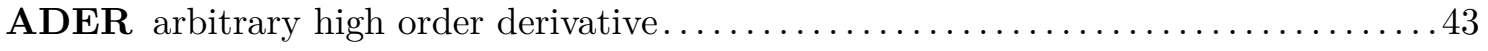

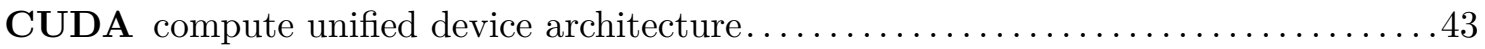

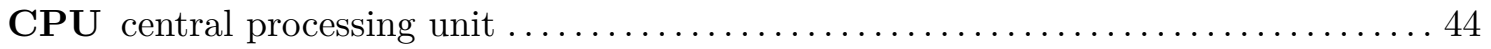

GPU graphics processing unit................................... 44

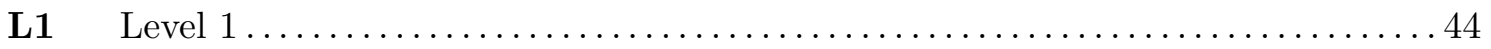

NVCC Nvidia CUDA compiler .................................... 44

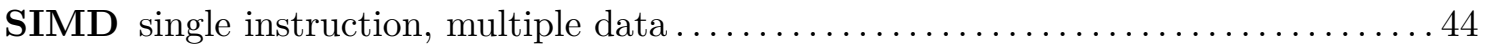




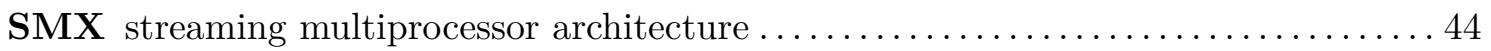

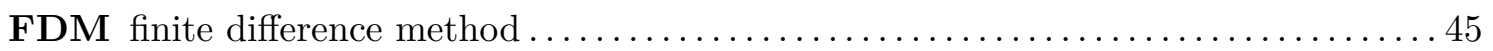

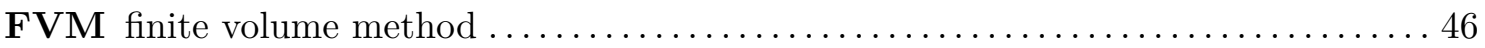

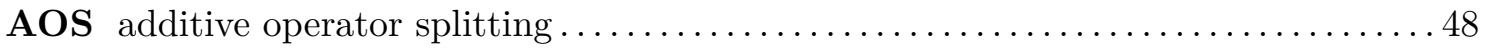

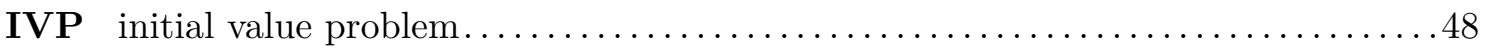

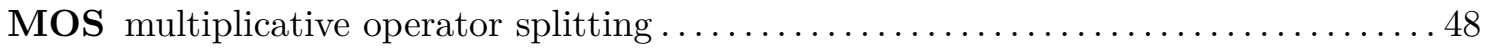

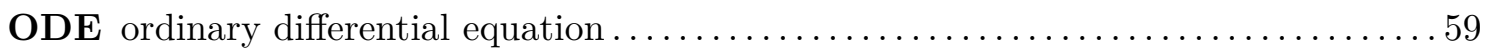

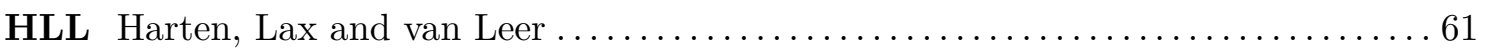

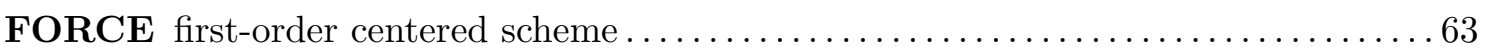

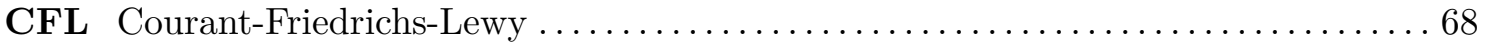




\section{Introduction}

\subsection{Motivation}

Among all the fascinating events taking place around us, the granularity of materials is extraordinarily intriguing. It is still unclear how dense, terrestrial planets can form out of the nebular dust and particles. This question is of fundamental importance for astrophysical problems, but also for our current understanding of life and its emergence. As a young star cools down to the T Tauri stage, molecular material coalesce into micronsize dust grains mainly composed of iron, nickel, aluminum and silicates. These grains grow to kilometer-size objects via mechanisms still largely unknown, however this is the length scale where granular physics is relevant. Therefore, granular matter probably plays an important role in the formation of planets. A deeper understanding of the fundamental processes of granular dynamics may also give a lot of insight at the frontiers of other fields of modern physics: Plinian eruptions are connected to an enormous amount of ashes which contains granular particles, avalanches are composed of granular ice particles. We find grains in all kinds of suspensions and even some biological multicellular systems could be understood within a granular framework. For example, nematic ordering in growing colony of non-motile bacteria, or the patterns of asters and vortices formed by the microtubule in the cytoskeleton (see [2] for more details). Also in our daily life we find granular media everywhere: cereals in agriculture, sand at the beach or rice at dinner are only a few examples. Beside our daily experience, granular materials are highly important in industry: a lot of raw materials, especially in the plastic industry, are granular materials. In Fig. 1.1 we see a few examples of common granular materials.

Granular materials are collections of grains larger than a micron for which molecular or Brownian fluctuations are irrelevant. Thus, every single grain is a macroscopic object. The scale where we can find granular materials is enormous: from small dust particles up to whole galaxies, we can describe matter in the context of grains.

Granular materials may not just be of uniform composition but may result of all kinds of 

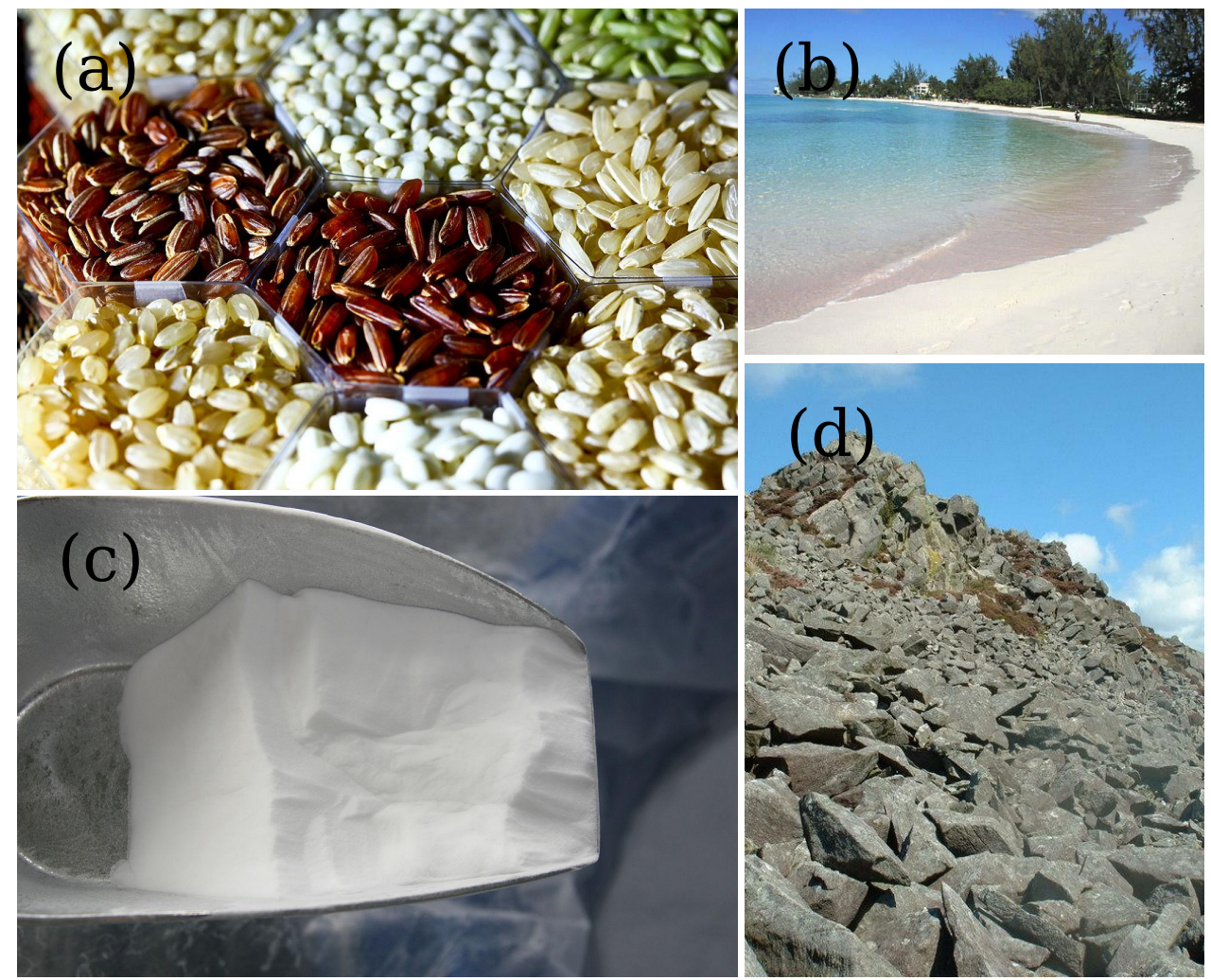

Figure 1.1: Granular matter in our daily life. (a) Different kinds of rice ${ }^{1}$. (b) A beach in Barbados $^{2}$. (c) Silicate in industry ${ }^{3}$. (d) Rubble at the British northern sea coast ${ }^{4}$.

elements, chemical combinations or mixtures. However, even if the microscopic description of a granular material might be extremely complex, only a few parameters are necessary to describe the macroscopic state. The defining characteristic of granular matter is the dissipative nature of its interactions. In granular collisions, the component of the relative velocity of the colliding particles along the line joining the two centers of mass decreases in absolute value by a factor $0<\varepsilon<1$, called coefficient of restitution. The reason for the dissipative nature are inner degrees of freedom inside a granular particle and inelastic deformations. If grains would not act dissipative they would behave as a macroscopic equivalent of atoms. Indeed we find numerous similarities to classical states of matter as we can find granular material in a solid-like, liquid-like and gas-like state.

As shown in Fig. 1.2, it is fascinating to observe that granular materials can form a solid-like sand castle, but sand is also able to flow like a liquid inside an hourglass, and finally we can even witness a gas-like behavior when we watch a sand storm or a snow avalanche.

The dissipative nature of grains leads to phenomena which are not known in nondissipative systems. In granular system we observe configurations of particles rendered

\footnotetext{
${ }^{1}$ By IRRI Images, CC BY 2.0, https://commons.wikimedia.org/w/index.php?curid=11202403

${ }^{2}$ User: Ranveig, CC BY 2.0, https://commons.wikimedia.org/w/index.php?curid=323926

${ }^{3}$ Robin Müller, CC BY-SA 3.0, https://commons.wikimedia.org/w/index.php?curid=16264199

${ }^{4}$ Brian John, CC BY-SA 2.0, https://commons.wikimedia.org/w/index.php?curid=12905860
} 

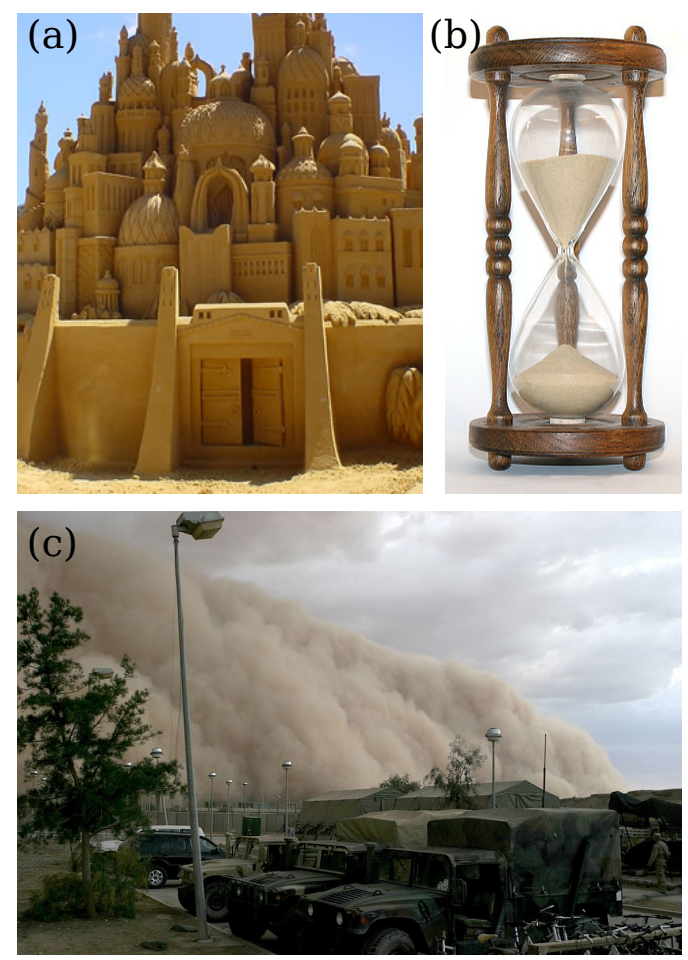

Figure 1.2: Sand in different states. (a) An elaborate sand castle ${ }^{5}$. (b) Hourglass ${ }^{6}$. (c) Sandstorm in Al Asad, Irak ${ }^{7}$.

stable or metastable because of dissipation. In general, a granular system does not evolve towards free-energy minima, because there is no free energy, nor any obvious minimization principle. The jamming transition is a well known example and a pretty annoying problem in agriculture. The problem that arises here is that a granular flow stops because the grains stuck together and build a dense cluster, historically we find this problem in silos and when seeding a field. Another example of transition can be found in snow avalanches. When the avalanche flows a person sinks into the snow because it behaves like a liquid, but once the snow comes to rest one is confined in a dense, cold prison. Yet another example for non-Newtonian fluids where granular media are present are quicksands; quicksand is solid like but it turns to liquid under very small stresses.

In this thesis we mainly study a nonequilibrium transition which occurs in clouds of granular particles, namely in granular gases without external forces. A granular gas cools down over time just due to the dissipative collisions between particles. Cooling means in this context that the kinetic energy of the system drops down and the particles become much slower over time. At variance with an ordinary gas, such a cooling granular gas will suddenly form dense, filamentary clusters. Eventually we do not find a granular gas anymore but dense solid regions. We will show in this thesis when the clustering in a freely cooling granular gas appears and we will describe the driving process behind it. We

\footnotetext{
${ }^{5}$ Public Domain, https://commons.wikimedia.org/w/index.php?curid=67485

${ }^{6}$ User:S Sepp, CC BY-SA 3.0, https://commons.wikimedia.org/w/index.php?curid=2949887

${ }^{7}$ Corporal Alicia M. Garcia, U.S. Marine Corps. [Public domain], via Wikimedia Commons
} 
discuss the similarities and differences for systems of constant and variable coefficient of restitution.
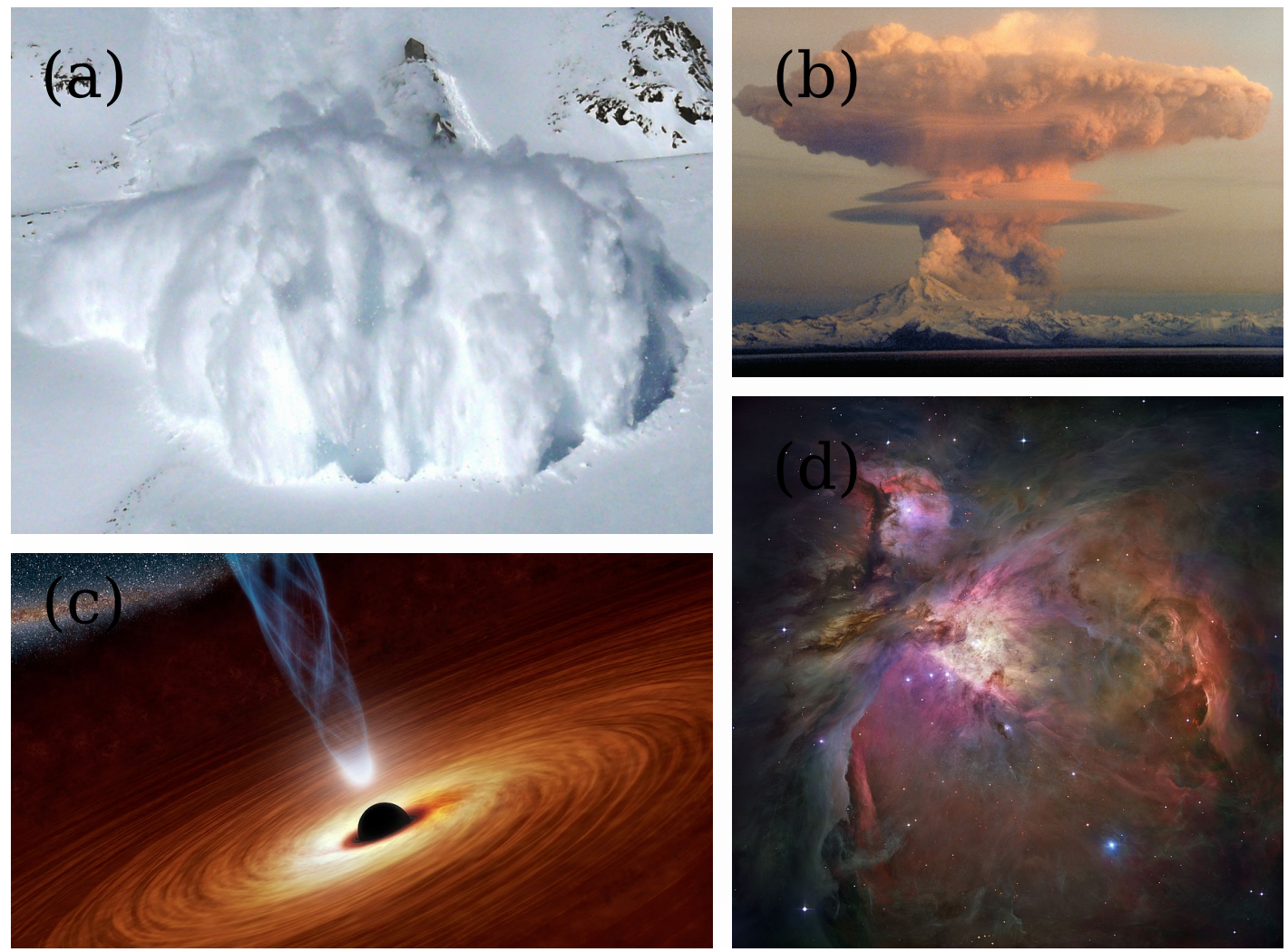

Figure 1.3: Examples of granular gases: from terrestrial to cosmological scale. (a) A dust avalanche $^{8}$. (b) Ascending eruption cloud from Redoubt Volcano as viewed to the west from the Kenai Peninsula ${ }^{9}$. (c) Accretion disc of a black hole, NASA artist's concept ${ }^{10}$. (d) An unprecedented look at the Orion Nebula by the Hubble Space Telescope captured ${ }^{11}$.

If a granular system consists of particles which can move freely because they have a large mean free path, we call such a state the gas state of a granular material. Within this thesis we concentrate on the physics of granular gases. Granular gases have been used as model systems to describe geophysical processes such as Plinian eruptions [3, 4], the solar corona [5], the asteroid belt between Mars and Jupiter, planetary rings [6, 7], protoplanetary disks [8], and the formation of cosmological structures [9] because granular dissipative processes may play a role. Figure 1.3 shows example of the wide range of interesting phenomena where granular gas physics is present. Experimental studies [10-14] of granular gases are, however, rather scarce because of formidable challenges in preparing a system free of external forces. Microgravity experiments on parabolic flights or drop towers potentially offer good conditions [15], but it is very difficult to remove the influence of confining potentials or surrounding walls on the granular system.

\footnotetext{
${ }^{8}$ User: Scientif38, CC BY-SA 3.0, https://commons.wikimedia.org/wiki/File:2007-02-15-CLB-Couloir21c.JPG

${ }^{9}$ U.S. Geological Survey, http://pubs.usgs.gov/dds/dds-39/album.html

${ }^{10}$ [Public domain],http://www.nasa.gov/mission_pages/nustar/multimedia/pia16695.html

${ }^{11}$ [Public domain], https://www.spacetelescope.org/news/heic0601/
} 


\subsection{A hydrodynamic ansatz}

Not many experimental studies have been performed on granular gases. This has different reasons: microgravity experiments are typically quite expensive and hard to set up. But granular experiments are typically limited in size. A lot of interesting phenomena where granular gases might be important take place on astronomical scales which are not accessible by experiments.

If experiments are not available, computer simulations become even more important. In the past, numerous studies using molecular dynamics (MD) simulations have been performed. Because it is necessary to track every particle and to calculate the interactions between particles in MD simulations, it costs a lot of calculation time to simulate large systems. If we assume to have enough particles for a hydrodynamic limit, we can describe granular systems within a hydrodynamic theory. Then we can describe the dynamic by the evolution of smooth fields. Simulations which solve the field equations are called direct numerical simulation (DNS). The big advantage is the independence from the single particle scale, therefore it is typically not problematic to scale up the system within the DNS. Sadly this comes to the cost of a considerable effort to develop a stable and accurate algorithm or solver, but we will demonstrate in this thesis that we can obtain enlightening results out of it.

Hydrodynamic fields can be rigorously defined in a manner similar to molecular fluids by means of a coarse-graining of the microscopic kinetic equations. First, one considers the one-particle distribution function $f(\boldsymbol{r}, \boldsymbol{u}, t)$, which obeys the Boltzmann equation and represents the number of particles within a volume $\mathrm{d} \boldsymbol{r}$ centered at position $\boldsymbol{r}$ and with velocity $\boldsymbol{u}$ within the interval $\mathrm{d} \boldsymbol{u}$. Then, the transport equations for inelastic systems are derived by using a Chapman-Enskog expansion [16-18]. From the moments of $f(\boldsymbol{r}, \boldsymbol{u}, t)$, the number density $\rho(\boldsymbol{r}, t)$, convective velocity $\boldsymbol{v}(\boldsymbol{r}, t)$, and temperature $T(\boldsymbol{r}, t)$ fields can be derived. Physically, the granular temperature $T(\boldsymbol{r}, t)$ represents the fluctuations of the microscopic velocities $\boldsymbol{u}$ or, in other words, it is the energy of the uncoordinated motion of the particles.

In contrast to ordinary gases, the average kinetic energy of the system (granular temperature) steadily decreases because of the dissipative nature of a granular system, in the absence of convective fluxes. This temperature decrease was first studied by Haff [19] who gave an analytical expression for the dynamics of the temperature, nowadays known as Haff's law ${ }^{12}$

$$
T(t)=T_{0}\left(1+\frac{t}{\tau}\right)^{-\alpha}
$$

where $T_{0}$ is the initial temperature, $t$ is the time, $\tau$ the characteristic cooling time [20], and $\alpha=2$ if the coefficient of restitution $\varepsilon=$ const. This cooling process is well understood in theory [19], simulations [21-25] and experiments [10-12, 14, 26].

\footnotetext{
${ }^{12}$ The law is given nowadays for the temperature but indeed Haff derived the dynamics of the pressure $p$ in his work; both are connected through the equation of state for dilute gases $p=\rho T$.
} 
In this thesis we study two models for the kinetics of the collisions: $(i)$ the simplest model of a constant coefficient of restitution, and $(i i)$ a more realistic model where particles are treated as viscoelastic spheres [27-29] and $\varepsilon$ depends on the impact velocity of the colliding particles. For a realistic granular gas $(\varepsilon=\varepsilon(u))$ the slope of Haff's law changes to $\alpha=5 / 3$ [28]. The hydrodynamic equations, generated through the Chapman-Enskog procedure or Grad's method of moments, for example, are in principle an infinite hierarchy of partial differential equations. In this thesis we study the hydrodynamic equations at the Navier-Stokes (NS) level. The NS equations approximate the transport coefficients within the hydrodynamic equations up to a linear order in the gradients of the variables $(\rho, \boldsymbol{v}, T)$. The corresponding second and third order equations are the Burnett and the super-Burnett equations; both are beyond the scope of this thesis which is due to the fact that the numerical treatment of higher order equations require specialized techniques. DNS of the hydrodynamic equations give access to enlightening results because all hydrodynamic fields are accessible at each point of space and time. Therefore, DNS allows for the possibility of observing fluctuations and structure formation on scales inaccessible to MD simulations. The fundamental theory for the hydrodynamic behavior has been developed in the last three decades for dilute granular systems [30], nevertheless dense granular systems, such as granular assemblies, are far less understood. In MD simulations of granular gases one has to pay attention to statistical averaging because the steadily changing average properties and the strong spatial gradients make any averaging scheme challenging. Appropriate coarse-graining techniques for MD can be found in [31-33]. The big advantage of DNS is that it has direct access to all statistical quantities because of the field theory on which it is based.

\subsection{Content of thesis}

The structure of this thesis is the following. We start with a brief introduction into the kinetic theory of granular gases in Chapter 2 and we introduce the coefficient of restitution $\varepsilon$ as the important parameter to describe the dissipative nature of granular particles. There we show how to derive the NS equations for granular gases which are the fundamental set of equations we study here. We discuss the more realistic problem of viscoelastic particles in Chapter 2 and compare the standard model for viscoelastic spheres with a simple alternative model.

If the system-size $L$ is larger than a critical size

$$
L_{c r i t} \propto\left(1-\varepsilon^{2}\right)^{-1 / 2},
$$

a transition from the homogeneous cooling state (HCS) into an inhomogeneous cooling state (ICS) $[34,35]$ appears, which is characterized by the formation of dense clusters [2125, 36-38]. However, in reality $\varepsilon$ depends on the impact velocity $u$ [7]. The related linear 
stability analysis is given at the end of Chapter 2 .

In Chapter 3 we derive the dimensionless, quasi-conservative form of the granular NS equations. We discuss the pair correlation functions we have implemented and discuss in Section 3.4 the granular dimensionless numbers: Mach, Reynolds and Prandtl numbers.

The necessity of studying large system sizes and of characterizing fluctuations in regions of sharp gradients in temperature and density, developing into supersonic flow, without ambiguities motivates us to tackle the continuous hydrodynamic equations beyond perturbative schemes. In Chapter 4 we describe in detail our solver to perform DNS. We solve the integrated form of the NS equations on a lattice grid with the help of a finite volume method. The integration is performed via Gaussian integration points and the values at such points are reconstructed with a seventh order weighted essentially nonoscillatory (WENO) method. To solve the coupled equations we implemented an operator splitting method between the convective part and the diffusive part of the equations. At the surface of the finite volumes it is important to calculate the fluxes within the finite volume framework. For the convective fluxes, including the dissipative part, we use a MUSCL-Hancock Method (MHM) and the multi-stage (MUSTA) approach to solve the ensuing Riemann problem. The diffusive flux is solved with the diffusive generalized Riemann problem (dGRP) flux.

Our DNS allow us to study fluctuations in a freely cooling granular gas inaccessible to molecular dynamics simulations. In Chapter 5 we show the results of the study of such fluctuations. We show that there is a universal power law relation between the amount of density fluctuations of a granular gas and the Mach number of the system. This implies that there is no typical timescale inside a freely cooling granular gas and that we can describe the age of a granular gas in a universal way just by means of the Mach number.

In Chapter 6 we step away from the global description of the granular gas and follow a local description of a granular gas. We start with a common heuristic argument for the origin of the clustering inside a freely cooling granular gas. We prove that this heuristic does not hold and show a new understanding for the origin of the clustering inside a freely cooling granular gas. Convective subsonic velocities are nearly conserved while the temperature drops, eventually this leads to a transition to shock waves which are the only reason for clustering. Starting from DNS, we compare our results with MD simulations and present analytical arguments that confirm our finding. We want to stress the point that our findings demonstrate that the textbook understanding of the clustering in freely cooling granular gases is erroneous.

In Chapter 7 we study the free cooling in the more realistic model where particles are treated as viscoelastic spheres [27-29] and

$$
\varepsilon(u)=1-\gamma|u|^{1 / 5}+\frac{3}{5} \gamma^{2}|u|^{2 / 5} \mp \ldots,
$$

where $\gamma$ is a constant depending on material properties. The enormous decrease of granular temperature during the cooling process leads to a drastic decrease of the energy dissipation 
$\left(1-\varepsilon^{2}\right)$, which makes the assumption $\varepsilon=$ const unrealistic. For a realistic granular gas $(\varepsilon=\varepsilon(u))$ the slope of Haff's law in Eq. (1.1) is $\alpha=5 / 3$ [28] and it has been shown that the clustering is a transient phenomenon $[30,39]$. Here we show that the disappearance of clusters in granular gases is in principle a finite-size effect. As the granular temperature decreases the critical system size increases until it reaches the size of the system, upon which the density inhomogeneities dissolve and the system returns to a homogeneous state. However, the strong dependence of the time of homogenization on system size implies that for any amount of dissipation a system size can be found where the gas will remain heterogeneous at any realistic time scale. Our findings imply that in an infinite system, a freely cooling granular gas of viscoelastic spheres will not become homogeneous again.

With our DNS we explore the frontiers of the NS approach. In the outlook Chapter 8 we describe a number of studies which are designed to demonstrate the range of applicability of our solver. Here we show results from driven granular systems. We discuss the topic of how to devise a protocol to homogenize a granular gas starting from an inhomogeneous state, Here we also demonstrate that the granular Leidenfrost effect and convective rolls are reproduced by our solver, which, to the best of the authors knowledge, has not been observed in hydrodynamic simulations before. Similar to what has been done before in experiments and in MD simulations we derive a phase diagram which matches rather well the results from MD simulations and experiments. This proves that even in a situation where the assumptions which lead to the NS equations do not hold anymore, we can still find results which are physical. Finally, in the outlook we demonstrate the effect of a central force on a granular gas, thereby we show possible interesting future projects.

With our contribution we hope to advance the research on the hydrodynamics of granular gases and contribute important insights into the fundamental properties of granular systems that have been unknown up to this point. 


\section{Kinetic theory of granular gases}

In this chapter we present the derivation of the NS equations for dilute granular gases. Here we introduce the collision rule for pairwise collisions and derive from this the Boltzmann equation (BE) for granular gases. From the moments of the BE we derive the hydrodynamic equations and the transport coefficients. The Chapman-Enskog approach leads to an infinite hierarchy of equations; we stop this hierarchy at the NS level. We describe and compare two different models for viscoelastic spheres. The first is directly derived from the BE. The second one is obtained from the NS equations for constant coefficient of restitution $\varepsilon$ and uses the dependence of $\varepsilon$ on the local granular temperature as a proxy for the local collisional velocity. We find that both models show no qualitative differences in the dynamics of granular gases. Finally we present Haff's law as the basis solution of a homogeneous freely cooling granular gas and the linear stability analysis of the freely cooling state.

\subsection{Coefficient of restitution}

The macroscopic dynamics of any many-particle-system in nature is determined by microscopic interactions between particles. That means that the evolution of such a system is determined and can be predicted once the full knowledge of the microscopic state and the forces acting are known ${ }^{1}$. The entire dynamics is then governed by Newton's second law

$$
m_{i} \ddot{\boldsymbol{r}}_{i}=\boldsymbol{F}_{i}\left(\boldsymbol{r}_{1}, \ldots, \boldsymbol{r}_{N}, \boldsymbol{u}_{1}, \ldots, \boldsymbol{u}_{N}\right)
$$

\footnotetext{
${ }^{1}$ We only deal with classical problems here without quantum mechanical effects.
} 
which is a system of $N$ coupled ordinary differential equations. In Eq. (2.1) $\boldsymbol{r}_{i}$ represents the position of particle $i, m_{i}$ its mass, $\boldsymbol{F}_{i}$ the total force acting on it, which in general may depend on all particles' positions $\boldsymbol{r}_{i}$ and velocities $\boldsymbol{u}_{i}, i=1, \ldots, N$, and each dot over $\boldsymbol{r}_{i}$ represents a time derivative. Because this set of equations is practically impossible to solve, for any realistic value of $N$, it becomes necessary to focus on the main physical observables which determine the macroscopic behavior.

In a granular systems it is reasonable to assume that the dynamics is well described by short range interactions, which means that particles experience forces only if they come into contact with other material particles. We assume that the interaction between particles is pairwise additive. This assumption is fulfilled if the interaction time $\tau_{I}$ during particle collisions is small compared with the average time $\tau_{L}$ when particles move along straight lines between one collision and the next. That means the assumption $\tau_{I} \ll \tau_{L}$ is valid as long as the system is dilute enough or the particles are hard enough ${ }^{2}$; that is why this assumption is called the hard-sphere limit. Under this assumption equations (2.1) decouple, due to the fact that we have to care only about two particle collision from now on

$$
m_{i j} \ddot{\boldsymbol{r}}_{i j}=\boldsymbol{F}\left(\boldsymbol{r}_{i j}, \boldsymbol{u}_{i j}\right)
$$

where $\boldsymbol{r}_{i j}=\boldsymbol{r}_{i}-\boldsymbol{r}_{j}$ and $\boldsymbol{u}_{i j}=\boldsymbol{u}_{i}-\boldsymbol{u}_{j}$ is the center of mass velocity. We are left with $\frac{N(N-1)}{2}$ linear dependent one-body problems in Eq. (2.2) for particles of reduced mass $m_{i j}=\frac{m_{i} m_{j}}{m_{i}+m_{j}}$. We want to study a system where particles do not change their shape permanently during interactions. The easiest particle interaction we can think of in this context is an inelastic collision. Due to the fact that the particles are composed of an enormous number of atoms or molecules, there exist a multitude of inner degrees of freedom which can absorb a portion of the kinetic energy of the particles during a collision. It is reasonable to assume that the inner energy of such a granular particle is negligible compared to any macroscopic kinetic energy of it. That means that the kinetic energy during a collision is reduced and lost from our observation, or in other words: the system dissipates energy. The amount of energy dissipated during a collision might depend, even under all our assumptions, on many material parameters, like the roughness, the kind of surface or the compressibility. Even if the entire collision process underlies a lot of material parameters, in total there is only one fraction of energy which is dissipated during a collision. That means that we can describe the collision process by only one parameter, the coefficient of restitution, which is the ratio of the normal component of the relative velocity before $\left(g_{i j}\right)$ and after $\left(g_{i j}^{\prime}\right)$ a collision

$$
\varepsilon_{i j} \equiv-g_{i j}^{\prime} / g_{i j}
$$

We assume here that the tangential relative velocity is conserved. The normal force

\footnotetext{
${ }^{2}$ In a dilute system the time between collisions is very large $\tau_{L} \gg 1$; For hard spheres the interaction time is very short $\tau_{I} \ll 1$.
} 
between contacting particles is determined by the compression and the compression rate of particles. The tangential interaction forces instead are especially effected by surface properties like the roughness of particles. Specialized analysis of the role of the tangential part of $\varepsilon$ can be found in [40,41]. The collision rule for a pairwise collision between two identical particles of mass $m_{p}$ reads as

$$
\begin{aligned}
\boldsymbol{u}_{i}^{\prime} & =\boldsymbol{u}_{i}-\frac{1}{2}(1+\varepsilon)\left(\boldsymbol{u}_{i j} \cdot \boldsymbol{e}_{i j}\right) \boldsymbol{e}_{i j} \\
\boldsymbol{u}_{j}^{\prime} & =\boldsymbol{u}_{j}+\frac{1}{2}(1+\varepsilon)\left(\boldsymbol{u}_{i j} \cdot \boldsymbol{e}_{i j}\right) \boldsymbol{e}_{i j},
\end{aligned}
$$

where $\boldsymbol{e}_{i j}=\frac{\boldsymbol{r}_{i j}}{\left|\boldsymbol{r}_{i j}\right|}$ is the unit vector of the separation of the particles at impact. We used in Eq. (2.4) the identity $g_{i j}=\boldsymbol{u}_{i j} \cdot \boldsymbol{e}_{i j}$. The kinetic energy is given by

$$
\begin{aligned}
& \frac{1}{2} m_{p} \boldsymbol{u}_{i}^{\prime 2}=\frac{1}{2} m_{p}\left[\boldsymbol{u}_{i}^{2}-\left(1+\varepsilon_{i j}\right)\left(\boldsymbol{u}_{i j} \cdot \boldsymbol{e}_{i j}\right) \boldsymbol{u}_{i} \cdot \boldsymbol{e}_{i j}+\frac{1}{4}\left(1+\varepsilon_{i j}\right)^{2}\left(\boldsymbol{u}_{i j} \cdot \boldsymbol{e}_{i j}\right)^{2}\right] \\
& \frac{1}{2} m_{p} \boldsymbol{u}_{j}^{\prime 2}=\frac{1}{2} m_{p}\left[\boldsymbol{u}_{j}^{2}-\left(1+\varepsilon_{i j}\right)\left(\boldsymbol{u}_{i j} \cdot \boldsymbol{e}_{i j}\right) \boldsymbol{u}_{j} \cdot \boldsymbol{e}_{i j}+\frac{1}{4}\left(1+\varepsilon_{i j}\right)^{2}\left(\boldsymbol{u}_{i j} \cdot \boldsymbol{e}_{i j}\right)^{2}\right] .
\end{aligned}
$$

The simplest model which describes the dissipating character of a granular gas is the model of constant coefficient of restitution $\varepsilon$. In this model we treat the coefficient of restitution as a material parameter. However it was shown by Tanaka already in $1983[42]^{3}$ that the assumption of a constant $\varepsilon$ during collisions leads to an unphysical viscosity. Kuwabara suggested from heuristic arguments in 1987 [27] a dependency of $\varepsilon$ on the impact velocity

$$
\varepsilon_{i j} \approx 1-A g_{i j}^{1 / 5}
$$

which was formally derived by Schwager and Poeschel ten years later [28] for viscoelastic spheres. More experimental measurement followed in 1988 [44] and proved the dependency of the coefficient of restitution on the impact velocity. We will focus in the entire thesis on these two models.

Strictly speaking the dependency in Eq. (2.6) was derived for viscoelastic spheres, which means that whenever we use the viscoelastic model in two dimensions (2D) we are really considering three dimensions (3D) spheres confined to a plane. The corresponding $\varepsilon$ dependency for viscoelastic discs [45] for small impact velocities $v_{i j}$ and small viscous relaxation time $\alpha \ll 1$ reads to the first order in $\alpha$ as

$$
\varepsilon_{i j} \approx 1-\alpha \sum_{k=0}^{\infty} C_{k}\left[-W_{0}\left(-2 e u_{i j}^{2}\right)\right]^{-k-1 / 2},
$$

where $e$ is the Euler constant and $W_{0}(x)$ is the Lambert $W$ function for values larger than -1 . Tha Lambert $W$ function satisfy the implicit equation

$$
x=W(x) \mathrm{e}^{W(x)}, W \geq-1 .
$$

\footnotetext{
${ }^{3}$ See Taguchi [43] for an English translation.
} 
Because of the complicated form of Eq. $(2.7)^{4}$ and because the results of the experiments are too noisy [44] to prove the $2 \mathrm{D}$ dependency in Eq. (2.7), we keep the easier 3D viscoelastic sphere model to simulate the dissipative behavior of the grains. From Eq. (2.6) it can be seen that the coefficient of restitution does not vary too much if the velocity does not change of orders of magnitude, so that quite often the easier model of treating $\varepsilon$ as a material property is used. Results from MD simulations using the model of constant $\varepsilon$ do in fact reproduce numerous experimental results [2, 46, 47].

Finally, we should keep in mind that we neglect charging effects as well as self-gravity, which might be important in similar systems, especially if one realizes that self-charging of particles is nearly inevitable [48]. Strictly speaking there is a range of phenomena which affect $\varepsilon$; a numerical and experimental study on a resulting distribution of $\varepsilon$ can be found in [49].

\subsection{Coefficient of restitution in the viscoelastic model}

We want to discuss below how it is possible to use a variable coefficient of restitution in a field theory, which will be necessary if we want to build a direct numerical solver for the hydrodynamic equations of viscoelastic spheres. In the viscoelastic model we can express the coefficient of restitutions dependence on the impact velocity [28]

$$
\varepsilon_{i j}=1-C_{1} \tilde{\kappa} g_{i j}^{1 / 5}+C_{2} \tilde{\kappa}^{2} g_{i j}^{2 / 5}+\mathcal{O}\left(\tilde{\kappa}^{3} g_{i j}^{3 / 5}\right)
$$

where $g_{i j}$ is the normal impact velocity between two particles, $\tilde{\kappa}$ is a parameter which contains material properties (e.g. the Young modulus and the Poisson ratio), $C_{1} \approx$ $\frac{\sqrt{\pi}}{2^{1 / 5} 5^{2 / 5}} \frac{\Gamma(3 / 5)}{\Gamma(21 / 10)} \approx 1.15344, C_{2}=\frac{3}{5} C_{1}^{2} \approx 0.79826$, and $\mathcal{O}\left(\tilde{\kappa}^{3} g_{i j}^{3 / 5}\right)$ represents terms of order $\tilde{\kappa}^{3} g_{i j}^{3 / 5}$ or higher. The concrete expression of $\tilde{\kappa}$ is not important for our model; for more details on the derivation of the model and the concrete expression of $\tilde{\kappa}$ see $[28,30]$. We introduce the local velocity of particle $i$

$$
\boldsymbol{\omega}_{i}=\boldsymbol{u}_{i}-\langle\boldsymbol{u}\rangle
$$

where $\langle\boldsymbol{u}\rangle$ is the average velocity within the small volume. We define the local granular temperature and thermal velocity

$$
\begin{array}{r}
\frac{d}{2} T=\frac{1}{2} m_{p}\left\langle\omega^{2}\right\rangle, \\
v_{t h} \equiv \sqrt{\frac{2 T}{m_{p}}}
\end{array}
$$

\footnotetext{
${ }^{4}$ Here complicated means especially that the additional computational cost of calculating such a function does not produce appreciably different results.
} 
and rewrite the impact velocity $g_{i j}$ in terms of the thermal velocity $g_{i j}=v_{t h} c_{i j}$, where $c_{i j}=\left|\boldsymbol{c}_{i j} \cdot \boldsymbol{e}_{i j}\right|=\left|\frac{\boldsymbol{u}_{i j}}{v_{t h}} \cdot \boldsymbol{e}_{i j}\right|$ is the scaled fraction of the normal impact velocity. We rewrite $\varepsilon$ in terms of the scaled variable $c_{i j}$

$$
\begin{aligned}
\varepsilon_{i j} & =1-C_{1} \delta^{\prime} c_{i j}^{1 / 5}+C_{2} \delta^{\prime 2} c_{i j}^{2 / 5}+\mathcal{O}\left(\delta^{\prime 3} c_{i j}^{3 / 5}\right) \\
\delta^{\prime} & =\tilde{\kappa}\left(\frac{2 T}{m_{P}}\right)^{1 / 10} .
\end{aligned}
$$

For a field theory we have to define $\varepsilon$ locally. We use the assumption that only the local thermal velocity defines the local $\varepsilon$, that means $\left|\boldsymbol{u}_{i}\right|=\left|\boldsymbol{u}_{j}\right|=v_{t h}$ and $\langle u\rangle=0$, and we assume that there is no preferred collision direction $\left\langle u_{i j}\right\rangle=0$. In this context the local thermal velocity means that we find a small volume in space, where the granular temperature is homogeneous. We also assume that we have at this local position enough particles to fulfill the central limit theorem. Then we can calculate the expectation values $\mathbb{E}\left(c_{i j}^{1 / 5}\right)$ and $\mathbb{E}\left(c_{i j}^{2 / 5}\right)$ as

$$
\begin{aligned}
\mathbb{E}\left(c_{i j}^{1 / 5}\right) & =\mathbb{E}\left(\left|\boldsymbol{c}_{i j} \cdot \boldsymbol{e}\right|^{1 / 5}\right) \\
& =\mathbb{E}\left(\left|\boldsymbol{c}_{i j}\right|^{1 / 5} \cos ^{1 / 5}(\theta)\right), \quad \theta \in[0, \pi / 2] \\
& =\mathbb{E}\left(\left[(1-\cos (\phi))^{2}+\sin ^{2}(\phi)\right]^{1 / 10} \cos ^{1 / 5}(\theta)\right), \quad \theta \in[0, \pi / 2], \phi \in[0, \pi] \\
& =\frac{1}{\pi} \frac{1}{\pi / 2} \int_{0}^{\pi} \int_{0}^{\pi / 2}\left[(1-\cos (\phi))^{2}+\sin ^{2}(\phi)\right]^{1 / 10} \cos ^{1 / 5}(\theta) \mathrm{d} \phi \mathrm{d} \theta \\
& =\frac{2^{1 / 5}}{\pi} \frac{\Gamma\left(\frac{3}{5}\right)^{2}}{\Gamma\left(\frac{11}{10}\right)^{2}} \approx 0.895935,
\end{aligned}
$$

and

$$
\begin{aligned}
\mathbb{E}\left(c_{i j}^{2 / 5}\right) & =\frac{1}{\pi} \frac{1}{\pi / 2} \int_{0}^{\pi} \int_{0}^{\pi / 2}\left[(1-\cos (\phi))^{2}+\sin ^{2}(\phi)\right]^{2 / 10} \cos ^{2 / 5}(\theta) \mathrm{d} \phi \mathrm{d} \theta \\
& =\frac{2^{2 / 5}}{\pi} \frac{\Gamma\left(\frac{7}{10}\right)^{2}}{\Gamma\left(\frac{6}{5}\right)^{2}} \approx 0.839467,
\end{aligned}
$$

where $\Gamma(x)=\int_{0}^{\infty} t^{x-1} \mathrm{e}^{-t} \mathrm{~d} t$ is Euler's gamma function.

We replace the scaled impact velocity $c_{i j}$ by its mean and find the dependence of $\varepsilon$ on the rescaled temperature $\delta^{\prime}$

$$
\varepsilon=1-\tilde{C}_{1} \delta^{\prime}+\tilde{C}_{2} \delta^{\prime 2}+\mathcal{O}\left(\delta^{\prime 3}\right),
$$

where $\tilde{C}_{1}=\mathbb{E}\left(c_{i j}^{1 / 5}\right) C_{1}, \tilde{C}_{2}=\mathbb{E}\left(c_{i j}^{2 / 5}\right) C_{2}$. We use Eq. (2.16) to define a local $\varepsilon$. If we know $\varepsilon\left(T_{0}\right)=\varepsilon_{0}$ at a reference temperature $T_{0}$ we can rewrite Eq. (2.16); at the linear and quadratic order this reads

$$
\varepsilon^{I}(T)=1-\left(1-\varepsilon_{0}\right)\left(T / T_{0}\right)^{1 / 10}
$$




$$
\varepsilon^{I I}(T)=1+\tilde{A} T^{1 / 10}+\frac{9}{25} C_{1} \tilde{A}^{2} T^{2 / 10}
$$

where $\tilde{A}=\frac{25}{18 C_{1}^{2}}\left(-1-\sqrt{1-\left(\frac{6 C_{1}}{5}\right)^{2}\left(1-\varepsilon_{0}\right)}\right)$. In our simulations we use $\varepsilon^{I}$ because of superior performance.

\subsection{Knudsen number}

When deriving a hydrodynamic theory of granular gases it is important to think about the limitations of such a theory. To derive the NS equations it is necessary that the mean free path $\lambda$ of the particles is sufficiently small compared to any characteristic length scale $L$ inside the system. The ratio between these quantities is known as the Knudsen number $[50]$

$$
\mathcal{K} \equiv \frac{\lambda}{L}
$$

which is the important dimensionless parameter to validate the NS equations. Heuristically, the important role of the Knudsen can be shown by the following example. Let's assume that we have a field theory in which the field $\phi(\hat{r})$ depends on space (we neglect the time dependency here and present the problem in one dimension). We introduce the dimensionless length $r=\frac{\hat{r}}{L}$. The field theory is valid, if the value of $\phi$ does not vary too much in the distance of the mean free path, which means that we can express $\phi(r+\lambda / L)$ in a Taylor series around $r$

$$
\phi(r+\lambda / L)=\phi(r)+\partial_{r} \phi(r) \mathcal{K}+\frac{1}{2} \partial_{r r} \phi(r) \mathcal{K}^{2}+\mathcal{O}\left(\mathcal{K}^{3}\right) .
$$

It is clear that the larger the Knudsen number the more coefficients in Eq. (2.20) have to be taken into account; this strictly means that the hydrodynamic equations are only valid in the limit $\mathcal{K} \ll 1$. The hydrodynamic regime is valid at values $\mathcal{K} \leq 0.01$ [50], which are well described by the NS equations [50].

To obtain $\mathcal{K}$ the mean free path $\lambda$ has to be known. We use below a common, simple heuristic to obtain $\lambda[30,50]$. We consider a reference particle with a diameter $\sigma$ and a velocity $\bar{u}_{p}$ as shown in Fig. 2.1. For reasons of simplicity the other particles are at rest. During the small time $\Delta t$ the reference particle may collide with all the particles which have their centers of mass within the colliding cylinder of volume $\pi \sigma^{2} \bar{u} \Delta t$. The number of collisions follows by multiplying the volume with the number density $\rho$. Now we search for the time $\tau_{m f}$ at which one collision occurs, which is called the mean free time

$$
\tau_{m f}=\frac{1}{\pi \sigma^{2} \bar{u} \rho}
$$




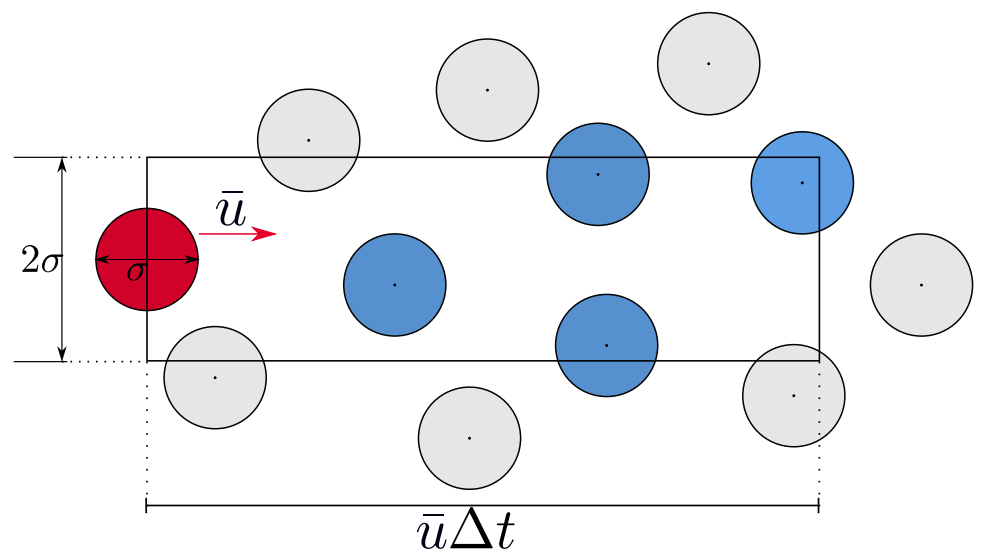

Figure 2.1: Collision cylinder of a particle. We assume that during a short $\Delta t$ the particle will collide with all particles, which have their centers of mass within the colliding cylinder of volume $\pi \sigma^{2} \bar{u} \Delta t$.

the inverse $\frac{1}{\tau_{m} f}$ is called the collision frequency. We find the mean free path from the distance a particle moves during $\tau_{m f}$, which is given by

$$
\lambda=\tau \bar{u}=\frac{1}{\pi \sigma^{2} \rho}
$$

With $\rho=\frac{\phi}{\frac{4}{3} \pi(\sigma / 2)^{3}}$, where $\phi$ is the filling fraction, we get the mean free path

$$
\lambda=\frac{\sigma}{6 \phi}
$$

The generalization of this idea gives the Knudsen number in two and three dimension

$$
\mathcal{K}=\frac{\sigma}{\phi L} D(d)
$$

where $D(3)=\frac{1}{6} ; D(2)=\frac{\pi}{8}$ are constants which depends on the dimensionality $d$.

Now the question should rise if typically granular systems fulfill the hydrodynamic assumption of small $\mathcal{K}$. In typical experiments we find particle diameters from $100 \mu \mathrm{m}$ to 1 cm and average filling fractions $0.01 \leq \bar{\phi} \leq 0.1[15,46,47]$. Most experiments in a lab use system sizes around $10 \mathrm{~cm}$, which leads to a range of Knudsen numbers $0.001 \leq \mathcal{K} \leq 0.1$. Of course, this is a pretty rough estimation but it clarifies that even for granular particles we find setups that realize the hydrodynamic limit of small Knudsen numbers, which gives confidence in the hydrodynamic model of granular gases.

We remark that this simple heuristic does not hold if we assume more problematic systems containing long range electrostatic forces like dipole moments, charging [48] or gravitational forces in astrophysical processes. 


\subsection{Velocity distribution function}

To derive a field theory for a granular system we have to start with the particles in the phase space, which is described by the locations $\boldsymbol{r}_{i}$ and the velocities $\boldsymbol{u}_{i}$ of the particles. Consider an infinitesimal volume of phase space of size $\mathrm{d} \boldsymbol{r} \mathrm{d} \boldsymbol{u}$ and ask how many particles have positions and velocities. We thus need to consider the probability density function $f(\boldsymbol{r}, \boldsymbol{u}, t)$ in phase space. The phase space density $f(\boldsymbol{r}, \boldsymbol{u}, t)$ is called the velocity distribution function [30] and its integral over a fixed phase-space volume $\Omega$

$$
N_{\Omega}=\int_{\Omega} f(\boldsymbol{r}, \boldsymbol{u}, t) \mathrm{d} \boldsymbol{r} \mathrm{d} \boldsymbol{u}
$$

is the number of particles within $\Omega$. If we integrate over the entire phase space we obtain the number of particles $N$ inside our system. Because of the interactions between particles, $f(\boldsymbol{r}, \boldsymbol{u}, t)$ will dynamically change over time. We point out that, physically, the volume $\mathrm{d} \boldsymbol{r} \mathrm{d} \boldsymbol{u}$ has to be a small phase-space volume from a macroscopic point of view, but must be so large that it contains enough particles to define macroscopic quantities. Apart from $f$ itself, also the moments of $f$ are very helpful because they are directly related to macroscopic properties of the gas; the first three moments in $d$-dimensions read as

$$
\begin{aligned}
\rho(\boldsymbol{r}, t) & =\int f(\boldsymbol{r}, \boldsymbol{u}, t) \mathrm{d} \boldsymbol{u} \\
\rho \boldsymbol{v}(\boldsymbol{r}, t) & =\int \boldsymbol{u} f(\boldsymbol{r}, \boldsymbol{u}, t) \mathrm{d} \boldsymbol{u}, \\
\frac{d}{2} \rho T(\boldsymbol{r}, t) & =\int \frac{1}{2} m_{p} \boldsymbol{w}^{2} f(\boldsymbol{r}, \boldsymbol{u}, t) \mathrm{d} \boldsymbol{u},
\end{aligned}
$$

where $\rho$ is the number density, $\rho \boldsymbol{v}=\rho\langle\boldsymbol{u}\rangle$ is the momentum density,

$$
\boldsymbol{w}=\boldsymbol{u}-\boldsymbol{v}
$$

is the local or thermal velocity and $T$ is the granular temperature of the gas. The time evolution of $f$ is thereby of main interest. The equation which describes the time evolution of $f$ is called the BE and was first introduced by Ludwig Boltzmann in 1896. The BE is the fundamental equation within the kinetic gas theory.

\subsection{Boltzmann equation}

We start by observing a systems which contains $N$ particles

$$
N=\int_{\mathbb{R}^{6}} f(\boldsymbol{r}, \boldsymbol{u}, t) \mathrm{d} \boldsymbol{r} \mathrm{d} \boldsymbol{u}
$$


We assume that the number of particles is conserved, even if density sinks/sources can be easily included in the theory. Due to the collision of particles the system dissipates energy. But because the number of particles does not change, $f$ has to fulfill the continuity equation

$$
\partial_{t} f=-\partial_{\boldsymbol{r}, \boldsymbol{u}} \cdot(\dot{\xi} f)
$$

where $\partial_{\boldsymbol{r}, \boldsymbol{u}}=\nabla_{\boldsymbol{r}, \boldsymbol{u}}$ is the nabla operator with respect to $\boldsymbol{r}$ and $\boldsymbol{u}$ and with the phase-space velocity $\dot{\xi}=\{\dot{\boldsymbol{r}}, \dot{\boldsymbol{u}}\}=\{\boldsymbol{u}, \dot{\boldsymbol{u}}\}$. The dissipation of energy leads to a compression of $f$, which means that the phase-space area of high velocities $\boldsymbol{u}$ becomes more and more dilute over time, while the phase-space around zero velocity becomes more dense over time. If we expand the components in the gradient in Eq. (2.29) we find

$$
\partial_{t} f+\boldsymbol{u} \cdot \partial_{r} f+\partial_{\boldsymbol{u}}(\dot{\boldsymbol{u}} f)=0
$$

since $\boldsymbol{r}$ and $\boldsymbol{u}$ are independent variables in phase-space the gradient $\partial_{\boldsymbol{r}} \boldsymbol{u}$ in Eq.(2.30) vanishes. The acceleration $\dot{\boldsymbol{u}}$ in Eq. (2.30) can be split in a term which results out of external forces $\boldsymbol{G}$, e.g. gravity or vibrating, solid walls, and a term $\boldsymbol{W}$ due to the interaction forces between the particles, $\dot{\boldsymbol{u}}=\boldsymbol{G}+\boldsymbol{W}$. We finally obtain the Boltzmann equation

$$
\partial_{t} f+\boldsymbol{u} \cdot \partial_{r} f+\boldsymbol{G} \cdot \partial_{\boldsymbol{u}} f=I
$$

where we call $I=-\partial_{\boldsymbol{u}} \cdot(\boldsymbol{W} f)$ the collision integral. We assume that the external forces $\boldsymbol{G}$ in Eq. (2.31) are independent of the velocity, the generalization to velocity-dependent external forces is doable but not necessary within the scope of this thesis.

The details of the particle-particle interactions define the collision integral. In the following argumentation we use assumptions which are known as "Stosszahlansatz" and are valid for dilute gases [50]: $(i)$ We assume only binary collisions, which corresponds to the so called hard-sphere limit. The assumption of binary collision is fulfilled to a good approximation if the gas is dilute or if the collision time $\alpha$ is small enough; the limit $\alpha \rightarrow 0$ is fulfilled for ideal hard spheres. (ii) The velocity distribution function does not change significantly after one collision. ( $i i i) f$ varies in space but is constant over the range of interparticle interactions. ( $i v$ ) The correlation between different velocities $u_{i}$ before a collision are neglected (hypothesis of molecular chaos).

To calculate the collision integral $I=I^{+}-I^{-}$we have to find the number of collisions per unit time that produce a phase point $\left(I^{+}\right)$and that destroy a phase point $\left(I^{-}\right)$because of internal collisions [30]. We assume for now a constant coefficient of restitution and observe the number of particles $f\left(\boldsymbol{r}_{i}, \boldsymbol{u}_{i}, t\right) \mathrm{d} \boldsymbol{r}_{i} \mathrm{~d} \boldsymbol{u}_{i}$ in the volume $\mathrm{d} \boldsymbol{r}_{i} \mathrm{~d} \boldsymbol{u}_{i}$ located at $\left(\boldsymbol{r}_{i}, \boldsymbol{u}_{i}\right)$. Typically a collision involving a particle out of this phase-space volume will change the velocity and thereby the particle will leave the volume element $\mathrm{d} \boldsymbol{r}_{i} \mathrm{~d} \boldsymbol{u}_{i}$. Such collisions are called direct collision and will reduce the phase-space density $f$ at $\left(\boldsymbol{r}_{i}, \boldsymbol{u}_{i}\right)$. The collision 
law for a direct collision is given by Eq. (2.4)

$$
\begin{aligned}
& \boldsymbol{u}_{i}^{\prime}=\boldsymbol{u}_{i}-\frac{1+\varepsilon}{2}\left(\boldsymbol{u}_{i j} \cdot \boldsymbol{e}_{i j}\right) \boldsymbol{e}_{i j} \\
& \boldsymbol{u}_{j}^{\prime}=\boldsymbol{u}_{j}+\frac{1+\varepsilon}{2}\left(\boldsymbol{u}_{i j} \cdot \boldsymbol{e}_{i j}\right) \boldsymbol{e}_{i j} .
\end{aligned}
$$

To obtain the number of direct collisions we have to count all collisions occurring in a small time $\Delta t$ at $d \boldsymbol{r}_{i}$ [30]. We assume that all collisions occur independently, so that we find a small cylinder in phase space in which a particle will collide during $\Delta t$ with a neighbor within $\mathrm{d} \boldsymbol{r}_{i} \mathrm{~d} \boldsymbol{u}_{i}$. The cylinder has height $\Delta t\left|\boldsymbol{u}_{i j} \cdot \boldsymbol{e}_{i j}\right|$ and a base of $\sigma^{2} \mathrm{~d} \Omega$ where $r_{i j}=\sigma$ during a collision and $\mathrm{d} \Omega$ is the solid angle around $\boldsymbol{e}_{i j}$. If we multiply the volume of the collision cylinder by the density $f\left(\boldsymbol{r}_{i}, \boldsymbol{u}_{i}\right) \mathrm{d} \boldsymbol{r}_{i} \mathrm{~d} \boldsymbol{u}_{i}$ of the phase space

$$
\mathrm{d} N_{C C}=f\left(\boldsymbol{r}_{i}, \boldsymbol{u}_{i}, t\right) \mathrm{d} \boldsymbol{r}_{i} \mathrm{~d} \boldsymbol{u}_{i} \Delta t\left|\boldsymbol{u}_{i j} \cdot \boldsymbol{e}_{i j}\right| \sigma^{2} \mathrm{~d} \Omega \mathrm{d} \boldsymbol{u}_{i}
$$

we obtain the number of particles with velocity $\boldsymbol{u}_{i}$ within this cylinder. Finally, we obtain the number of direct collisions $\nu^{-}$at $\boldsymbol{r}_{i}$ with velocities $\boldsymbol{u}_{i}$ and $\boldsymbol{u}_{j}$ that represent a loss of phase-space points from the volume $\left(\boldsymbol{r}_{i}, \boldsymbol{u}_{i}\right)$ if we multiply the number of particles $\mathrm{d} N_{C C}$ by the phase-space density $f\left(\boldsymbol{r}_{i}, \boldsymbol{u}_{j}\right)$

$$
\nu^{-}=f\left(\boldsymbol{r}_{i}, \boldsymbol{u}_{i}, t\right) f\left(\boldsymbol{r}_{i}, \boldsymbol{u}_{j}, t\right) \mathrm{d} \boldsymbol{r}_{i} \mathrm{~d} \boldsymbol{u}_{i} \mathrm{~d} \boldsymbol{u}_{j} \Delta t\left|\boldsymbol{u}_{i j} \cdot \boldsymbol{e}_{i j}\right| \sigma^{2} \mathrm{~d} \Omega .
$$

Due to collisions $f\left(\boldsymbol{r}_{i}, \boldsymbol{u}_{i}\right)$ increases in the case that particles with initial velocities $\boldsymbol{u}_{i}^{\prime \prime}, \boldsymbol{u}_{j}^{\prime \prime}$ acquire velocities $\boldsymbol{u}_{i}, \boldsymbol{u}_{j}$ after the collision; we call them inverse collision. The collision rule reads as

$$
\begin{aligned}
\boldsymbol{u}_{i} & =\boldsymbol{u}_{i}^{\prime \prime}-\frac{1+\varepsilon}{2}\left(\boldsymbol{u}_{i j}^{\prime \prime} \cdot \boldsymbol{e}_{i j}\right) \boldsymbol{e}_{i j} \\
\boldsymbol{u}_{j} & =\boldsymbol{u}_{j}^{\prime \prime}+\frac{1+\varepsilon}{2}\left(\boldsymbol{u}_{i j}^{\prime \prime} \cdot \boldsymbol{e}_{i j}\right) \boldsymbol{e}_{i j} .
\end{aligned}
$$

With $\boldsymbol{u}_{i j} \cdot \boldsymbol{e}_{i j}=-\varepsilon \boldsymbol{u}_{i j}^{\prime \prime} \cdot \boldsymbol{e}_{i j}$ and a bit of algebra we find $\mathrm{d} \boldsymbol{u}_{i}^{\prime \prime} \mathrm{d} \boldsymbol{u}_{j}^{\prime \prime}=\frac{1}{\varepsilon} \mathrm{d} \boldsymbol{u}_{i} \mathrm{~d} \boldsymbol{u}_{j}[30]$. The number of inverse collision $\nu^{+}$follows from the same arguments as the number of direct collisions $\nu^{-}$and can be written as [30]

$$
\nu^{+}=\frac{1}{\varepsilon^{2}} \nu^{-}
$$

We can finally write the collision integral $I$ in Eq. (2.31)

$$
I(f, f)=\sigma^{2} \int \mathrm{d} \boldsymbol{u}_{j} \int \mathrm{d} \boldsymbol{e}_{i j} \Theta\left(-\boldsymbol{u}_{i j} \cdot \boldsymbol{e}_{i j}\right)\left|\boldsymbol{u}_{i j} \cdot \boldsymbol{e}_{i j}\right|\left[\frac{1}{\varepsilon^{2}} f\left(\boldsymbol{u}_{i}^{\prime \prime}\right) f\left(\boldsymbol{u}_{j}^{\prime \prime}\right)-f\left(\boldsymbol{u}_{i}\right) f\left(\boldsymbol{u}_{j}\right)\right],
$$

where $\Theta(x)$ is the Heaviside function. Typically the collision integral $I$ is multiplied by the pair correlation function at contact $g_{2}(\sigma)$ to exclude volume effects, where $g_{2}(\sigma)$ is 
also known as the Enskog factor. The modified BE

$$
\partial_{t} f+\boldsymbol{u} \cdot \nabla_{\boldsymbol{r}} f+\boldsymbol{G} \cdot \nabla_{\boldsymbol{u}} f=g_{2}(\sigma) I
$$

is called the Boltzmann-Enskog equation. The collision integral for viscoelastic particles has a more complicated shape, the derivation and the formula can be found in $[30,51]$.

\subsection{Hydrodynamic equations}

We follow the textbook method in $[30,50]$ to derive the hydrodynamic equations. That means we have to calculate the moments of the velocity distribution function in Eq. (2.26). The evolution of the moments can be derived from the BE, which leads us to the hydrodynamic equations. Given a function $\psi(\boldsymbol{r}, \boldsymbol{u}, t) \equiv \psi(\boldsymbol{u})$, the average value is given by $\langle\psi(t)\rangle \equiv$ $\int \psi(\boldsymbol{u}) f(\boldsymbol{u}, t) \mathrm{d} \boldsymbol{u}$ and the change of the variable is given by $\Delta \psi\left(\boldsymbol{u}_{i}\right) \equiv\left[\psi\left(\boldsymbol{u}_{i}^{\prime}\right)-\psi\left(\boldsymbol{u}_{i}\right)\right]$. The collision integral in Eq. (2.37) has now the important property [30]

$$
\begin{aligned}
\frac{\mathrm{d}}{\mathrm{d} t}\langle\psi(t)\rangle & =\int \mathrm{d} \boldsymbol{u} \psi(\boldsymbol{u}) \partial_{t} f(\boldsymbol{u})=g_{2} \int \mathrm{d} \boldsymbol{u} \psi(\boldsymbol{u}) I(f, f) \\
& =\frac{1}{2} \sigma^{2} g_{2} \int \mathrm{d} \boldsymbol{u}_{i} \mathrm{~d} \boldsymbol{u}_{j} \int \mathrm{d} \boldsymbol{e} \Theta\left(-\boldsymbol{u}_{i j} \cdot \boldsymbol{e}_{i j}\right)\left|\boldsymbol{u}_{i j} \cdot \boldsymbol{e}_{i j}\right| f\left(\boldsymbol{u}_{i}\right) f\left(\boldsymbol{u}_{j}\right) \Delta\left[\psi\left(\boldsymbol{u}_{i}\right)+\psi\left(\boldsymbol{u}_{j}\right)\right]
\end{aligned}
$$

We multiply the BE in Eq. (2.38) by $\psi(\boldsymbol{u})$ and integrate over $\boldsymbol{u}_{i}$

$$
\int \psi\left(\boldsymbol{u}_{i}\right) \partial_{t} f \mathrm{~d} \boldsymbol{u}_{i}+\int \psi\left(\boldsymbol{u}_{i}\right) \boldsymbol{u}_{i} \cdot \nabla_{\boldsymbol{r}} f \mathrm{~d} \boldsymbol{u}_{i}+\int \psi\left(\boldsymbol{u}_{i}\right)\left(\boldsymbol{G} \cdot \nabla_{\boldsymbol{u}_{i}} f\right) \mathrm{d} \boldsymbol{u}_{i}=\int \psi\left(\boldsymbol{u}_{i}\right) g_{2}(\sigma) I \mathrm{~d} \boldsymbol{u}_{i} .
$$

The right hand side (RHS) of Eq.(2.40) is given by Eq. 2.39. Now we consider the three cases where $\psi(\boldsymbol{u})$ is equal to $1, \boldsymbol{u}$ or $\frac{1}{2} m_{p} \boldsymbol{u}^{2}$ to obtain the moments. By replacing $\psi\left(\boldsymbol{u}_{i}\right)$ with each element of the set $\left\{1, \boldsymbol{u}, \frac{1}{2} m_{p} u^{2}\right\}$, because of the following results

$$
\begin{aligned}
\Delta[1+1] & =1+1-1-1=0 \\
\Delta\left[m_{p} \boldsymbol{u}_{i}+m_{p} \boldsymbol{u}_{j}\right] & =m_{p} \boldsymbol{u}_{i}^{\prime}-m_{p} \boldsymbol{u}_{i}+m_{p} \boldsymbol{u}_{j}^{\prime}-m_{p} \boldsymbol{u}_{j}=0 \\
\Delta\left[m_{p} \boldsymbol{u}_{i}^{2}+m_{p} \boldsymbol{u}_{j}^{2}\right] & =m_{p} \boldsymbol{u}_{i}^{\prime 2}-m_{p} \boldsymbol{u}_{i}^{2}+m_{p} \boldsymbol{u}_{j}^{\prime 2}-m_{p} \boldsymbol{u}_{j}^{2}=-m_{p}\left(1-\varepsilon^{2}\right)\left(\boldsymbol{u}_{i j} \cdot \boldsymbol{e}_{i j}\right)^{2}
\end{aligned}
$$

the integral over the collision integral has only a non-vanishing term for $\psi=\frac{1}{2} m_{p} \boldsymbol{u}^{2}$. The second equation in Eq. (2.41) follows directly from the collision rule in Eq. (2.4), the third equation follows from the energy change during a collision in Eq. (2.5). We calculate now 
the left hand side (LHS) of Eq. (2.40). For $\psi(\boldsymbol{u})=1$, it follows

$$
\begin{aligned}
& \int 1 \cdot \partial_{t} f \mathrm{~d} \boldsymbol{u}=\partial_{t} \int f \mathrm{~d} \boldsymbol{u}=\partial_{t} \rho, \\
& \int 1 \cdot \boldsymbol{u} \cdot \nabla_{\boldsymbol{r}} f \mathrm{~d} \boldsymbol{u}=\nabla_{\boldsymbol{r}} \cdot \int \boldsymbol{u} f \mathrm{~d} \boldsymbol{u}=\nabla_{\boldsymbol{r}} \cdot(\rho \boldsymbol{v}), \\
& \int 1 \cdot\left(\boldsymbol{G} \cdot \nabla_{\boldsymbol{u}} f\right) \mathrm{d} \boldsymbol{u}=\boldsymbol{G} \cdot \int \nabla_{\boldsymbol{u}} f \mathrm{~d} \boldsymbol{u}=0,
\end{aligned}
$$

where we used the moments in Eq. (2.26) and the assumption that $\boldsymbol{G}$ is independent of the velocities. We introduce the notation $\boldsymbol{a} \boldsymbol{b}=\boldsymbol{a} \cdot \boldsymbol{b}^{\top}$, which creates the tensor $D_{i j}=a_{i} b_{j}$. With the thermal velocity $\boldsymbol{w}$ in Eq. (2.27), we find for $\psi(\boldsymbol{u})=\boldsymbol{u}$

$$
\begin{aligned}
\int \boldsymbol{u} \cdot \partial_{t} f \mathrm{~d} \boldsymbol{u} & =\partial_{t} \rho \boldsymbol{v}=\rho \partial_{t} \boldsymbol{v}-\boldsymbol{v} \nabla_{\boldsymbol{r}} \cdot(\rho \boldsymbol{v}) \\
\int \boldsymbol{u} \cdot \boldsymbol{u} \nabla_{\boldsymbol{r}} f \mathrm{~d} \boldsymbol{u} & =\nabla_{\boldsymbol{r}} \cdot\left(\int[\boldsymbol{w}+\boldsymbol{v}][\boldsymbol{w}+\boldsymbol{v}] f(\boldsymbol{u}) \mathrm{d} \boldsymbol{u}\right) \\
& =\nabla_{\boldsymbol{r}} \cdot\left(\int \boldsymbol{w} \boldsymbol{w} f(\boldsymbol{u}) \mathrm{d} \boldsymbol{u}+\boldsymbol{v} \boldsymbol{v} \int f(\boldsymbol{u}) \mathrm{d} \boldsymbol{u}+2 \boldsymbol{v} \int \boldsymbol{w} f(\boldsymbol{u}) \mathrm{d} \boldsymbol{u}\right) \\
& =\frac{1}{m_{p}} \nabla_{\boldsymbol{r}} \cdot \underline{\boldsymbol{\Pi}}(\boldsymbol{r}, t)+\nabla_{\boldsymbol{r}} \cdot(\boldsymbol{v} \boldsymbol{v} \rho) \\
\int \boldsymbol{u}\left(\boldsymbol{G} \nabla_{\boldsymbol{u}} f\right) \mathrm{d} \boldsymbol{u} & =-\boldsymbol{G} \int f(\boldsymbol{u}) \mathrm{d} \boldsymbol{u}=-\rho \boldsymbol{G}
\end{aligned}
$$

where we used in the second equation $\int \boldsymbol{w} f(\boldsymbol{u}) \mathrm{d} \boldsymbol{u}=\int \boldsymbol{u} f(\boldsymbol{u}) \mathrm{d} \boldsymbol{u}-\int \boldsymbol{v} f(\boldsymbol{u}) \mathrm{d} \boldsymbol{u}=\mathbf{0}$ and

$$
\begin{aligned}
\underline{\boldsymbol{\Pi}}(\boldsymbol{r}, t) & \equiv \int m_{p} \boldsymbol{w} \boldsymbol{w} f(\boldsymbol{u}) \mathrm{d} \boldsymbol{u} \\
& =\int m_{p}\left(\boldsymbol{w} \boldsymbol{w}-\frac{1}{d} \boldsymbol{w} \cdot \boldsymbol{w} \underline{\mathbf{1}}\right) f(\boldsymbol{u}) \mathrm{d} \boldsymbol{u}+\frac{1}{d} \underline{\mathbf{1}} \int m_{p} \boldsymbol{w}^{2} f(\boldsymbol{u}) \mathrm{d} \boldsymbol{u} \\
& =\int m_{p}\left(\boldsymbol{w} \boldsymbol{w}-\frac{1}{d} \boldsymbol{w} \cdot \boldsymbol{w} \underline{\mathbf{1}}\right) f(\boldsymbol{u}) \mathrm{d} \boldsymbol{u}+\rho T \underline{\mathbf{1}},
\end{aligned}
$$

is the pressure tensor. The diagonal part of $\underline{\boldsymbol{\Pi}}$ is called the hydrostatic pressure $p$, where $p=\rho T$ in the dilute case, the non-diagonal elements are called the deviatoric stress tensor $\Sigma$. For higher filling fraction the correct hydrostatic pressure is given by

$$
p=\rho T\left(1+2^{d-2}(1+\varepsilon) \phi g_{2}\right) .
$$

The derivation of Eq. (2.45) is skipped here and can be found in [52]. We set $\psi(\boldsymbol{u})=$ $\frac{1}{2} m_{p} \boldsymbol{w}^{2}$ and obtain the last set of equation, where the terms on the LHS of Eq. (2.40) read as

$$
\begin{aligned}
\frac{1}{2} m_{p} \int \boldsymbol{w}^{2} \cdot \partial_{t} f \mathrm{~d} \boldsymbol{u} & =\frac{d}{2}\left(\rho \partial_{t} T+\rho \boldsymbol{v} \nabla_{\boldsymbol{r}} T-\nabla_{\boldsymbol{r}} \cdot(\rho \boldsymbol{v} T)\right), \\
\frac{1}{2} m_{p} \int \boldsymbol{w}^{2} \cdot \boldsymbol{u} \nabla_{\boldsymbol{r}} f \mathrm{~d} \boldsymbol{u} & =\frac{d}{2} \nabla_{\boldsymbol{r}} \cdot(\rho \boldsymbol{v} T)+\underline{\boldsymbol{\Pi}}: \nabla_{\boldsymbol{r}} \boldsymbol{v}+\nabla_{\boldsymbol{r}} \cdot \boldsymbol{q}, \\
\frac{1}{2} m_{p} \int \boldsymbol{w}^{2} \cdot\left(\boldsymbol{G} \cdot \nabla_{\boldsymbol{u}} f\right) \mathrm{d} \boldsymbol{u} & =0
\end{aligned}
$$


where the heat flux $\boldsymbol{q}$ is given by

$$
\boldsymbol{q} \equiv \int \frac{1}{2} m_{p} \boldsymbol{w}^{2} \boldsymbol{w} f(\boldsymbol{u}, r, t) \mathrm{d} \boldsymbol{u},
$$

and the tensor product $\underline{\boldsymbol{\Pi}}: \nabla_{\boldsymbol{r}} \boldsymbol{v}=\operatorname{Tr}\left(\underline{\boldsymbol{\Pi}} \cdot \nabla_{\boldsymbol{r}} \boldsymbol{v}\right)$.

Finally if we collect the results in Eqs. (2.41), (2.42), (2.43), (2.46) we obtain the hydrodynamic equations for the so called primitive variables number density $\rho$, convective velocity $\boldsymbol{v}$ and granular temperature $T$ in $d$-dimension

$$
\begin{aligned}
& \partial_{t} \rho+\nabla \cdot(\rho \boldsymbol{v})=0 \\
& \frac{\mathrm{D}}{\mathrm{D} t} \boldsymbol{v}+\frac{1}{\rho m_{p}} \nabla \cdot \underline{\boldsymbol{\Pi}}=\boldsymbol{a} \\
& \frac{\mathrm{D}}{\mathrm{D} t} T+\frac{2}{d \rho} \underline{\boldsymbol{\Pi}}: \nabla \boldsymbol{v}+\frac{2}{d \rho} \nabla \cdot \boldsymbol{q}=-\xi T,
\end{aligned}
$$

where $\underline{\boldsymbol{\Pi}}$ and $\boldsymbol{q}$ are defined in Eq. (2.44), (2.47) and the cooling rate

$$
\xi \equiv-\frac{1}{d} m_{p} g_{2} \frac{1}{\rho T} \int \boldsymbol{u}_{i}^{2} I\left(f\left(\boldsymbol{u}_{i}\right), f\left(\boldsymbol{u}_{j}\right)\right) \mathrm{d} \boldsymbol{u}_{i},
$$

and where we have used the convective or material derivative $\frac{\mathrm{D}}{\mathrm{D} t} \equiv \frac{\partial}{\partial t}+\boldsymbol{v} \cdot \nabla$ and the collision integral $I$ is defined in Eq. (2.37). From now on we will hide the index in the nabla operator if we mean the spacial derivative $\nabla \equiv \nabla_{\boldsymbol{r}}$.

\subsection{Transport coefficients}

The hydrodynamic equations have been derived in general in the last section. The transport coefficients for the hydrodynamic equations are obtained from the stress tensor $\underline{\boldsymbol{\Pi}}$, the heat flux $\boldsymbol{q}$ and the cooling rate $\xi$ which were derived in section 2.6

$$
\begin{aligned}
\underline{\boldsymbol{\Pi}} & \equiv \int m_{p}\left(\boldsymbol{w} \boldsymbol{w}-\frac{1}{d} \boldsymbol{w} \cdot \boldsymbol{w} \underline{\mathbf{1}}\right) f(\boldsymbol{u}) \mathrm{d} \boldsymbol{u}, \\
\boldsymbol{q} & \equiv \int \frac{1}{2} m_{p} \boldsymbol{w}^{2} \boldsymbol{w} f(\boldsymbol{u}, r, t) \mathrm{d} \boldsymbol{u}, \\
\xi & \equiv-\frac{1}{d} m_{p} g_{2} \frac{1}{\rho T} \int \boldsymbol{u}_{1}^{2} I\left(f\left(\boldsymbol{u}_{1}\right), f\left(\boldsymbol{u}_{2}\right)\right) \mathrm{d} \boldsymbol{u}_{1} .
\end{aligned}
$$

To compute the integrals in Eq. (2.50) Chapman and Enskog independently of each other developed a method [53] to expand the phase-space distribution function around an equilibrium state. The local Maxwellian for the thermal or local velocity $\boldsymbol{w}$ [50] is

$$
f_{M}=\rho \frac{1}{(\sqrt{2 \pi T})^{3}} \exp \left(-\frac{w^{2}}{2 T}\right)
$$


Indeed the correct distribution is slightly different, the corrections are expressed in the so called Sonine expansion

$$
f(\boldsymbol{u}, t)=f_{M}(\boldsymbol{w})\left(1+\sum_{p=1}^{\infty} a_{p}(t) S_{p}(\boldsymbol{w})\right)
$$

The second Sonine coefficient

$$
a_{2}=\frac{16(1-\varepsilon)\left(1-2 \varepsilon^{2}\right)}{81-17 \varepsilon+30 \varepsilon^{2}(1-\varepsilon)}
$$

characterizes the first non-trivial correction to the distribution function $f$ ( $a_{1}$ is identically zero as can be easily derived [30]) from its Gaussian form $f_{M}$ in the homogeneous cooling state and was first derived by van Noije and Ernst[54]. We consider the distribution function to first correction throughout this thesis. For more information on the Sonine polynomial expansion to the distribution function in granular gases see $[30,54,55]$.

The expansion of the phase space density $f$ around $f^{(0)}$ is given in terms of a small parameter $\epsilon$

$$
f=f^{(0)}+\epsilon f^{(1)}+\epsilon^{2} f^{(2)}+\ldots
$$

We assume the compatibility condition [50], which contains the assumption that the main variables $(\rho, \boldsymbol{v}, T)$ are the same at any level of expansion in Eq. (2.54). With this condition, it follows

$$
\begin{aligned}
& \rho(\boldsymbol{r}, t)=\int f^{(0)} \mathrm{d} \boldsymbol{v}, \quad \rho v(\boldsymbol{r}, t)=\int \boldsymbol{u} f^{(0)} \mathrm{d} \boldsymbol{u}, \quad \frac{d}{2} \rho T(\boldsymbol{r}, t)=\int \frac{1}{2} m_{p} w^{2} f^{(0)} \mathrm{d} \boldsymbol{u} \\
& 0=\int f^{(\alpha)} \mathrm{d} \boldsymbol{v}, \quad 0=\int \boldsymbol{u} f^{(\alpha)} \mathrm{d} \boldsymbol{u}, \quad 0=\int \frac{1}{2} m_{p} w^{2} f^{(\alpha)} \mathrm{d} \boldsymbol{u}, \quad \alpha \geq 1,
\end{aligned}
$$

however the stress, heat flux and cooling rate depend on the order of the expansion

$$
\begin{aligned}
\Pi_{i j}^{\alpha} & =m_{p} \int \boldsymbol{w}_{i} \boldsymbol{w}_{j} f^{(\alpha)} \mathrm{d} \boldsymbol{u}, \\
\boldsymbol{q}^{\alpha} & =\frac{1}{2} m_{p} \int \boldsymbol{w}^{2} \boldsymbol{w} f^{(\alpha)} \mathrm{d} \boldsymbol{u}, \\
\xi & =-\frac{1}{d} m_{p} g_{2} \frac{1}{\rho T} \int \boldsymbol{u}_{1}^{2} I\left(f^{(\alpha)}\left(\boldsymbol{u}_{1}\right), f^{(\alpha)}\right)\left(\boldsymbol{u}_{2}\right) \mathrm{d} \boldsymbol{u}_{1} .
\end{aligned}
$$

If we use the Chapman-Enskog expansion (2.54) in the conservation laws Eqs. (2.48) we obtain a hierarchy of equations. At zeroth order $\underline{\boldsymbol{\Pi}}$ and $\boldsymbol{q}$ vanish, which leads to the Euler equations for granular gas. At first order we obtain the transport coefficients in Eq. (2.56) till linear order in the derivatives of the hydrodynamic quantities, which is known as the NS level of the equation. The NS equation is the set of equations which we solve in this thesis. The lengthy calculations are found in [18, 21, 56-58]. For the first non-vanishing 
terms, the transport coefficients read as

$$
\begin{aligned}
\Pi_{i j}^{1} & =p \delta_{i j}-\eta\left(\partial_{i} u_{j}+\partial_{j} u_{i}-\frac{2}{d} \delta_{i j} \nabla \cdot \boldsymbol{u}\right)-\eta_{B} \delta_{i j} \nabla \cdot \boldsymbol{u}, \\
\boldsymbol{q}^{1} & =-\kappa \nabla T-\mu \nabla \rho, \\
\xi & =\xi_{0} \xi^{*}
\end{aligned}
$$

where $\partial_{i}=\frac{\partial}{\partial x_{i}}$ and we name $\eta$ the viscosity, $\eta_{B}$ the bulk viscosity, $\kappa$ the thermal conductivity; $\mu$ has no name in the literature and does not appear in ordinary gases. The transport coefficients read as

$$
\begin{aligned}
\eta & =\eta_{0} \eta^{*}, \\
\eta_{B} & =\eta_{0} \eta_{B}^{*}, \\
\kappa & =\kappa_{0} \kappa^{*}, \\
\mu & =\mu_{0} \mu^{*},
\end{aligned}
$$

with

$$
\begin{aligned}
\eta_{0} & =\frac{2+d}{2^{d+1}} \sqrt{\pi m_{p} T}\left(A_{p} g_{2}\right)^{-1} \\
\eta_{B} & =\frac{2^{2 d+1}}{\pi(d+2)} \phi^{2}(1+\varepsilon)\left(1-\frac{1}{16} a_{2}\right), \\
\kappa_{0} & =\frac{d(d+2)^{2}}{2^{2+d}(d-1)} \sqrt{\frac{\pi T}{m_{p}}}\left(A_{p} g_{2}\right)^{-1} \\
\mu_{0} & =\frac{\kappa_{0} T}{\rho} \\
\xi_{0} & =\frac{\rho T}{\eta_{0}}
\end{aligned}
$$

where $A_{p}=2(\sigma / 2)^{d-1} \frac{\pi^{d / 2}}{\Gamma(d / 2)}$ is the surface of a sphere in $d$ dimension and $\Gamma(x)$ is the gamma function. The dimensionless part of the transport coefficients for the model of constant $\varepsilon$ read [30]

$$
\begin{aligned}
\xi^{*} & =\frac{2+d}{4 d}\left(1-\varepsilon^{2}\right)\left(1+\frac{3}{16} a_{2}\right), \\
\eta^{*} & =\frac{1}{\nu_{1}^{*}-\frac{1}{2} \xi^{*}}, \\
\kappa^{*} & =\frac{1+2 a_{2}}{\nu_{2}^{*}-\frac{2 d}{d-1} \xi^{*}}, \\
\mu^{*} & =\frac{2 \kappa^{*} \xi^{*}+\frac{2(d-1)}{d} a_{2}}{\frac{2(d-1)}{d} \nu_{2}^{*}-3 \xi^{*}}, \\
\nu_{1}^{*} & =\frac{1}{4 d}(3-3 \varepsilon+2 d)(1+\varepsilon)\left(1-\frac{1}{32} a_{2}\right), \\
\nu_{2}^{*} & =\frac{1+\varepsilon}{2 d-2}\left[(d-1)+\frac{3}{8}(d+8)(1-\varepsilon)+\frac{1}{256}(4+5 d-3(4-d) \varepsilon) a_{2}\right],
\end{aligned}
$$




\begin{tabular}{|c|c|c|c|c|c|c|c|c|c|c|c|}
\hline \multicolumn{3}{|c|}{ 2D (viscoelastic) } & \multicolumn{3}{|c|}{ 2D (hybrid model) } & \multicolumn{3}{|c|}{ 3D (viscoelastic) } & \multicolumn{3}{|c|}{ 3D (hybrid model) } \\
\hline$\tilde{\eta}_{1}^{2 D}$ & $\approx$ & 0.234 & $\tilde{\eta}_{1}^{2 D}$ & $\approx$ & 0.242 & $\tilde{\eta}_{1}^{3 D}$ & $\approx$ & 0.483 & $\tilde{\eta}_{1}^{3 D}$ & $\approx$ & 0.422 \\
\hline$\tilde{\kappa}_{1}^{2} D$ & $\approx$ & -0.700 & $\tilde{\kappa}_{1}^{2 D}$ & $\approx$ & -0.242 & $\tilde{\kappa}_{1}^{3 D} D$ & $\approx$ & 0.393 & $\tilde{\kappa}_{1}^{3 D}$ & $\approx$ & 0.460 \\
\hline$\tilde{\mu}_{1}^{2 D}$ & $\approx$ & 1.811 & $\tilde{\mu}_{1}^{2} D$ & $\approx$ & 1.550 & $\tilde{\mu}_{1}^{3} D$ & $\approx$ & 1.229 & $\tilde{\mu}_{1}^{3} D$ & $\approx$ & 1.033 \\
\hline$\tilde{\xi}_{1}^{2 D} D$ & $\approx$ & 1.294 & $\tilde{\xi}_{1}^{2} D$ & $\approx$ & 1.033 & $\tilde{\xi}_{1}^{3} D$ & $\approx$ & 1.078 & $\tilde{\xi}_{1}^{3} D$ & $\approx$ & 0.861 \\
\hline$\tilde{\eta}_{2}^{2 D}$ & $\approx$ & 0.308 & $\tilde{\eta}_{2}^{2 D}$ & $\approx$ & 0.0763 & $\tilde{\eta}_{2}^{3 D} D$ & $\approx$ & 0.0942 & $\tilde{\eta}_{2}^{3 D} D$ & $\approx$ & 0.0323 \\
\hline$\tilde{\kappa}_{2}^{2 D}$ & $\approx$ & 11.89 & $\tilde{\kappa}_{2}^{2 D}$ & $\approx$ & 5.448 & $\tilde{\kappa}_{2}^{3 D}$ & $\approx$ & 4.904 & $\tilde{\kappa}_{2}^{3 D}$ & $\approx$ & 2.278 \\
\hline$\tilde{\mu}_{2}^{2} D$ & $\approx$ & 0.0562 & $\tilde{\mu}_{2}^{2} D$ & $\approx$ & -0.0958 & $\tilde{\mu}_{2}^{3} D$ & $\approx$ & 1.415 & $\tilde{\mu}_{2}^{3 D}$ & $\approx$ & 0.800 \\
\hline$\tilde{\xi}_{2}^{2 D}$ & $\approx$ & -2.093 & $\tilde{\xi}_{2}^{2 D}$ & $\approx$ & -1.304 & $\tilde{\xi}_{2}^{3 D} D$ & $\approx$ & -1.644 & $\tilde{\xi}_{2}^{3 D}$ & $\approx$ & -1.045 \\
\hline
\end{tabular}

Table 2.1: Coefficients for full viscoelastic model and the Taylor expansion of the static transport coefficients.

We can now use Eq. (2.48) together with Eq. (2.57), Eq. (2.59) and Eq. (2.60) to obtain the complete set of the hydrodynamic equations at NS level. Unless otherwise mentioned, we include only the first nonvanishing terms in the transport coefficients and do not include the bulk viscosity, which is a good approximation in the dilute granular gas case. The implementation of higher order corrections in our solver decreases the performance and therefore the focus on the first nonvanishing terms is a compromise between physical accuracy and performance. It has been shown that the contribution of higher order terms in the cooling factor can be neglected against the zeroth order term in dilute gases $[17,57]$. With these simplification we assume that this holds even in denser regimes.

The transport coefficients in Eq. (2.60) were first derived with the Chapman-Enskog approach $[17,57]$. Quite recently the same set of equations including the second Sonine coefficient have been derived with Grad's moment method [58] and a complete agreement in the results is shown. That both methods lead to the same NS equations gives confidence in the governing equation, which describe the hydrodynamic behavior of a granular gas.

The viscoelastic model in $2 \mathrm{D}$ and $3 \mathrm{D}$ for dilute gases (neglecting $\eta_{B}$ ) reads to the first and second order in the reduced time $\delta^{\prime}$ (in Eq. (2.12)) as

$$
\begin{aligned}
\eta_{2 D}^{*} & =1+\tilde{\eta}_{1}^{2 D} \delta^{\prime}+\tilde{\eta}_{2}^{2 D} \delta^{\prime 2}+\mathcal{O}\left(\delta^{\prime 3}\right) \\
\eta_{3 D}^{*} & =1+\tilde{\eta}_{1}^{3 D} \delta^{\prime}+\tilde{\eta}_{2}^{3 D} \delta^{\prime 2}+\mathcal{O}\left(\delta^{\prime 3}\right) \\
\kappa_{2 D}^{*} & =1+\tilde{\kappa}_{1}^{2 D} \delta^{\prime}+\tilde{\kappa}_{2}^{2 D} \delta^{\prime 2}+\mathcal{O}\left(\delta^{\prime 3}\right) \\
\kappa_{3 D}^{*} & =1+\tilde{\kappa}_{1}^{3 D} \delta^{\prime}+\tilde{\kappa}_{2}^{3 D} \delta^{\prime 2}+\mathcal{O}\left(\delta^{\prime 3}\right) \\
\mu_{2 D}^{*} & =\tilde{\mu}_{1}^{2 D} \delta^{\prime}+\tilde{\mu}_{2}^{2 D} \delta^{\prime 2}+\mathcal{O}\left(\delta^{\prime 3}\right) \\
\mu_{3 D}^{*} & =\tilde{\mu}_{1}^{3 D} \delta^{\prime}+\tilde{\mu}_{2}^{3 D} \delta^{\prime 2}+\mathcal{O}\left(\delta^{\prime 3}\right) \\
\xi_{2 D}^{*} & =\tilde{\xi}_{1}^{2 D} \delta^{\prime}+\tilde{\xi}_{2}^{2 D} \delta^{\prime 2}+\mathcal{O}\left(\delta^{\prime 3}\right) \\
\xi_{3 D}^{*} & =\tilde{\xi}_{1}^{3 D} \delta^{\prime}+\tilde{\xi}_{2}^{3 D} \delta^{\prime 2}+\mathcal{O}\left(\delta^{\prime 3}\right),
\end{aligned}
$$

where all constants are shown in Table 2.1. The full derivation of the moments of the collision integral which leads to the correct transport coefficients for viscoelastic particles in Eq.(2.61) can be found in [20, 30, 51, 57]. 


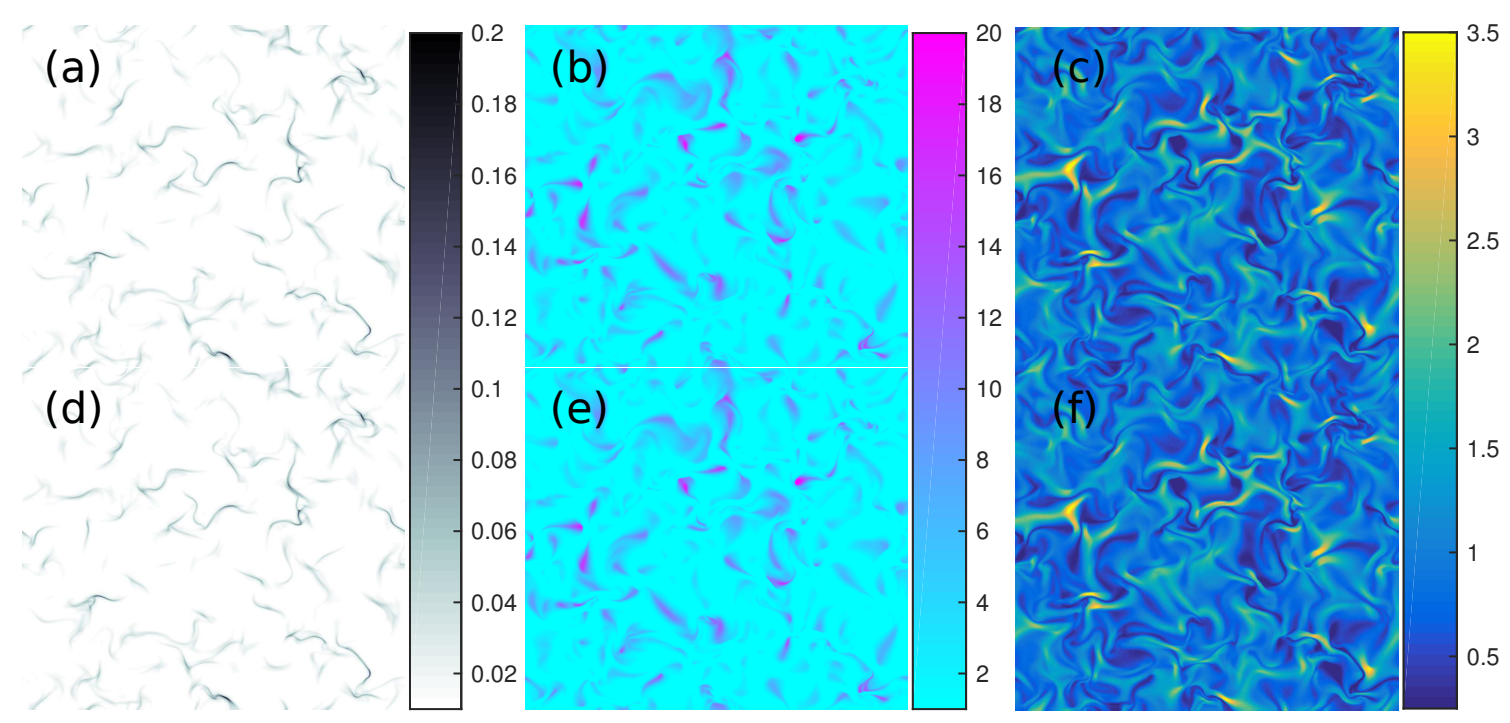

Figure 2.2: Comparison of the full viscoelastic model (top panels) and the hybrid model (bottom panels). The figure shows the filling fraction (a), (d), the local Mach number $\mathcal{M}=\frac{v}{\sqrt{T}}$ (b), (e) and the temperature (c), (f) in terms of the average temperature in the system. Both simulations started with the same random initial conditions and the snapshots are taken after a time $t=6000 \sigma / v_{t h, 0}$, where $v_{t h, 0}$ is the initial thermal velocity. The system parameters are: $L_{s y s}=51200 \sigma, \bar{\phi}=0.01, E(t=0)=0.95$. The initial configuration is a homogeneous cooling state.

We suggest another intuitive Ansatz for a viscoelastic model. If we assume that for a short moment in time $\varepsilon$ is nearly constant, then the transport coefficients in Eq. (2.60) hold and can be expanded around $\varepsilon$. The assumption is not too unrealistic because of the dependency of $\varepsilon \sim T^{1 / 10}$, which means that the temperature has to change drastically for it to affect $\varepsilon$. Then we can use Eq. (2.17) or (2.18) to derive the local coefficient of restitution to first or second order in $T\left(\delta^{\prime}\right)$ and use this one in Eq. (2.60). That means that we can perform a Taylor expansion of Eq. (2.60) around $\delta^{\prime}=0$. The parameter for this model obtained from Taylor expansion around constant $\varepsilon$ coefficients are shown in Table 2.1 and we name it "hybrid model", because we calculate the transport coefficients with the formula for a constant coefficient of restitution but use a temperature dependent $\varepsilon$ inside. In [30] it is mentioned that the kinetic coefficients for the constant and viscoelastic model differ significantly. We find that both models are in quite good numerical agreement with each other and show the same behavior of the transport coefficients. The viscoelastic model has the disadvantage that the series for the transport coefficients diverges for early times, which means for $T \approx T_{0}$. In this case the full viscoelastic model does not reproduce the results for a constant coefficient of restitution if $\varepsilon$ didn't changed significantly.

Figure 2.2 shows different physical observables of freely cooling granular gases (see Chapter 4) implementing both the hybrid and the viscoelastic model of $\varepsilon$ after $t=6000$. The systems started from the same initial state and shows the same qualitative behavior. It is hard by eye to observe a difference between the two models. Figure 2.3 is a plot of the differences in the density in Fig. 2.2. It is likely that because of small differences in 


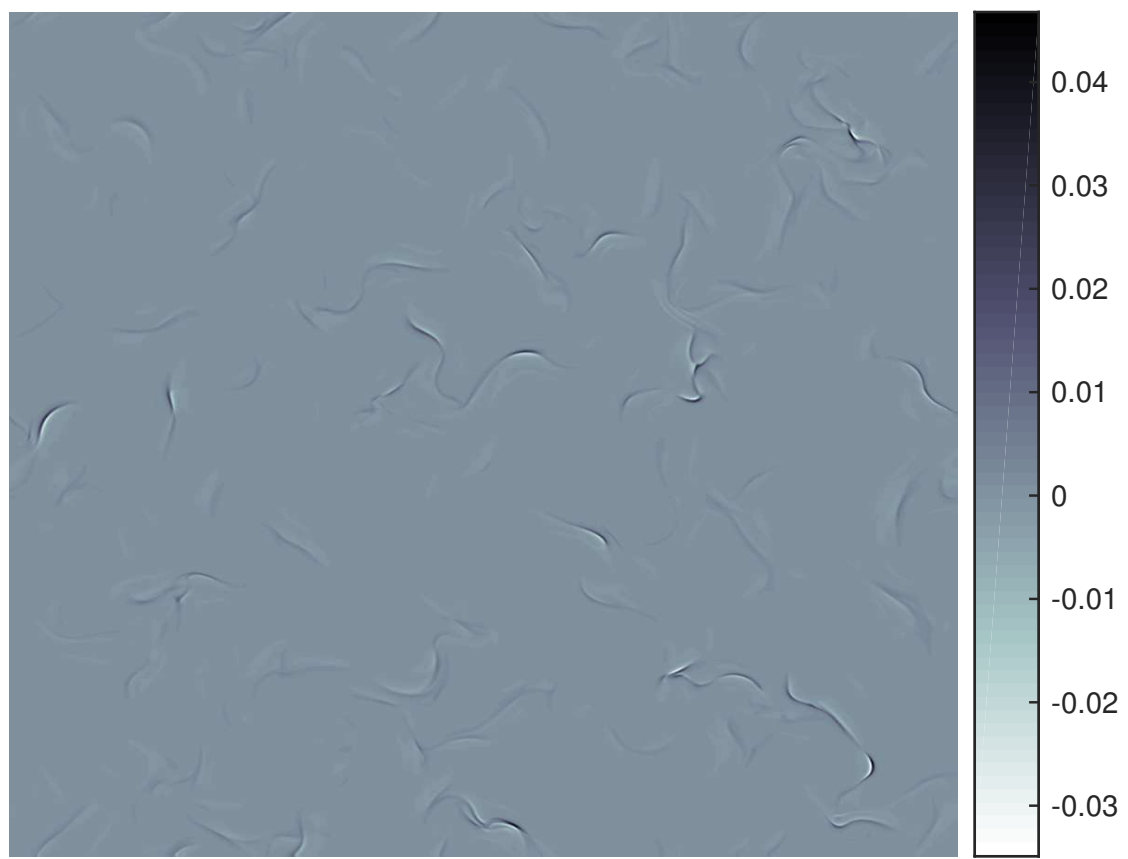

Figure 2.3: Difference of the filling fraction between the full viscoelastic model (Fig. 2.2(a)) and the hybrid model (Fig. 2.2(b)).

the convective velocities between both models, some of the clusters moved a bit and lead to an error in this figure. Nevertheless, Figs. 2.2 and 2.3 show that from our simulations we do not observe any qualitative nor quantitative difference between the two models. We can state out that the both models leads to similar results. Because of the superior performance within a numerical solver, we use the hybrid model inside our DNS.

\subsection{Haff's law for a free cooling granular gas in the homogeneous cooling state}

In 1983 Haff [19], who used the conservation laws of density, momentum and energy, gave an explicit solution of the evolution of the thermal velocity and the pressure in a homogeneous granular gas. Since then this analytical solution is known as Haff's law. Haff's law can be seen as the basis solution for the granular homogeneous gas. The linear stability analysis (see Section 2.9) proves that this state is instable because fluctuations in the velocity and density grow. Nevertheless here we assume a total initial homogeneous state, which means

$$
\begin{aligned}
\rho\left(\underline{\mathbf{r}}, t_{0}\right) & \equiv \rho_{0} \\
\underline{\mathbf{v}}\left(\underline{\mathbf{r}}, t_{0}\right) & \equiv \mathbf{0} \\
T\left(\underline{\mathbf{r}}, t_{0}\right) & \equiv T_{0} .
\end{aligned}
$$


The hydrodynamic equations in Eq. (2.48) simplify under the assumptions in Eq. (2.62) (all spacial gradients vanish) simply to

$$
\begin{aligned}
\partial_{t} \rho & =0 \\
\partial_{t} \underline{\mathbf{v}} & =\mathbf{0}, \\
\partial_{t} T & =-\xi_{0} \xi^{*} T .
\end{aligned}
$$

We obtain immediately $\rho(\underline{\mathbf{r}}, t)=\rho_{0}$ and $\underline{\mathbf{v}}(\underline{\mathbf{r}}, t)=\mathbf{0}$. In the temperature equation there appears only the zeroth order term of the cooling factor; higher order corrections vanish because of the vanishing spacial gradients. The cooling factor is known from Eqs. (2.59) and (2.60), then the equation can be easily solved by separation of variables

$$
\begin{aligned}
\partial_{t} T & =-\frac{\rho T}{\frac{2+d}{2^{d+1}}\left(\pi m_{p} T\right)^{1 / 2}\left(A_{p} g_{2}\right)^{-1}} \xi^{*} T \\
\int_{T_{0}}^{T} \frac{\mathrm{d} T^{\prime}}{T^{\prime 3 / 2}} & =-\int_{0}^{t} \frac{2^{d+1} \rho_{0} A_{p} g_{2}}{(2+d)\left(\pi m_{p}\right)^{1 / 2}} \xi^{*} \mathrm{~d} \tau^{\prime} \\
2\left(T_{0}^{-1 / 2}-T^{-1 / 2}\right) & =-t \tau^{-1} 2 T_{0}^{1 / 2} \\
T(t) & =\frac{T_{0}}{\left(1+t \tau^{-1}\right)^{2}}
\end{aligned}
$$

where $\tau$ is named the characteristic cooling time

$$
\tau^{-1}=\frac{2^{d} \rho_{0} A_{p} g_{2}}{(2+d)\left(\pi m_{p}\right)^{1 / 2}} \xi^{*} T_{0}^{1 / 2}
$$

We present here Haff's law in Eq. (2.64) for the temperature. For the model of viscoelastic spheres Haff's law looks slightly different [20]

$$
T(t)=\frac{T_{0}}{\left(1+t \tau^{-1}\right)^{5 / 3}},
$$

where it is important to stress that the slope changes, which is clear due to the fact that the granular gas becomes more and more elastic over time in the model of viscoelastic spheres. 


\subsection{Linear stability analysis of the homogeneous cooling state}

One of the most studied phenomena in granular gases is the spontaneous cluster formation out of a homogeneous cooling state (HCS) only due to the dissipative character of a granular gas. The emergence of the inhomogeneous cooling state (ICS) is in complete agreement with the predictions from linear stability analysis [20, 30, 34, 35]. A common argument to understand the formation of clusters starts with small fluctuation in the density of a homogeneous granular gas. In this denser region more collisions appear and lead to a spacial drop of the temperature. This leads to a sink of the pressure with respect to more dilute regions, which causes more particles to move inside the denser region, which is the seed of an amplifying process. We discuss this heuristic in Chapter 6 and we will see that we have evidence against this argumentation. Nevertheless, the linear stability analysis is untouched by the argumentation and is in perfect agreement with our observations in chapter 5 . We start from the homogeneous state where we assume only small fluctuations in the hydrodynamic fields

$$
\begin{aligned}
\rho(\boldsymbol{r}, t) & =\rho_{0}[1+\delta(\boldsymbol{r}, t)], \quad \delta \ll 1, \\
\boldsymbol{v}(\boldsymbol{r}, t) & =\sqrt{2 / m_{p}} T_{H}^{1 / 2}(t) \boldsymbol{\omega}(\boldsymbol{r}, t), \quad|\boldsymbol{\omega}| \ll 1, \\
T(\boldsymbol{r}, t) & =T_{H}(t)[1+\theta(\boldsymbol{r}, t)], \quad \theta \ll 1,
\end{aligned}
$$

where $\sqrt{2 T_{H}(t) / m_{p}}$ is the thermal velocity. The hydrodynamic equations (2.48) in terms of the primitive variables $\{\rho, \boldsymbol{v}, T\}$ and using the transport coefficients in Eq. (2.57) can be written as

$$
\begin{aligned}
& \partial_{t} \rho+\nabla \cdot(\rho \boldsymbol{v})=0, \\
& \frac{\mathrm{D}}{\mathrm{D} t} \boldsymbol{v}+\frac{1}{\rho m_{p}} \nabla \cdot\left(p \mathbf{1}-\eta\left(\nabla v+(\nabla v)^{T}-\frac{2}{d}(\nabla \cdot \boldsymbol{v}) \mathbf{1}\right)\right)=0, \\
& \frac{\mathrm{D}}{\mathrm{D} t} T+\frac{2}{d \rho}\left(p \mathbf{1}-\eta\left(\nabla v+(\nabla v)^{T}-\frac{2}{d}(\nabla \cdot \boldsymbol{v}) \mathbf{1}\right)\right): \nabla \boldsymbol{v}+\frac{2}{d \rho} \nabla \cdot(-\kappa \nabla T-\mu \nabla \rho)=-\xi_{0} \xi^{*} T,
\end{aligned}
$$

where we assume a dilute gas, which means the pressure is assumed to be the ideal gas pressure $p=\rho T$ and we neglect the bulk viscosity and higher order terms in the cooling state. The transport coefficients $\eta\left(T, g_{2}\right), \kappa\left(T, g_{2}\right), \mu\left(T, g_{2}\right)$ are taken at the basis state, which is given by Haff's law $T_{H}$ and a constant pair correlation function $g_{2}\left(\rho_{0}\right)$, otherwise the resulting equations would not be linear in $\{\delta, \boldsymbol{\omega}, \theta\}$. We assume the homogeneous cooling as the basis and expand Eq. (2.68) to linear order in $\delta, \omega, \theta$. The continuity equation becomes

$$
\partial_{t} \rho+\nabla \cdot(\rho \boldsymbol{v}) \approx \partial_{t}\left(\rho_{0} \delta\right)+\rho_{0} \nabla \cdot \boldsymbol{v}
$$




$$
\partial_{t} \delta+\left(2 T_{H} / m_{p}\right)^{1 / 2} \nabla \cdot \boldsymbol{\omega}=0
$$

For the time derivative of the velocity we obtain

$$
\begin{aligned}
\partial_{t} \boldsymbol{v} & =\partial_{t}\left[\left(2 T_{H} / m_{p}\right)^{1 / 2} \boldsymbol{\omega}\right]=\left(2 T_{H} / m_{p}\right)^{1 / 2} \partial_{t} \boldsymbol{\omega}+\boldsymbol{\omega} \partial_{t}\left[\left(2 T_{H} / m_{p}\right)^{1 / 2}\right] \\
& =\left(2 T_{H} / m_{p}\right)^{1 / 2} \partial_{t} \boldsymbol{\omega}+\frac{1}{2}\left[2 /\left(T_{H} m_{p}\right)\right]^{1 / 2} \boldsymbol{\omega} \partial_{t} T_{H} \\
& =\left(2 T_{H} / m_{p}\right)^{1 / 2} \partial_{t} \boldsymbol{\omega}-\frac{1}{\sqrt{2 m_{p}}} \boldsymbol{\omega} \xi_{0, H} \xi^{*} T_{H}^{1 / 2} \\
& =\left(2 T_{H} / m_{p}\right)^{1 / 2}\left(\partial_{t} \boldsymbol{\omega}-\frac{1}{2} \boldsymbol{\omega} \xi_{0, H} \xi^{*}\right)
\end{aligned}
$$

where $\xi_{0, H}$ is the homogeneous cooling coefficient, computed with $\xi_{0}$ by $\rho \rightarrow \rho_{0}$ and $T \rightarrow T_{H} ; \partial_{t} T_{H}$ follows from Haff's law Eq. (2.64) (see Section 2.8). Linearizing the pressure gradient leads to

$$
\frac{1}{\rho m_{p}} \nabla p=\frac{1}{m_{p}} T_{H} \nabla \delta+\frac{1}{m_{p}} T_{H} \nabla \theta .
$$

We neglect the nonlinear term $\boldsymbol{v} \cdot \nabla \boldsymbol{v}$ in the velocity equation and obtain

$$
\begin{aligned}
& \sqrt{2 / m_{p}} T_{H}^{1 / 2} \partial_{t} \boldsymbol{\omega}-\frac{1}{\sqrt{2 m_{p}}} \boldsymbol{\omega} \xi_{0} \xi^{*} T_{H}^{1 / 2}+\frac{T_{H}}{m_{p}}(\nabla \delta+\nabla \theta) \\
& =\sqrt{2 / m_{p}} T_{H}^{1 / 2} \frac{\eta}{\rho_{0} m_{p}} \nabla \cdot\left(\nabla \boldsymbol{\omega}+(\nabla \boldsymbol{\omega})^{T}-\frac{2}{d}(\nabla \cdot \boldsymbol{\omega}) \mathbf{1}\right) .
\end{aligned}
$$

The linearized form of the time derivative of the temperature is

$$
\begin{aligned}
\partial_{t} T & =\partial_{t}\left(T_{H}(1+\theta)\right) \\
& \approx(1+\theta) \partial_{t} T_{H}+T_{H} \partial_{t} \theta \\
& =-(1+\theta) \xi^{*} C_{\beta}\left(2 m_{p}\right)^{-1 / 2} T_{H}^{3 / 2}+T_{H} \partial_{t} \theta
\end{aligned}
$$

where we have used the relation

$$
\partial_{t} T_{H}=-\xi_{0, H} \xi^{*} T_{H}=-\xi^{*} C_{\beta}\left(2 m_{p}\right)^{-1 / 2} T_{H}^{3 / 2}
$$

with the constant $C_{\beta}=\frac{2^{d+1}\left(A_{p} \rho_{0} g_{2}\right)}{(2+d)(\pi / 2)^{1 / 2}}=\frac{\tilde{C}_{\beta}}{\sigma}$ and $\tilde{C}_{\beta}=\frac{2^{d+2} d}{(2+d)(\pi / 2)^{1 / 2}} g_{2} \phi_{0}$.

The cooling term up to linear order leads to

$$
\begin{aligned}
-\xi_{0} \xi^{*} T & =-\xi^{*} \frac{\rho T}{\eta_{0}} T \\
& =-\xi^{*} \frac{\rho T}{\frac{\rho_{0}}{C_{\beta}}\left(2 m_{p} T\right)^{1 / 2}} T \\
& =-\xi^{*} C_{\beta}\left(2 m_{p}\right)^{-1 / 2}\left((1+\delta) T_{H}^{3 / 2}(1+\theta)^{3 / 2}\right) \\
& \simeq-\xi^{*} C_{\beta}\left(2 m_{p}\right)^{-1 / 2} T_{H}^{3 / 2}\left(1+\delta+\frac{3}{2} \theta\right)
\end{aligned}
$$


where we assume that the filling fraction is small enough to neglect the term $\partial_{\rho} g_{2}$.

The last step is the linearized form of the derivative of the heat flux

$$
\begin{aligned}
\nabla \cdot \boldsymbol{q} & =-\nabla \cdot(\kappa \nabla T+\mu \nabla \rho) \\
& \approx-\left(\kappa_{0, H} \kappa^{*} T_{H} \nabla^{2} \theta+\mu_{0, H} \mu^{*} \rho_{0} \nabla^{2} \delta\right), \\
& =-\frac{d(d+2)}{2(d-1)} \frac{\rho_{0}}{C_{\beta}}\left(2 T_{H} / m_{p}\right)^{1 / 2}\left(\kappa^{*} T_{H} \nabla^{2} \theta+\frac{T_{H}}{\rho_{0}} \mu^{*} \rho_{0} \nabla^{2} \delta\right) \\
& =-\frac{d(d+2)}{2(d-1)} \frac{1}{C_{\beta}}\left(2 / m_{p}\right)^{1 / 2} \rho_{0} T_{H}^{3 / 2}\left(\kappa^{*} \nabla^{2} \theta+\mu^{*} \nabla^{2} \delta\right) .
\end{aligned}
$$

We thus obtain the linearized equation for the temperature fluctuations

$$
\begin{aligned}
T_{H} \partial_{t} \theta & +\frac{2}{d}\left(2 / m_{p}\right)^{1 / 2} T_{H}^{3 / 2} \nabla \cdot \boldsymbol{\omega}-\frac{d+2}{d-1} \frac{1}{C_{\beta}}\left(2 / m_{p}\right)^{1 / 2} T_{H}^{3 / 2}\left(\kappa^{*} \nabla^{2} \theta+\mu^{*} \nabla^{2} \delta\right) \\
& +\xi^{*} C_{\beta}\left(2 m_{p}\right)^{-1 / 2} T_{H}^{3 / 2}\left(\delta+\frac{1}{2} \theta\right)=0 .
\end{aligned}
$$

Further simplification leads to the final set of linearized equations

$$
\begin{aligned}
\left(\frac{m_{p}}{2 T_{H}}\right)^{1 / 2} \partial_{t} \delta+\nabla \cdot \boldsymbol{\omega} & =0, \\
\left(\frac{m_{p}}{2 T_{H}}\right)^{1 / 2} \partial_{t} \boldsymbol{\omega}-\frac{1}{4} C_{\beta} \xi^{*} \boldsymbol{\omega}+\frac{1}{2}(\nabla \delta+\nabla \theta)-\frac{1}{C_{\beta}} \eta^{*}\left(\nabla^{2} \omega+\frac{d-2}{d} \nabla(\nabla \cdot \boldsymbol{\omega})\right) & =0, \\
\left(\frac{m_{p}}{2 T_{H}}\right)^{1 / 2} \partial_{t} \theta+\frac{2}{d} \nabla \cdot \boldsymbol{\omega}-\frac{1}{C_{\beta}} \frac{d+2}{d-1}\left(\kappa^{*} \nabla^{2} \theta+\mu^{*} \nabla^{2} \delta\right)+C_{\beta} \xi^{*}\left(\frac{1}{2} \delta+\frac{1}{4} \theta\right) & =0 .
\end{aligned}
$$

We assume a volume of $\left(L_{\text {sys }}\right)^{d}$ and perform the Fourier transform of the hydrodynamic fields, where we use the definition of the Fourier transform for a field $\boldsymbol{a}$

$$
\begin{aligned}
\boldsymbol{a}_{\boldsymbol{k}}(t) & =L_{\text {sys }}^{-d / 2} \int \mathrm{e}^{-\mathrm{i} \boldsymbol{k} \boldsymbol{r}} \boldsymbol{a}(\boldsymbol{r}, t) \mathrm{d} \boldsymbol{r} \\
\boldsymbol{a}(\boldsymbol{r}, t) & =L_{\text {sys }}^{-d / 2} \sum_{\boldsymbol{k}} \mathrm{e}^{\mathrm{i} \boldsymbol{k} \boldsymbol{r}} \boldsymbol{a}_{\boldsymbol{k}}(t) .
\end{aligned}
$$

The summation runs over all modes $\boldsymbol{k}$

$$
\boldsymbol{k}=\frac{2 \pi}{L_{\text {sys }}}\left(\begin{array}{l}
n_{x} \\
n_{y} \\
n_{z}
\end{array}\right) \quad n_{x}, n_{y}, n_{z}=\{0, \pm 1, \pm 2, \ldots\}
$$


By using the Fourier transform, the equations (2.78) become

$$
\begin{aligned}
& \left(\frac{m_{p}}{2 T_{H}}\right)^{1 / 2} \partial_{t} \delta_{\boldsymbol{k}}=-\mathrm{i} \boldsymbol{k} \cdot \boldsymbol{\omega}_{\boldsymbol{k}} \\
& \left(\frac{m_{p}}{2 T_{H}}\right)^{1 / 2} \partial_{t} \boldsymbol{\omega}_{\boldsymbol{k}}=\frac{1}{4} C_{\beta} \xi^{*} \boldsymbol{\omega}_{\boldsymbol{k}}-\mathrm{i} \boldsymbol{k} \frac{1}{2}\left(\delta_{\boldsymbol{k}}+\theta_{\boldsymbol{k}}\right)-\frac{1}{C_{\beta}} \eta^{*}\left(\boldsymbol{k}^{2} \omega_{\boldsymbol{k}}+\frac{d-2}{d} \boldsymbol{k}\left(\boldsymbol{k} \cdot \boldsymbol{\omega}_{\boldsymbol{k}}\right)\right) \\
& \left(\frac{m_{p}}{2 T_{H}}\right)^{1 / 2} \partial_{t} \theta_{\boldsymbol{k}}=-\frac{2}{d}\left(\mathrm{i} \boldsymbol{k} \cdot \boldsymbol{\omega}_{\boldsymbol{k}}\right)-\frac{1}{C_{\beta}} \frac{2+d}{d-1}\left(\kappa^{*} \boldsymbol{k}^{2} \theta_{\boldsymbol{k}}+\mu^{*} \boldsymbol{k}^{2} \delta_{\boldsymbol{k}}\right)-C_{\beta} \xi^{*}\left(\frac{1}{2} \delta_{\boldsymbol{k}}+\frac{1}{4} \theta_{\boldsymbol{k}}\right) .
\end{aligned}
$$

It is usefully to change the time variable

$$
\alpha(t)=\left(\frac{2 T_{H}}{m_{p}}\right)^{1 / 2} \tau \ln \left(1+t \tau^{-1}\right),
$$

where $\tau$ is the cooling time in Eq. (2.65). With this transformation we can write Eq. (2.81) in matrix form

$$
\partial_{\alpha} \Psi=\underline{\mathbf{M}}_{F} \boldsymbol{\Psi}
$$

where $\underline{\mathbf{M}}_{F}$ is a $(2+d) \times(2+d)$ matrix. The eigenvectors of $\underline{\mathbf{M}}_{F}$, which are defined by

$$
\underline{\mathbf{M}}_{F} \boldsymbol{\Psi}_{n}=\lambda_{n} \boldsymbol{\Psi}_{n}, \quad n=1, \ldots, 2+d,
$$

evolve independently

$$
\Psi_{n}(\alpha)=\boldsymbol{\Psi}(0) \mathrm{e}^{\lambda_{n} \alpha}
$$

The $\boldsymbol{\Psi}_{n}$ are called the hydrodynamic modes. Given the direction of the wavevector $\boldsymbol{k}$, we can decompose the convective velocity fluctuation $\boldsymbol{\omega}$ into a longitudinal mode $\boldsymbol{\omega}_{\boldsymbol{k}, \|} \equiv$ $\left(\boldsymbol{\omega}_{\boldsymbol{k}} \cdot \boldsymbol{k}\right) \frac{\boldsymbol{k}}{k^{2}}$ and a transversal mode $\boldsymbol{\omega}_{\boldsymbol{k}, \perp} \equiv \boldsymbol{\omega}_{\boldsymbol{k}}-\boldsymbol{\omega}_{\boldsymbol{k}, \|}$. Using $\boldsymbol{k} \cdot \boldsymbol{\omega}_{\boldsymbol{k}, \perp}=0$, we can decompose the second equation of Eq. (2.81) into two equations for the transversal and one for the longitudinal mode. The resulting equation for the transversal mode

$$
\partial_{\alpha} \omega_{k, \perp}=\left(\frac{1}{4} \xi^{*}-\eta^{*} k^{2}\right) \omega_{k, \perp}
$$

decouples from the other equations, and has two degenerate eigenvalues $\lambda_{\perp, 1}=\lambda_{\perp, 2}=$ $\frac{1}{4} \xi^{*}-\eta^{*} k^{2}$, which causes a shear instability if the system exceeds a certain size (more on this topic can be found in the literature e.g. in [30]). The reduced $3 \times 3$ matrix $\underline{\mathbf{M}}$ reads as

$$
\underline{\mathbf{M}}=\left(\begin{array}{ccc}
0 & -\mathrm{i} k & 0 \\
\frac{-\mathrm{i} k}{2} & \frac{1}{4} C_{\beta} \xi^{*}-2 \frac{d-1}{d} \frac{1}{C_{\beta}} \eta^{*} k^{2} & \frac{-\mathrm{i} k}{2} \\
-\frac{1}{C_{\beta}} \frac{2+d}{d-1} \mu^{*} k^{2}-\frac{1}{2} C_{\beta} \xi^{*} & -\frac{2}{d} \mathrm{i} k & -\frac{1}{C_{\beta}} \frac{2+d}{d-1} \kappa^{*} k^{2}-\frac{1}{4} C_{\beta} \xi^{*}
\end{array}\right),
$$




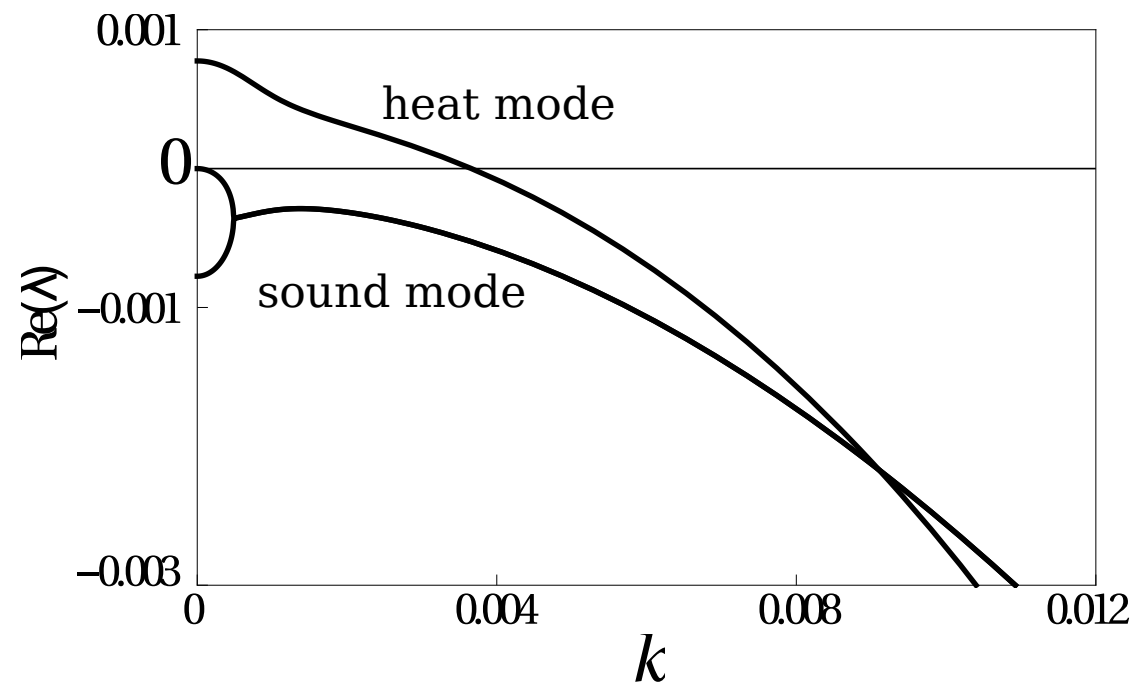

Figure 2.4: Real part of the eigenvalues of the reduced system in Eq. (2.87). The figure shows the result for $\varepsilon=0.95$ and $\bar{\phi}=0.01$.

which contains now only the longitudinal mode $\omega_{\|}$in the second row. The eigenvalues of $\underline{\mathbf{M}}$ are rather cumbersome and can be calculated with a computer algebraic program like MAthematica ${ }^{\circledR}$. In the Appendix 10.1, we give the eigenvalues and the corresponding eigenvectors of $\underline{\mathbf{M}}$.

Figure 2.4 shows the "heat" and the "sound" mode of the system in Eq. 2.87. Importantly, only the heat mode has a real positive eigenvalue, $\operatorname{Re}(\lambda)>0$. This positive eigenvalue corresponds to an exponential growth of the instabilities. This produces the density instability. The root $\operatorname{Re}(\lambda(k))=0$ defines the critical wavenumber $k_{H}^{*}$. Because this root is the only root we find for any $\lambda(k)$, we obtain it by finding the root of the determinant of $\underline{\mathbf{M}}$.

$$
\begin{aligned}
\operatorname{det}|\underline{\mathbf{M}}| & \left.=-(2+d) k^{4}\left(\kappa^{*}-\mu^{*}\right)\right) /\left(2 C_{\beta}(d-1)\right)+1 / 8 C_{\beta} k^{2} \xi^{*}=0 \\
k_{H}^{*}(\varepsilon) & =\frac{C_{\beta}}{2}\left(\frac{\xi^{*}}{\frac{2+d}{d-1}\left(\kappa^{*}-\mu^{*}\right)}\right)^{1 / 2} \propto \rho_{0}\left(1-\varepsilon^{2}\right)^{1 / 2}
\end{aligned}
$$

We can express the wavenumber in terms of $\frac{1}{\sigma}$ by replacing $C_{\beta}$ with $\frac{\tilde{C}_{\beta}}{\sigma}$. This result indicates that we find clustering only in systems where the system size $L$ exceeds the critical system size

$$
L_{\text {crit }}=2 \pi / k_{H}^{*}(\varepsilon) \text {. }
$$

In Chapter 7 we will have a closer look at finite size effects. Our results in Section 7.2 are in complete agreement with the theoretical prediction from the linear stability analysis. As an example we can think of a 3D system containing particles of $1 \mathrm{~mm}$ size and $\bar{\phi}=0.01$ and $\varepsilon=0.8$, which can be realized in an experimental setup. We expect to see the free cooling clustering in this systems only if its linear size is larger then $0.5 \mathrm{~m}$. 


\section{Navier-Stokes equations of granular gases}

In this chapter we focus on the NS approach to hydrodynamics equation. We discuss in the beginning the pair correlation functions which we use in our approach and introduce Luding's pair correlation function in 2D to obtain even more realistic values at all densities. We present the dimensionless form of the equations and discuss the important dimensionless parameters Prandtl $\mathcal{P}$, Reynolds $\mathcal{R}$ and the Mach $\mathcal{M}$ number. We discuss the differences of these dimensionless numbers of granular gases from molecular fluids.

\subsection{Pair correlation function}

In hydrodynamic systems which contain finite-size particles we observe different states due to excluded volume effects, especially the transitions between the gas, liquid and solid state. In molecular gases this effect results in a correction to the ideal gas equation of state $p=\rho T f(\rho)$. The Boltzmann-Enskog equation (Eq. (2.38)) includes these effects via the pair-correlation function, which leads to a modification of the equation of state and corrections in the transport coefficients. The question arises what is the functional form of the pair-correlation function for granular gases. From MD simulations it is possible to calculate the correct pair-correlation function numerically. This gives the possibility of a comparison with a functional form. In a fully elastic system the Carnahan-Starling [59] pair correlation function gives very good results in a dilute regime

$$
g_{C S}(\sigma)=\frac{1-A(d) \phi}{(1-\phi)^{d}}
$$


with the filling fraction

$$
\phi=B(d) \pi \rho \sigma^{d}
$$

and $A(2)=\frac{7}{16}, A(3)=\frac{1}{2}, B(2)=\frac{1}{4}, B(3)=\frac{1}{6}$ are dimension-dependent constants. This pair correlation function is accurate for packing fractions smaller than 0.5 and thereby valid till medium densities (in 3D). It was shown that this is also true for dissipative gases [21]. The Carnahan-Starling pair correlation function leads, however, to wrong results at high densities; it diverges at $\phi=1$ and does not contain the maximum packing fraction. In addition Eq. (3.1) does not contain a transition point, which means that it will lead to the wrong pressure where we would expect a liquid-gas phase transition. To include transition effects to our hydrodynamic model we have to find a pair correlation function which leads to the correct physics for as large densities as possible. In $2 \mathrm{D}$, for higher densities a higher order term can be added to correct $g_{C S}[60]$

$$
g_{4}=\frac{1-7 / 16 \phi}{(1-\phi)^{2}}-\frac{\phi^{3}}{128(1-\phi)^{4}}
$$

If the packing fraction is close to the maximum packing fraction a good approximation is given by [60]

$$
g_{\text {dense }}=\frac{\phi_{m}}{\phi}\left(\left(\phi_{m}-\phi\right)^{-1}+c_{1}+c_{3}\left(\phi_{m}-\phi\right)^{2}-\frac{1}{2 \phi_{m}}\right) \text {, }
$$

with the maximum packing fraction $\phi_{m} \equiv \frac{\pi}{2 \sqrt{3}}$, the transition point $\phi_{c}=0.6990$ and fitting parameters $c_{1}=-0.04, c_{3}=3.25, \alpha_{\phi}=0.0111$. The combination of the pair correlation functions in Eq. (3.3) and Eq. (3.4) leads to

$$
g_{Q}=g_{4}+n(\phi)\left(g_{\text {dense }}-g_{4}\right)
$$

where

$$
n(\phi)=\left[1+\exp \left(\frac{\phi_{c}-\phi}{\alpha_{\phi}}\right)\right]^{-1} .
$$

The 2D pair correlation function in Eq. (3.5) was suggested by Luding [61]. The fitting parameter $\phi_{c}$ marks the liquid-solid transition in two dimension. The pair correlation function $g_{Q}$ is valid for all densities in $2 \mathrm{D}$ and leads to a global equation of state which deviates not more than $1 \%$ from results of MD simulations [60,61]. The maximum deviation appears during the transition regime at $\phi \approx \phi_{c}$; apart from this transient regime the error margin is smaller than $0.1 \%$. In addition Luding's pair correlation function leads to plausible results in all transport coefficients at all filling fractions [61]. We implemented the Luding's pair correlation function $g_{Q}$ in $2 \mathrm{D}$ and we use the Carnahan-Starling pair correlation function in 3D. 


\subsection{Quasi conservative form of the Navier-Stokes equations}

The full hydrodynamic equations including sources and external forces for a granular gas in $d$ dimensions read as

$$
\begin{aligned}
& \partial_{t} \rho+\nabla \cdot(\rho \boldsymbol{v})=0 \\
& \frac{\mathrm{D}}{\mathrm{D} t} \boldsymbol{v}+\frac{1}{\rho m_{p}} \nabla \cdot\left\{p \mathbb{1}-\eta\left[\nabla \boldsymbol{v}+(\nabla \boldsymbol{v})^{\mathrm{T}}-\left(\frac{2}{d} \nabla \cdot \boldsymbol{v}\right) \mathbb{1}\right]\right\}=\boldsymbol{a} \\
& \frac{\mathrm{D}}{\mathrm{D} t} T-\frac{2}{d \rho} \eta\left\{\left[\nabla \boldsymbol{v}+(\nabla \boldsymbol{v})^{\mathrm{T}}-\left(\frac{2}{d} \nabla \cdot \boldsymbol{v}\right) \mathbb{1}\right]: \nabla \boldsymbol{v}\right\}+ \\
& \quad \frac{2}{d \rho} p \nabla \cdot \boldsymbol{v}+\frac{2}{d \rho} \nabla \cdot \boldsymbol{q}=-\xi T
\end{aligned}
$$

where $\mathrm{D} / \mathrm{D} t \equiv \partial_{t}+(\boldsymbol{v} \cdot \nabla)$ is the material derivative and $\mathbb{1}$ is the unit tensor; in Eq. (3.6)(3.8) the hydrostatic pressure $p$ and the heat flux $\boldsymbol{q}$ are given by

$$
\begin{aligned}
& p=\rho T\left[1+(1+\varepsilon) 2^{d-2} \phi g_{2}(\rho)\right], \\
& \boldsymbol{q}=-\kappa \nabla T-\mu \nabla \rho,
\end{aligned}
$$

where $\phi$ is the filling fraction in Eq. (3.2) and $g_{2}(\rho)$ is the pair correlation function. The transport coefficients are defined in Eq. (2.58)

$$
\begin{aligned}
\eta & =\eta_{0} \eta^{*} \\
\eta_{B} & =\eta_{0} \eta_{B}^{*} \\
\kappa & =\kappa_{0} \kappa^{*} \\
\mu & =\mu_{0} \mu^{*} \\
\xi & =\xi_{0} \xi^{*},
\end{aligned}
$$

with

$$
\begin{aligned}
\eta_{0} & =\frac{2+d}{2^{d+1}} \sqrt{\pi m_{p} T}\left(A_{p} g_{2}\right)^{-1} \\
\kappa_{0} & =\frac{d(d+2)^{2}}{2^{2+d}(d-1)} \sqrt{\frac{\pi T}{m_{p}}}\left(A_{p} g_{2}\right)^{-1} \\
\mu_{0} & =\frac{\kappa_{0} T}{\rho} \\
\xi_{0} & =\frac{\rho T}{\eta_{0}}
\end{aligned}
$$

where $A_{p}=2(\sigma / 2)^{d-1} \frac{\pi^{d / 2}}{\Gamma(d / 2)}$ is the surface of a sphere in $d$ dimension; $\Gamma(x)$ is the Euler gamma function. The details of the dimensionless parameter $\eta^{*}, \eta_{B}^{*}, \kappa^{*}, \mu^{*}, \xi^{*}$ depend strongly on the underlying collision model and are described in Section 2.1.

The primitive variables density $\rho$, velocity $\boldsymbol{v}$ and temperature $T$, which appear in Eq. (3.6)-(3.8) are native variables which can be directly measured in most systems like 
molecular gases and fluids. In contrast to the primitive variables we call the variables number density $\rho$, momentum density $\rho \boldsymbol{v}$ and energy density $\rho E=\rho\left(\frac{1}{2} m_{p} \boldsymbol{v}^{2}+\frac{d}{2} T\right)$ the set of conservative variables $\boldsymbol{U}$. Conservative variables are typically not directly measurable (apart from the density), but these variables fulfill important conservation laws, namely: (i) mass continuity; (ii) conservation of momentum; (iii) conservation of energy. Therefore it makes sense to rewrite the set of NS equation to a quasi-conservative form. It is called quasi-conservative form, because the sources/forces acting on the system violate the conservation of the quantities and lead to extra terms in the conservation laws.

When rewriting the equations it is obvious that the density equation stays the same in primitive and conservative form. The momentum and energy density equations are straightforward to derive by using the product rule

$$
\begin{aligned}
& \partial_{t}(\rho \boldsymbol{v})-\boldsymbol{v} \partial_{t} \rho-\rho \partial_{t} \boldsymbol{v}=0 \\
& \partial_{t}(\rho E)-\left(\frac{1}{2} m_{p} \boldsymbol{v}^{2}+\frac{d}{2} T\right) \partial_{t} \rho-\rho m_{p} \boldsymbol{v} \partial_{t} \boldsymbol{v}-\rho \frac{d}{2} \partial_{t} T=0 .
\end{aligned}
$$

The time derivative of the primitive variables are known from the NS equation in primitive form (see Eq. (3.6)-(3.8)). The hydrodynamic equations in quasi-conservative form then read

$$
\begin{gathered}
\partial_{t} \rho+\nabla \cdot(\rho \boldsymbol{v})=0 \\
\partial_{t}(\rho \boldsymbol{v})+\nabla \cdot\left[\boldsymbol{v} \rho \boldsymbol{v}^{T}+\frac{1}{m_{P}} p \mathbb{1}\right]-\frac{1}{m_{P}} \nabla \cdot\left[\eta\left(\nabla \boldsymbol{v}+(\nabla \boldsymbol{v})^{T}-\frac{2}{d}(\nabla \cdot \boldsymbol{v}) \mathbb{1}\right)\right]=\rho \boldsymbol{a} \\
\partial_{t}(\rho E)+\nabla \cdot(\rho H \boldsymbol{v})-\nabla \cdot\left(\eta\left[\nabla \boldsymbol{v}+(\nabla \boldsymbol{v})^{T}-\frac{2}{d}(\nabla \cdot \boldsymbol{v}) \mathbb{1}\right] \cdot \boldsymbol{v}-\boldsymbol{q}\right) \\
=m_{P} \rho \boldsymbol{v} \cdot \boldsymbol{a}-\frac{d}{2} \xi \rho T
\end{gathered}
$$

where $H=E+\frac{p}{\rho}$ is the enthalpy.

\subsection{Dimensionless form of the Navier-Stokes equations}

It is often extremely useful to evaluate the ratio of two forces, or energies to determine the dominant physical regime. This knowledge leads to a simplification of the problem. In hydrodynamics, two of the most common dimensionless quantities are the Reynolds number $\mathcal{R} \equiv \frac{\text { inertial force }}{\text { viscous force }}=\frac{\rho v L}{\eta}$ and the Prandtl number $\mathcal{P} \equiv \frac{\text { viscous diffusion }}{\text { thermal diffusion }}=\frac{\eta / \rho}{\kappa /\left(\rho c_{p}\right)}$. A second, important reason to define dimensionless numbers is the concept of similitude of the dynamics in different physical systems. Two systems with different numbers of particles, or different viscosities, or even densities, may nonetheless have the same value of Reynolds number. This coincidence has as consequence that the dynamical behavior will be similar ${ }^{1}$. This means that two systems of different size and containing different

\footnotetext{
${ }^{1}$ In fluids dynamics an additional, stronger requirement is that the system have also similar geometrical shapes, when studying the motion of solid objects in a fluid
} 
particles behave similarly when they have the same intrinsic numbers. To obtain this dimensionless numbers, it is necessary to rewrite the equations in a dimensionless form.

Within this section we distinguish variables containing physical units $\hat{\alpha}$ from dimensionless variables $\alpha$ by using the hat sign. Both variables are connected via a reference number $\alpha_{r}$ which contains the characteristic units of the variable, e.g., the system size is a characteristic length in the system

$$
\hat{\alpha}=\alpha_{r} \alpha
$$

The reference numbers contains also the dimension of the variable, that means that all variables become of order of unity. This has the computational advantage that it leads often to more numerically robust equations. We describe below a set of reference variables to rewrite the equations. The reference length is $x_{r}$, the typical convective velocities is $v_{r}$, the density reference is $\rho_{r}=\bar{\phi} V_{p}^{-1}$, where $\bar{\phi}$ is the average filling fraction. That means that we measure the density in units of the average filling fraction. The temperature is measured in terms of the thermal velocity $\hat{T}=m_{p} \hat{v}_{T}^{2}$. Therefore a characteristic temperature

$$
T_{r}=m_{p} v_{T, r}^{2}
$$

must be derived from a characteristic thermal velocity $v_{T, r}$. The energy is similarly measured in terms of the characteristic temperature

$$
E_{r}=H_{r}=T_{r}=m_{p} v_{T, r}^{2} .
$$

Then the energy is calculated as

$$
E=\frac{1}{2} \mathcal{M}^{-2} \boldsymbol{v}^{2}+\frac{d}{2} T
$$

where

$$
\mathcal{M} \equiv \frac{v_{r}}{v_{T, r}}
$$

is the characteristic Mach number of the system. As in ordinary gases we define the Mach number as the ratio between convective and thermal velocities. We discuss the Mach number in more detail in Section 3.4. Forces lead to a characteristic acceleration $a_{r}$ in the system (e.g. gravitational acceleration). Out of this set of characteristic units we derive the following reference values for time and pressure

$$
\begin{aligned}
t_{r} & =x_{r} / v_{r}, \\
p_{r} & =\rho_{r} m_{p} v_{T, r}^{2} .
\end{aligned}
$$


Now we can rewrite the NS equations in (3.17) in a dimensionless form

$$
\begin{aligned}
& \partial_{t} \rho+\nabla \cdot(\rho \boldsymbol{v})=0 \\
& \partial_{t}(\rho \boldsymbol{v})+\nabla \cdot\left[\boldsymbol{v} \rho \boldsymbol{v}^{T}+\mathcal{M}^{-2} p \mathbb{1}\right]-\nabla \cdot\left[\frac{1}{\mathcal{R}}\left(\nabla \boldsymbol{v}+(\nabla \boldsymbol{v})^{T}-\frac{2}{d}(\nabla \cdot \boldsymbol{v}) \mathbb{1}\right)\right]=\frac{1}{\mathcal{F}} \rho \boldsymbol{a} \\
& \partial_{t}(\rho E)+\nabla \cdot(\rho H \boldsymbol{v})-\nabla \cdot\left(\frac{1}{\mathcal{R}}\left\{\mathcal{M}^{2}\left[\nabla \boldsymbol{v}+(\nabla \boldsymbol{v})^{T}-\frac{2}{d}(\nabla \cdot \boldsymbol{v}) \mathbb{1}\right] \cdot \boldsymbol{v}-\frac{1}{\mathcal{P}} \boldsymbol{q}\right\}\right) \\
& =\frac{1}{\mathcal{F}} \mathcal{M}^{2} \rho \boldsymbol{v} \cdot \boldsymbol{a}-\frac{d}{2} \mathcal{X} \rho T
\end{aligned}
$$

with the dimensionless pressure, heat flux and enthalpy

$$
\begin{aligned}
p & =\rho T\left[1+(1+\varepsilon) 2^{d-2} \bar{\phi} \rho g_{2}(\hat{\rho})\right], \\
\boldsymbol{q} & =-\left(\nabla T+\mathcal{C}_{\mu} \nabla \rho\right) \\
H & =E+\frac{p}{\rho} .
\end{aligned}
$$

The dimensionless variables

$$
\begin{aligned}
\mathcal{R} & =\frac{m_{p} \rho_{r} v_{r} x_{r}}{\hat{\eta}}=\frac{A_{P} \bar{\phi} \sqrt{d} x_{r} \mathcal{M}}{V_{P} \eta}=2 d^{3 / 2} \frac{x_{r}}{\sigma} \bar{\phi} \eta^{-1} \\
\mathcal{P} & =\frac{\hat{\eta}}{m_{p} \hat{\kappa}}=\frac{\eta}{\kappa} \\
\mathcal{F} & =\frac{v_{r}^{2}}{a_{r} x_{r}} \\
\mathcal{C}_{\mu} & =\frac{\rho_{r} \hat{\mu}}{T_{r} \hat{\kappa}}=\frac{\mu^{*}}{\kappa^{*}} \frac{T}{\rho} \\
\mathcal{X} & =\frac{\hat{\xi} x_{r}}{v_{r}}=\frac{\rho_{r} T_{r} x_{r}}{v_{r} m_{p} A_{P}^{-1} d^{-1 / 2} v_{T, r}} \xi^{*} \frac{\rho T}{\eta_{0}} \\
& =\frac{2^{d+2} d}{\sqrt{\pi}} \xi^{*} \frac{x_{r}}{\sigma} \frac{\bar{\phi}}{\mathcal{M}} \rho T^{1 / 2} g_{2}
\end{aligned}
$$

have the same formal definition as in molecular gases, that is why we call them the Reynolds $\mathcal{R}$, the Prandtl $\mathcal{P}$ and the Froude number $\mathcal{F}$. Typically the Reynolds number $\mathcal{R}$ describes the ratio of convective to viscous forces. That means if we have small Reynolds number a molecular gas is very viscous e.g. a dust particle in air. High Reynolds numbers indicates that the system is dominated by inertial forces, such a system could be the flow around an airplane. The Prandtl number $\mathcal{P}$ compares the viscosity with the thermal conductivity, that means it is a measurement of the ratio between diffusion of momentum and diffusion of energy. Typically, we find very low Prandtl numbers in liquid metals (e.g. mercury under standard conditions, $\mathcal{P} \approx 0.02$ ) and high Prandtl numbers for very viscous liquids (e.g. glycerol under standard conditions, $\mathcal{P} \approx 11340$ ). In granular gases we find that the Prandtl number is close to the Prandtl number of hard sphere gases $\mathcal{P}_{H S}=\frac{2}{3}$ (see Section 3.4). The dimensionless variables $\mathcal{C}_{\mu}$ and $\mathcal{X}$ do not appear in ordinary gases. The quantity $C_{\mu}$ is related to the "pycnothermal" term $\mu \nabla \rho$ in the heat flux $\boldsymbol{q}$, that couples 
density and thermal flux. This is in principle present also in molecular fluids but the Onsager theorem protects against it [62] yielding $\mathcal{C}_{\mu}=0$. The quantity $\mathcal{X}$ is related to the granular cooling coefficient $\xi$.

At this point the question raises if granular gases behave like ordinary gases in the case of comparable Reynolds and Prandtl number. We discuss the granular Reynolds and Prandtl number below in Section 3.4.

The dimensionless transport coefficients read [56]

$$
\begin{aligned}
\eta & =\eta_{0} \eta^{*} \\
\eta_{0} & =\frac{2+d}{2^{d+1}} \sqrt{\pi} T^{1 / 2} g_{2}^{-1}, \\
\kappa & =\kappa_{0} \kappa^{*} \\
\kappa_{0} & =\frac{d(d+2)^{2}}{2^{2+d}(d-1)} \sqrt{\pi} T^{1 / 2} g_{2}^{-1} .
\end{aligned}
$$

We can rewrite the conservative Eq. (3.17) in the general form

$$
\partial_{t} \boldsymbol{U}+\nabla \cdot \boldsymbol{F}^{c}(\boldsymbol{U})+\nabla \cdot \boldsymbol{F}^{v}(\boldsymbol{U}, \nabla \boldsymbol{U})=\boldsymbol{S},
$$

where we call the super-vector

$$
\boldsymbol{F}^{c}=\left(\begin{array}{c}
\rho \boldsymbol{v} \\
\boldsymbol{v} \rho \boldsymbol{v}^{T}+\mathcal{M}^{-2} p \mathbb{1} \\
\rho H \boldsymbol{v}
\end{array}\right)
$$

the convective flux,

$$
\boldsymbol{F}^{v}=-\frac{1}{\mathcal{R}}\left(\begin{array}{c}
0 \\
\nabla \boldsymbol{v}+(\nabla \boldsymbol{v})^{T}-\frac{2}{d}(\nabla \cdot \boldsymbol{v}) \mathbb{1} \\
\mathcal{M}^{2}\left[\nabla \boldsymbol{v}+(\nabla \boldsymbol{v})^{T}-\frac{2}{d}(\nabla \cdot \boldsymbol{v}) \mathbb{1}\right] \cdot \boldsymbol{v}-\frac{1}{\mathcal{P}} \boldsymbol{q}
\end{array}\right)
$$

the diffusive or viscous flux and

$$
\boldsymbol{S}=\left(\begin{array}{c}
0 \\
\frac{1}{\mathcal{F}} \rho \boldsymbol{a} \\
\frac{1}{\mathcal{F}} \mathcal{M}^{2} \rho \boldsymbol{v} \cdot \boldsymbol{a}-\frac{d}{2} \mathcal{X} \rho T
\end{array}\right)
$$

the source term. If the system is subjected to forces, the forces appear in the source term as well.

We have rewritten the equations in a dimensionless form, so that they look similar to the well known NS equations of molecular gases. Nevertheless, there are some differences from ordinary gases which we will discuss in the next section. 


\subsection{Reynolds, Prandtl and Mach number in granular gases}

In the last section we derived the dimensionless NS equations (3.17) for granular gases in a similar way to the equations of molecular gases. We discuss below the dimensionless variables in Eq. (3.26) in more detail. They appear naturally in the conservative dimensionless from of the NS equations as they do in molecular gases. However there are differences from molecular gases because of the dissipative nature of granular gases. The main difference can be seen from the fact that these numbers are changing with time, in fact they are no constants in granular gases but become variables (apart from the Prandtl number, which stays constant in the $\varepsilon=$ const. model). We first discuss the situation of constant coefficient of restitution. Because the viscosity and the thermal conductivity follow the same power law in $T$, the Prandtl number $\mathcal{P}$, defined in Eq. (3.26), becomes a dimensionless number. Then we can expand the ratio $\frac{\eta^{*}}{\kappa^{*}}$ which appears in the definition of $\mathcal{P}$ in a Taylor series around the elastic particle limit $\varepsilon=1$. This gives the first order of the Prandtl number in granular gases in two and three dimensions

$$
\begin{aligned}
& \mathcal{P}^{2 D}=\frac{1}{4}+\frac{15}{128}(1-\varepsilon)-\frac{9473}{8192}(1-\varepsilon)^{2}+\mathcal{O}\left((1-\varepsilon)^{3}\right) \\
& \mathcal{P}^{3 D}=\frac{4}{15}-\frac{7}{720}(1-\varepsilon)-\frac{160009}{276480}(1-\varepsilon)^{2}+\mathcal{O}\left((1-\varepsilon)^{3}\right) .
\end{aligned}
$$

The textbook definition of Prandtl number [50] for molecular gases is

$$
\mathcal{P}^{*}=\frac{\kappa}{\eta} c_{p},
$$

where $c_{p}$ is the specific heat. If we multiply $\mathcal{P}^{3 D}$ by the heat capacity of a hard-sphere gas $c_{p}^{H S}=\frac{5}{2}, \mathcal{P}$ becomes in the limit of full elasticity $\varepsilon=1$ the well known Prandtl number for hard-spheres gas $\mathcal{P}=\frac{2}{3}$ in three dimension. The Prandtl number in Eq. (3.32) is independent of other parameters and can be well defined even if the system is in a non-steady state.

It is useful to rewrite the Reynolds variable in terms of Mach and Knudsen number

$$
\begin{aligned}
\mathcal{R} & =\frac{m_{p} \rho_{r} v_{r} x_{r}}{\hat{\eta}}, \\
& =\frac{\mathcal{M}}{\mathcal{K}} \frac{g_{2}}{\eta^{*}} E(d),
\end{aligned}
$$

where we used Eq. (3.27) and (2.24), $E(2)=\pi, E(3)=\frac{16}{5}$ are constants.

By Taylor expanding $\frac{1}{\eta^{*}}$ around $\varepsilon=1$ we can write the Reynolds number up to second order

$$
\begin{aligned}
& \mathcal{R}^{2 D}=\frac{\mathcal{M}}{\mathcal{K}} g_{2} \pi\left[1-\frac{15}{64}(1-\varepsilon)-\frac{335}{2048}(1-\varepsilon)^{2}+\mathcal{O}\left((1-\varepsilon)^{3}\right)\right], \\
& \mathcal{R}^{3 D}=\frac{\mathcal{M}}{\mathcal{K}} g_{2} \frac{16}{5}\left[1-\frac{157}{384}(1-\varepsilon)-\frac{1453}{24576}(1-\varepsilon)^{2}+\mathcal{O}\left((1-\varepsilon)^{3}\right)\right] .
\end{aligned}
$$


The Reynolds number in Eq. (3.35) depends on the Mach number. That means that if we can control the Mach number in the system to stay at a certain level, it becomes reasonable to describe the system in terms of the Reynolds number. Typically, the temperature will drastically decrease in a granular gas because of its dissipative nature.

In free cooling system the situation is different. The problem arises that the Mach number is not well defined, which means it is not constant. The temperature drastically decreases and will change the Mach number over time. However we show in Chapter 5 and 6 that the velocity flows are nearly constant. The effective Mach number and thereby the effective Reynolds number increases over time, in fact they vary over many orders of magnitude. This means that a homogeneous granular gas starts in a Stokes flow regime and will turn into an inertial flow regime. Depending on the Knudsen number, a freely cooling system will eventually become turbulent when the effective Mach number is large enough. This means that we cannot find a reasonable reference Mach number $\mathcal{M}$ in freely cooling granular gases ${ }^{2}$. Because the reference of the thermal energy appears only through the definition of the reference Mach number $\mathcal{M}$, we have the freedom to set any number here, the order of magnitude of the thermal velocity will anyway change drastically. Therefore we choose in the case of a freely cooling system a reference $\mathcal{M}=1$ with $v_{t h, r}=v_{t h, 0}$, where $v_{t h, 0}$ is the initial thermal velocity. This means that we measure all convective and thermal velocities in units of the initial thermal velocity.

It is possible to reach steady states in driven granular gases too; here it is reasonable to speak of a Reynolds number in the system. If we drive the system via vibrating walls we introduce energy through shocks and convective fluxes into the system. In this case the thermal velocities are again more or less of the same order of magnitude as the convective velocities. That means that it is reasonable to use a reference Mach number $\mathcal{M}$ equal to unity in this case, too.

\footnotetext{
${ }^{2}$ We describe the importance of the Mach number in the Section 5.1 and in [63].
} 


\section{Computational model}

The numerical treatment of the NS equation plays an important role in numerous fields of research. Especially for engineers the Euler and NS equations are likely to be the most fundamental equations. Within the field of partial differential equations (PDEs) the NS equations are known to be among the most complex equations in physics(excluding general relativity), but they are used in all kinds of hydrodynamic systems like geoscience, astrophysics, magnetohydrodynamic and so on. Indeed, PDEs are at the heart of most continuous descriptions of physical systems, such as fluids, electromagnetic fields or even gravitational fields in astrophysical problems. The necessity to handle such nonlinear coupled partial differential equations leads to wide interest in the numerical treatment of such classes of problems. We are in the position that we can choose among many methods and find the ones which are best suited for our set of NS equations. In this chapter we describe the numerical methods which are suited to the granular NS equations. Briefly, the granular NS equations pose a new kind of challenge with respect to the NS equations for molecular fluids, as the Reynolds number is not constant but changes with time. Thus, a setup where the granular system is roughly incompressible will evolve to a strongly compressible fluids, and further more shock waves will emerge. The physical evolution poses a formidable challenge from the point of view of computational physics. We have devised a method to solve these challenges.

Mathematicians classify PDEs in three different categories. Their behavior can be distinguished in terms of the propagation of information: we find PDEs of hyperbolic, parabolic and elliptic type. The linear advection equation is a prototypical example for hyperbolic equations

$$
\partial_{t} U+v \partial_{x} U=0
$$

where $v=$ const is the velocity of the wave propagation; hyperbolic equations represent causal propagation of information with finite speed. A typical example for a PDE of the 
parabolic type is the heat flux equation

$$
\partial_{t} u+\partial_{x}\left(\kappa \partial_{x} u\right)=0
$$

where $\kappa$ is the diffusion coefficient; parabolic equations correspond to diffusive processes with infinite propagation speed. The irreversible nature of the process is reflected in the first order derivative with respect to time. Finally, the basic example for an elliptic PDE is the Poisson equation

$$
\partial_{x x} u+\partial_{y y} u=\rho(x, y),
$$

where $\rho(x, y)$ is the source term. If the source term is zero, Eq. (4.3) is called the Laplace equation. Elliptic PDEs correspond to equilibrium problems where there is no propagation of information, rather all perturbations have been smoothed out. This is the limit of infinite propagation of information in a PDE of the parabolic type. For all kinds of PDEs a number of different techniques have been developed in the past decades, so that the numerical treatment of each type has become a complex field of research on its own. In order to make the mathematical structure explicit we rewrite the equations (3.17) for the collective vector $\boldsymbol{U}=\boldsymbol{U}(\boldsymbol{r}, t)=(\rho, \rho \boldsymbol{v}, \rho E)$ in the general form of Eq. (3.28)

$$
\partial_{t} \boldsymbol{u}+\nabla \cdot \boldsymbol{F}^{c}(\boldsymbol{u})+\nabla \cdot \boldsymbol{F}^{v}(\boldsymbol{u}, \nabla \boldsymbol{u})=\boldsymbol{S}(\boldsymbol{u}),
$$

where the hyperbolic and parabolic parts of Eq. (4.4) are related to the convective flux $\boldsymbol{F}^{c}$ (Eq. (3.29)) and the viscous or diffusive flux $\boldsymbol{F}^{v}$ (Eq. (3.30)), respectively; these terms have to be handled by different numerical techniques. The intention of this chapter is to give an overview of the complexity of handling such equations. We explain below all methods that are used in our solver.

Briefly what we will discuss is the following. We solve Eq. (3.17), similarly to what is conventionally done for molecular fluids, for the conservative variables $(\rho, \rho \boldsymbol{v}, \rho E)$. Therefore, we employ a finite volume method on a grid of up to $128^{3}$ cells in $3 \mathrm{D}$ (up to $1024^{2}$ in $2 \mathrm{D}$ ) with different boundary conditions. The granular Navier-Stokes equations in conservative form are integrated in each grid cell. Because of Gauss theorem volume integrals become surface integrals in each cell. Each surface integral is performed via Gaussian-Legendre integrals. Our solver contains an operator splitting to calculate the convective flux plus source terms and the diffusive fluxes independently. The interpolation of the variables needed at the Gaussian stencils is performed via a seventh order WENO method [64, 65] and the flux is approximated with the help of a MUSTA scheme [66]. The time integration is carried out explicitly with a second order arbitrary high order derivative (ADER) scheme [67]. For the diffusive flux we implemented the explicit dGRP flux. The entire solver was implemented on a Nvidia's TESLA K20c GPU accelerator with a compute unified device architecture (CUDA) code. 


\subsection{Programming on graphics processing units}

In the last decade it has become more and more prominent to use graphics processing units (GPUs) instead of central processing units (CPUs) in computational physics. The architecture of a GPU differ from a CPUs's, because historically, they were introduced to perform calculation related to linear algebraic operations, which includes the rendering of polygons, and various geometric operations, such as rotation and translation, and later to render 3D computer graphics. This technological process was driven by the video-game industry. Nowadays GPUs are designed to perform a massive number of simple operations using thousand of threads in parallel.

The specialized architecture of GPUs can be used to tackle complex problems in computational physics. The problems that are well suited for parallelization must however satisfy the constraint of the hardware architecture, namely, they must be prone to be reduced to a large number of simple parallel operations. Typically, problems where only local exchanges of information occur are ideally suited. A well known example is the Ising model, which is amenable of efficient GPU implementation [68]. Hydrodynamics is another well suited problem. A technical issue is the small size of the Level 1 (L1)-Cache within GPUs, which makes it necessary to write the code in a way that only few operation are performed in parallel per GPU call. This drives the need for efficient algorithms which perform well when run in parallel. We developed a solver which has been implemented on GPU. We discuss below some points to be aware of when running simulations on GPUs.

Our source code is compiled with the Nvidia CUDA compiler (NVCC) which is based on a $\mathrm{C}++$ compiler. The code was developed in $\mathrm{C}$ and then in a stepwise fashion translated to CUDA. This has the big advantage that debugging is much easier in $\mathrm{C}$ rather than directly on a graphic card, in addition specific CUDA problems are avoided. The straightforward way is then to replace a for loop in C with a kernel in CUDA. The implementation was done on a Kepler K20 card which has 13 parallel calculation units called streaming multiprocessor architecture (SMX), each including 192 single processing units (CUDA cores). It is possible to run the same instruction but with different data on all CUDA cores within one SMX, this kind of parallelization is called single instruction, multiple data (SIMD) and is exactly the kind of parallelization we use within our solver. This means that it is possible to run 13 functions in parallel, or to run one function 13 times in parallel. To achieve a high occupancy of the card one should use 192 threads per block. A function which is executed from a CPU to run on a GPU is called a kernel and one instance of this function is a thread (one thread runs on one CUDA core). The problem arising here is that each SMX has only a total of $64 \mathrm{kB}$ memory for both L1 cache and shared memory. The total amount of L1-Cache is split between all threads running in a SMX, this means that for full occupancy a thread cannot use more than 300 bytes of memory. This makes it necessary to write really small kernels. Typically then the problem appears that many reading and writing steps become necessary. In our case the reading and writing of 
data took however much less processing time than the calculation of the various numerical methods, but this point might be different in simulations with not as many calculation steps such as MD simulations. All fields we use in our simulations are written in a onedimensional field; this has the advantage that the values are in linear sequence inside the memory. Reading from memory cost $100 \mathrm{CPU}$ cycles plus one additional cycle for every additional memory read in a line. If the data are not in one line inside the memory then it will cost again 100 cycles; this is computationally expensive and should be avoided in any case. To save some L1-Cache we suggest the use of precompiler directives. In addition, one should try to reduce the number of calculations; especially divisions are very computationally expensive. The next important issue for achieving a high performance boost on graphics cards is also true for parallelization with CPUs. We obtain a good performance only if the threads can be executed independently from each other. This is the point when local calculations become very important. A coupling between the threads leads typically to a large volume of data exchanged, the situation is even worse if data transfers between different blocks become unavoidable. In hydrodynamic simulation this is possible if no implicit solvers are used. Implicit solvers couple different positions in space (memory) with each other, but the data transfer between them is not efficiently doable. This is the main reason why we avoid the usage of implicit methods. It reveals that it is even possible to implement implicit solver efficiently on GPUs, but we have not implemented such methods so far. The last important issue is the usage of single and double floating point numbers. In our code we implemented precompiler directives to change the numerical precision; simulations using single floating point numbers (32bit) are nearly three times faster than those using double precision (64bit). In our implementation we find that the code is by about 200 times faster on the K20 than on a single core INTEL i7. Parallelization among multiple graphic cards could in principle increase the performance even more. At the moment our simulations are limited by the global memory (RAM) of the graphics card. The largest system size we can simulate contains $144^{3}$ finite volumes in 3D.

\subsection{Overview of the numerical solver}

To handle the NS equations (Eq. (3.17)) we have to build a solver which has access to some state of the art methods. Because our system has no preferential point, nor preferential axis, we always assume Cartesian geometry, and we write the solver on a Cartesian grid with variable step sizes $\Delta x, \Delta y, \Delta z$. The most fundamental question about the numerical solution of PDEs is what general scheme to use to discretize the system. The finite difference method (FDM) directly approximates derivatives by small differences. This is historically the oldest method. The big advantage lies in the simplicity of the method, because it is easy to achieve high order approximation of the derivatives. The main 
issues of the FDM are the stability of the solver and the validation of the conservation laws. Due to the approximations at specific points, the method cannot guarantee that conserved quantities remain constant. This is the main advantage of the finite volume method (FVM). Here we replace the differential equation in Eq.(3.17) by its integral version. The important issue about FVM is that we deal no longer with specific values at certain points but with average values of the quantities inside a finite volume. With the use of Gauss theorem, volume integrals become surface integrals. The complexity of this method lies then in the calculation of the fluxes (that means convective and diffusive) on the surface of each finite volume to update the physical quantities in time. The calculation of fluxes guarantees that there is no deterioration of the conserved quantities because of the method (numerical error due to precision might be neglected here).

The straightforward way to solve a PDE is to split the problem into its components (convective flux, diffusive flux and source term) and calculate each part on its own. This natural way is called operator splitting, there exist however much more complex methods which do not need to split the PDE. We use an operator splitting scheme that solves the coupled convective flux with the source term in the first step, and then calculates the diffusive part in a second step. The convective part is solved with an ADER scheme and the diffusive part is solved with a dGRP scheme. Both schemes are explicit, which is suited for an efficient implementation on a GPU. An explicit scheme calculates the variables $\boldsymbol{u}^{n+1}$ at the new time step from the known values $\boldsymbol{u}^{n}$ of the previous steps and is typically a local scheme: $\boldsymbol{u}^{n+1}=\boldsymbol{u}^{n}+\mathcal{L}\left(\boldsymbol{u}^{n}\right)$. In contrast, an implicit method calculates the variables at the new time step in such a way that the variables fulfill an equation at the new time step implicitly: $\boldsymbol{u}^{n+1}=\boldsymbol{u}^{n}+\mathcal{L}\left(\boldsymbol{u}^{n+\mathbf{1}}\right)$. The implicit method has the disadvantage that it couples the entire calculation domain.

The next important issue is the numerical calculation of the surface integrals, which is performed via a two-point Gaussian-Legendre integration in each dimension. We mentioned before that we store the average values of physical quantities but we also have to calculate the flux on the surface of the finite volumes. The flux is calculated from the values of the quantities at a specific point on the surface. This makes an interpolation step from the average values unavoidable. Here we use the WENO method; a high order upwind, non-oscillating scheme to reconstruct values. After the reconstruction we are faced with a Riemann problem on the surface which has to be handled. We implemented therefore a multi-stage scheme, which is an iterative scheme to approximate the exact Riemann solution. Figure 4.1 shows a flow chart of the numerical solver to give an overview of the main computational steps. In this chapter we discuss all parts of our solver in detail. 


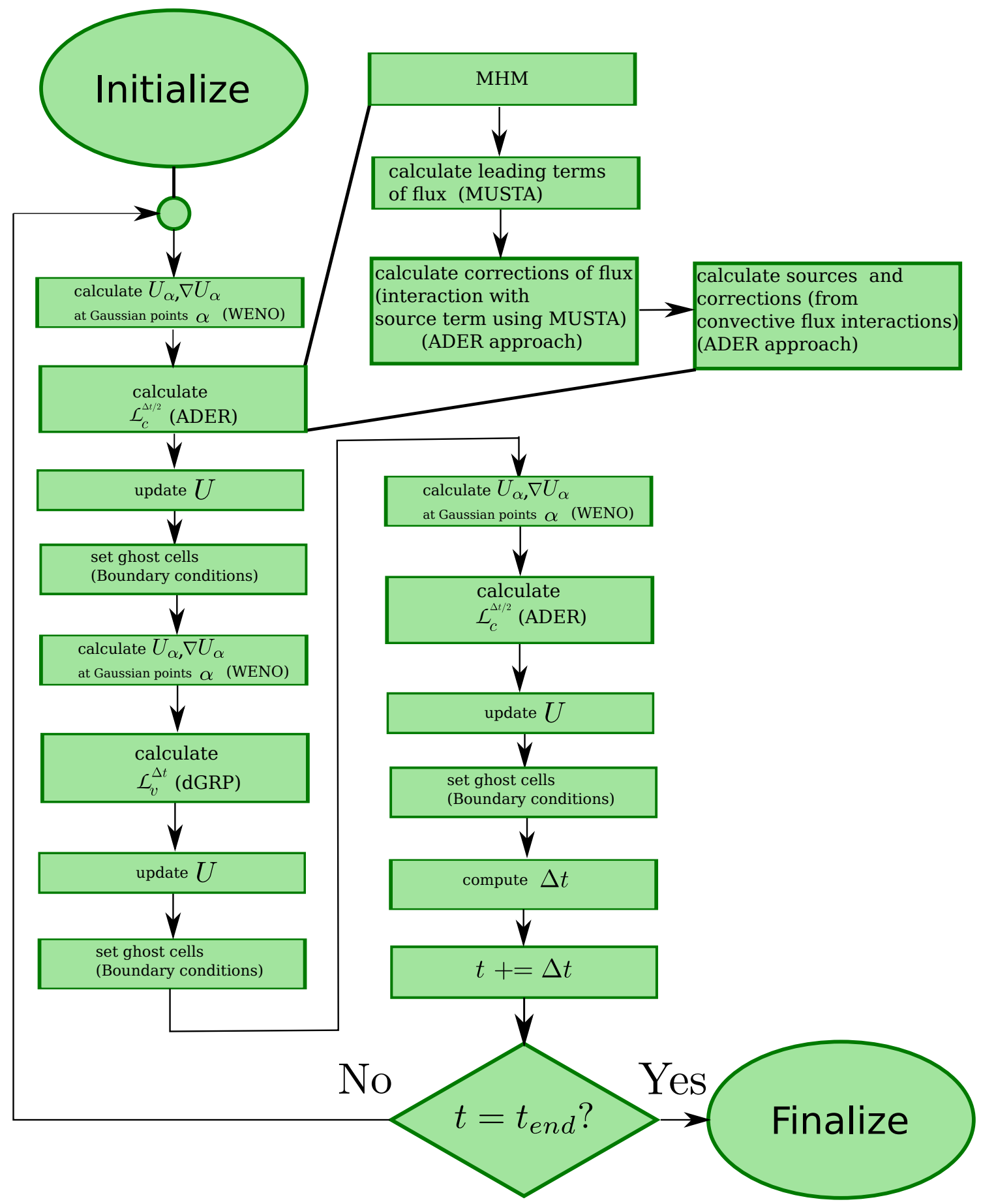

Figure 4.1: Flow chart of the structure of the algorithm for the direct numerical simulations. 


\subsection{Operator splitting}

A common approach to solve coupled, inhomogeneous convective-diffusive problems like the case at hand is to use operator splitting schemes [69-71]. The book by Holden [71] gives a good introduction to operator splitting methods and goes beyond the methods we implemented for our solver. Let's assume the following initial value problem (IVP)

$$
\begin{aligned}
& \partial_{t} \boldsymbol{U}+\nabla \cdot \boldsymbol{F}^{c}(\boldsymbol{U})+\nabla \cdot \boldsymbol{F}^{v}(\boldsymbol{U}, \nabla \boldsymbol{U})=\boldsymbol{S}(\boldsymbol{U}) \\
& \boldsymbol{U}(\boldsymbol{r}, t=0)=\boldsymbol{U}_{0}(\boldsymbol{r}),
\end{aligned}
$$

where $\boldsymbol{U}(\boldsymbol{r}, t)$ is the vector of conservative variables. $\mathcal{L}_{c}$ denotes the exact solution operator associated with the inhomogeneous hyperbolic problem

$$
\partial_{t} \boldsymbol{U}+\nabla \cdot \boldsymbol{F}^{c}(\boldsymbol{U})=\boldsymbol{S}(\boldsymbol{U})
$$

and $\mathcal{L}_{v}$ be the exact solution operator associated with the parabolic problem

$$
\partial_{t} \boldsymbol{U}+\nabla \cdot \boldsymbol{F}^{v}(\boldsymbol{U}, \nabla \boldsymbol{U})=0 .
$$

For a small time step $\Delta t$ we can obtain the solution of Eq. (4.5) in two steps. First we apply $\mathcal{L}_{c}^{\Delta t}$ to gain the intermediate solution

$$
\boldsymbol{U}^{*}(\boldsymbol{r})=\mathcal{L}_{c}^{\Delta t} \boldsymbol{U}(\boldsymbol{r}, t)
$$

and then we apply $\mathcal{L}_{v}^{\Delta t}$ on $\boldsymbol{U}^{*}$ to gain the full solution after $\Delta t$

$$
\boldsymbol{U}(\boldsymbol{r}, t+\Delta t)=\mathcal{L}_{v}^{\Delta t} \boldsymbol{U}^{*}(\boldsymbol{r})=\mathcal{L}_{v}^{\Delta t} \mathcal{L}_{c}^{\Delta t} \boldsymbol{U}(\boldsymbol{r}, t)
$$

The operator splitting method in (4.9) and (4.10) is of order $\mathcal{O}(\Delta t)$ accurate in each time step if the solution is sufficiently smooth [71]. Formally, a second order method is achieved if instead we use the operator [71]

$$
\boldsymbol{U}(\boldsymbol{r}, t+\Delta t)=\left(\mathcal{L}_{c}^{\Delta t / 2} \mathcal{L}_{v}^{\Delta t} \mathcal{L}_{c}^{\Delta t / 2}\right) u(\boldsymbol{r}, t)
$$

The solution method used in Eq. (4.11) is called multiplicative operator splitting (MOS) and is implemented in the numerical solver we use. An alternative MOS splitting is the $\mathcal{L}_{c}$ surrounded by two $\mathcal{L}_{v}$. For the sake of completeness we want to mention another famous alternative splitting method which is called additive operator splitting (AOS)

$$
\boldsymbol{U}(\boldsymbol{r}, t+\Delta t)=\left(\frac{1}{2} \mathcal{L}_{c}^{\Delta t} \mathcal{L}_{v}^{\Delta t}+\frac{1}{2} \mathcal{L}_{v}^{\Delta t} \mathcal{L}_{c}^{\Delta t}\right) \boldsymbol{U}(\boldsymbol{r}, t)
$$

which is also of second order. In our work we choose to implement the MOS for the reasons of computational convenience that we describe in the following. It would seem that an 
implementation of the AOS in Eq. (4.12) would be more suited for GPU parallelization than the MOS in Eq. (4.11) because of its additive character which can be parallelized among different calculation units. But in fact the opposite is true. The AOS requires four calculations, while the MOS requires only three. We need twice as much memory in AOS to save the intermediate solutions than the memory needed in MOS by just updating the current state. In addition, each operator $\mathcal{L}_{c}, \mathcal{L}_{v}$ is calculated by a parallelization among a grid of millions of finite volumes, that means that there are no resources (CUDA cores) available for a further parallelization, so that it is not possible to gain advantage of the additive character of the AOS. In general MOS are more accurate than AOS [71] but it is worth mentioning that the operators in MOS do not in general commute, which puts the AOS on a more solid ground, as there are no ambiguities in the choice of the time ordering of the operators.

\subsection{Finite-volume method}

We solve the IVP (3.28) via a FVM and follow the standard procedure for FVM. It is also possible to directly approximate the differentials appearing in the IVP (3.28) on a grid, which would lead to the FDM. The big advantage of the FVM is that it takes into explicit account the existence of conservation theorems and the conservation of the corresponding quantities. That means that physical quantities like density can only be transported from one volume to another (apart from the source terms) which leads to a method which conserves these quantities. The FDM instead does not conserve these quantities and leads to artificial numerical sinks/sources. That is why our method of choice is the FVM. The basic idea of FVM is to divide the computational domain in finite size volumes and solve the problem locally in each sub-cell. The smooth function $\boldsymbol{U}$ is replaced by the average of $\boldsymbol{U}$ in each finite volume and the evolution of these average values is calculated. A very good reference that introduces finite-volume numerical methods for hydrodynamic models is the book by Toro [72].

We consider a system of $N_{x}, N_{y}, N_{z}$ finite volumes in $x$-, $y$ - and $z$-direction respectively. A finite volume (cuboid computational cell) $V_{i j k}$ in $3 \mathrm{D}$

$$
\begin{aligned}
& V_{i j k}=\left[x_{i-1 / 2}, x_{i+1 / 2}\right] \times\left[y_{j-1 / 2}, y_{j+1 / 2}\right] \times\left[z_{k-1 / 2}, z_{k+1 / 2}\right], \\
& i \in\left(0, N_{x}\right), \quad j \in\left(0, N_{y}\right), \quad k \in\left(0, N_{z}\right)
\end{aligned}
$$

has linear sizes $\Delta x, \Delta y, \Delta z$ and volume $V_{i j k}=\Delta x \Delta y \Delta z$. The integration of Eq. (3.28) over the finite volume $V_{i j k}$ is performed by means of Gauss theorem

$$
\begin{aligned}
\int_{V_{i j k}} \nabla \cdot \boldsymbol{F} \mathrm{dV} & =\int_{\partial V_{i j k}} \boldsymbol{F} \mathrm{d} \boldsymbol{S} \\
\partial_{t} \overline{\boldsymbol{U}}_{i j k} & =-\frac{1}{\left|V_{i j k}\right|} \int_{\partial V_{i j k}} \boldsymbol{F}^{c} \mathrm{~d} \boldsymbol{S}+\frac{1}{\left|V_{i j k}\right|} \int_{\partial V_{i j k}} \boldsymbol{F}^{v} \mathrm{~d} \boldsymbol{S}+\frac{1}{\left|V_{i j k}\right|} \int_{V_{i j k}} \boldsymbol{S} \mathrm{dV},
\end{aligned}
$$


where

$$
\overline{\boldsymbol{U}}_{i j k} \equiv \frac{1}{\left|V_{i j k}\right|} \int_{V_{i j k}} \boldsymbol{U} \mathrm{dV}
$$

is the spatial average of $\boldsymbol{U}$ in the finite volume $V_{i j k}$ at time $t$, and $\mathrm{d} \boldsymbol{S}=\boldsymbol{n} \mathrm{dS}$. The value of the field at the updated time is achieved via the time integral of Eq. (4.15) over the interval $\Delta t$

$$
\begin{aligned}
\overline{\boldsymbol{U}}_{i j k}(t+\Delta t)= & \boldsymbol{U}_{i j k}(t)-\frac{1}{\left|V_{i j k}\right|} \int_{t}^{t+\Delta t} \int_{\partial V_{i j k}} \boldsymbol{F}^{c} \mathrm{~d} \boldsymbol{S}+ \\
& +\frac{1}{\left|V_{i j k}\right|} \int_{t}^{t+\Delta t} \int_{\partial V_{i j k}} \boldsymbol{F}^{v} \mathrm{~d} \boldsymbol{S}+\frac{1}{\left|V_{i j k}\right|} \int_{t}^{t+\Delta t} \int_{V_{i j k}} \boldsymbol{S} \mathrm{dV} .
\end{aligned}
$$

The solution in Eq. (4.18) is in principle still exact. The finite-volume scheme may be interpreted as resulting from the integration of the equations in space and time on the control volume $V_{i j k} \times[t, t+\Delta t]$. For the cuboid domain $V_{i j k}$ problem, Eq. (4.18) can be explicitly written as

$$
\begin{aligned}
\overline{\boldsymbol{U}}_{i j k}(t+\Delta t)= & \overline{\boldsymbol{U}}_{i j k}(t)-\frac{\Delta t}{\Delta x}\left(\boldsymbol{F}_{i+1 / 2}^{c}-\boldsymbol{F}_{i-1 / 2}^{c}\right) \\
& -\frac{\Delta t}{\Delta y}\left(\boldsymbol{F}_{j+1 / 2}^{c}-\boldsymbol{F}_{j-1 / 2}^{c}\right)-\frac{\Delta t}{\Delta z}\left(\boldsymbol{F}_{k+1 / 2}^{c}-\boldsymbol{F}_{k-1 / 2}^{c}\right) \\
& +\frac{\Delta t}{\Delta x}\left(\boldsymbol{F}_{i+1 / 2}^{v}-\boldsymbol{F}_{i-1 / 2}^{v}\right)+\frac{\Delta t}{\Delta y}\left(\boldsymbol{F}_{j+1 / 2}^{v}-\boldsymbol{F}_{j-1 / 2}^{v}\right) \\
& +\frac{\Delta t}{\Delta z}\left(\boldsymbol{F}_{k+1 / 2}^{v}-\boldsymbol{F}_{k-1 / 2}^{v}\right)+\Delta t \boldsymbol{S}_{i j k},
\end{aligned}
$$

with the space averages of physical fluxes over cell faces

$$
\begin{aligned}
\boldsymbol{F}_{i+1 / 2}^{\boldsymbol{c}} & =\frac{1}{\Delta t} \frac{1}{\Delta y \Delta z} \int_{t}^{t+\Delta t} \int_{y_{j-1 / 2}}^{y_{j+1 / 2}} \int_{z_{k-1 / 2}}^{z_{k+1 / 2}} \boldsymbol{F}^{c}\left(\boldsymbol{U}\left(x_{i+1 / 2}, y, z, \tau\right)\right) \mathrm{d} y \mathrm{~d} z \mathrm{~d} \tau, \\
\boldsymbol{F}_{j+1 / 2}^{c} & =\frac{1}{\Delta t} \frac{1}{\Delta x \Delta z} \int_{t}^{t+\Delta t} \int_{x_{i-1 / 2}}^{x_{i+1 / 2}} \int_{z_{k-1 / 2}}^{z_{k+1 / 2}} \boldsymbol{F}^{c}\left(\boldsymbol{U}\left(x, y_{j+1 / 2}, z, \tau\right)\right) \mathrm{d} x \mathrm{~d} z \mathrm{~d} \tau, \\
\boldsymbol{F}_{k+1 / 2}^{c} & =\frac{1}{\Delta t} \frac{1}{\Delta x \Delta y} \int_{t}^{t+\Delta t} \int_{x_{i-1 / 2}}^{x_{i+1 / 2}} \int_{y_{j-1 / 2}}^{y_{j+1 / 2}} \boldsymbol{F}^{c}\left(\boldsymbol{U}\left(x, y, z_{k+1 / 2}, \tau\right)\right) \mathrm{d} x \mathrm{~d} y \mathrm{~d} \tau, \\
\boldsymbol{F}_{i+1 / 2}^{\boldsymbol{v}} & =\frac{1}{\Delta t} \frac{1}{\Delta y \Delta z} \int_{t}^{t+\Delta t} \int_{y_{j-1 / 2}}^{y_{j+1 / 2}} \int_{z_{k-1 / 2}}^{z_{k+1 / 2}} \boldsymbol{F}^{\boldsymbol{v}}\left(\boldsymbol{U}\left(x_{i+1 / 2}, y, z, \tau\right), \nabla \boldsymbol{U}\left(x_{i+1 / 2}, y, z, \tau\right)\right) \mathrm{d} y \mathrm{~d} z \mathrm{~d} \tau, \\
\boldsymbol{F}_{j+1 / 2}^{\boldsymbol{v}} & =\frac{1}{\Delta t} \frac{1}{\Delta x \Delta z} \int_{t}^{t+\Delta t} \int_{x_{i-1 / 2}}^{x_{i+1 / 2}} \int_{z_{k-1 / 2}}^{z_{k+1 / 2}} \boldsymbol{F}^{\boldsymbol{v}}\left(\boldsymbol{U}\left(x, y_{j+1 / 2}, z, \tau\right), \nabla \boldsymbol{U}\left(x, y_{j+1 / 2}, z, \tau\right)\right) \mathrm{d} x \mathrm{~d} z \mathrm{~d} \tau, \\
\boldsymbol{F}_{k+1 / 2}^{\boldsymbol{v}} & =\frac{1}{\Delta t} \frac{1}{\Delta x \Delta y} \int_{t}^{t+\Delta t} \int_{x_{i-1 / 2}}^{x_{i+1 / 2}} \int_{y_{j-1 / 2}}^{y_{j+1 / 2}} \boldsymbol{F}^{\boldsymbol{v}}\left(\boldsymbol{U}\left(x, y, z_{k+1 / 2}, \tau\right), \nabla \boldsymbol{U}\left(x, y, z_{k+1 / 2}, \tau\right)\right) \mathrm{d} x \mathrm{~d} y \mathrm{~d} \tau, \\
\boldsymbol{S}_{i j k} & =\frac{1}{\Delta x \Delta y \Delta z} \int_{t}^{t+\Delta t} \int_{x_{i-1 / 2}}^{x_{i+1 / 2}} \int_{y_{j-1 / 2}}^{y_{j+1 / 2}} \int_{z_{k-1 / 2}}^{z_{k+1 / 2}} \boldsymbol{S}(\boldsymbol{U}(x, y, z, \tau)) \mathrm{d} x \mathrm{~d} y \mathrm{~d} z \mathrm{~d} \tau .
\end{aligned}
$$


The challenge posed by the finite-volume method is to find high order approximations for the surface integrals of the fluxes and the sources in space and time. Once this is done the state $\overline{\boldsymbol{U}}_{i j k}$ can be updated in time.

\subsection{Gauss-Legendre integration}

Typically surface integrals as they appear in Eq. (4.19) are performed via Gauss-Legendre integration. This is a numerical approximation scheme that evaluates the integral of a function $f(x)$ by means of a weighted sum of values of the function at specific points $f\left(x_{i}\right)$ called "stencils" [73]. We can write the approximation as

$$
\int_{-1}^{1} f(x) \mathrm{d} x \approx \sum_{i=1}^{n} \omega_{i} f\left(x_{i}\right)
$$

where the integration range $[-1,1]$ is a convention and typically requires a transformation of the variable. For all linear space integrations we use two Gaussian integration points

$$
\frac{1}{\Delta \xi} \int_{\xi_{i-1 / 2}}^{\xi_{i+1 / 2}} \varphi(\xi) \mathrm{d} \xi \approx 0.5\left[\varphi\left(\xi_{i}-\Delta \xi /(2 \sqrt{3})\right)+\varphi\left(\xi_{i}+\Delta \xi /(2 \sqrt{3})\right)\right]
$$

It becomes therefore necessary to approximate, or reconstruct, the value of $\boldsymbol{U}(\boldsymbol{r})$ at the Gaussian integration point $\left(x_{\alpha}, y_{\beta}, z_{\gamma}\right)$ from discrete data points at $\left(x_{i}, y_{j}, z_{k}\right)$. In principle, there are two ways to achieve the needed reconstruction: (i) a genuine multidimensional reconstruction [74, 75], and (ii) a dimension by dimension reconstruction [74, 76, 77]. The multidimensional reconstruction uses multidimensional polynomials $p^{i}(x, y, z)$ as the basis to predict the value $\boldsymbol{U}_{\alpha, \beta, \gamma}$. The dimension by dimension reconstruction instead uses one-dimensional polynomials $p^{i}(x)$. Dimension by dimension reconstruction can only be done along one-dimensional manifolds and takes typically three (two) reconstruction sweeps in three (two) dimensions. The multidimensional reconstruction methods needs only one reconstruction sweep to obtain the value $\boldsymbol{U}_{\alpha, \beta, \gamma}$ in all dimensions. Figure 4.2 shows a schematic sketch of the reconstruction methods in $2 \mathrm{D}$ to get an impression of the complexity of the problem. Figure 4.3 displays the Gaussian stencil points in 3D which have to be calculated. We strongly suggest the use of a dimension by dimension reconstruction which is much simpler to implement and less computational expensive than the multidimensional reconstruction. We briefly explain below how we achieve our Gaussian integration points $g_{\alpha}$ via a dimension-by-dimension reconstruction (see Section 4.6 for details on the reconstruction method). In a first step we perform a polynomial reconstruction in the center of the surface of a finite volume. Let's assume for simplicity that the first step is done in the $x$-direction and leads to the approximate value $U_{i+1 / 2}$. In the next step, it would be straightforward to reconstruct in the $y$-direction followed by a reconstruction in the $z$-direction. In principle, the reconstruction directions $x, y, z$ 


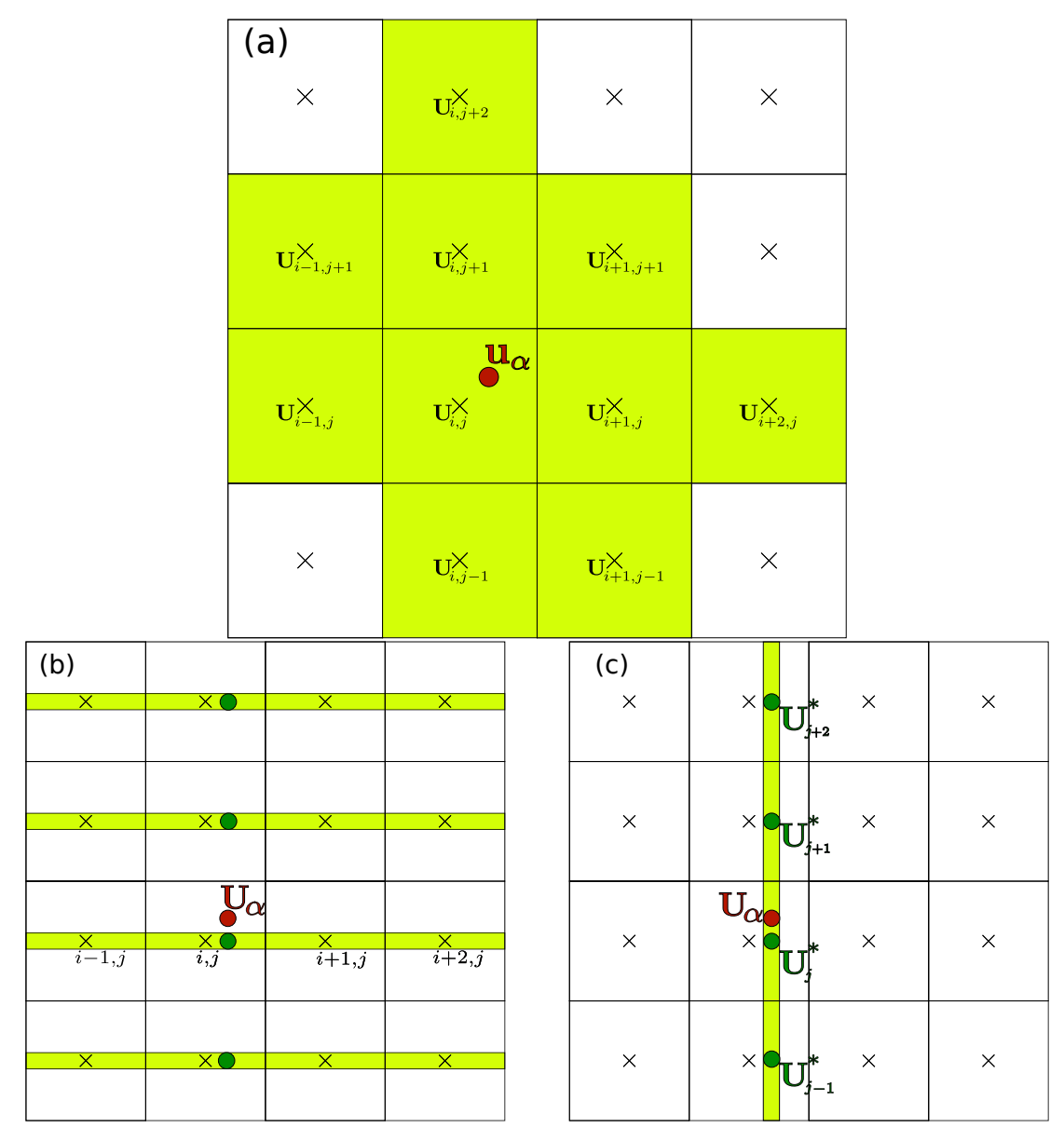

Figure 4.2: A schematic sketch of the multidimensional reconstruction method (a) and the dimension by dimension method (b) and (c) in two dimension. The reconstruction is performed from central points $\boldsymbol{U}$. The multidimensional method uses stencil points in all available dimensions to construct a polynomial from where the Gaussian stencil point can be calculated. In contrast the dimension by dimension reconstruction is performed in two sweeps. In the first sweep the black stencils are used to construct a one-dimensional polynomial from where the green intermediate points are calculated. During the second sweep we achieve from the green intermediate stencils the final Gaussian stencil point $\boldsymbol{U}_{\alpha, \beta, \gamma}$ along a second one-dimensional reconstruction. 


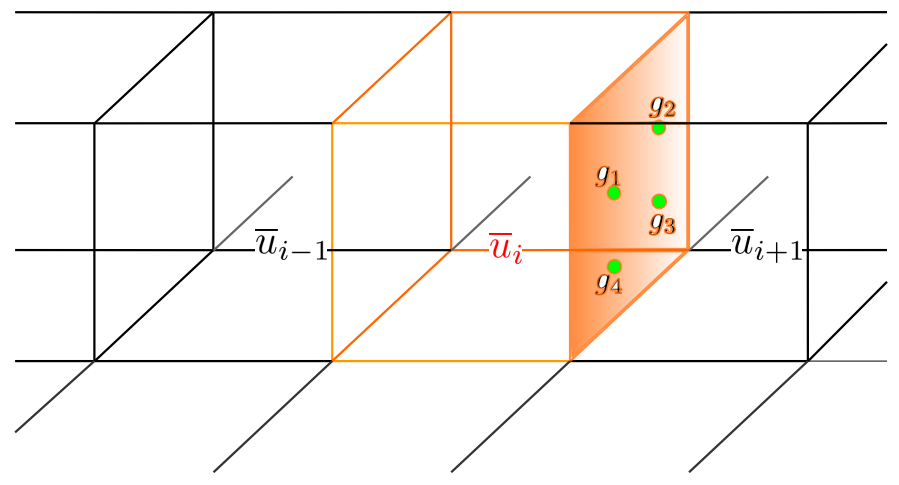

Figure 4.3: Sketch of the Gaussian stencil points on the surface of a 3D finite volume.
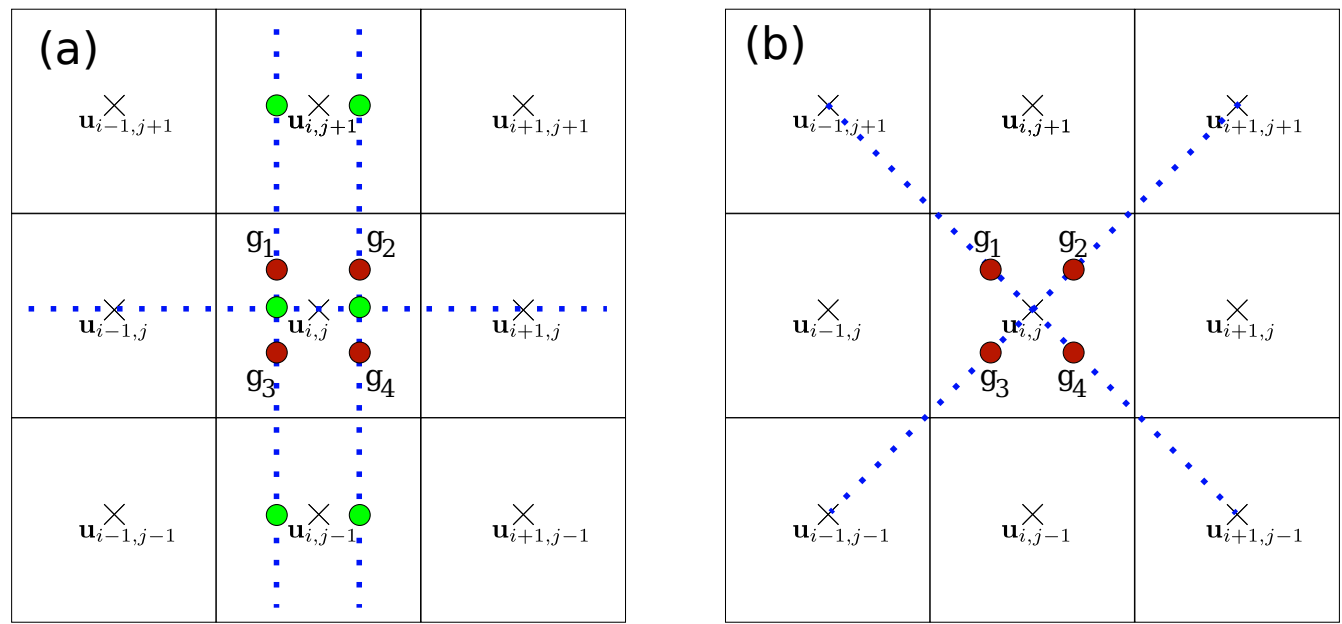

Figure 4.4: Reconstruction on a 2D surface to obtain the stencils $g_{i}$. Panel (a) displays the reconstruction in 2 steps using an intermediate point (green circle). Panel (b) displays the reconstruction in one step. 
can be performed in any order, but typically, the first step is the reconstruction on the surface. This method would need three reconstruction steps via dimension-by-dimension reconstruction to gain the Gaussian integration point. Typically the Gaussian points lie on the connecting line between diagonal finite volumes. In this case one can use this direction for a reconstruction line; by doing so one is able to reduce the number of calculations. Figure 4.4 sketches the two methods. The disadvantage of the two-step reconstruction is a slight loss of accuracy. We suggest to use the two-point reconstruction whenever possible for two reasons. First, the reconstruction step is heavily expensive in terms of computational costs, so performance is the first argument against the threepoint reconstruction. The second argument is a more physically one. Once the first reconstruction leads to an intermediate point on the surface, there are two possibilities to achieve the reconstructed value at the Gaussian integration point via the three-step reconstruction. None of these roads is preferred against the other, but the result will end up in a slightly different value. The two-step reconstruction avoids this artificial symmetry breaking.

As mentioned before, we are using two Gaussian points to approximate the integral. Using more than two Gaussian points drastically increases the computational cost and is not needed in our case. On graphics cards the flux calculation on a Gaussian integration point takes the most performance during a time step (about $70 \%$ of the load). That means that using two Gaussian integration points is a compromise between performance and accuracy and leads to a $\mathcal{O}\left((\Delta x)^{4}\right)$ approximation. In 3D, four Gaussian integration points are required to approximate one surface integral of a finite volume. Increasing the approximation to three Gaussian integration points would lead to a total of 9 Gaussian integration points on a surface in 3D. Following [66] the two-point Gaussian quadrature is the best compromise in terms of accuracy and computational cost for the number of reconstruction stencils we use.

\subsection{Polynomial reconstruction: WENO method}

In the last decades a lot of research has been done on polynomial reconstruction. A naive reconstruction method can be described as taking the data from different cells to create a polynomial and then calculate the value of this polynomial at the missing space point. One would expect that more cells increase the order of the polynomial and lead to more correct or stable solutions. Sadly this naive Ansatz fails. The reason for this failure is that polynomials tend to oscillate ${ }^{1}$, especially if a discontinuity appears as it is the case at locations of high gradients and shocks. A spline reconstruction leads typically to better results but still has similar problems. We present here the WENO reconstruction, which has become the state of the art reconstruction method since it was developed in

\footnotetext{
${ }^{1} \mathrm{~A}$ polynomial of order $n$ has up to $(n-1)$ extrema which makes oscillating behavior even more likely.
} 
1994 [64]. The basic idea is to use different possible reconstruction polynomials and use
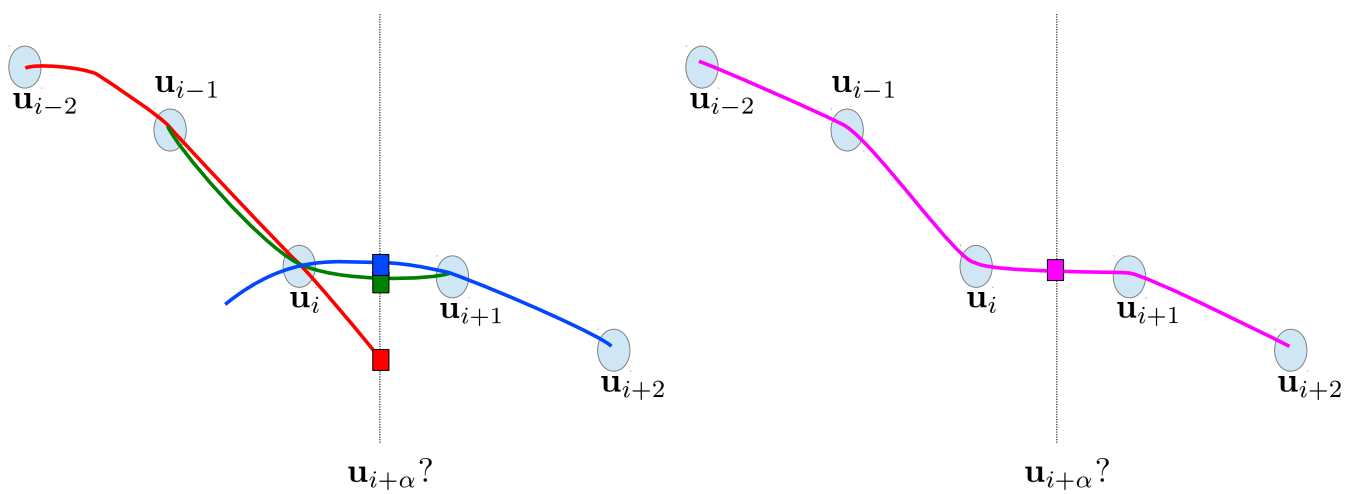

Figure 4.5: The problem of reconstruction of the value $\boldsymbol{U}_{i+\alpha}$ at $x=x_{\alpha}$ as an example of $r=3$. The left panel shows the small construction polynomials $p^{1}, p^{2}, p^{3}$ and the right panel shows the large construction polynomial $P$. The figure shows the problem for a FDM for the sake of simplicity; in the FVM the exact points have to replaced with the averages over the corresponding cell.

all of them with different weights for a reconstruction step. The weights here depend on the "smoothness" of a polynomial, that means the polynomials with less oscillating behavior and without steep gradients are preferred in the reconstruction step. Originally, the WENO method was developed by Liu et al. [64] and shortly after it was further developed by Jiang and Shu [65]. The polynomial reconstruction can be performed using the exact values $\boldsymbol{U}_{i}$, like they appear natively in FDM, or for average values $\overline{\boldsymbol{U}}_{i}$ as they appear in FVM. In a FVM the possible reconstruction polynomials have to fulfill the average condition in Eq. (4.16). We describe the method for the $x$-direction, which can be easily extended to other directions ${ }^{2}$. Imagine a given point $\overline{\boldsymbol{U}}_{i}$ and the missing point $\boldsymbol{U}_{i+\alpha}, \alpha \in(-\Delta x / 2, \Delta x / 2)$.

Consider $r$ polynomials $p^{j}(x)$ of order $r-1$ which contain $\overline{\boldsymbol{U}}_{i}$. We call the polynomials $p^{j}(x)$ the 'small reconstruction polynomials' because they only take a small number of stencils in the reconstruction step. We name stencil the known values which are used to create a polynomial; they might be exact values at a certain point (FDM) or averages over a region $(\mathrm{FVM})$. The first polynomial $p^{1}(x)$ is built from the $(r-1)$ stencils $\overline{\boldsymbol{U}}_{i-r+1}, \overline{\boldsymbol{U}}_{i-r+2}, \cdots, \overline{\boldsymbol{U}}_{i}$, the second polynomial $p^{2}(x)$ is build from the stencils $\overline{\boldsymbol{U}}_{i-r+2}, \overline{\boldsymbol{U}}_{i-r+3}, \cdots, \overline{\boldsymbol{U}}_{i+1}$ and so on. That means that we need for the construction of all $r$ small polynomials a total of $(2 r-1)$ stencils, namely $\overline{\boldsymbol{U}}_{i-r+1}, \overline{\boldsymbol{U}}_{i-r+2}, \cdots, \overline{\boldsymbol{U}}_{i+r-2}, \overline{\boldsymbol{U}}_{i+r-1}$. In addition, it is possible to use all $(2 r-1)$ stencils to create a polynomial $P(x)$. Because all $(2 r-1)$ stencils are used in the reconstruction step, we call $P(x)$ the 'large polynomial'. Figure 4.5 shows the small polynomials in the left panel and the large polynomial in the right panel. In a finite difference description, the polynomials are created from the conditions $p^{j}(x)=u_{k}, k \in\{i-r+j, \cdots, i-1+j\}$, in the finite volume description, the polynomials have to fulfill the condition $\frac{1}{\Delta x} \int_{k-1 / 2}^{k+1 / 2} p^{j}(x) \mathrm{d} x=\overline{\boldsymbol{U}}_{k}, k \in\{i-r+j, \cdots, i-1+j\}$.

\footnotetext{
${ }^{2}$ We described in Section 4.5 the advantage of dimension by dimension reconstruction.
} 
Every polynomial $p^{j}$ is able to approximate the value at $x_{\alpha}$. If the polynomials are smooth, this approximation is of $r$ th order accuracy [65], otherwise the accuracy is drastically reduced. To find the final approximation we use a linear combination of the different $p^{j}$

$$
\boldsymbol{U}_{i+\alpha}=\sum_{k=0}^{r-1} \omega_{k} p^{k}\left(\boldsymbol{U}_{i+\alpha}\right) .
$$

To achieve the essentially non-oscillatory behavior we have to choose weights with respect to the smoothness of the $p^{j}$. This means we want to avoid oscillations and discontinuities, this is feasible if not smooth polynomials have a weight close to zero. However, in smooth regions we want to keep sure that the approximation (4.22) matches with the result of the large polynomial $P(x)$ at $x=x_{\alpha}$

$$
P\left(x_{i+\alpha}\right)=\sum_{k=0}^{r-1} d_{k} p^{k}\left(\boldsymbol{U}_{i+\alpha}\right) .
$$

Simple algebra leads to an expression for the weights $d_{k}$ in Eq. (4.23). Because the $d_{k}$ lead to high accuracy only if the domain is smooth we call them optimal weights. The question arises how to choose the $\omega_{k}$ in Eq. (4.22), so that they are close to $d_{k}$ in smooth regions while the non-oscillatory property is achieved. The lengthy details of how to achieve the high order accuracy in the approximation $\boldsymbol{U}_{\alpha}$ can be found in $[64,65]$. The nonlinear weights $\omega_{k}$ in Eq. (4.22) are found [64] to be

$$
\omega_{k}=\frac{\alpha_{k}}{\sum_{j} \alpha_{j}}
$$

where

$$
\alpha_{k}=\frac{d_{k}}{\left(\epsilon+\beta_{k}\right)^{2}}, \quad k=0,1,2, \cdots, r-1,
$$

and where $\beta_{k}$ are the so-called smoothing parameters. $\epsilon$ is a small number to prevent the denominator from vanishing in a numerical simulation and must be chosen in such a way that $\epsilon^{2}$ is still above the numerical precision. A typical value is $\epsilon=10^{-6}$ which is what we choose in our simulation, too. Incidentally, we notice that the exponent of two in the denominator of Eq. (4.25) can be also chosen larger; in [64] they suggest to take an exponent equal to $r$. However, we will use an exponent of two in our simulation as it is taken quite often in the literature $[65,66,75]$, otherwise one has to keep in mind to correct the stabilization parameter $\epsilon$. We are left with the question how to define the smoothing parameters $\beta_{k}$, which in turn means how to measure the smoothness of a polynomial $p^{k}$. We follow [65] and calculate the $\beta_{k}$ as the sum of $L_{2}$-norms of all derivatives of $p^{j}$ in the surrounding area of the center point $u_{i}$, which is quite a natural way to measure the 
smoothness

$$
\beta_{k} \equiv \sum_{l=1}^{r-1}(\Delta x)^{2 l-1} \int_{x_{i-\Delta x / 2}}^{x_{i+\Delta x / 2}}\left(\frac{\partial^{l}}{\partial x^{l}} p_{k}\right)^{2} \mathrm{~d} x .
$$

The prefactor $(\Delta x)^{2 l-1}$ removes the $\Delta x$ dependency of $\beta_{k}$. We use for all reconstruction steps a 7 -th order WENO method, which means $r=4$; the coefficients needed to implement this method are listed in [66]. It is worth mentioning that typically the WENO method is one of the most performance intense methods within a numerical solver; on a CPU it is responsible for up to $90 \%$ of the overall calculation time. That means that it is really useful to use an efficient implementation of the method.

On GPUs we find that the WENO method does not take so much of the overall calculation time if it is implemented in an efficient way. The biggest challenge on GPUs is the small L1-cache. Some points to be aware of are: (i) try to precalculate as much as possible with the help of preprocessor directives; (ii) do as few calculation as possible; (iii) do not reach the numerical precision of (double) floating point numbers. We suggest to normalize the stencil points $\overline{\boldsymbol{U}}_{j}, j \in(r-2+1, \ldots, r+2-1)$ with the value of the central point $\overline{\boldsymbol{U}}_{i}$ because it fulfills (i)-(iii). In fact the normalization step is unavoidable in our case because we have to deal with extremely different orders of magnitude in our quantities.

The reader can find the implementation, in the $\mathrm{C}$ programming language, of the reconstruction from average values $\overline{\boldsymbol{U}}_{i}$ at a Gaussian integration point in Appendix 10.2. In addition, a MATHEMATICA ${ }^{\circledR}$ source code that generates the WENO method at any rebuilding point $x_{\alpha}$ to seventh oder accuracy for reconstruction from averages and from exact values is also given in Appendix 10.3.

\subsection{WENO method for derivatives}

In the last section, the WENO method for the reconstruction of $\boldsymbol{U}_{i+\alpha}$ was introduced. The reconstruction of the derivative $\boldsymbol{U}^{\prime}$ at position $x_{i+\alpha}$ can be done in a similar way, which means using the same building polynomials. The order of accuracy of $\boldsymbol{U}_{i+\alpha}^{\prime}$ is one order below the accuracy of $\boldsymbol{U}_{i+\alpha}$, because taking the derivative reduces the polynomial order by one. To calculate the derivatives, the condition (4.23) must now be modified in such a way that the derivatives of small polynomials match the derivative of the large polynomial

$$
P^{\prime}\left(x_{i+\alpha}\right)=\sum_{k=0}^{r-1} d_{k} p^{\prime k}\left(\boldsymbol{U}_{i+\alpha}\right) .
$$

This leads to the optimal weights for the superposition of the $p^{\prime k}$. We calculate the nonlinear weights $\omega_{k}$ again from Eq. (4.24) and (4.25). Smoothing factors $\beta_{k}$ for the derivative reconstruction are obtained from the sum of the $L_{2}$-norms of all derivatives of 
$p^{\prime j}$

$$
\beta_{k}=\sum_{l=2}^{r-1}(\Delta x)^{2 l-1} \int_{x_{i-\Delta x / 2}}^{x_{i+\Delta x / 2}}\left(\frac{\partial^{l}}{\partial x^{l}} p_{k}\right)^{2} \mathrm{~d} x .
$$

The MAthematica ${ }^{\circledR}$ code, given in Appendix 10.3 to calculate the WENO reconstruction at a position $x_{i+\alpha}$, includes the option to calculate the derivatives at the same position.

\subsection{Riemann problem}

With the WENO method we introduced a method to reconstruct the physical quantities on the surface of a finite volume. The WENO method rebuilds values $\overline{\boldsymbol{U}}_{i \pm \alpha}$ with $\alpha \in$ $[-1 / 2,+1 / 2]$. Therefore we can rebuild the surface point $\boldsymbol{U}_{i+1 / 2}$ either using $\overline{\boldsymbol{U}}_{i}$ or $\overline{\boldsymbol{U}}_{i+1}$ as the center point for the reconstruction polynomials. Numerically we achieve two different values at the same position. There is no preference in the direction of the reconstruction and therefore it is ambiguous which of the two reconstructed values has to be chosen. This raises the question, what is the best approximation of the variables at the surface? We

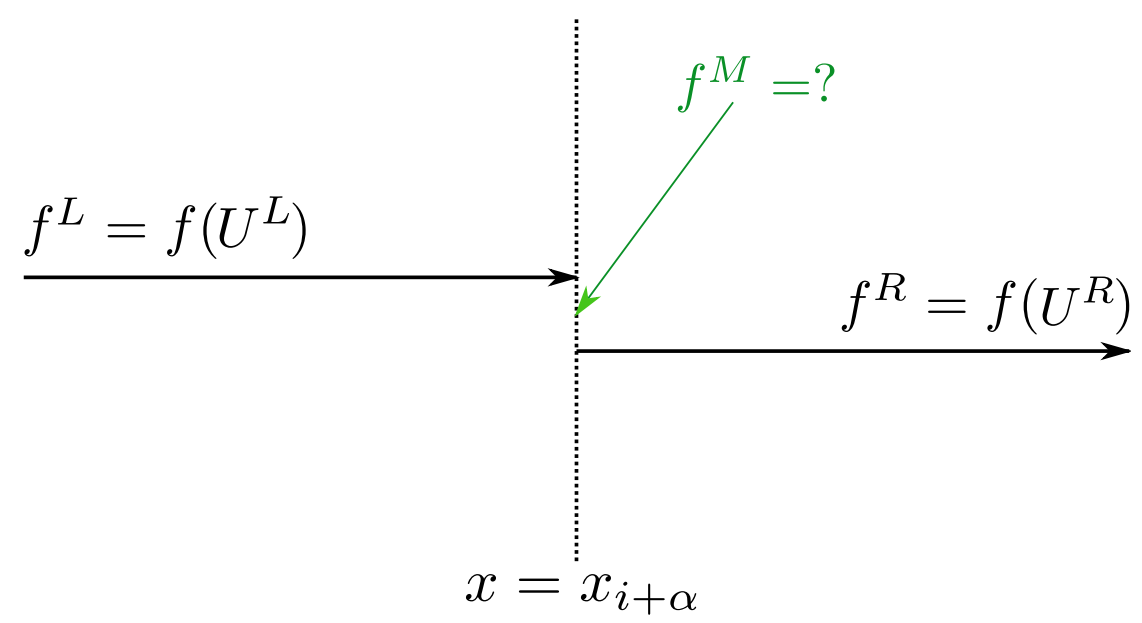

Figure 4.6: Riemann problem: $U^{L}$ and the resulting flux $f^{L}$ is the initial solution $(t=0)$ to the left of $x_{i+\alpha}, U^{R}$ and the corresponding flux $f^{R}$ is initial solution to the right of $x_{i+\alpha}$. The problem is to determine the initial flux $f^{M}$ at position $x+x_{i+\alpha}$.

name $\boldsymbol{U}^{L}$ the value reconstructed from the center point $\overline{\boldsymbol{U}}_{i}$ and we name $\boldsymbol{U}^{R}$ the value reconstructed from the center point $\overline{\boldsymbol{U}}_{i+1}$.

We consider the scalar problem in which $U^{L}$ is the valid reconstruction at $i+1 / 2-\epsilon$ and $U^{R}$ is the valid solution at $i+1 / 2+\epsilon$, where $\epsilon \rightarrow 0$. This leads to an IVP with a discontinuity at the surface. The problem is sketched in Figure 4.6. As a first step let's consider the following linear homogeneous IVP

$$
\partial_{t} U(x, t)+a \partial_{x} U(x, t)=0
$$




$$
U(x, t=0)=\left\{\begin{array}{lll}
U^{L}, & \text { if } & x<0 \\
U^{R}, & \text { if } & x>0 .
\end{array}\right.
$$

The PDE and initial conditions in Eq. (4.29) are called the Riemann problem. A very good reference about the Riemann problem and related numerical aspects is [72]. The exact solution to the Riemann problem for the Euler equations is a lengthy calculation for which we refer the reader to [72]. In the following we give a simple, introductory illustration of the resolution methods for the Riemann problem.

We introduce the definition of characteristics or characteristic curves. A characteristic $x(t)$ is a curve in phase space along which a PDE becomes an ordinary differential equation (ODE), typically this is the case if a variable $U(x(t), t)$ becomes time independent along this curve

$$
\frac{\mathrm{d} U}{\mathrm{~d} t}=\frac{\partial U}{\partial t}+\frac{\mathrm{d} x}{\mathrm{~d} t} \frac{\partial U}{\partial x}=0 .
$$

Then the curve satisfies the ODE

$$
\frac{\mathrm{d} x}{\mathrm{~d} t}=a .
$$

$a$ is called the characteristic speed. If we start with an initial point, the value evolves along such a characteristic curve, see Fig. 4.7. In the one dimensional Riemann problem for the

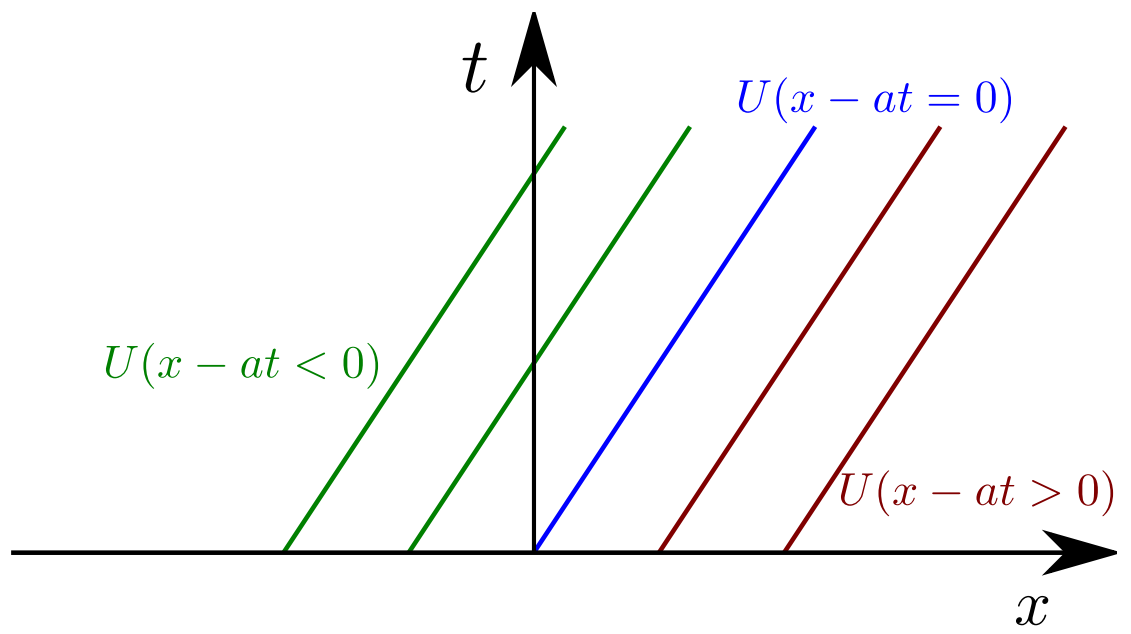

Figure 4.7: Characteristics of the linear advection equation $\partial_{t} U+a \partial_{x} U=0$ with $a>0$ and a discontinuity at $x=0$, different characteristic correspond to different constants. On each characteristic the value $U$ is constant.

linear advection equation in Eq. (4.29) the discontinuity $x=0$ follows also a characteristic in time, therefore the solution is simply

$$
U(x, t)=u_{0}(x-a t)=\left\{\begin{array}{lll}
U^{L}, & \text { if } & x-a t<0 \\
U^{R}, & \text { if } & x-a t>0 .
\end{array}\right.
$$


The corresponding characteristic of this problem are sketched in Fig. 4.7. This means that we will observe a wave emanating from the origin, which carries a jump discontinuity in $u$ and propagating with speed $a$. If we extend the linear advection equation in Eq. (4.29) to $m$ dimensions, we obtain a set of $m$ hyperbolic PDEs of the form

$$
\partial_{t} \boldsymbol{U}+\underline{\mathbf{A}} \cdot \partial_{x} \boldsymbol{U}=0
$$

The coefficient matrix $\underline{\mathbf{A}}$ contains $m$ real eigenvalues $\lambda_{i}$, which are related to a characteristic curve satisfying the condition $\frac{\mathrm{d} x}{\mathrm{~d} t}=\lambda_{i}$ for every $i=1, \ldots, m$. For a discontinuity in Eq. (4.33), which is indeed the Riemann problem in Eq. (4.29), we find that now $m$ waves are emanating from the origin, all carrying a discontinuity with a characteristic speed $\lambda_{i}$. Between each pair of characteristics we find a well defined state $\boldsymbol{U}_{i}$. Figure 4.8 shows the

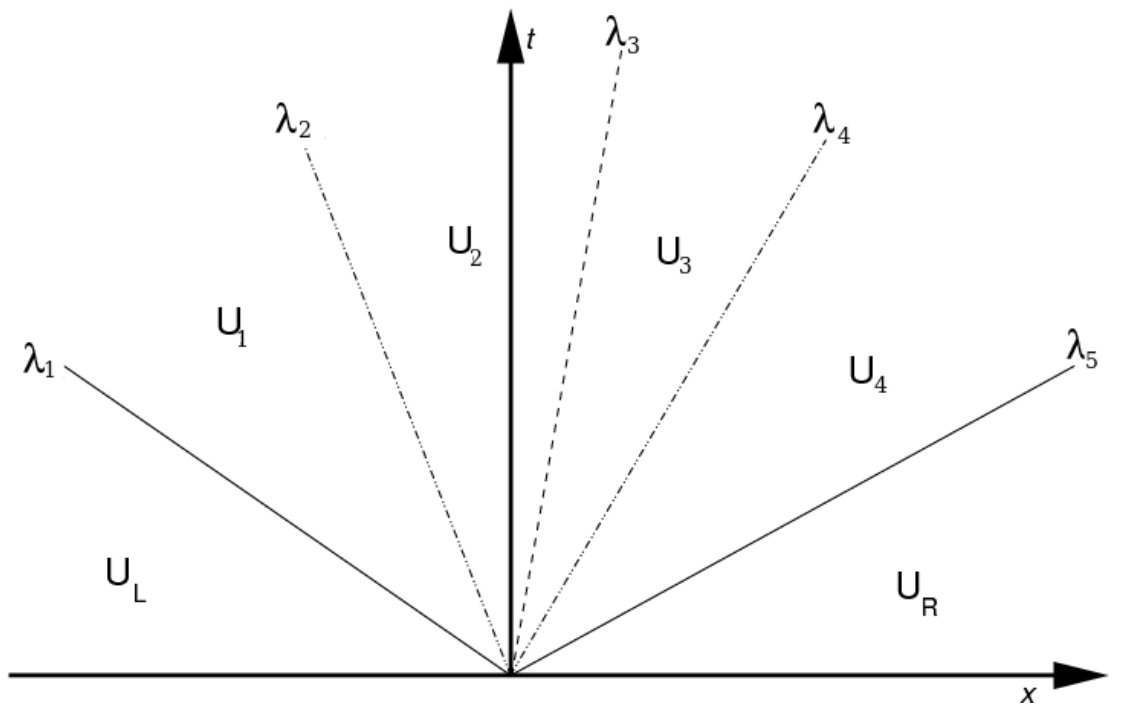

Figure 4.8: Picture of a Riemann fan. The characteristics shown distinguish the different states from each other.

space-time evolution of the different states, which are separated by characteristics. This figure is called the Riemann fan. If the governing equations becomes nonlinear

$$
\partial_{t} \boldsymbol{U}(x, t)+\partial_{x} \boldsymbol{A}(\boldsymbol{U})=0,
$$

we find that the characteristics which we see in the Riemann fan in Fig. 4.8, will no longer be straight lines but become arbitrary curves. The typical way to solve such equation is to solve the easier linearized problem

$$
\partial_{t} \boldsymbol{U}(x, t)+\underline{\mathbf{J}} \cdot \partial_{x} \boldsymbol{U}=0
$$

where we call $\left.\underline{\mathbf{J}} \equiv \nabla_{\boldsymbol{U}} \boldsymbol{A}(\boldsymbol{U})\right|_{\boldsymbol{U}=\mathbf{0}}$ the Jacobian of the problem. The linearized problem is of course only valid for short times. The state of the art method to include higher order terms is the class of schemes collectively known as the ADER schemes [67, 78-82], which 
is discussed in Section 4.12.

Because our goal is to understand the time evolution at $x=0$, we have to figure out which lines the characteristics follow in the $x-t$ space. The main task of a numerical solver is to calculate the intermediate states between the characteristics. This process is called "opening" the Riemann fan, which means that the solver is able to take different intermediate states into account. A simple upwind solver [72] will choose only between $\boldsymbol{U}^{L}$ and $\boldsymbol{U}^{R}$ as an approximation of the solution at $x=0$; such a solver is not able to "open" the Riemann fan. Another class of simple solver are centered solvers [72], which take typically some mean of the left and the right value $\boldsymbol{U}^{L}, \boldsymbol{U}^{R}$ to approximate the solution at $x=0$, however, they do not "open" the Riemann fan correct. More advanced Riemann solvers are able to open the Riemann fan in a better way, which means they take the different intermediate states into account. The big advantage of the MUSTA approach, which we use in our solver, is that we can numerically open the Riemann-fan without the knowledge of the underlying hyperbolic problem. The MUSTA is a general approach, which combines the simplicity of centered schemes and the good accuracy of the Godunov methods. For further details on the MUSTA approach see Section 4.11.

The nonlinear inhomogeneous IVP

$$
\begin{aligned}
& \partial_{t} \boldsymbol{U}(x, t)+\partial_{x} \boldsymbol{F}(\boldsymbol{U}(x, t))=\boldsymbol{S}(\boldsymbol{U}) \\
& \boldsymbol{U}(x, t=0)=\left\{\begin{array}{lll}
\boldsymbol{U}^{L}, & \text { if } & x<0 \\
\boldsymbol{U}^{R}, & \text { if } & x>0 .
\end{array}\right.
\end{aligned}
$$

is related to Eq. (4.29) and is the problem we have to face for the coupled system of convective fluxes and source terms (see Section 4.3). Godunov derived the solution of Eq. 4.36 for the Euler equations [83] without sources; the calculation can be found in Russian in [72]. Keep in mind that Eq. (4.36) is nonlinear, which means that the exact solver might be very complicated or even not available, therefore it is advisable to use approximate nonlinear Riemann solvers. In addition an approximative Riemann solver drastically improves the performance although it was shown that approximated Riemann solvers are limited to second order accuracy in time [72].

The solution $\boldsymbol{U}^{\text {God }}=\boldsymbol{U}(0,0)$ of (4.36) is typically called the Godunov state. The perhaps most common Riemann solvers are the Riemann solver of Roe [84] and the Riemann solver of Osher $[85,86]$. There are a variety of advanced and modified versions of these two Riemann solvers available nowadays; an overview of these modifications can be found in [72]. Actually, we are not interested in the Godunov state itself but in the flux $\boldsymbol{F}^{M}=\boldsymbol{F}(\boldsymbol{U}(0,0))$. Once the Godunov-state is known the flux follows $\boldsymbol{F}^{M}\left(\boldsymbol{U}^{\mathrm{God}}\right)$. But there are also methods available to calculate the flux at $(x=0, t=0)$ without the intermediate step of calculating the Godunov state. A famous solver to obtain directly an approximation of the intercell flux is the approximate Riemann solver invented by Harten, Lax and van Leer (HLL) [87]. In fact the HLL solver builds the basis for an entire family of HLL-Riemann-solver namely HLLE [88], HLLM [89] and the famous HLLC [90]. These 
fluxes are based on the Godunov method and lead to good accuracy of the intercell flux. The disadvantage is the complexity of solvers of the HLL-family especially because knowledge of the eigenstructure of the problem is needed within the solver, which may lead to complex algebraic problems. As mentioned before we are using the simpler alternative of the MUSTA approach. We will describe in the following Sections how we combine the MUSTA approach and the ADER scheme to solve the problem given in Eq. (4.36).

\subsection{MUSCL-Hancock method}

The MHM is routinely used today. MUSCL stands for Monotone Upstream-Centered Scheme. The scheme is based on the explicit conservative formula

$$
\boldsymbol{U}_{i}^{n+1}=\boldsymbol{U}_{i}^{n}-\frac{\Delta t}{\Delta x}\left[\boldsymbol{F}_{i+1 / 2}-\boldsymbol{F}_{i-1 / 2}\right]
$$

for the general conservation law

$$
\partial_{t} \boldsymbol{U}+\partial_{x} \boldsymbol{F}(\boldsymbol{U})=0
$$

The important point is that this method includes a reconstruction step at the surface and a local time evolution step to construct a fully discrete, second-order accurate scheme implementing Eq. (4.37) [72, chapter 13.4.2]. The scheme consists of three steps:

(i) reconstruction of the variables on the boundary, in our case via a WENO method, to gain $\boldsymbol{u}^{L}$ and $\boldsymbol{u}^{R}$;

(ii) local time evolution of $\boldsymbol{U}^{L}, \boldsymbol{U} R$ by half a time step $\Delta t / 2$ according to

$$
\begin{aligned}
\overline{\boldsymbol{U}}^{L} & =\boldsymbol{U}^{L}+\frac{1}{2} \frac{\Delta t}{\Delta x}\left(\boldsymbol{F}\left(\boldsymbol{U}^{L}\right)-\boldsymbol{F}\left(\boldsymbol{U}^{R}\right)\right), \\
\overline{\boldsymbol{U}}^{R} & =\boldsymbol{U}^{R}+\frac{1}{2} \frac{\Delta t}{\Delta x}\left(\boldsymbol{F}\left(\boldsymbol{U}^{L}\right)-\boldsymbol{F}\left(\boldsymbol{U}^{R}\right)\right) .
\end{aligned}
$$

(iii) solve the piece-wise constant Riemann problem

$$
\begin{aligned}
& \partial_{t} \boldsymbol{U}+\partial_{x} F(\boldsymbol{U})=0 \\
& \boldsymbol{U}=\left\{\begin{array}{lll}
\overline{\boldsymbol{U}}^{L}, & \text { if } & x<0 \\
\overline{\boldsymbol{U}}^{R}, & \text { if } & x>0 .
\end{array}\right.
\end{aligned}
$$

We implement this method in our code to solve the problem (4.38). 


\subsection{Centered fluxes}

Centered fluxes are obtained directly from the initial data and do not need the solution of the Riemann problem

$$
F_{i+1 / 2}=F\left(u_{i}, u_{i+1}\right)
$$

Thereby it is possible to see them as low order approximations to the solution of the Riemann problem. The two most common centered fluxes are the Lax-Friedrich flux

$$
F_{i+1 / 2}^{L F}=\frac{1}{2}\left[F\left(u_{i}\right)+F\left(u_{i+1}\right)\right]-\frac{1}{2} \frac{\Delta x}{\Delta t}\left(u_{i+1}-u_{i}\right)
$$

and the Lax-Wendroff flux

$$
\begin{aligned}
F_{i+1 / 2}^{L W} & =F\left(u_{i+1 / 2}^{1 / 2}\right) \\
u_{i+1 / 2}^{1 / 2} & =\frac{1}{2}\left(u_{i}+u_{i+1}\right)-\frac{1}{2} \frac{\Delta t}{\Delta x}\left[F\left(u_{i+1}\right)-F\left(u_{i}\right]\right.
\end{aligned}
$$

The first-order centered scheme (FORCE) flux is a centered flux, which was derived in [91]. It turns out that the FORCE flux is the arithmetic mean of the Lax-Friedrich and LaxWendroff flux.

$$
F_{i+1 / 2}^{F O R C E}=\frac{1}{2}\left(F_{i+1 / 2}^{L F}+F_{i+1 / 2}^{L W}\right)
$$

The FORCE flux is a monotone flux with an optimal handling of numerical dissipation [92] and numerical dispersion [93] and therefore it is a very good choice of a flux which does not make explicit use of the propagation of information with waves.

Numerical dissipation is the general effect produced by a solver that cannot maintain in time a solution with steep gradients in the absence of physical dissipation. That means that an initial jump in the solution will smooth out over time and will lead to inaccurate results. Numerical dispersion seeds oscillations in the system and affects the stability of the simulation. These numerical problems are caused by numerical imprecisions in the approximations of derivatives of the variable. Numerical dissipation is the direct result of even-order derivatives, while numerical dispersion is the direct result of oddorder derivatives. Typically, the leading term of the truncation error will lead mainly to dissipative behavior in the numerical solution if it is an even-order derivative term and to dispersion behavior if it is an odd-order term. A good benchmark to numerical dispersion is the linear advection equation in Eq. (4.29) and a good benchmark to numerical dissipation is the heat flux equation

$$
\partial_{t} U+a \partial_{x x} U=0
$$



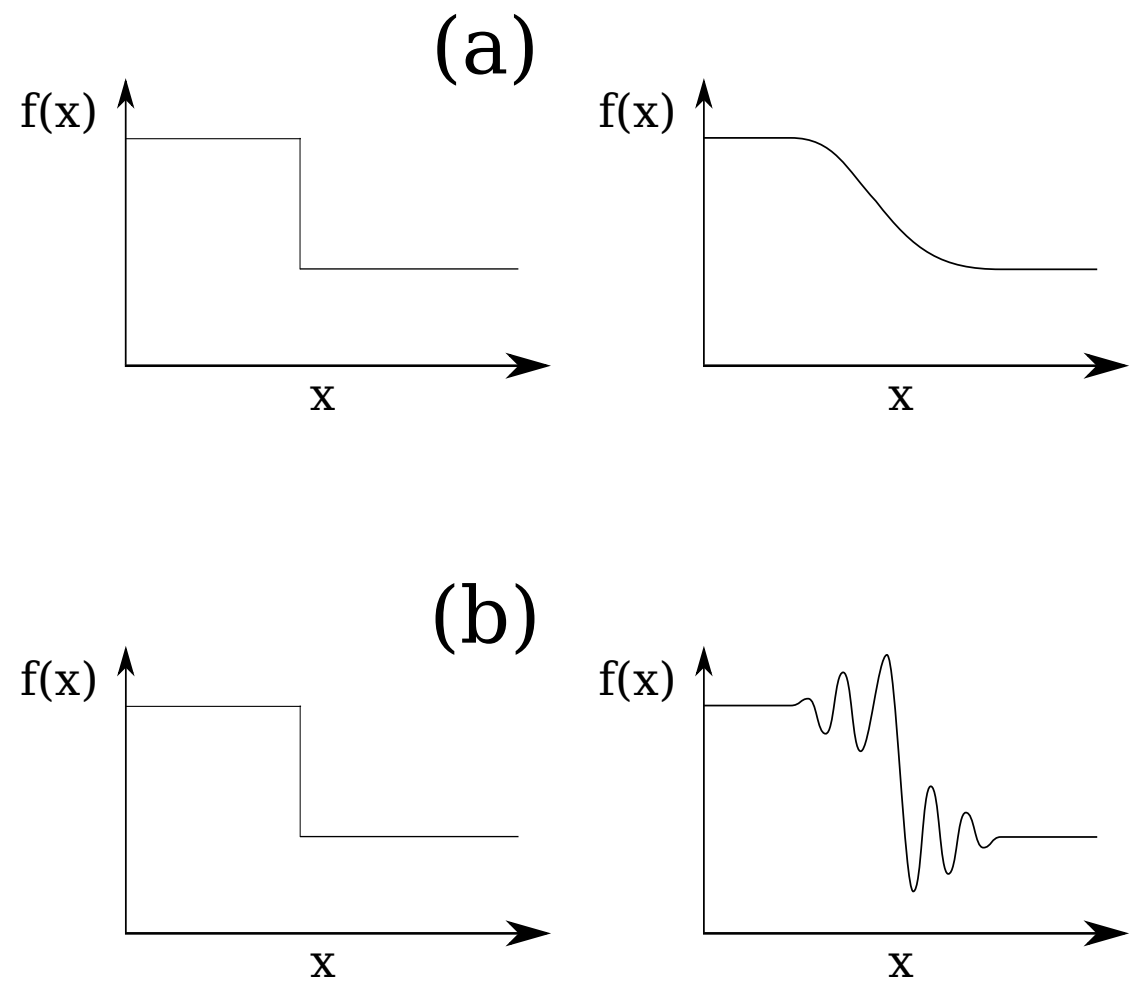

Figure 4.9: Numerical errors which appear during approximation. Panel (a) displays the effect of numerical dissipation and panel (b) displays the oscillations caused by numerical dispersion

Figure 4.9 displays the solution because of this numerical errors. We use the FORCE flux as a predictor and corrector flux within our MUSTA scheme, which is described in detail in the next section.

\subsection{MUSTA flux}

In this section we introduce the MUSTA [66] scheme to solve the Riemann problem

$$
\begin{aligned}
& \partial_{t} \boldsymbol{U}(x, t)+\partial_{x} \boldsymbol{F}(\boldsymbol{U}(x, t))=0 \\
& \boldsymbol{U}(x, t)=\left\{\begin{array}{lll}
\boldsymbol{U}_{L}, & \text { if } & x<0 \\
\boldsymbol{U}_{R}, & \text { if } & x>0 .
\end{array}\right.
\end{aligned}
$$

The key idea of the scheme is to open the Riemann fan by numerical approximation (see also Section 4.8) instead of finding an analytical solution as it is done in the HLL-family solvers. In the MUSTA approach the big advantage is that the eigenstructure of the flux does not have to be calculated explicitly.

This technique considers in a first step the local evolution of the values $\boldsymbol{U}_{L}$ and $\boldsymbol{U}_{R}$ in the area of the boundary at $x=0$, and a numerically correction of the initial data in a second step. Eventually this converges against an asymptotic value which lies in the 
correct intermediate state of the Riemann fan. Let $k$ be the number of local time evolution stages in the MUSTA scheme. At each stage $l \leq k$ we iteratively perform:

(i) flux evaluation

$$
\boldsymbol{F}_{i+1 / 2}^{(l)}=\boldsymbol{F}^{F O R C E}\left(\boldsymbol{U}_{L}^{(l)}, \boldsymbol{U}_{R}^{(l)}\right)
$$

(ii) opening of the Riemann fan

$$
\begin{aligned}
& \boldsymbol{U}_{L}^{(l+1)}=\boldsymbol{U}_{L}^{(l)}-\frac{\Delta t}{\Delta x}\left[\boldsymbol{F}_{i+1 / 2}^{(l)}-\boldsymbol{F}\left(\boldsymbol{U}_{L}^{(l)}\right)\right] \\
& \boldsymbol{U}_{R}^{(l+1)}=\boldsymbol{u}_{R}^{(l)}-\frac{\Delta t}{\Delta x}\left[\boldsymbol{F}\left(\boldsymbol{U}_{R}^{(l)}\right)-\boldsymbol{F}_{i+1 / 2}^{(l)}\right]
\end{aligned}
$$

(iii) goto step (i) if $l \neq k$.

The time step $\Delta t$ in the MUSTA scheme is not necessarily the same as the global time step. We find empirically

$$
\Delta t=C_{f l} \frac{1}{k w_{\max }},
$$

where $C_{f l}$ is the global Courant number, $k$ the number of stages in the scheme and $w_{\max }$ is the fastest wave propagation. Furthermore, it turns out, that even three local time evolution stages are enough to achieve a stable scheme in our case. In the literature modification and generalization are published [94, 95]. We have tested these modifications within our granular NS solver but we do not find more accurate nor more stable results than the MUSTA scheme presented here.

\subsection{ADER scheme}

The ADER scheme is a method to obtain high order approximations of $\boldsymbol{U}_{i+1 / 2}(\tau)$ and $\boldsymbol{F}_{i+1 / 2}(\tau)$ without the need of a numerical quadrature in time. In the last decade considerable work on ADER methods has been done, for further information see [67, 78-82]. We assume the IVP in Eq. (4.36), which is the IVP we have to handle inside our solver

$$
\begin{aligned}
& \partial_{t} \boldsymbol{U}(x, t)+\partial_{x} \boldsymbol{F}(\boldsymbol{U}(x, t))=\boldsymbol{S}(\boldsymbol{U}) \\
& \boldsymbol{U}(x, t=0)=\left\{\begin{array}{lll}
\boldsymbol{U}^{L}, & \text { if } & x<0 \\
\boldsymbol{U}^{R}, & \text { if } & x>0 .
\end{array}\right.
\end{aligned}
$$

Using a FVM (see Section 4.4) we can write formally the solution of (4.51) as

$$
\boldsymbol{U}_{i}(t+\Delta t)=\boldsymbol{U}_{i}(t)-\frac{\Delta t}{\Delta x}\left[\boldsymbol{F}_{i+1 / 2}-\boldsymbol{F}_{i-1 / 2}\right]+\Delta t \boldsymbol{S}_{i},
$$


where $\boldsymbol{U}_{i}$ is the spatial average at time $\tau$

$$
\boldsymbol{U}_{i}=\frac{1}{\Delta x} \int_{x_{i-1 / 2}}^{x_{i+1 / 2}} \boldsymbol{U}(x, \tau) \mathrm{d} x
$$

$\boldsymbol{F}_{i+1 / 2}$ is the time-integral average at the interface $x=x_{i+1 / 2}$

$$
\boldsymbol{F}_{i+1 / 2}=\frac{1}{\Delta t} \int_{t}^{t+\Delta t} \boldsymbol{F}(\boldsymbol{U}(x, t, \tau)) \mathrm{d} \tau
$$

and $\boldsymbol{S}_{i}$ is the volume-integral average in $V$

$$
\boldsymbol{S}_{i}=\frac{1}{\Delta t} \frac{1}{\Delta x} \int_{x_{i-1 / 2}}^{x_{i+1 / 2}} \int_{t}^{t+\Delta t} \boldsymbol{S}(\boldsymbol{U}(x, t, \tau)) \mathrm{d} x \mathrm{~d} \tau
$$

The basic idea of the ADER approach is replacing $\boldsymbol{U}_{i+1 / 2}=\boldsymbol{U}_{i+1 / 2}(\tau)$ with its Taylor series in time

$$
\boldsymbol{U}_{i+1 / 2}(\tau)=\boldsymbol{U}_{i+1 / 2}(0)+\tau \partial_{t} \boldsymbol{U}_{i+1 / 2}(0)+\mathcal{O}\left(\tau^{2}\right) .
$$

We implemented a second order ADER scheme in our solver, but in principle any arbitrarily high order approximation can be obtained with this method. From now on we will show the second order scheme, but the generalization is straightforward. The leading term is the solution of the classical Riemann problem, which is the Godunov state (see also Section 4.8)

$$
\begin{aligned}
& \partial_{t} \boldsymbol{U}(x, t)+\partial_{x} \boldsymbol{F}(\boldsymbol{U}(x, t))=0 \\
& \boldsymbol{U}(x, t=0)=\left\{\begin{array}{lll}
\boldsymbol{U}^{L}, & \text { if } & x<0 \\
\boldsymbol{U}^{R}, & \text { if } & x>0 .
\end{array}\right.
\end{aligned}
$$

The source term does not contribute at time $\tau=0$ and thereby does not affect the Godunov state. The higher order time derivatives are replaced with spatial derivatives via the Cauchy-Kowalewski procedure [80]. In principle the procedure is as follows: starting from a known time derivatives we take an additional derivative of the PDE in Eq. (4.51) with respect to time. Then we switch the chronology of mixed space-time derivative and replace the time derivative with the already known solution. With the help of a bit algebra this leads to a set of equations where we can express higher order time derivatives with higher order spatial derivatives. With the help of the WENO method we are able then to calculate the needed spatial derivaties.

Because we are not interested in the Godunov state itself but in the flux at the boundary 
$x_{i+1 / 2}$, we use the ADER scheme to obtain a Taylor series in time of the flux [67]

$$
\boldsymbol{F}_{i+1 / 2}(\tau)=\boldsymbol{F}_{i+1 / 2}(0)+\tau \partial_{t} \boldsymbol{F}_{i+1 / 2}(0)+\mathcal{O}\left(\tau^{2}\right)
$$

To achieve a second order space-time method we use the mid-point integration rule, which means we set $\tau=\frac{\Delta t}{2}$ in the time integrals. The leading term $\boldsymbol{F}_{i+1 / 2}(0)$ is calculated via the MUSTA-flux for the problem in Eq. (4.57) (see Section 4.11). To obtain the correction terms in the flux we follow the idea of the Cauchy-Kowalewski procedure to express time derivative via space derivatives. The time derivative of the flux can be expressed as

$$
\begin{aligned}
\partial_{t} \boldsymbol{F} & =\underline{\mathbf{J}} \partial_{t} \boldsymbol{U}, \\
\underline{\mathbf{J}} & =\nabla_{\boldsymbol{U}} \boldsymbol{F}, \\
\partial_{t} \boldsymbol{U} & =-\partial_{x} \boldsymbol{F}+\boldsymbol{S},
\end{aligned}
$$

where $\underline{\mathbf{J}}$ is the Jacobian of the convective flux and the time derivative of the variables in Eq. (4.60) is our IVP in Eq. (4.51). We use the scheme for the flux $\boldsymbol{F}$ instead of the variables $\boldsymbol{U}$. We do so because we can directly achieve the flux corrections in (4.58) by avoiding the calculation of the corrections in $\boldsymbol{U}$. From the space derivative of Eq. (4.51) we obtain by using Schwarz' theorem

$$
\begin{aligned}
\partial_{t} \boldsymbol{w}+\partial_{x} \boldsymbol{F}^{*} & =0 \\
\boldsymbol{w} & =\partial_{x} \boldsymbol{U} \\
\boldsymbol{F}^{*} & =\partial_{x} \boldsymbol{F}(\boldsymbol{U}(x, t))-\boldsymbol{S}(\boldsymbol{U}),
\end{aligned}
$$

which leads directly to the needed correction

$$
\partial_{t} \boldsymbol{F}=-\underline{\mathbf{J}} \cdot \boldsymbol{F}^{*}
$$

The problem (4.61) is a Riemann problem again, therefore we can use the MUSTA scheme again to solve it numerically. It is obvious that in principle it is possible to achieve higher order corrections in the same way as well. We stop after this correction in our solver because of performance issues and because the achieved solution $\boldsymbol{F}_{i+1 / 2}$ already leads to good results.

We still have to address the time integral of the source term. In principle there are again two ways of using the ADER scheme. The first way is to expand $\boldsymbol{U}(\tau)$ and calculate $\boldsymbol{S}(\boldsymbol{U}(\tau))$

$$
\begin{aligned}
\boldsymbol{U}(\tau) & =\boldsymbol{U}(0)+\tau \partial_{t} \boldsymbol{U}(0)+\mathcal{O}\left(\tau^{2}\right) \\
\boldsymbol{S} & =\boldsymbol{S}\left(\boldsymbol{U}(0)+\tau \partial_{t} \boldsymbol{U}(0)\right) \\
\boldsymbol{S} & =\boldsymbol{S}\left(\boldsymbol{U}(0)-\tau\left[\underline{\mathbf{J}} \partial_{x} \boldsymbol{U}-\boldsymbol{S}(\boldsymbol{U}(\mathbf{0}))\right]\right),
\end{aligned}
$$


and the second possibility is to correct the source term

$$
\begin{aligned}
\boldsymbol{S}(\tau) & =\boldsymbol{S}(0)+\tau \partial_{t} \boldsymbol{S}(0)+\mathcal{O}\left(\tau^{2}\right) \\
\partial_{t} \boldsymbol{S}(0) & =-\underline{\mathbf{J}_{\mathbf{S}}}\left(\partial_{x} \boldsymbol{F}(0)-\boldsymbol{S}(0)\right) \\
\underline{\mathbf{J}_{\mathbf{S}}} & =\nabla_{\boldsymbol{U}} \boldsymbol{S} .
\end{aligned}
$$

The source term is much easier to handle because no Riemann problem has to be solved. The reconstruction of the values at the Gaussian integration points is done via a 7th order WENO method. We implemented the version in Eq. (4.63). The Jacobian of the convective flux with respect to the conservative variables in our set of equations in (3.17) reads as

$$
\underline{\mathbf{J}}=\left(\begin{array}{ccccc}
0 & 1 & 0 & 0 & 0 \\
P-v_{x}^{2} & 2 v_{x}-\frac{2 v_{x} \partial_{T} p}{\rho} & -\frac{2 v_{y} \partial_{T} p}{\rho} & -\frac{2 v_{z} \partial_{T} p}{\rho} & \frac{\partial_{T} p}{\rho} \\
-v_{x} v_{y} & v_{y} & v_{x} & 0 & 0 \\
-v_{x} v_{z} & v_{z} & 0 & v_{x} & 0 \\
2 v_{x} P-\frac{v_{x}(2 p+\rho E)}{\rho} & \frac{2 p+\rho E}{\rho}-\frac{4 v_{x}^{2} \partial_{T} p}{\rho} & -\frac{4 v_{x} v_{y} \partial_{T} p}{\rho} & -\frac{4 v_{x} v_{z} \partial_{T} p}{\rho} & v_{x}\left(\frac{2 \partial_{T} p}{\rho}+1\right)
\end{array}\right),
$$

where $P=\left(\partial_{\rho} p+\frac{v^{2}-T}{\rho} \partial_{T} p\right)$. The eigenvalues of $\underline{\mathbf{J}}$ are $\lambda_{1}=\lambda_{2}=\lambda_{3}=v_{x}, \lambda_{4}=v_{x}-$ $c_{s}, \lambda_{5}=v_{x}+c_{s}$, where $c_{s}=\sqrt{\left(\frac{2 p}{d \rho^{2}} \partial_{T} p+\partial_{\rho} p\right)}$ is the speed of sound in the granular gas. The derivative of the pressure $p=p(\rho, T)$ with respect to $\rho$ and $T$ is calculated numerically by a fifth order central derivative

$$
\begin{aligned}
\partial_{\rho} p(\rho, T) & =\frac{-p(\rho[1+2 \epsilon], T)+8 p(\rho[1+\epsilon], T)-8 p(\rho[1-\epsilon], T)+p(\rho[1-2 \epsilon], T)}{12 \epsilon} \\
\partial_{T} p(\rho, T) & =\frac{-p(\rho, T[1+2 \epsilon])+8 p(\rho, T[1+\epsilon])-8 p(\rho, T[1-\epsilon])+p(\rho, T[1-2 \epsilon])}{12 \epsilon},
\end{aligned}
$$

where $\epsilon=10^{-5}$. Finally, we want to add that the ADER scheme naturally couples the source term and the convective flux via the correction terms.

\subsection{Courant-Friedrich-Lewy condition}

In the last sections we have described how to achieve a numerical convective flux on the surface of a finite volume. But we have not yet discussed the time step $\Delta t$ and how to choose it. We use the Courant-Friedrichs-Lewy (CFL) condition to calculate the step width $\Delta t$ of the convective flux

$$
\Delta t=C_{C F L} \frac{\operatorname{Max}(\Delta x, \Delta y, \Delta z)}{w_{\max }},
$$


where $w_{\max }$ is the maximum propagation speed of a convective wave and $C_{C F L}<1$ is the CFL number. The condition is achieved by the von Neumann stability analysis of the advection equation, more details to the stability analysis and the calculation can be found in [96]. From the point of view of the stability analysis the CFL condition is a necessary condition for the convergence of hyperbolic PDEs. But there is also quite a natural physical explanation of the CFL condition. The transport of information through

(a) $\frac{\Delta t}{w_{\max }} \geq \Delta x$

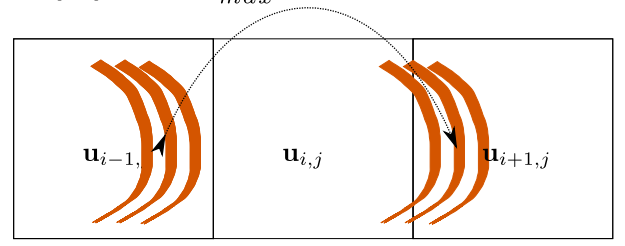

(b) $\frac{\Delta t}{w_{\max }}<\Delta x$

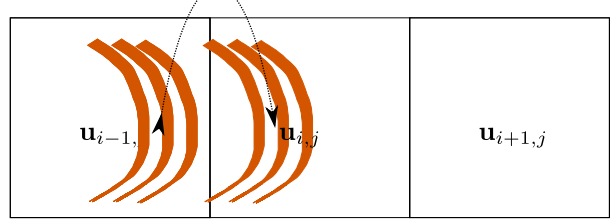

Figure 4.10: Wave propagation through finite volumes. In the time step $\Delta t$ the orange wave propagates by $\delta=\frac{\Delta t}{\omega_{\max }}$. Panel (a) shows the unphysical case when the wave propagation skips the volume $i$ during one time step. Panel (b) shows the necessary condition for stability, the propagation length $\delta$ has to be below $\Delta x$.

the system is limited by the fastest wave traveling with speed $w_{\max }$. In a small time $\Delta t$ the information can travel at most the distance $\delta=\frac{\Delta t}{w_{\max }}$. In our simulations the calculation domain is divided into small box. Figure 4.10 shows a sketch of a forbidden and a physical wave propagating through finite volumes. $\delta \geq \Delta x$ means now that the information transported with the wave never skips the finite volume $i$. That information is transported from box $(i-1)$ to box $(i+1)$ without being observed in box $i$ is unphysical. From these arguments immediately follows why $C_{C F L}$ must be smaller than one. In the case of $C_{C F L} \geq 1$ we observe an artificial coupling between the boxes $(i-1)$ and $(i+1)$. Typically we use a CFL number $C_{C F L}=0.2$ in our simulations. We want to add that the CFL condition is necessary, but may not be sufficient for the convergence of the schemes. Our simulations become unstable if dense regions form in the system. To further stabilize our simulation we decrease the CFL number if the system is not stable anymore (down to $C_{C F L} \approx 10^{-3}$ ).

\subsection{Diffusive flux}

Here we introduce the idea of the dGRP flux [97], which is a modification of the ideas of the Riemann problem to diffusive fluxes. Similar to the Riemann problem for convection (see Section 4.8) we consider the one dimensional IVP for scalar diffusion with a discontinuity at $x=0$

$$
\partial_{t} U-\kappa \partial_{x x} U=0
$$




$$
U(x, t=0)=\left\{\begin{array}{lll}
U^{L} & \text { if } & x<0 \\
U^{R} & \text { if } & x>0
\end{array}\right.
$$

with a constant $\kappa>0$. In the FVM (see Section 4.4) we can write the discretized solution at position $i$

$$
U_{i}^{n+1}=U_{i}^{n}+\frac{1}{\Delta x} \int_{t_{n}}^{t_{n}+1} \kappa \partial_{x} U\left(x_{i+1 / 2}, \tau\right) \mathrm{d} \tau-\frac{1}{\Delta x} \int_{t_{n}}^{t_{n+1}} \kappa \partial_{x} U\left(x_{i-1 / 2}, \tau\right) \mathrm{d} \tau
$$

The general solution of Eq. (4.68) reads as

$$
U(x, t)=\frac{1}{2 \sqrt{\kappa \pi t}} \int_{-\infty}^{\infty} U_{0}(\xi) e^{-\frac{(x-\xi)^{2}}{4 \kappa t}} \mathrm{~d} \xi .
$$

The derivative of the solution (4.71) using the piecewise constant initial data in Eq. (4.69) at $x=0$ is

$$
\partial_{x} U(0, t)=\frac{U^{R}-U^{L}}{2 \sqrt{\pi \kappa t}}
$$

We can now describe the numerical flux needed in the FVM in Eq. (4.70)

$$
\int_{t_{n}}^{t_{n}+1} \kappa \partial_{x} U\left(x_{i+1 / 2}, \tau\right) \mathrm{d} \tau \approx \kappa \Delta t \frac{U^{R}-U^{L}}{\sqrt{\pi \kappa t}} .
$$

This is only a legitimate approximation to Eq. (4.68) as long as

$$
\frac{1}{\sqrt{\pi \kappa \Delta t}}=\frac{1}{\Delta x}
$$

which in this case coincides with the central difference scheme. The generalization of the Riemann problem is to assume not a constant on the left and right side of the discontinuity but a polynomial expression of order $k^{3}$

$$
\begin{aligned}
& \partial_{t} U-\kappa \partial_{x x} U=0 \\
& U(x, 0)=\left\{\begin{array}{lll}
\sum_{j=0}^{k} c_{j}^{L} x^{j} & \text { if } & x<0 \\
\sum_{j=0}^{k} c_{j}^{R} x^{j} & \text { if } & x<0 .
\end{array}\right.
\end{aligned}
$$

The numerical flux can be written in this case as

$$
\begin{aligned}
& \int_{0}^{\Delta t} \kappa \partial_{x} U\left(x_{i+1 / 2}, \tau\right) \mathrm{d} \tau \\
& \approx \sum_{j=0}^{\lceil k / 2\rceil}\left(\left\{\partial_{x^{2 j}} U\left(x_{i+1 / 2}, 0\right)\right\}+\frac{4^{j}}{\sqrt{\pi \kappa \Delta t}}\left(\begin{array}{c}
2 j+1 \\
j
\end{array}\right) \llbracket \partial_{x^{2 j}} U\left(x_{x+1 / 2}, 0\right) \rrbracket\right) \frac{(\kappa \Delta t)^{j+1}}{(j+1) !}
\end{aligned}
$$

\footnotetext{
${ }^{3}$ For further details on generalized Riemann problems see [97, 98].
} 
with

$$
\begin{aligned}
\lceil k / 2\rceil & \equiv\left\{\begin{array}{cc}
k / 2 & \text { for } k \text { even, } \\
(k+1) / 2 & \text { for } k \text { odd }
\end{array}\right. \\
\{U\} & =1 / 2\left(U^{R}+U^{L}\right) \\
\llbracket U \rrbracket & =U^{R}-U^{L} \\
\left(\begin{array}{l}
n \\
k
\end{array}\right) & =\frac{n !}{k !(n-k) !}
\end{aligned}
$$

The details of how to achieve the approximation are found in the original paper [97]. The approximation in Eq. (4.76) allows to derive an approximation of any order $k$ in space-time of the numerical flux for the IVP in Eq. (4.68).

Now we consider the general nonlinear diffusion problem

$$
\begin{aligned}
& \partial_{t} \boldsymbol{U}-\partial_{x} \boldsymbol{F}\left(\boldsymbol{U}, \partial_{x} \boldsymbol{U}\right)=0 \\
& \boldsymbol{U}(x, 0)=\left\{\begin{array}{lll}
\boldsymbol{U}^{L} & \text { if } & x<0 \\
\boldsymbol{U}^{R} & \text { if } & x>0,
\end{array}\right.
\end{aligned}
$$

where $\boldsymbol{U}^{L}, \boldsymbol{U}^{R}$ are polynomials of order $k+1$. Then the numerical flux can be written as

$$
\boldsymbol{F}_{d G R P}(\tau)=\boldsymbol{F}\left(\left\{\boldsymbol{U}_{i+1 / 2}(\tau)\right\},\left\{\partial_{x} \boldsymbol{U}_{i+1 / 2}(\tau)\right\}+\frac{c(k)}{\sqrt{\pi} \Delta x} \llbracket\left\{\boldsymbol{U}_{i+1 / 2}(\tau)\right\} \rrbracket\right) .
$$

The lengthy details to achieve Eq. (4.79) are skipped and can be found in [97]. The time expansions $\boldsymbol{U}(\boldsymbol{\tau})$ can be obtained via the Cauchy-Kowalewski procedure. We implement a seventh order WENO method, for which the correction $c(k)=24.6$. The numbers $c(k)$ for different order $k$ can be found in [97]. The explicit time condition is

$$
\Delta t \leq \frac{\Delta x^{2}}{\lambda_{\max } d^{2}}
$$

where $\lambda_{\max }$ is the maximum eigenvalue of the matrix of coefficients of the diffusive flux 4. The matrix of coefficients of the diffusive flux with respect to the primitive variables in the $x$-direction for our set of equations in (3.17) is

$$
\underline{\mathbf{D}}_{x}^{p}(\boldsymbol{U})=\frac{1}{\mathcal{R}}\left(\begin{array}{ccccc}
0 & 0 & 0 & 0 & 0 \\
0 & 4 / 3 & 1 & 1 & 0 \\
0 & -2 / 3 & 1 & 0 & 0 \\
0 & -2 / 3 & 0 & 1 & 0 \\
\frac{\mathcal{C}_{\mu}}{\mathcal{P}} & 4 / 3 v_{x}-2 / 3 v_{y}-2 / 3 v_{z} & v_{x}+v_{y} & v_{x}+v_{z} & \frac{1}{\mathcal{P}}
\end{array}\right) \text {. }
$$

\footnotetext{
${ }^{4}$ The global time step is the minimum of the convective condition (see Section 4.13) and the diffusive time-step condition.
} 
We get the matrix of coefficients with respect to conservative variables by the use of the chain rule

$$
\underline{\mathbf{D}}_{x}(\boldsymbol{U})=\underline{\mathbf{D}}_{x}^{p}(\boldsymbol{U}) \cdot \nabla_{U}\left(\begin{array}{c}
\rho \\
\boldsymbol{v} \\
T
\end{array}\right)
$$

$\underline{\mathbf{D}}_{x}$ has the nonvanishing eigenvalues $\lambda_{1}=\frac{2}{3 \mathcal{R} \mathcal{P} \rho}, \lambda_{2}=\frac{1}{\mathcal{R} \rho}, \lambda_{3,4}=\frac{1}{6 \mathcal{R} \mathcal{P}}(7 \pm \mathrm{i} \sqrt{47})$. The matrices of coefficients for the $y$ - and $z$-direction follow out of symmetry.

The dependence of $\Delta t$ on $\Delta x^{2}$ is typical for diffusive conditions and may lead to really small steps. Explicit schemes as presented here are local schemes, which are well suited for an easy implementation in CUDA. Sadly it turns out that the time-step condition in Eq. (4.80) drastically reduces the time-step especially in driven systems. The alternative implicit methods do not have a time step condition and may look more useful for a diffusive flux but implicit methods couple the calculation cells in the entire calculation domain with each other which is a challenging task for parallelization with CUDA. One of our future projects for further development of the DNS will contain an implementation of an implicit solver for the diffusive flux. Finally, we want to add that the dGRP flux drastically decreases the performance of our solver (by a factor $\approx 3$ ) and for this reason we use whenever possible a central flux method as a fast alternative, which leads to qualitatively the same results. The central flux methods corresponds to setting $c(k)=0$ in Eq. (4.79).

\subsection{Boundary conditions}

We study systems with different boundary conditions. Periodic boundaries are often chosen to minimize the effects of boundaries, although they represent a highly theoretical situation with nearly no experimental matches. For other simulations it is even impossible to neglect the effects of boundaries. One example are systems where we inject energy via the shaking of confining, solid walls. We want the solver to be written in a way that it is easy to implement or change boundary conditions without much effort. In addition, it is important for the CUDA implementation that we are able to use the same methods everywhere inside our calculation domain. A good way to implement boundary conditions under such requirements is the use of ghost cells. The concept of ghost cells means that around the calculation domain additional cells are introduced and their values are set instead of being calculated. Figure 4.11 shows the concept of the ghost cell for a simulation domain of arbitrary shape. By setting the values of the cells inside the boundary all kinds of boundary conditions can be achieved. It must be ensured that the data are written to the ghost cells before they are needed by any method. For our specific implementation, we need four ghost cells to each side because we implemented a seventh order WENO method which needs 4 data points to the left and the right from the position $i$ where it is used. 


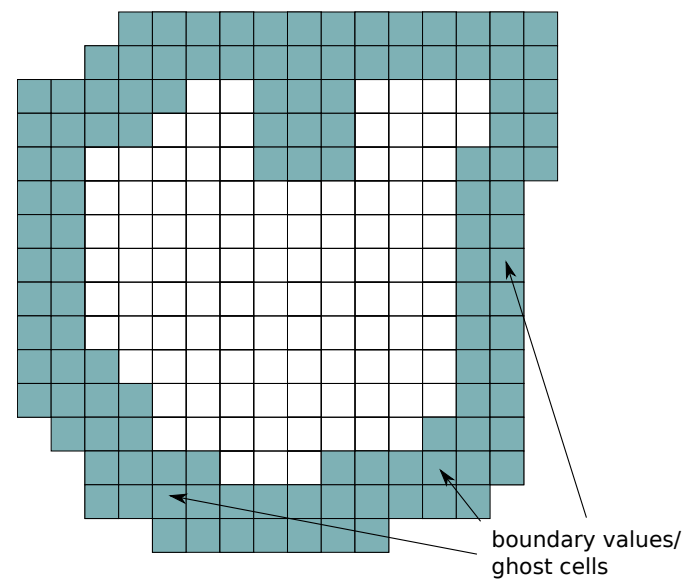

Figure 4.11: A sketch of a 2D system with two ghost cells at each boundary location. The values of the ghost cells must be set to satisfy the needed boundary conditions.

We briefly describe below how to set the different conditions.

We assume a system of size $N_{X} \times N_{Y} \times N_{Z}$, the calculation domain is described by the indices $i=0,1, \ldots,\left(N_{X}-1\right), \quad j=0,1, \ldots,\left(N_{Y}-1\right), \quad k=0,1, \ldots,\left(N_{Z}-1\right)$. Each index has four more values to the left $(-4,-3,-2,-1)$ and to the right $\left(N_{X}, N_{X}+1, N_{X}+\right.$ $\left.2, N_{X}+3\right)$, which represent the ghost volumes and for which we have to set the values $\underline{\mathbf{W}}=(\rho, \underline{\mathbf{v}}, T)$. Periodic boundary condition are achieved just by copying the data from the opposite side. For example, in $x$-direction

$$
W_{i}=\left\{\begin{array}{llc}
W_{\left(i+N_{X}\right)}, & \text { if } & i<0 \\
W_{\left(i-N_{X}\right)}, & \text { if } & i \geq N_{X}
\end{array}\right.
$$

To model adiabatic walls we set the ghost volume to be a mirror of the fields at the boundaries, for the $x$-dimension ( $y$ and $z$ analogues) we find

$$
\begin{aligned}
\rho_{i} & =\left\{\begin{array}{ccc}
\rho_{(-i-1)}, & \text { if } & i<0, \\
\rho_{\left(2 N_{X}-1-i\right)}, & \text { if } & i \geq N_{X}
\end{array}\right. \\
\left(v_{x}\right)_{i} & =\left\{\begin{array}{ccc}
-\left(v_{x}\right)_{(-i-1)}, & \text { if } & i<0, \\
-\left(v_{x}\right)_{\left(2 N_{X}-1-i\right)}, & \text { if } & i \geq N_{X}
\end{array}\right. \\
\left(v_{y}\right)_{i} & =\left\{\begin{array}{ccc}
C_{f}\left(v_{y}\right)_{(-i-1)}, & \text { if } & i<0, \\
C_{f}\left(v_{y}\right)_{\left(2 N_{X}-1-i\right)}, & \text { if } & i \geq N_{X}
\end{array}\right. \\
\left(v_{z}\right)_{i} & =\left\{\begin{array}{ccc}
C_{f}\left(v_{z}\right)_{(-i-1)}, & \text { if } \quad i<0, \\
C_{f}\left(v_{z}\right)_{\left(2 N_{X}-1-i\right)}, & \text { if } \quad i \geq N_{X}
\end{array}\right. \\
(T)_{i} & =\left\{\begin{array}{ccc}
(T)_{(-i-1)}, & \text { if } & i<0, \\
(T)_{\left(2 N_{X}-1-i\right)}, & \text { if } & i \geq N_{X},
\end{array}\right.
\end{aligned}
$$

where $C_{f} \in[-1,1]$ is the strength of the friction at the boundary. Thermal heating through the boundary can be achieved by setting the temperature of the ghost cell in (4.84), even if it might not be clear what thermal heating means in the context of granular gases. The 
third important boundary condition we implemented are open boundaries which are set in a way that the derivatives vanish at the boundary

$$
\begin{aligned}
& \rho_{i}=\left\{\begin{array}{ccc}
\rho_{0}, & \text { if } \quad i<0, \\
\rho_{N_{X}-1}, & \text { if } \quad i \geq N_{X}
\end{array}\right. \\
& \left(v_{x}\right)_{i}=\left\{\begin{array}{ccc}
-\left(v_{x}\right)_{0}, & \text { if } \quad i<0, \\
-\left(v_{x}\right)_{N_{X}-1}, & \text { if } \quad i \geq N_{X}
\end{array}\right. \\
& \left(v_{y}\right)_{i}=\left\{\begin{array}{ccc}
C_{f}\left(v_{y}\right)_{0}, & \text { if } \quad i<0, \\
C_{f}\left(v_{y}\right)_{N_{X}-1}, & \text { if } \quad i \geq N_{X}
\end{array}\right. \\
& \left(v_{z}\right)_{i}=\left\{\begin{array}{ccc}
C_{f}\left(v_{z}\right)_{0}, & \text { if } \quad i<0, \\
C_{f}\left(v_{z}\right)_{N_{X}-1}, & \text { if } \quad i \geq N_{X}
\end{array}\right. \\
& (T)_{i}=\left\{\begin{array}{ccc}
(T)_{0}, & \text { if } \quad i<0, \\
(T)_{N_{X}-1}, & \text { if } \quad i \geq N_{X} .
\end{array}\right.
\end{aligned}
$$

The concept of ghost cells has the advantage that it is a simple and flexible method adaptable to all kinds of boundary conditions. A disadvantage is the additional memory which is needed to store the ghost cells and the additional calculations which have to be done by setting their values. In our case about $10 \%$ of the calculation time went into the setting of the ghost volumes.

\subsection{Initialization}

To solve the IVP in (3.17) we have to initialize our simulations with initial values $\boldsymbol{U}_{i}^{0}$. In our simulation we set the initial values for the density $\bar{\rho}_{i}$, velocity $\overline{\boldsymbol{v}}_{i}$ and the temperature $\bar{T}_{i}$ for each finite volume $i$, all other variables like pressure, energy and momentum are calculated out of this quantities. Computationally there are problems with the handling of exactly equal values in the quantities, mainly because differences are not well defined (typical they are not zero but a small value of the order of numerical precision). Therefore we have to add white noise on each quantity. Typical we use a noise strength of the $\mathcal{O}\left(10^{-8}\right)$. In the case of running simulations including external forces, we use a transient time to turn on all external forces. This has to be done so that the system can adapt to the external forces, otherwise we would have to deal with strong shocks in the initial data, which is in most cases an unsurmountable problem for a numerical solver. The transient effects are modeled by a third order polynomial with a turning point of value zero at time zero and a second turning point of value one when the time equals the transient time. We tested in free cooling simulation the effect of an initial equilibration of the system by setting $\varepsilon=1$ for $t=10^{4} \frac{\sigma}{v_{t h, 0}}$. We could not measure a different behavior from a system without initial equilibration. 


\section{A universal scaling law for the evolution of granular gases}

Dry, freely evolving granular materials in a dilute gaseous state coalesce into dense clusters only due to dissipative interactions. In this chapter we show that the evolution of a dilute, freely cooling granular gas is determined in a universal way by the ratio of inertial flow and thermal velocities, that is, the Mach number. We give an analytical argument to support our findings and finally we discuss the scaling of the clusters in a freely cooling granular gas. We found out that clusters form on a characteristic length scale which is a crucial parameter for the setup of the system and to obtain a good resolution without measuring finite size effects.

\subsection{The global Mach number as an indicator for clustering}

A freely cooling granular gas is an ensemble of particles undergoing dissipative collisions and free of any external field. Even a small degree of dissipation in the kinetics of granular particles produces spatial correlations and structures in a dilute, homogeneous gas [34]. Hydrodynamic treatments suggest that a shear instability initiates this transition [34] (see the linear stability analysis in Section 2.9). However, when exactly this process initiates is not known.

The equations of granular hydrodynamics in Eq. (3.17) predict a linear instability of the transverse mode [35] when the wavevector $\|\boldsymbol{k}\| \leq k_{\perp}^{*}(\varepsilon)$, where $k_{\perp}^{*}(\varepsilon) \propto\left(1-\varepsilon^{2}\right)^{1 / 2}$ (see also Section 2.9). This instability leads to the formation of vortices. In regions where the particle velocities are correlated the temperature drops. These are the seeds for a 


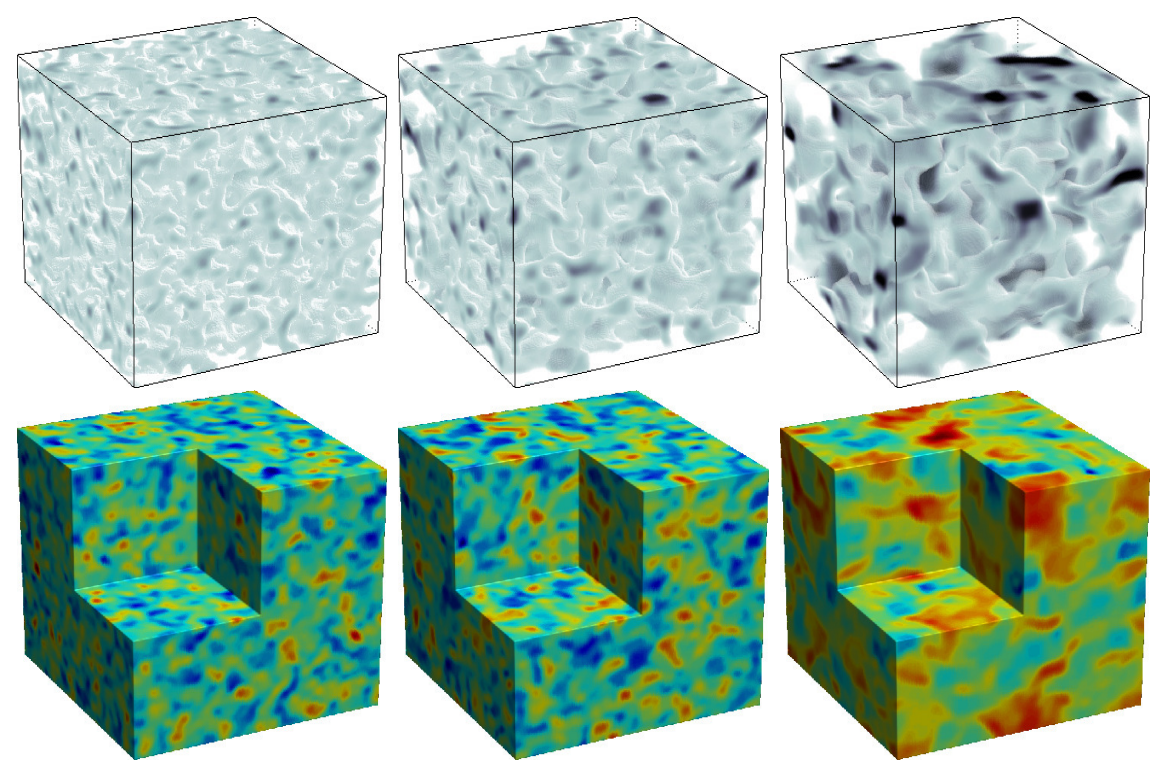

Figure 5.1: Snapshots of the system showing the temporal evolution of a three-dimensional granular gas with a coefficient of restitution $\varepsilon=0.9$ and average filling fraction $\bar{\phi}=0.05$. (Top row) From left to right, snapshots of the density field at times $t=10^{9}, t=10^{10}$ and $t=10^{11}$, respectively. The gray scale represents the local average density. (Bottom row) Three-dimensional map of the temperature field $T(\boldsymbol{r}, t)$ at the same times of the corresponding density plot in the top row. The color (red - hot; blue - cold) represents the local temperature compared to the average temperature in the system.

second instability if the system size is larger than $k_{\mathrm{H}}^{*-1}(\varepsilon)>k_{\perp}^{*-1}(\varepsilon)$. Although freely cooling granular gases have attracted wide interest [21-23, 36, 99-101] there remains, beyond issues of finite sizes, the outstanding question of what sets the rise of the density inhomogeneities. Past works made differing claims as to what the onset time of clustering is $[36,100,102]$.

The simulations in this section are performed in the HCS, therefore we did not use the term for the bulk viscosity in the hydrodynamic equations in Eq. (3.17) and we use the Carnahan-Starling pair-correlation function in Eq. $(3.1)^{1}$.

Figure 5.1 shows the evolution of $\rho(\vec{r}, t)$ and $T(\vec{r}, t)$ in a freely cooling gas in 3D. We observe that out of the homogeneous cooling state small, cold regions of larger density emerge throughout the system and grow in size with time. The high density regions (clusters) are filamentous because of the shear instability. The morphology of the clusters that we find in our simulations agrees with the shape of the clusters which have been seen in MD simulations [24, 104], however, our system size allows the formation of multiple, large clusters (see also the attached movie 3Dcooling.avi). We quantify the cluster growth by calculating the maximum filling fraction $\phi_{\max }$ and the density fluctuations $\left\langle(\delta \rho)^{2}\right\rangle=$

\footnotetext{
${ }^{1}$ All simulations are stopped once the maximum local filling fraction $\phi_{\max }$ is $5 \%$ above average filling fraction $\bar{\phi}$; this ensures that our calculations remain in a regime where the pair correlation function holds. $\phi_{\max }$ is the maximum value of all local filling fractions $\phi_{i}$ in the system. The results of this work are valid till the point when the system starts forming dense clusters. For further discussion on the validity and limits of the equations of state see [103].
} 

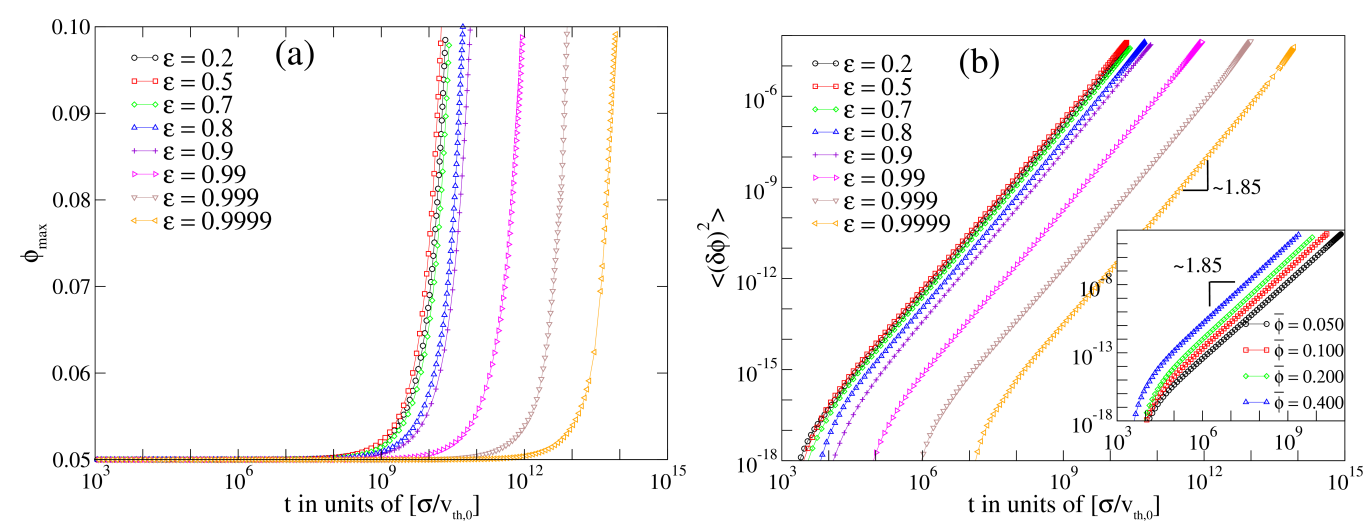

Figure 5.2: Temporal evolution of the maximum filling fraction $\phi_{\max }$ (a) and of the filling fraction fluctuations $\left\langle(\delta \phi)^{2}\right\rangle$ (b). In (a) and in the main panel of (b) the average filling fraction $\bar{\phi}=0.05$. The time is measured in units of $\sigma$ over the initial thermal velocity $v_{t h, 0}$. The clustering process exhibits the same qualitative features over a wide range of coefficients of restitution $\varepsilon$ and average filling fractions $\bar{\phi}$. The curves are guides to the eye. As $\varepsilon$ grows, the time of onset of clustering increases of four orders of magnitude. The inset panel shows the effect of varying average filling fractions. The initial crossover at $\left\langle(\delta \phi)^{2}\right\rangle \approx 10^{-16}$ is an effect of the initial transient.

$\frac{1}{\bar{\phi}^{2}}\left\langle(\delta \phi)^{2}\right\rangle$ across the system, which allows one to compare systems with different average filling fractions $\bar{\phi}$. Hereafter the angle brackets indicate spatial averaging over the entire system. Figure 5.2 shows the temporal evolution of these quantities. In the homogeneous cooling state $\phi_{\max }$ is very small, but suddenly the systems develops inhomogeneities. Figure 5.2(b) reveals an early time dynamics not directly accessible when observing the maximum or the average filling fraction (Fig. 5.2(a)). What appears to be a sudden emergence of clusters at characteristic times depending on $\varepsilon$ in Fig. 5.2(a) is instead a scalefree process described by a power law $\sim t^{\alpha}$, with $\alpha \approx 1.85$. Thus, if the signal-to-noise ratio of the measurements is not large enough an apparent sudden onset of clustering will be visible only when $\phi_{\max }$ has reached few percent. To understand the origin of the increase of the density we examine the behavior of the average kinetic energy $E_{k i n} \equiv \sum_{i} \rho_{i} v_{i}{ }^{2} / \sum_{i} \rho_{i}$ and the average temperature $T \equiv \sum_{i} \rho_{i} T_{i} / \sum_{i} \rho_{i}$, where $i$ is the index of the finite volumes, and compare it with the temporal evolution of $\left\langle(\delta \phi)^{2}\right\rangle$. The temperature follows Haff's law $T(t)=T_{0}(1+t / \tau)^{-2}, \tau \propto \bar{\phi}\left(1-\varepsilon^{2}\right) \sqrt{T_{0}}$, while $E_{K i n}(t)$ decays with time in a much weaker way. Figure 5.3 shows that the size of the inhomogeneities is directly linked to the relative decrease of the kinetic energy $E_{K i n}(t)$ with respect to the temperature. In fact, when the kinetic energy equals the temperature (Haff's law), $\left\langle(\delta \phi)^{2}\right\rangle$ reaches the same value $\approx 10^{-4}$ for all our simulations. This point coincides with the time when clusters would become visible in an experiment or molecular dynamic simulation (i.e. $\Delta \phi \sim \sqrt{\left\langle(\delta \phi)^{2}\right\rangle} \approx 1 \%$. Therefore, it seems appropriate to us to consider the Mach number $\mathcal{M} \equiv \sqrt{\left\langle v^{2}\right\rangle /\langle T\rangle}$ as a scaled measure of the granular dynamics. The granular gas develops visible clusters when $\mathcal{M}$ is of the order of unity, that is the threshold of supersonic flow. Changing the average filling fraction or coefficient of restitution does not alter this 


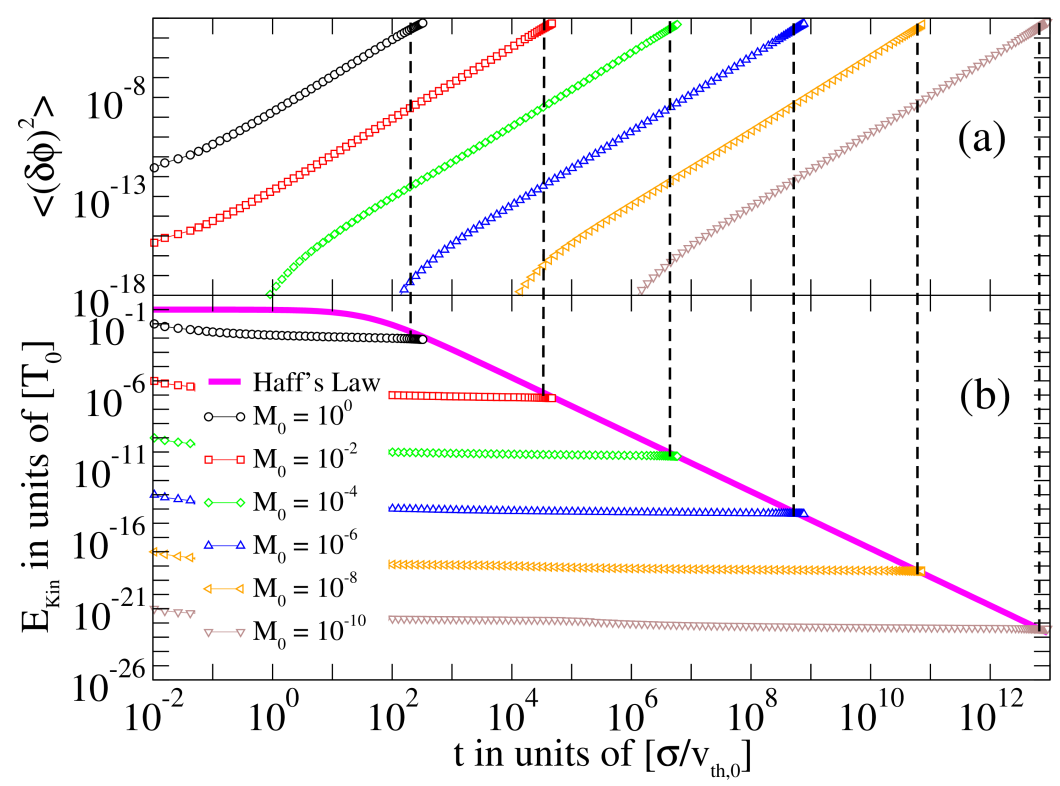

Figure 5.3: (a) Temporal evolution of density fluctuations for different initial values of the Mach number $\mathcal{M}_{0}$. (b) Evolution of the kinetic energy (symbols) for the same initial Mach numbers as in (a), and evolution of the temperature (solid, magenta line). We mark the times $t^{*}\left(\mathcal{M}_{0}\right)$ when the kinetic energy equals the temperature and the corresponding values of $\left\langle(\delta \phi)^{2}\right\rangle\left(t^{*}\right)$ (vertical, dotted lines). To test the dependence of the density fluctuations on $\mathcal{M}$, we vary the initial, inertial velocities in the system, that is, the initial value of the Mach number $\mathcal{M}_{0}$. We find that for larger $\mathcal{M}_{0}$ the onset of clustering occurs decades in time earlier, but still it coincides with the time when $\mathcal{M} \sim \mathcal{O}(1)$. 


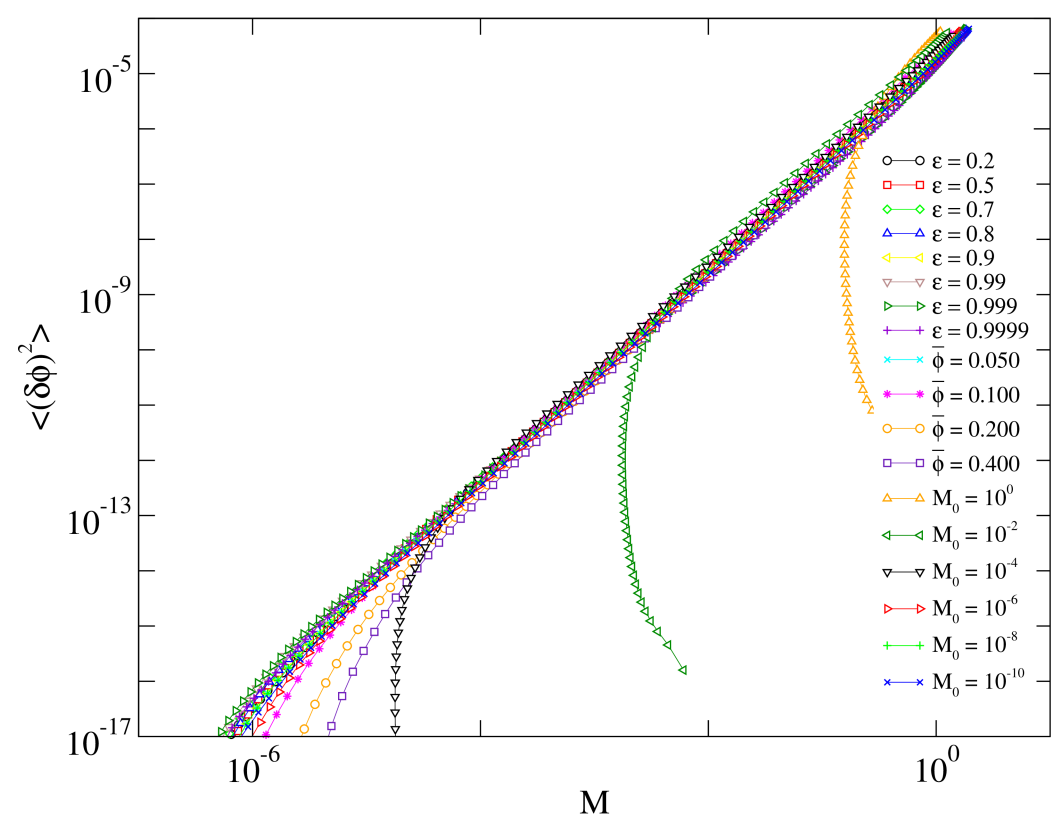

Figure 5.4: The density fluctuations exhibit a universal scaling in terms of the system's average Mach number $\mathcal{M}$. After the relaxation of the initial conditions the granular gas shows a collapse of the density fluctuations on the curve $\left\langle\delta \rho^{2}\right\rangle(\mathcal{M})=c \mathcal{M}^{2}$. Calculations are shown for system at $\bar{\phi}=0.05, \varepsilon=0.9$ and $\mathcal{M}_{0}=10^{-8}$ unless the variable are explicitly changed according to the legend.

conclusion. We note that Haff's law is derived under the assumption of no macroscopic fluxes and homogeneous density [19] (see Section: 2.8). Because of these assumptions it is not possible to conclude anything about the density nor the velocity fluctuations out of Haff's law. In the context of our definition of the Mach number Haff's law assumes $\mathcal{M}=0$.

Because the system is translational invariant and there is no characteristic time scale associated with the condition $\mathcal{M} \sim \mathcal{O}(1)$ we expect the evolution of the freely cooling gas to be scale invariant when described with the relevant variables.

Figure 5.4 collects results from extensive DNS calculations where we varied $\varepsilon, \mathcal{M}_{0}, \bar{\phi}$ and shows the evolution of $\left\langle(\delta \phi)^{2}\right\rangle$ in terms $\mathcal{M}$. Regardless of the system parameters or the initial state, the density fluctuations converge onto the locus $\left\langle(\delta \phi)^{2}\right\rangle(\mathcal{M})=c \mathcal{M}^{2}$, where $c$ is a constant. This universal scaling is robust also upon variation of the initial conditions. Figure 5.5 plots different simulation with the same system parameters, but different fluctuations in the initial density and temperature fluctuations. Furthermore we observe the same scaling behavior for two dimensional systems with constant and variable ${ }^{2}$ coefficient of restitution. Figure 5.6 displays the results of the viscoelastic sphere model in $3 \mathrm{D}$.

This locus $\left\langle(\delta \phi)^{2}\right\rangle=c \mathcal{M}^{2}$ plays the role of an "attractor" for the evolution of the granular gas, as it is visible from Fig. 5.4: when the initial Mach number $\mathcal{M}_{0}$ is large, the

\footnotetext{
${ }^{2}$ We use the viscoelastic spheres model described in Section 2.2 to simulate a variable coefficient of restitution. For implementation issues see [105].
} 


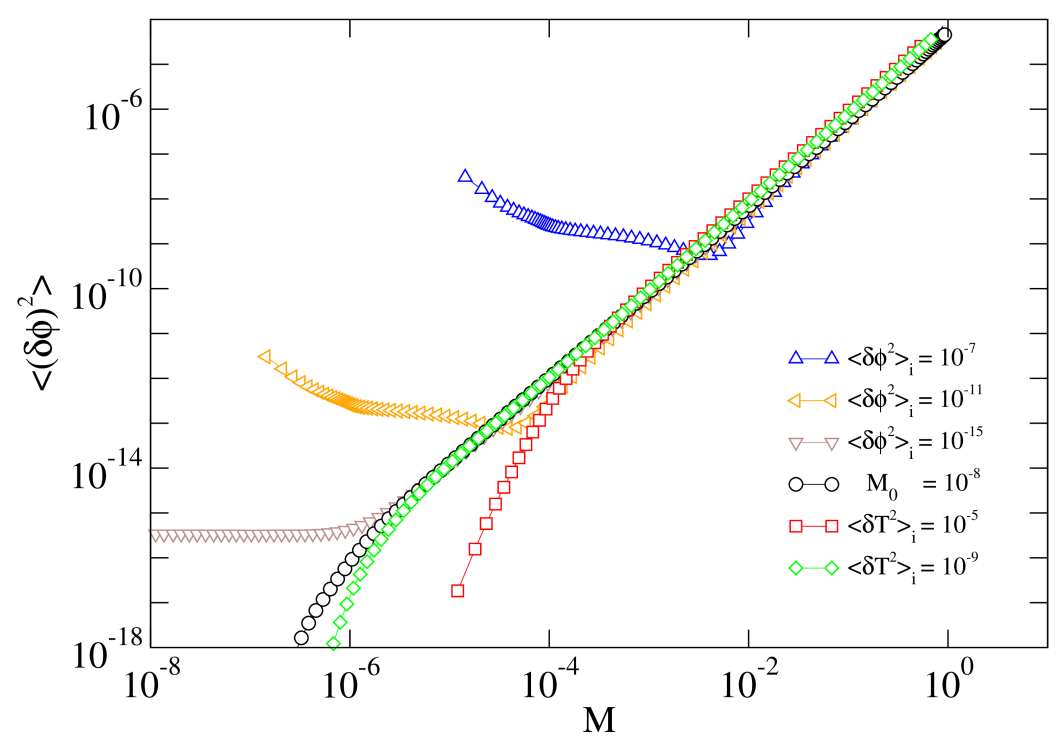

Figure 5.5: Free cooling systems with different initial fluctuation in the density and temperature. The system parameters are $\bar{\phi}=0.05, \varepsilon=0.9$ and $\mathcal{M}_{0}=10^{-8}$. The figure displays that after a certain time the initial conditions are smoothed out.

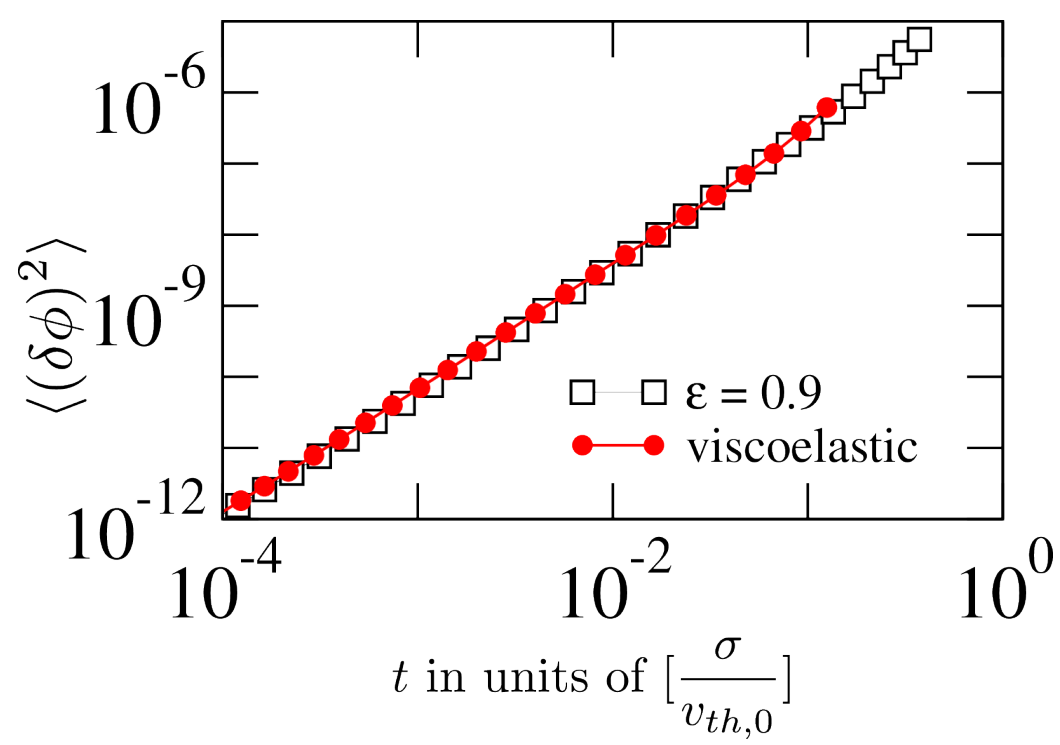

Figure 5.6: Density fluctuation for a model of viscoelastic spheres and const $\varepsilon$ in 3D. Both models follow the same scaling behavior. 
system shows a weak transient decrease of $\mathcal{M}_{0}$, due to a relaxation of the initial condition, which is then followed by the approach and collapse onto the locus $\left\langle(\delta \phi)^{2}\right\rangle=c \mathcal{M}^{2}$. Once the initial conditions are forgotten, all systems investigated show universal behavior, as visible from the collapse of all curves in Fig. 5.4 over several decades. The universal powerlaw behavior of a granular gas in the homogeneous cooling state indicates that there is no characteristic time scale for the onset of clustering. However, because of finite resolution, an experiment would observe the onset of clustering when $\mathcal{M} \sim \mathcal{O}(1)$.

The results of this section might provide a tool to measure the age of such granular gases in the context of the Mach number. In summary, we have shown by means of DNS that $(i)$ the dynamics of a freely cooling granular gas follows a universal power-law behavior in terms of the ratio of convective to thermal velocities, irrespective of the initial state, and (ii) the onset of clustering has no characteristic time scale.

\subsection{Heuristic for the universal scaling}

We now want to show that under general assumptions we can prove with an analytical argument that the density fluctuations $\left\langle(\delta \rho)^{2}\right\rangle$ scale quadratically with $\mathcal{M}$ as we found in Section 5.4. We assume that: $(i)$ the granular gas is in a homogeneous cooling state where Haff's law holds, $T=T_{0}(1+t / \tau)^{-2}$, and the density fluctuations are still small, that is, $\rho=\rho_{0}+\delta \rho, \delta \rho \ll \rho_{0}$; (ii) isotropy of the velocity, $\left\langle v_{x}\right\rangle=\left\langle v_{y}\right\rangle=\left\langle v_{z}\right\rangle$; (iii) the equation of state of ideal gases holds: $p=\rho T$; $(i v)$ the system is so large that we can neglect finite size effects and that diffusion is negligible; $(v)$ local convective fluxes and their gradients are small: $v_{i}, \partial_{i} v_{j} \ll T^{1 / 2}, i, j \in\{x, y, z\}$. Equations (3.17) then become

$$
\begin{aligned}
\partial_{t} \rho+\nabla \cdot(\rho \boldsymbol{v}) & =0 \\
\partial_{t} \boldsymbol{v}+\frac{1}{\rho} \nabla(\rho T) & =0 .
\end{aligned}
$$

We start by multiplying Eq. (5.1) by $\rho^{-1}$ and taking the spatial average over the volume $\Omega,\langle\Phi\rangle=\frac{1}{|\Omega|} \int_{\Omega} \Phi d \Omega$. We consider fluctuations around a background solution, so that we can write

$$
\begin{aligned}
\partial_{t}\langle\log (\rho)\rangle & =\partial_{t}\left\langle\log \left(\rho_{0}\right)\right\rangle+\partial_{t}\langle\delta \rho\rangle / \rho_{0}-\frac{1}{2} \partial_{t}\left\langle\delta \rho^{2}\right\rangle \rho_{0}^{2}+\mathcal{O}\left(\delta \rho^{3} / \rho_{0}^{3}\right) \\
& =-\frac{1}{2} \partial_{t}\left\langle\delta \rho^{2}\right\rangle / \rho_{0}^{2}+\mathcal{O}\left(\delta \rho^{3} / \rho_{0}^{3}\right)
\end{aligned}
$$

where $\rho_{0}=\langle\rho\rangle$ is the average density. The surface integral $\langle\nabla \cdot v\rangle$ vanishes. Using Eq. (5.3), Eq. (5.1) becomes

$$
-\frac{1}{2} \partial_{t}\left\langle\delta \rho^{2}\right\rangle / \rho_{0}^{2}+\left\langle\frac{\boldsymbol{v}}{\rho} \cdot \nabla \rho\right\rangle=0
$$


Equation (5.2) is multiplied by $v$, followed by the space averaging

$$
\frac{1}{2} \partial_{t}\left\langle v^{2}\right\rangle+T\left\langle\frac{\boldsymbol{v}}{\rho} \cdot \nabla \rho\right\rangle=0
$$

where we assumed that the spatial variation of $T$ is negligible. Equations (5.4) and (5.5) lead to

$$
\left\langle\delta \rho^{2}\right\rangle=-\int \frac{\partial_{t}\left\langle v^{2}\right\rangle}{T} d t+\text { const }
$$

Haff's law predicts that $T=T_{0}\left(\tau^{-1} t\right)^{-2}$ for $t \gg \tau$, which we insert in Eq. (5.6). From our simulations we find out that the kinetic energy follows also a power law $\frac{1}{2}\left\langle v^{2}\right\rangle \propto t^{-A}$, $A \approx 0.07$ (see Fig. 5.3a). Combining this result with Eq. (5.6) we find

$$
\left\langle\delta \rho^{2}\right\rangle / \rho_{0}^{2} \propto \frac{A}{2-A} \mathcal{M}^{2}+\text { const. }
$$

In summary, with rather mild assumptions on the granular dynamics we can predict that the density fluctuations scale quadratically with the Mach number of the gas.

\subsection{Correlation of density and temperature in the homogeneous cooling state}

During the cooling of a granular gas, local fluctuations in the density will lead to a regime of increased density. In these high density regions more collisions occur and therefore the temperature should decrease locally. In Fig. 5.7(a) we show again the results of 3D simulations. Already visual inspection reveals that the temperature is lower wherever the density is large. Figure 5.7 shows the correlation between density and temperature for a freely cooling granular gas. We measure a nearly total anti-correlation between temperature and density in the system. This result agrees with the expected behavior and match the results from MD simulations [22]. In Fig. 5.7(c) we plotted the correlation coefficient

$$
\operatorname{Cor}(\rho, T)=\frac{\langle(\rho-\langle\rho\rangle)(T-\langle T\rangle)\rangle}{\sqrt{\left\langle(\delta \rho)^{2}\right\rangle} \sqrt{\left\langle(\delta T)^{2}\right\rangle}}
$$

Apart from transient effects, we can observe a total anti-correlation of the density and the temperature in the entire HCS of the granular gas. Interestingly, during the transition from the HCS to the ICS we find that the anti-correlation vanishes. Even if this behavior has not been studied in full detail so far we see the reason in uprising shock waves, which we study in detail in Section 6 . 

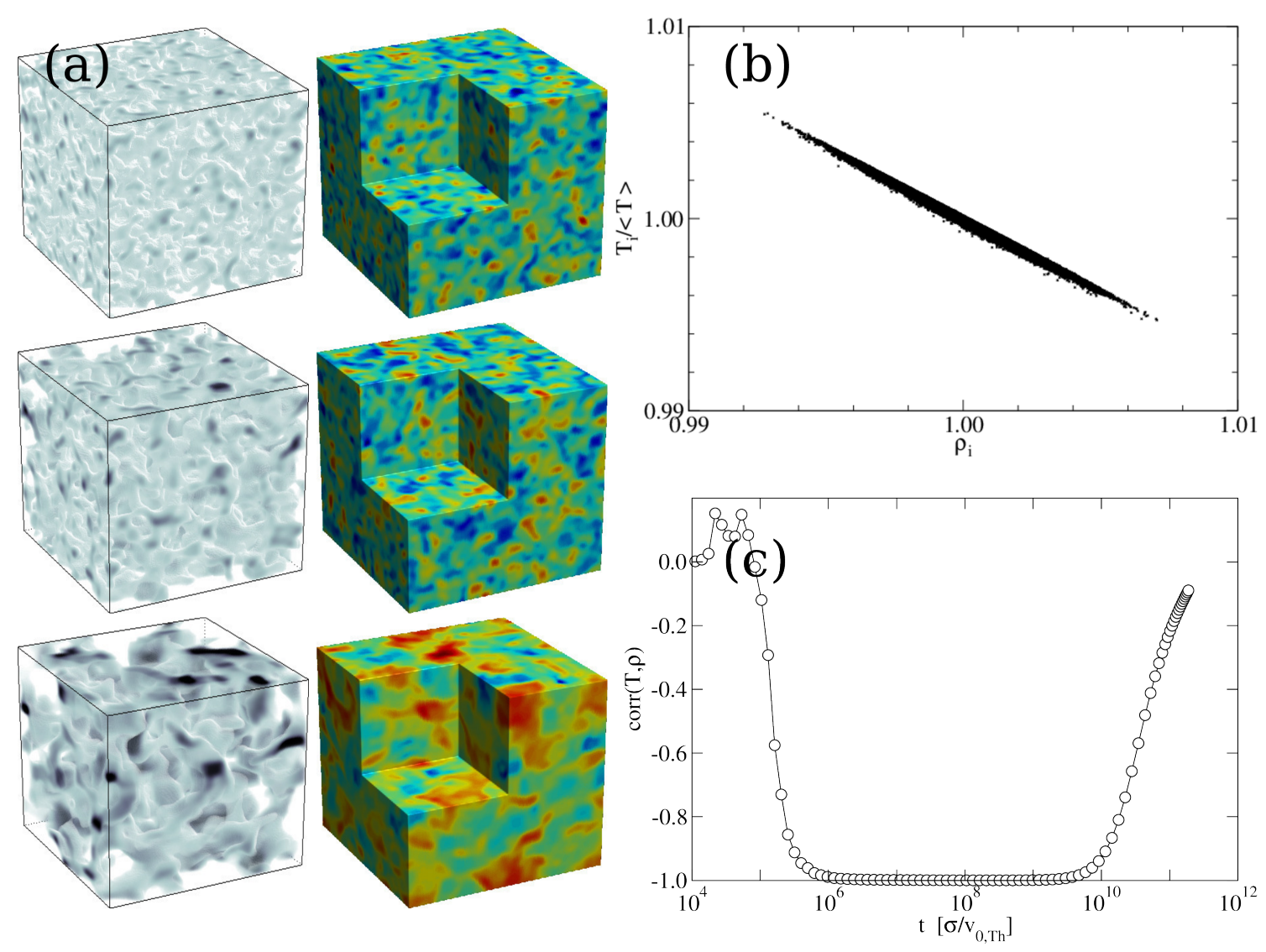

Figure 5.7: Panel (a) shows the temperature and density at different times in the HCS, panel (b) shows their correlation in the last figure of panel (a). Panel (c) plots the temporal evolution of the correlation coefficient $\operatorname{Cor}(\rho, T)$ during the entire HCS (the transition to the ICS appears in the region of $t=10^{10}$ ). (The data are the same as in Fig. 5.1.) 


\subsection{Scaling issues}

We now want to discuss the problems associated with the scaling of the system in our simulations. This can indeed become an important issue when studying the clustering in freely cooling granular gases. The resolution with which we can calculate physical observables is limited by the number of finite volumes of our DNS calculations. This is typically set to $1024^{2}$ in $2 \mathrm{D}$ and $128^{3}$ in $3 \mathrm{D}$. If we choose system sizes which are too small we observe finite size effects due to the boundaries or in the case of periodic boundaries we observe self-interaction of the density inhomogeneities which eventually creates waves in the system. On the other hand, we cannot choose an arbitrary large system because the finite volumes would correspond to regions so large that the physical fields do not carry relevant information any longer. Technical limitations of the current hardware restrict the range of system sizes we can usefully investigate.
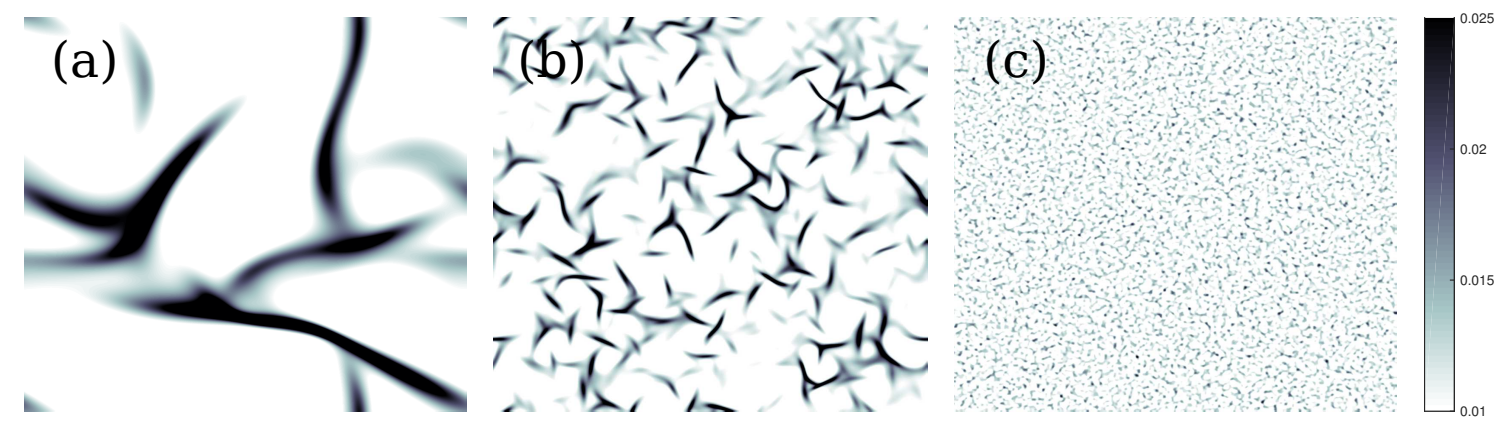

Figure 5.8: Density field of a freely cooling system at different system sizes. (a) $L_{\text {sys }}=$ $5120 \sigma$, (b) $L_{\text {sys }}=256000 \sigma$, (c) $L_{\text {sys }}=512000 \sigma$

Figure 5.8 shows the density field at different resolutions for three freely cooling systems with the same physical parameters but which differ only in size. Panel (a) shows high selfinteraction of the clusters; panel (c) corresponds to a size so large that one cannot resolve the filamentary shape of the clusters, whereas Fig. 5.8(b) shows. Figure 5.8 shows that at least during the formation of dense clusters, these clusters have a typically size. Sadly we do not know very much about the long time evolution of the clusters in the ICS because the simulations become unstable at some point.

We choose in our simulation system sizes which allow the formation of multiple clusters as in Fig. 5.8(b). Nevertheless we figured out that the results presented in Section 5.1 are still valid even for really small systems and weak resolutions.

The problem of finding appropriate resolution and system size is even more complicated in $3 \mathrm{D}$ because the number of finite volumes, which contain the data, are even smaller in each direction in 3D. We found empirically that an appropriate system size that excludes finite-size effects in $3 \mathrm{D}$ ranges from $L_{\text {sys }}=128 \times 10^{2} \sigma$ for $\varepsilon \leq 0.90$ up to $L_{\text {sys }}=128 \times 10^{5} \sigma$ for $\varepsilon=0.9999$. This system sizes were used for the simulation in Section 5.1. The systems look self-similar with this procedure, which indicates that the size of the clusters depends 


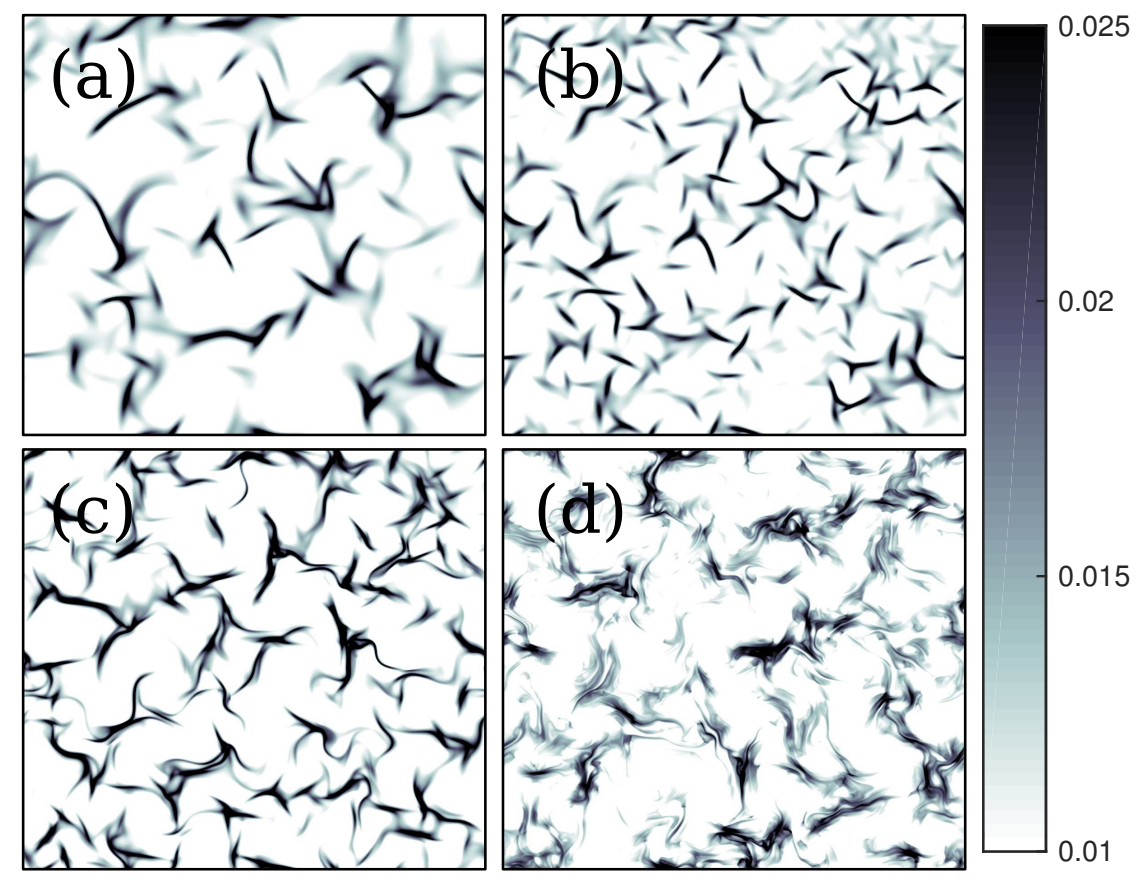

Figure 5.9: Freely cooling gas after transition to an ICS; $\varepsilon$ is constant and $\bar{\phi}=0.01$. The panels show snapshots for (a) $\varepsilon=0.5, L_{\text {sys }}=10240 \sigma$, (b) $\varepsilon=0.9, L_{\text {sys }}=25600 \sigma$, (c) $\varepsilon=0.99, L_{\text {sys }}=102400 \sigma$, (d) $\varepsilon=0.9999, L_{\text {sys }}=2048000 \sigma$

on $\varepsilon$, but the shape is in any case filamentary. Figure 5.9 shows pictures of the clusters in the ICS at different $\varepsilon$ and appropriate system-sizes.

We recognized that the formation of the clusters appears at a typical length scale. The typical cluster sizes depend on $\varepsilon$ and $\bar{\phi}$. This makes it necessary to rescale the system size if we change $\varepsilon$ or $\bar{\phi}$. In principle it is reasonable that the clusters appear at a typical length scale. We know already from linear stability analysis (see Section 2.9) that all wavevectors associated with the so-called "heat mode" $k_{H}$ which are smaller than $k_{H}^{*}$ are unstable and lead to a growth of these modes. On the other hand, the initial fluctuations $\Phi_{0}(k)$ have a distribution in $k$. Therefore it is reasonable that there is a preferred wavelength which will grow fastest. After formation the clusters at this wavelength can no longer be studied in terms of linear stability analysis because it is not valid anymore, therefore the ultimate fate of these density inhomogeneities cannot be determined from linear stability analysis.

In multiple works $[24,30,106,107]$ it is observed that the clusters grow till system size and it was shown that they can finally evolve into a sheared regime [106]. We want to note here that this phenomenon was observed in simulations which are close to the critical system size [106] or at really large filling fractions [107]. Results for dilute granular gases where the system size is much larger than the critical system size (which means about two orders of magnitude or even more) are still outstanding. The reason is that by doubling the system size, the density drops by a factor of four, which leads again to four times as large critical system size. This means that we need a very large number of particles, e.g. to run a MD simulation at $\bar{\phi}=0.01, \varepsilon=0.8$ and a size of $30 L_{\text {crit }}$ we would have to 
simulate about $10^{7}$ particles.

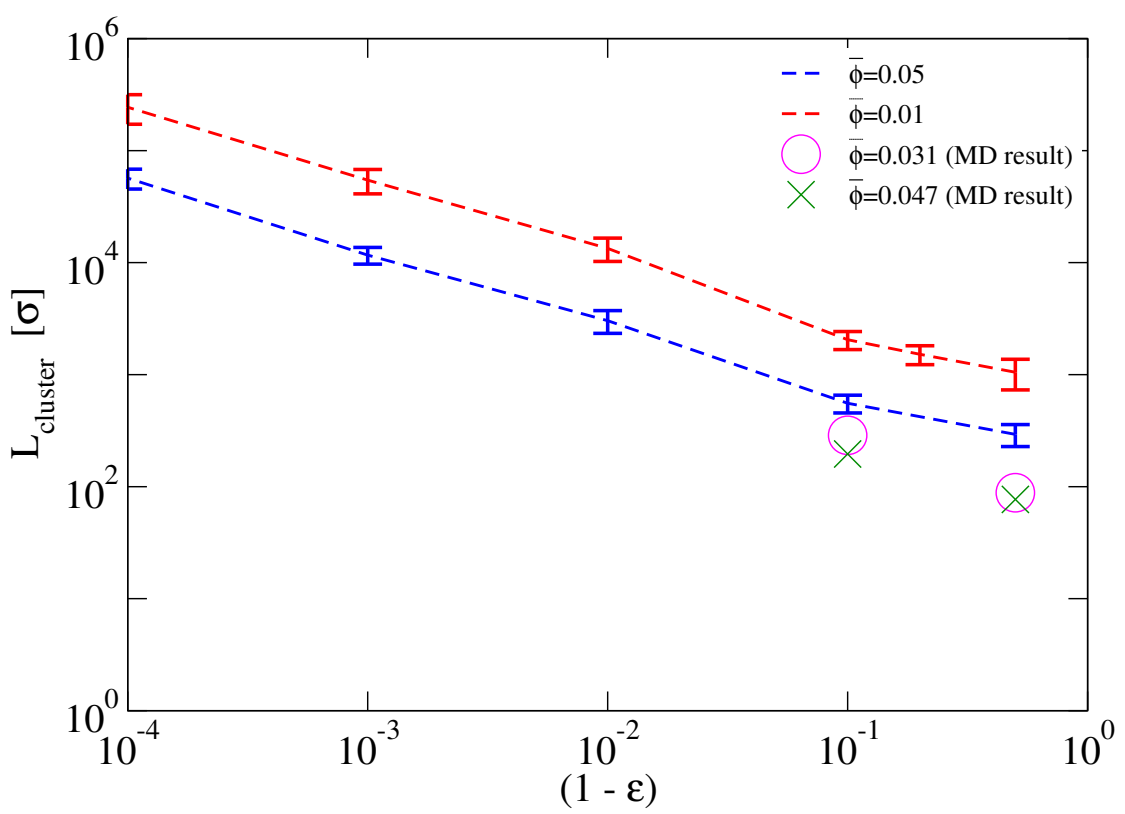

Figure 5.10: Characteristic cluster sizes in cooling granular gases after the transition to in ICS.
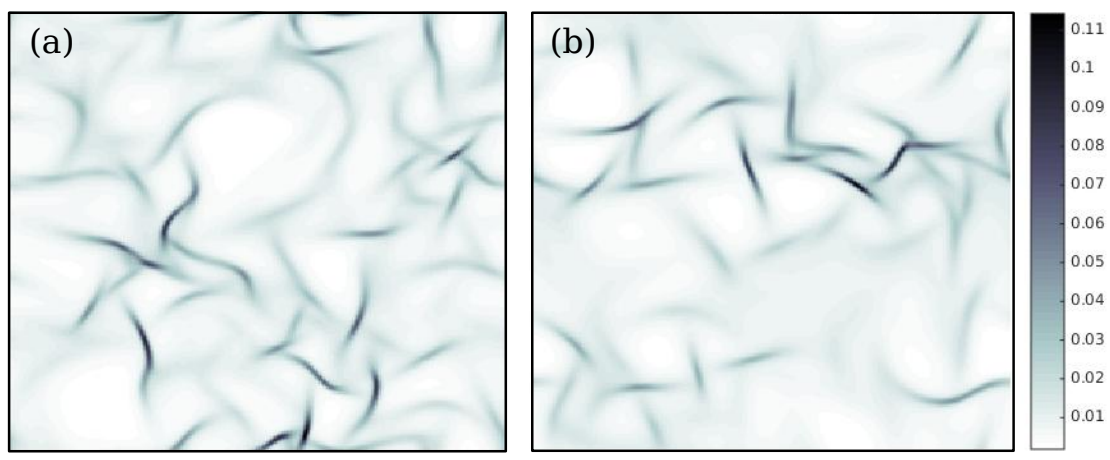

Figure 5.11: Cooling granular gas in the ICS with $\bar{\phi}=0.01, \varepsilon=0.9$. The initial convective velocities are of $\mathcal{O}\left(10^{-8}\right)$ in panel (a) and $\mathcal{O}\left(10^{-2}\right)$ in panel (b). There is no qualitative nor quantitative difference in the characteristic of the visible clusters.

Our simulations consistently find that when the clusters appear they have a characteristic length, which depends on $\varepsilon$ and $\bar{\phi}$. In Fig. 5.10 we plot the size of the clusters after the transition to the ICS. The linear size of the clusters is obtained by manual measuring of the length of 50 clusters in pictures like in Fig. 5.9. This gives us an indicator of how to set up our simulation to obtain good resolutions. We measured the length of clusters when they appear in MD, too (see MD results in Fig. 5.10). The results demonstrates the same qualitatively effects as obtained from DNS but the sizes are smaller by a factor of $3-5$. MD simulations are not possible at higher $\varepsilon$, because much more particles would have been necessary. In terms of the critical system size $L_{\text {crit }}$ (Eq. (2.89)), we find that 
the clusters have a characteristic size of about $2 L_{\text {crit }}$ (for small $\varepsilon$ ) to $8 L_{\text {crit }}$ (for $\varepsilon$ close to unity) for all data points in Fig. 5.10.

In Fig. 5.11 we see the clustering in systems with different initial convective velocities. We cannot observe any difference in the clustering behavior and conclude that a dependency on the initial convective fluxes is negligible. The results of this section show that there is a characteristic size of the clusters when they appear out of the HCS. I am very thankful to Samkit Shah for running the DNS and doing the related analysis in Fig. 5.11 and for $\bar{\phi}=0.05$ in Fig. 5.10. I also thank Artem Bolshakov for carrying out the MD simulations shown here. 


\section{Shock waves as the origin of clustering in a granular gas}

In this chapter we will study the influence of the local Mach number on freely cooling granular gases. A commonly used heuristic claims a correlation between the pressure and the temperature in the homogeneous cooling state, and claims an anticorrelation between the density and the pressure. We have evidence that this heuristic does not hold. It thus follows that a different mechanism must be the origin of the clustering in a cooling granular gas. Here we demonstrate that local subsonic convective fluxes are the seed for the clustering out of a homogeneous gas. The transition appears when convective fluxes turn supersonic. We perform DNS and MD simulations and bolster our arguments by analytical results. Our findings here give a new understanding on the onset of clustering and might be adapted to other fields of granular fluids and gases.

\subsection{Evolution of density, temperature and pressure fluctuations}

The behavior of granular shock-waves has already been studied by a number of authors [108-113]. One explanation for the appearance of granular shock-waves during the ICS $[108,110]$ was discussed in the framework of a sticky-gas-like behavior of granular gases in the ICS. Formally, the sticky gas is the limit for vanishing coefficient of restitution $\varepsilon \rightarrow 0$ of a granular gas. In this limit the pressure vanishes and the energy equation decouples from the momentum equation. In this case the NS equations simplify to the Burgers equation [2]. In [63] we have studied the dependency of the clustering on the 
global Mach number. We want to discuss now the following question: What is the origin of the clustering? A commonly used argument [2, 22, 23, 25, 30, 34, 37, 102] for the origin of clustering goes as follows. Small fluctuations of larger density lead to more collisions per unit time and thereby to a local drop in temperature. This energy loss leads in turn to a local drop of the pressure, which causes a positive feedback on the instability. Although the argument of the pressure instability as the origin of clustering is reasonable, we are not aware of any evidence in the literature coming from MD simulations nor from DNS. Those methods would provide a microscopic foundation to the claim of the origin of the clustering. This heuristic claim itself starts from a hydrodynamical point of view, so we set out to see a similar effect in our simulations of granular hydrodynamics. In this section we included the bulk viscosity $\eta_{B}$ from Eq.(2.59) in all simulations.

We start with a homogeneous state and study two different initial configurations: $(i)$ a small density offset in the center of the system

$$
\begin{aligned}
& \rho_{0}=\left\{\begin{array}{cc}
1.0 & , r \geq R \\
1.01 & , r<R
\end{array}\right. \\
& T_{0}=1.0 ;
\end{aligned}
$$

and (ii) a small temperature sink in the center of the system

$$
\begin{aligned}
& \rho_{0}=1.0 \\
& T_{0}=\left\{\begin{array}{cc}
1.0 & , r \geq R \\
0.99 & , r<R .
\end{array}\right.
\end{aligned}
$$

In both cases we observe a similar dynamics. We show these results in Fig. 6.1.

We observe an initial increase of the pressure leading to a first wave traveling away from the center, followed by a small drop in the pressure, which causes a second wave traveling towards the center. Then the pressure increases in the center above its average value again and does not drop below this average value anymore. This initial transient effect happens on a very small time scale, but still it sets the seed for the clustering which appears later. We want to stress the point that we are still in the HCS, which will stay homogeneous for orders of magnitude in time. This means that we observe that particles move towards a high pressure region. This is an unexpected phenomenon and seems to be unphysical, but we have to remember that our system is far away from equilibrium. We observe that the clustering becomes visible when the Mach number in the center reaches unity (see Fig. 6.1(c)).

It looks like the initially higher density in the center has caused the clustering in Fig. 6.1 because the clustering also appears in the center. A more careful look reveals that the clustering comes from the subsonic waves which are formed in the beginning because of the higher pressure region. We can control now the region of the clustering by changing the initial inhomogeneity in the center which affects the wave strength and thereby the location where the waves in the system turn supersonic. Figure 6.2 has a similar initial 

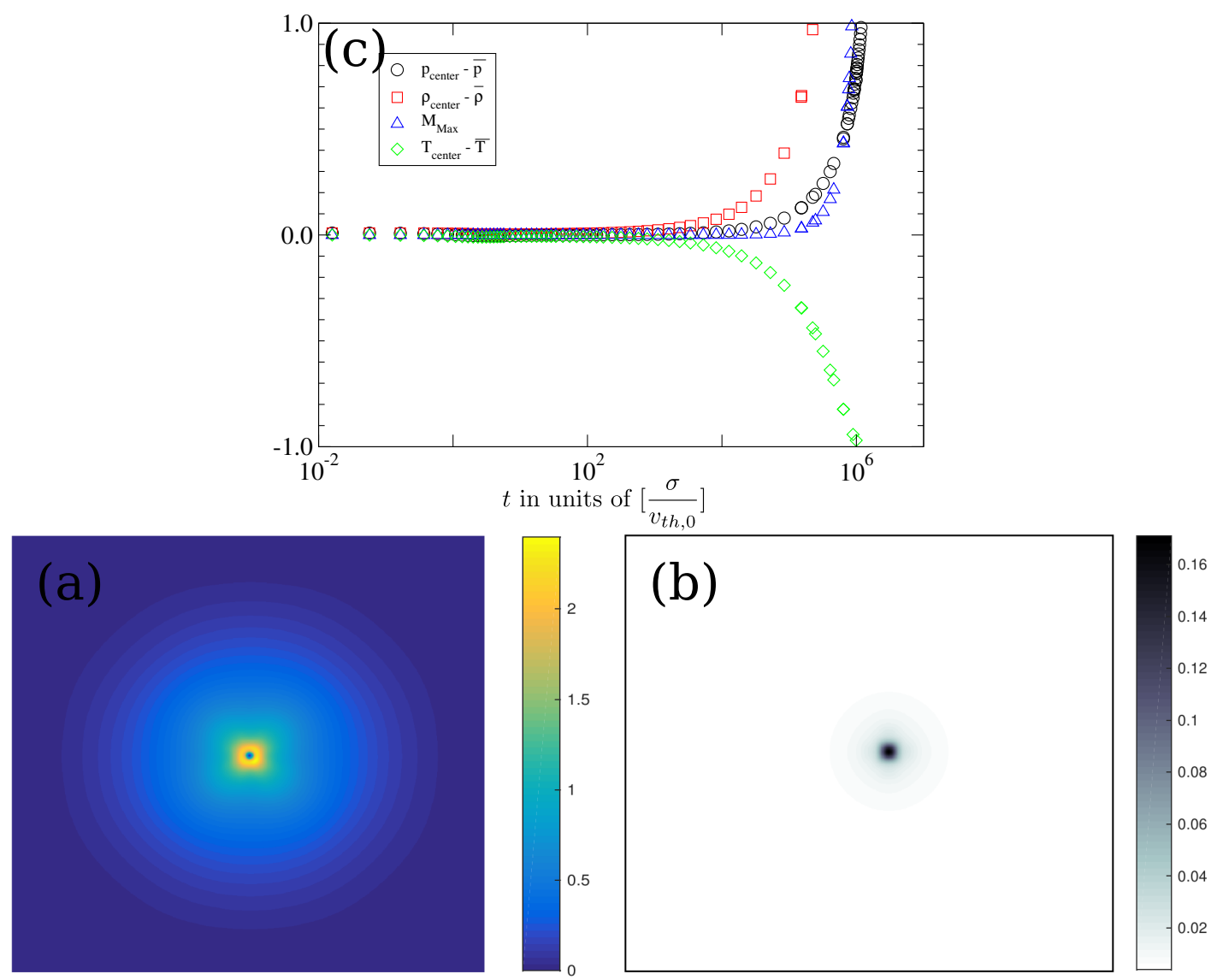

Figure 6.1: (a) Fieldmaps of the local Mach number and (b) local density at time $t \approx 10^{6}$. The system has periodic boundary conditions; the initial condition in Eq. (6.1) are used, the average filling fraction $\bar{\phi}=0.005$ and $\varepsilon=\varepsilon(T), \varepsilon(0)=0.9$. (c) Evolution of the pressure, density, temperature and the maximum Mach number in the center of 2D system. Apart from the local Mach number, the quantities are normalized and the deviation from the average value is plotted.
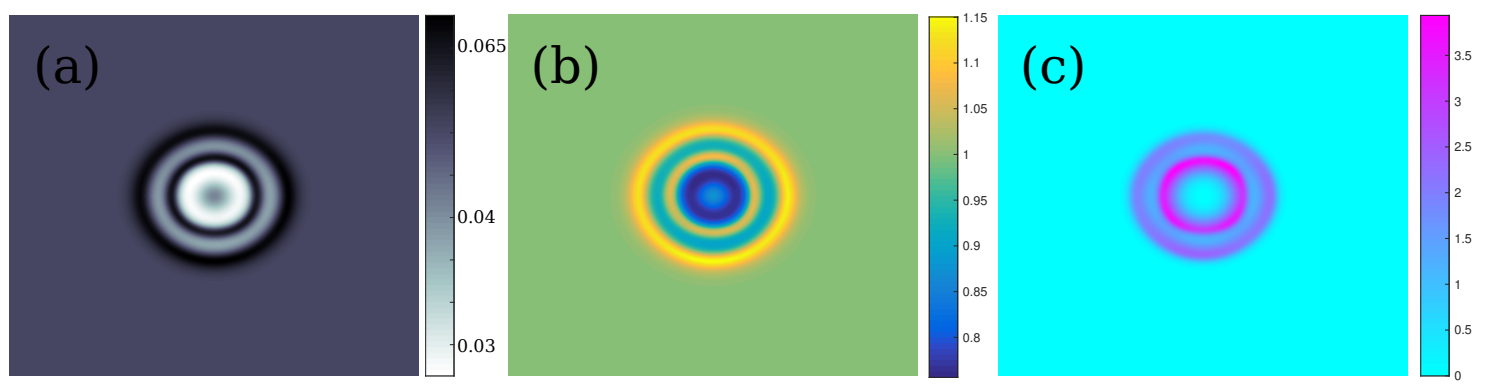

Figure 6.2: Final state of a freely cooling granular gas with. Fieldmaps of the local density (a), local pressure (b) and the local Mach number (c). The system has periodic boundary conditions; the initial condition are similar to Eq. (6.1), but the initial center density is $\rho=1.1, r<R$, the average filling fraction $\bar{\phi}=0.05$ and $\varepsilon=\varepsilon(T), \varepsilon(0)=0.9$. 
condition to Eq. (6.1) but the center density is set to 1.1 instead of 1.01. The center region in Fig. 6.2(a) becomes now a dilute region, which is against the heuristic and the region where the cluster forms is not in the center. In Fig. 6.2(c) we can observe that the clusters form along the surface of convective waves, which were initialized by the central inhomogeneity. Again we find from Fig. 6.2(b) that the clustering appears in regions of high pressure which indicates a positive correlation. We conclude from this that only the subsonic convective fluxes are relevant for the discussion about what causes the clustering. We do not observe any qualitative difference between the model of constant and variable coefficient of restitution (see also the attached movies centralFluctuation.avi and centralFluctuationViscoelastic.avi).

\subsection{The role of subsonic convective waves}

That the Mach number can reach high values is due to the fact that the kinetic energy associated with the propagation of the wave decreases in a much slower fashion than the temperature decay (Haff's law) ${ }^{1}$. Because of the different decay rates of convective and thermal flows the subsonic wave eventually becomes supersonic, this is when the clustering becomes visible (compare also the results in [63]). Figure 6.1 shows the evolution of this process. Once a wave become supersonic, the presence of a gas-solid transition at the shock front is known $[109,112,114]$. The related phenomenon in molecular gases is the compression shock, but a transition to a solid state is impossible in ordinary gases. The presence and the growth of supersonic waves in the ICS of a cooling granular gas are known. What we want to stress here is that supersonic waves do not appear in the ICS, but rather they are the reason for the ICS.

We want to support this statement by another simulation. If we observe a well defined subsonic wave in the system, we expect to see that with time this wave will turn to supersonic speed and a clustering will form at the wave front. Figure 6.3 shows the evolution of a system with a such a wave. Initially, this wave has a convective velocity six orders of magnitude smaller than the thermal velocity of the system. Thus, this is an extremely weak, subsonic wave. After some time, we find a transition to supersonic speed and the simultaneous onset of clustering. From this point on we also find the expected behavior for a compression shock wave in granular gases $[109,112,114]$. Here we stress again that the important point is that the initial negligible subsonic wave is the origin for the clustering inside the system.

The initial conditions we use for studying initial subsonic waves within a system of size

\footnotetext{
${ }^{1}$ Compare also Section 2.8 and our findings in Section 5.1.
} 


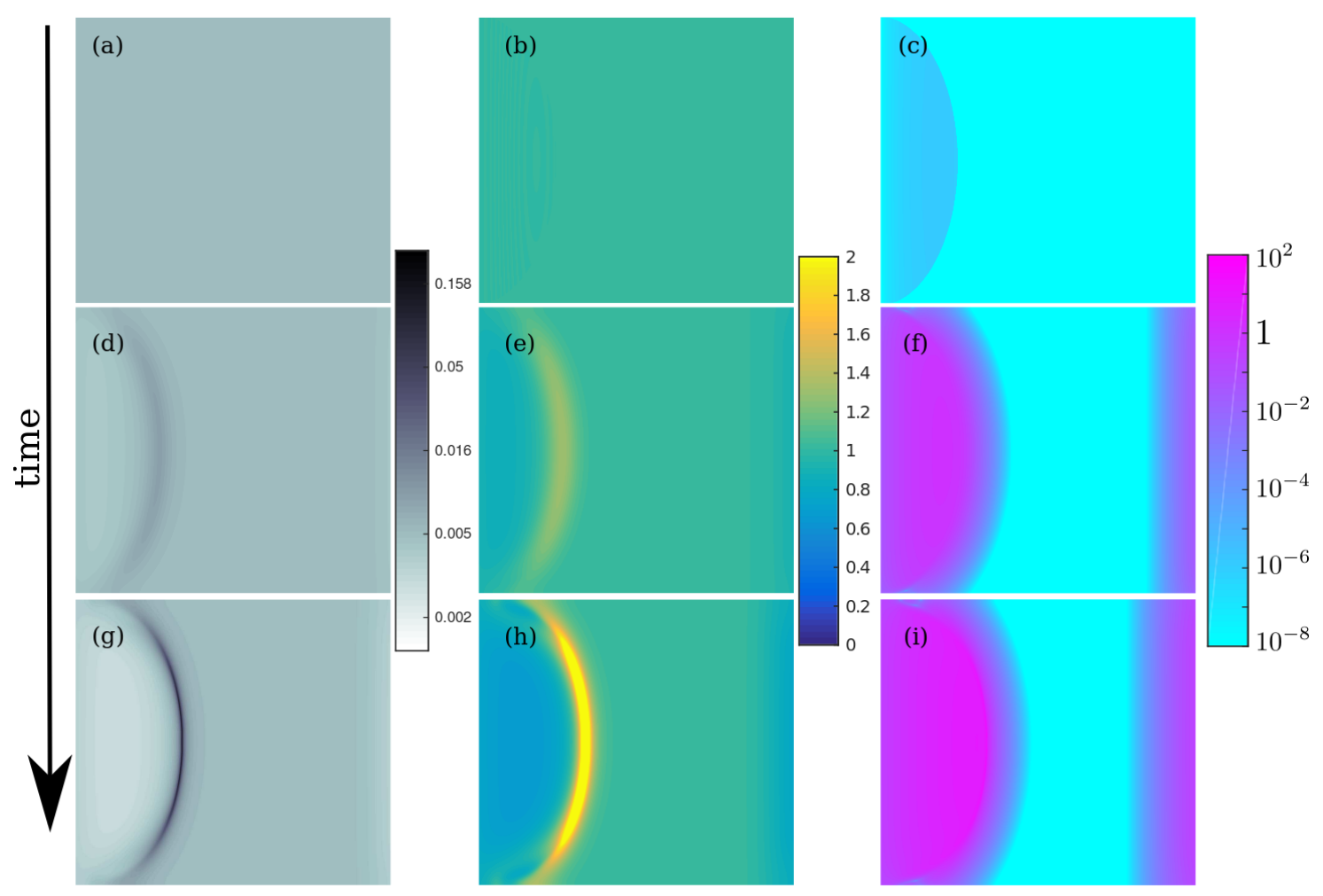

Figure 6.3: Evolution of a free cooling granular gas which was initialized with a subsonic convective wave. The initial subsonic wave has an amplitude of $10^{-6} v_{t h, 0}$. The panels (a),(d),(g) display the field maps of the density, panels (b),(e),(h) display the pressure in terms of the average pressure and the panels (c),(f),(i) display the local Mach number in the system. The subsonic wave in the initial state (panel (a)-(c)) turns to supersonic speed (panel (d)-(f)) and reaches eventually high Mach numbers (panel (g)-(i)). We can locate the transition from HCS to ICS at the time when the wave turns supersonic (panel (d)). The system has periodic boundary condition in $x$-direction and hard walls with no-slip boundary conditions in $y$-direction; the average filling fraction $\bar{\phi}=0.005$ and $\varepsilon=\varepsilon(T), \varepsilon(0)=0.9$.

$L_{x} \times L_{y}$ are

$$
\begin{gathered}
r=\sqrt{\left[1-\left(x-\frac{L_{x}}{4}\right)^{2}\right]+\left[1-\left(y-\frac{L_{y}}{2}\right)^{2}\right]} \\
v_{x}=\left\{\begin{array}{cc}
A r\left(1-\left(\frac{4}{L_{x}} x-1\right)^{2}\right) & , \quad x \leq \frac{L_{X}}{4} \quad \& \quad r \leq 1 \\
0 \quad & \text { else, },
\end{array}\right.
\end{gathered}
$$

where the initial amplitude $A=10^{-6}$. The convex initial condition were chosen to study a convex wave front as they typically appear within our DNS in a freely cooling granular gas. In addition we do not simulate a quasi one dimensional shock in this way but use a $2 \mathrm{D}$ surface. The quadratic profile along the $x$-axis in the velocity was chosen because of stability reason in our DNS. Different initial conditions show the same qualitative behavior, so that we can conclude that the shape of the initial wave is not important for 
our following argumentation. In the beginning, see Fig. 6.3(a-c), a convective velocity $10^{6}$ times smaller than the thermal velocity is injected into the system. Over time the temperature decreases, but convective velocities stay nearly constant, thereby the Mach number of the wave increases in time. Figure 6.3(d-f) display the density, pressure and local Mach number, respectively, at the time when the wave propagation turns supersonic, which marks the onset of the clustering. We can see from panel (f) that the Mach number reaches unity at this time. The pressure in the dense region grows and eventually we find clustering characterized by high pressure, which makes sense in the context of a shocks wave. The Mach number keeps increasing and we observe a compression shock wave which drives the clustering to even higher densities. The steady increase of density in a plane shock wave was also observed recently in [113], here we have shown in addition that eventually every wave turns into a shock wave.
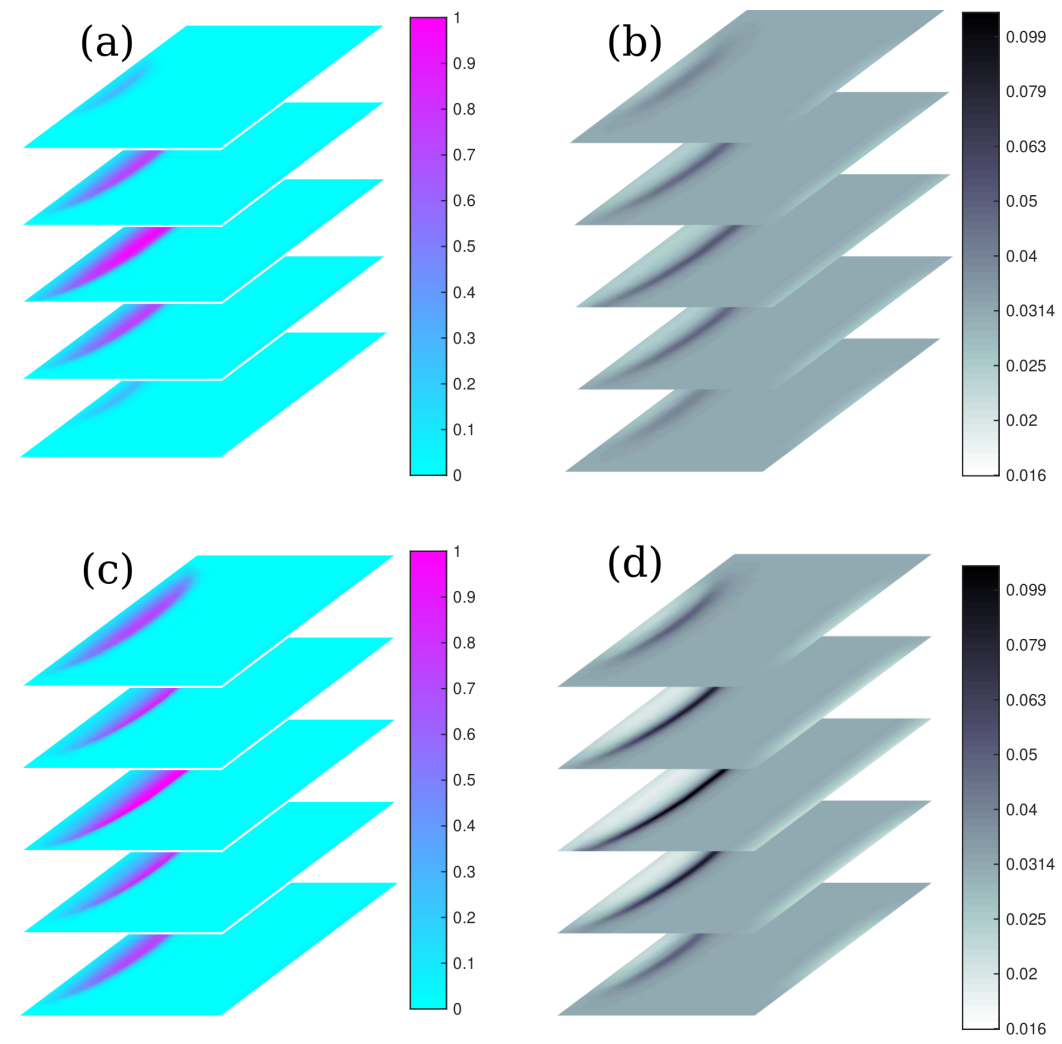

Figure 6.4: Evolution of a freely cooling granular gas which was initialized with a subsonic convective wave in $3 \mathrm{D}$. The initial subsonic wave has an amplitude of $10^{-4} v_{t h, 0}$. The panels (a),(c) display cross sections of the fieldmap of the local Mach number and panels (b),(d) display cross sections of the field map of the density in the system. The subsonic wave in the initial state turns to supersonic speed (panel (a)) and reaches eventually high Mach numbers (panel (c)). We can locate the transition from HCS to ICS at the time when the wave turns supersonic (panel (b)). The system has periodic boundary condition in all directions; the average filling fraction $\bar{\phi}=0.01$ and $\varepsilon=\varepsilon(T), \quad \varepsilon(0)=0.9$. The system is not as stable as the $2 \mathrm{D}$ simulation in Fig. 6.3, therefore the final state(panel (c),(d)) is not as far developed in time as it is in Fig. 6.3(g)-(i).

A study of the 3D case reveals the same qualitative behavior. Figure 6.4 shows the 
evolution of cross sections of a 3D cooling granular gas which was injected with an initial subsonic wave. Again we can observe that the clustering starts at the threshold from subto supersonic flow (see panels (d)-(f) in Fig. 6.4).
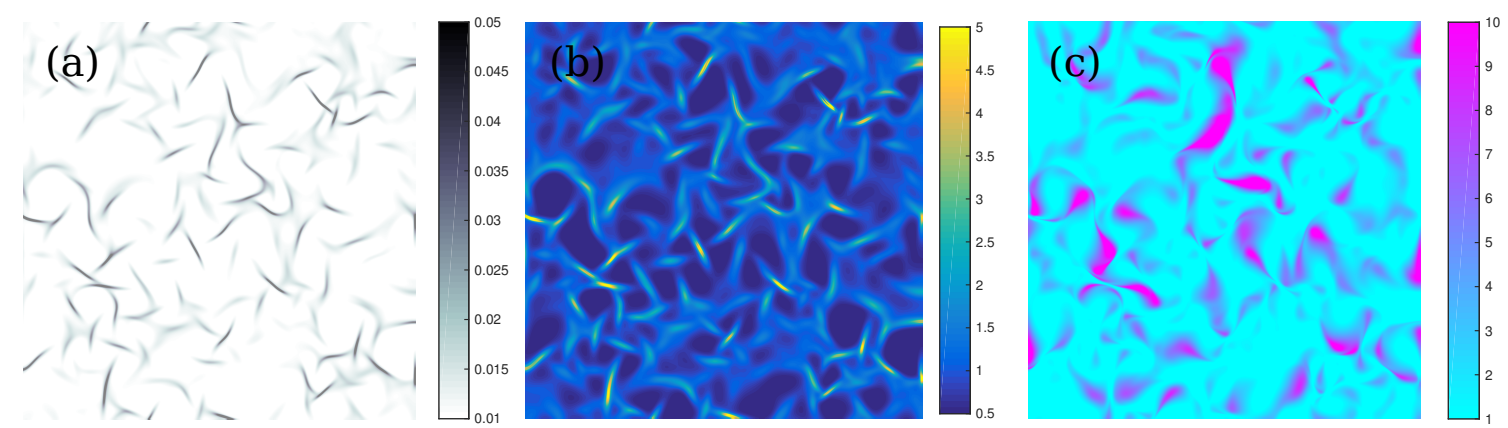

Figure 6.5: A granular gas in the ICS. (a) field map of the filling fraction. (b) pressure in the system. The high correlation between pressure and density is obvious. (c) field map of the local Mach number. The cutoff is set at $\mathcal{M}=1$ to distinguish between suband supersonic waves. The system has periodic boundary conditions, the average filling fraction $\bar{\phi}=0.01$ and $\varepsilon_{\text {const }}=0.9$.

We can now generalize our finding in this Chapter to any freely cooling granular gas. Figure 6.5 shows the local filling fraction and Mach number of an unperturbed, freely cooling granular gas in the ICS. The figures make clear that the clusters are characterized by high pressures compared to the average pressure in the system and it also shows that the clusters form on the leading front of the supersonic regimes.

\subsection{Comparison with molecular dynamical simulations}

To gain more confidence in our results we run MD simulations and compare the results with our DNS. As we did in the last section for DNS, also in the MD system we inject a convective wave which is subsonic in the homogeneous cooling state. Figure 6.6 displays the results of MD which are indeed similar to the DNS in Fig. 6.3. The subsonic wave is turning supersonic and the clustering starts at the surface of the wave. The MD simulations are in complete agreement with the finding of the DNS. The effect becomes more visible if we use more particles inside the system (see also the attached movie CurvedSeededShock.avi).

In Fig. 6.7 we varied the strength of the initial convective wave and compared the results of MD simulations with DNS. The initial shape of the wave is define in Eq. (6.3) (see Fig. 6.3 and Fig. 6.7), but we vary the amplitude. We can clearly observe that the onset of clustering in the cooling granular gas does strongly depend on the strength of the initial convective flux. In order to measure the clustering in Fig. 6.7 within the MD simulations, we employed the following technique. The system of $N=40.000$ (640.000) particles was divided into 625 (10000) boxes. If a finite volume contains more than $20 \%$ of the average density, we count the box as a dense box. The time at which more than 25 (100) dense 

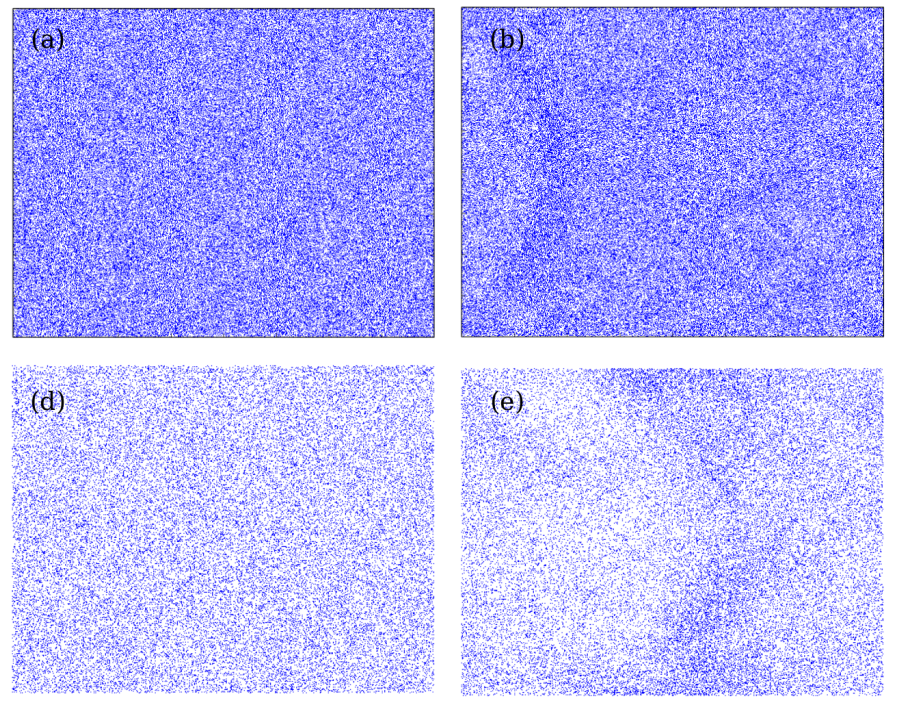

time

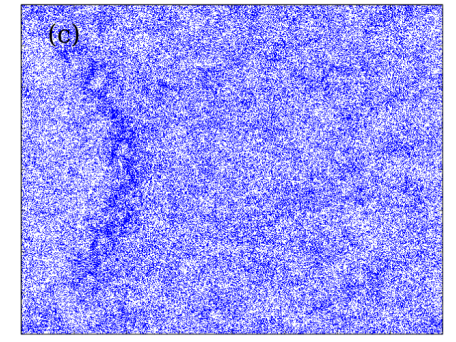

(f)

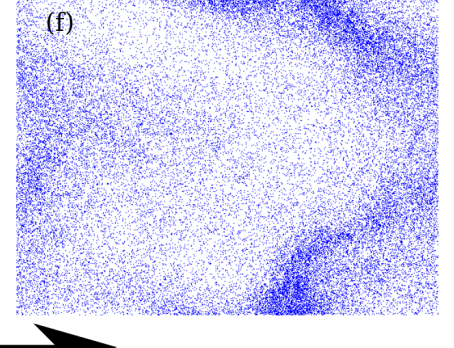

Figure 6.6: MD simulations of two freely cooling systems. (a), (b), (c) show the evolution of a system with $L_{\text {sys }}=8000 \sigma, 2.560 .000$ particles and $\varepsilon=0.9$. (d), (e), (f) show the evolution of a system with $L_{\text {sys }}=1000 \sigma, 40.000$ particles and $\varepsilon=0.9$. Both simulations start from a homogeneous state with a well defined initial convective flow as in the DNS in Fig. 6.3. The initial convective flux is $10 \%$ of the thermal velocity in the top panels and $45 \%$ in the bottom ones. The critical system size for both configurations is about $360 \sigma$.

boxes appear defines the onset time of clustering in our MD simulation. Inside the DNS we measure the onset of clustering as the time when the local density inside a finite value exhibits $50 \%$ larger of the initial value. That the threshold for the onset of clustering is slightly different has small impact on our results because the clustering appears very fast compared to the logarithmic time scale intrinsic to the data plotted in Fig. 6.7.

We find that as the strength of the injected subsonic wave grows the clustering time decreases. For large initial subsonic wave strengths we find a very good agreement between the DNS and MD results. The results of the MD simulations reach a plateau if the initial flux is below a certain value. This "natural" onset time of clustering is because the MD simulation cannot be made more homogeneous within our simulation setup. A study of systems which contain more particles but at constant density might give results even closer to the DNS. Nevertheless, we find a good agreement between the results of MD simulations and DNS for large initial convective velocities and we can conclude that the MD simulations support our hydrodynamical results and give stronger confidence in our findings. A further study of a system with a much larger number of particles could reveal the natural strength of initial convective fluxes because of fluctuations within local velocities. From this it is possible to derive the "characteristic" cluster time of such a homogenouse granular gas.

In this section we have demonstrated that the clustering is generated by small convective fluxes in the HCS, not by density or pressure fluctuation (which may also cause convective fluxes). Once the waves are formed, these small convective flows are quite stable in time 


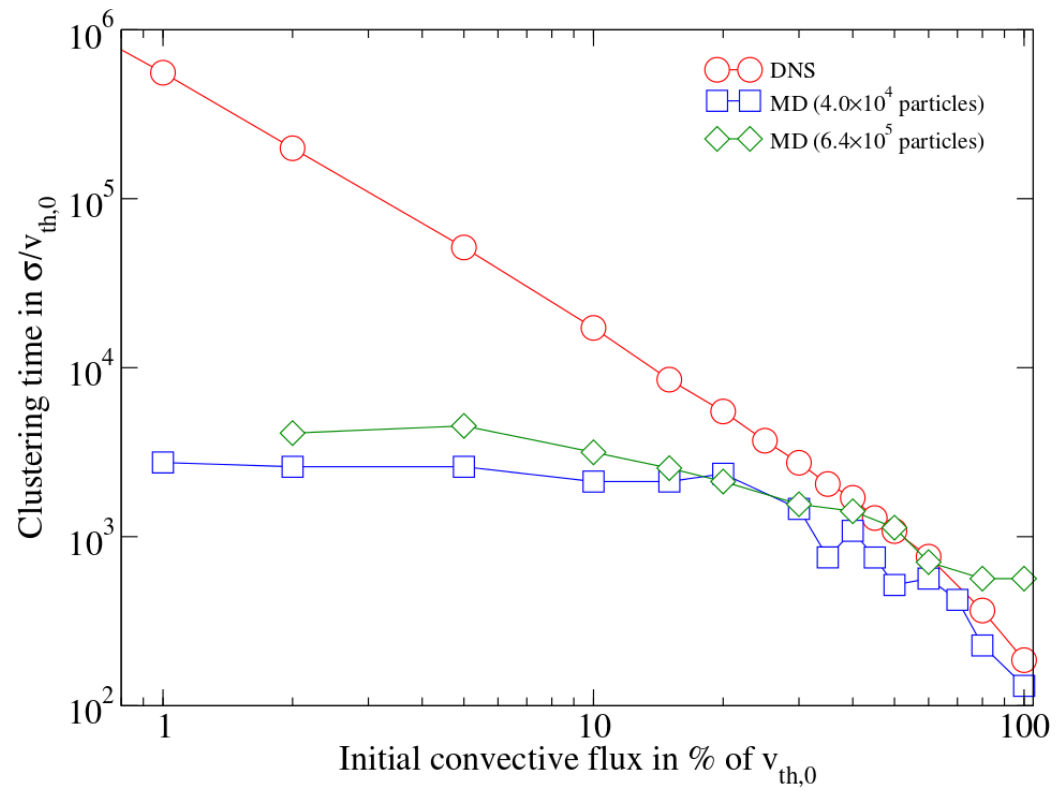

Figure 6.7: Dependency of the onset time of clustering on the initial strength of a convective wave. The results are taken from MD simulation with $N=40.000, N=640.000$ particles and from DNS. In all simulation the filling fraction is $\bar{\phi}=3.14 \%$ and $\varepsilon_{\text {const }}=0.9$. Results for MD simulation are obtained from an average over $2-5$ simulations with different initial random particle locations and velocities. The lines are a guide to the eye.

and decay in a much weaker fashion than the temperature, thereby they eventually become supersonic and from this moment on they act like a compression shock-wave inside the granular gas. In addition we find from our simulations that the pressure increases inside dense regions (compared to the average pressure) and does not vanish (this agrees with the results in [113]), therefore we cannot observe a sticky gas limit as proposed in [108, 110], which means that the Burgers equation is not a valid representation of the ICS. The results in this section are in complete agreement with our findings in [63]. In addition these results explain why the anticorrelation between temperature and density vanishes, which we described in Section 5.3. The heuristic argument that the temperature drops when the density raises due to more collisions between the particles is not valid in the presence of shock-waves. On the front of a shock wave both the temperature and the density rise, which is also true for ordinary and granular gases [113]. Finally, we can summarize that the heuristic argument, that low pressure regions are the origin for clustering, could not be validated neither with our hydrodynamic observations nor with our MD simulations.

We are very thankful for the collaboration with Artem Bolshakov, who has carried out the MD simulations and provided us with the related data. 


\subsection{Correlation between density, pressure and temperature in the homogeneous cooling state}

As a simple measure for the characterization of the system we consider the calculation of Pearson's correlation coefficient

$$
\operatorname{Cor}(f, g) \equiv \frac{\langle(f-\langle f\rangle)(g-\langle g\rangle)\rangle}{\sigma_{f} \sigma_{g}},
$$

where $\sigma_{f}, \sigma_{g}$ are the standard deviations of $f$ and $g$, respectively. To disentangle physical effects from subtle artifacts which are inherent to any concrete calculation we carried out both DNS and MD simulations. While DNS, with its field description, gives access to large hydrodynamic structures, it may miss subtle correlations rooted in the discrete nature of grains. Hence, MD simulations are advisable in this context. However, the limited size of an MD system, coupled with the complexity of extracting appropriate statistical averages calls for a dialectic comparison between the two models.
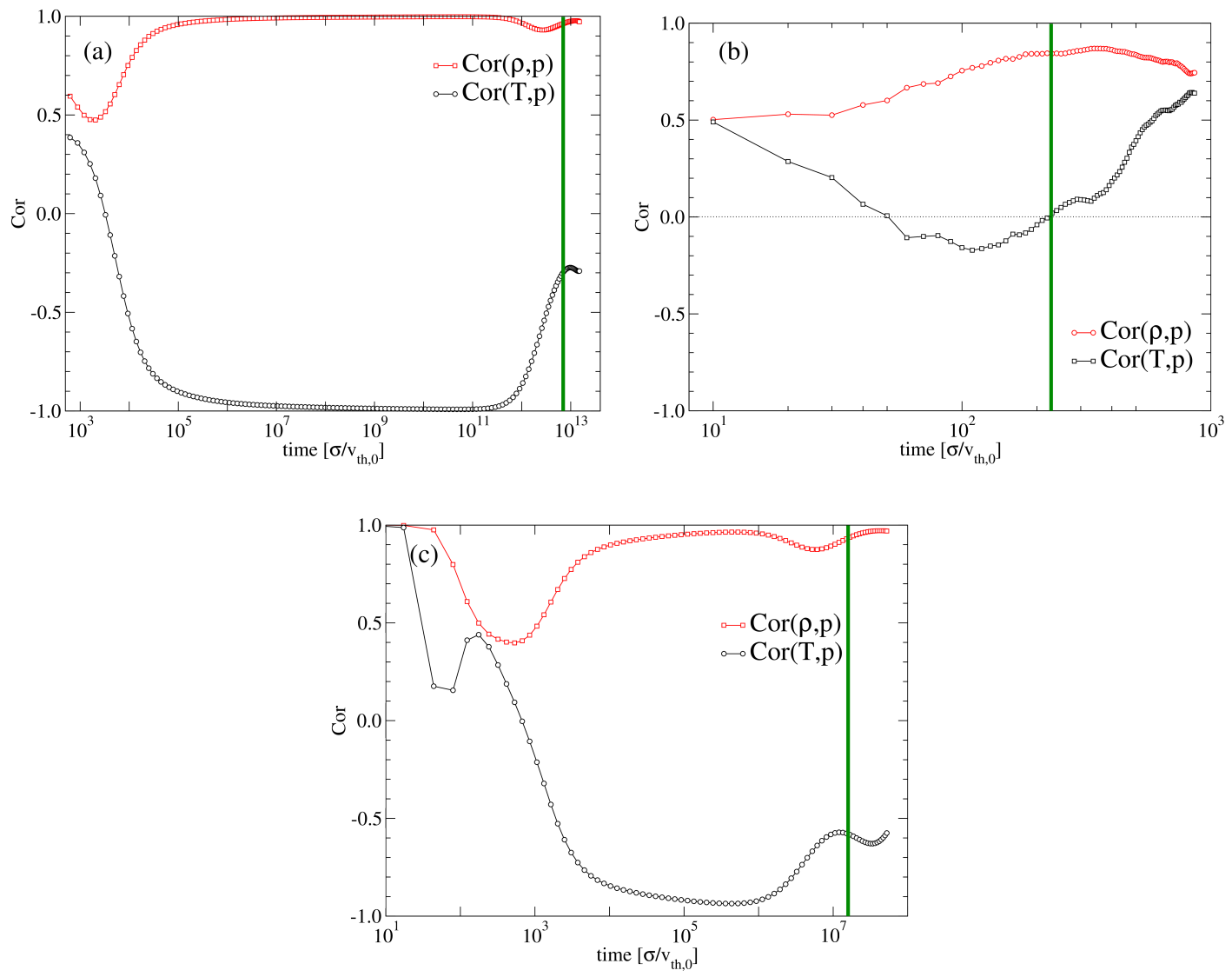

Figure 6.8: Correlation between density and pressure for free cooling granular gas. (a) displays some DNS calculations as in Fig. 6.5 (2D system). (b) displays data from MD simulation with $\varepsilon=0.7$ and 160.000 particles (2D system). (c) displays DNS results of a free cooling 3D system with $\varepsilon=0.9$ and $\bar{\phi}=3.14 \%$. The green line marks the time around which the transition to ICS appears. 
In Fig. 6.8 the correlations $\operatorname{Cor}(\rho, p)$ and $\operatorname{Cor}(T, P)$ are shown. During the entire HCS the DNS results (panel (a)) exhibit a correlation close to unity between $\rho$ and $p$ and an anti-correlation between $T$ and $p$. In the ICS the anti-correlation seems to fade away. The MD simulations (panel (b)) display a positive correlation $\operatorname{Cor}(\rho, p)$, and the correlation between $T$ and $p$ drops in the HCS. The correlation $\operatorname{Cor}(T, p)$ raises again when the system turns to the ICS. Both DNS and the MD simulation are initialized with homogeneous distributions of $T, \rho$ or particle positions and velocities; the values are randomly assigned. In the DNS and in the MD simulation we run simulation with and without an initial equilibrium time of $t_{e q}=10^{4} \frac{\sigma}{v_{t h, 0}}$; we cannot find any qualitative difference in the results. In the MD simulations the initial equilibration enlarges the duration of the HCS.

For the initial equilibration the $\varepsilon=1$ limit was chosen and the time was reset after the initialization process. The initial transient effects in the first $t=10^{4} \frac{\sigma}{v_{t h, 0}}$ appears with and without initial equilibration, it might be an artifact of self-correlation in both cases. In the DNS we can clearly observe this transient effect and after two orders of magnitude in time the transient effects vanish. The MD simulation starts much earlier to cluster and it looks as the transient time to reach the final value of correlation in the HCS could not even be reached. Nevertheless, we can still find the same trend in both simulation and we can definitive conclude that there is a positive correlation $\operatorname{Cor}(\rho, p)>0$ and no positive correlation between $T$ and $p$. Because we have conducted simulations in both $2 \mathrm{D}$ and 3D, and found no qualitative difference (see Fig. 6.8(c)) we can also conclude that the results are independent of the dimensionality.

As far as we can observe in the simulations, even the system in the ICS exhibits a positive correlation between $\rho$ and $p$. In the HCS we observe a nearly total anti-correlation between density and temperature (see Fig. 5.7) as it is expected from the heuristic argument for the origin of the clustering. What is not expected is a positive correlation between the pressure and the density; from the heuristic we would expect to see a drop in the pressure in regions with higher densities. We can conclude that we find from our DNS and MD simulations evidence that there is a positive correlation between the pressure and the density for the entire HCS and even in the ICS.

\subsection{Correlation between density, pressure and temperature in the linear stability analysis}

To support our arguments of this chapter we will show here that the linear stability analysis of the homogeneous cooling state predicts our claims. The task is to express to evolution of perturbations in the linearized system and then to figure out what is the relative phase between the fluctuations. We use the same expressions of perturbations in Fourier space $\delta, \omega, \theta$ as in Section 2.9. The evolution can be written in terms of the eigenvectors and 
eigenvalues of $\underline{\mathbf{M}}$ (see Eqs. (2.85) and (2.87)) as

$$
\left(\begin{array}{c}
\delta(\alpha) \\
\omega(\alpha) \\
\theta(\alpha)
\end{array}\right)=\sum_{i=1}^{3} C_{i} \boldsymbol{\Phi}_{i} \mathrm{e}^{\lambda_{i} \alpha}
$$

where the transformed time $\alpha$ is given by Eq. (2.82). We know already from Section 2.9 that for any $k<k_{c}$, in the long term only the heat mode gives contribution to the solution (compare Fig. 2.4). Therefore we assume without loss of generality that $\Phi_{1} \mathrm{e}^{\lambda_{1} \alpha}$ is associated with the heat mode. In addition we point out that $\lambda_{1}$ is real, which comes from the fact that there are three eigenvalues and if $\lambda$ would be complex, the complex conjugate would also appear as an eigenvalue. We express the pressure in the dilute limit up to linear order as

$$
p=p_{0}\left(1+\pi_{p}\right)=\rho_{0} T_{H}(1+\delta+\theta),
$$

where $\pi_{p}=\delta+\theta$ is the pressure perturbation. We want to know the correlation between $\delta$, $\theta$ and $\pi_{p}$. We introduce the notation $\boldsymbol{\Phi}_{i}=\left(\begin{array}{l}\phi_{i 1} \\ \phi_{i 2} \\ \phi_{i 3}\end{array}\right)$ and obtain the normalized perturbations in the limit $\alpha \rightarrow \infty$

$$
\begin{aligned}
\lim _{\alpha \rightarrow \infty} \delta & =\lim _{\alpha \rightarrow \infty} \frac{\sum_{i=1}^{3} C_{i} \phi_{i 1} \mathrm{e}^{\lambda_{i} \alpha}}{\left|\sum_{i=1}^{3} C_{i} \phi_{i 1} \mathrm{e}^{\lambda_{i} \alpha}\right|}, \\
& =\frac{C_{1}}{\left|C_{1}\right|} \frac{\phi_{11}}{\left|\phi_{11}\right|} \\
& =\mathrm{e}^{\mathrm{i} \gamma} \frac{\phi_{11}}{\left|\phi_{11}\right|} \\
\lim _{\alpha \rightarrow \infty} \theta & =\mathrm{e}^{\mathrm{i} \gamma} \frac{\phi_{13}}{\left|\phi_{13}\right|} \\
\lim _{\alpha \rightarrow \infty} \pi & =\mathrm{e}^{\mathrm{i} \gamma} \frac{\phi_{11}+\phi_{13}}{\left|\phi_{11}+\phi_{13}\right|}
\end{aligned}
$$

where $\gamma=\gamma\left(\delta_{0}, \theta_{0}\right)$ is a phase shift which is related to initial perturbations $\rho_{0}, \theta_{0}$. Because the phase shift $\mathrm{e}^{\mathrm{i} \gamma}$ is a common factor to every component, we can conclude that the initial perturbations are not relevant for the final correlation of $\delta, \theta$ and $\pi_{p}$.

Figure 6.9 shows the relation in the complex space between $\delta, \theta$ and $\pi_{p}$ for two different initial conditions. We can observe that the density fluctuations are perfectly in phase with the pressure fluctuations and both are anti-correlated to the temperature fluctuations. Therefore we can conclude that the heuristic argument cannot even be reproduced by the linear stability analysis.

Finally, we can state that we haven't found any hint to confirm the heuristic argument 

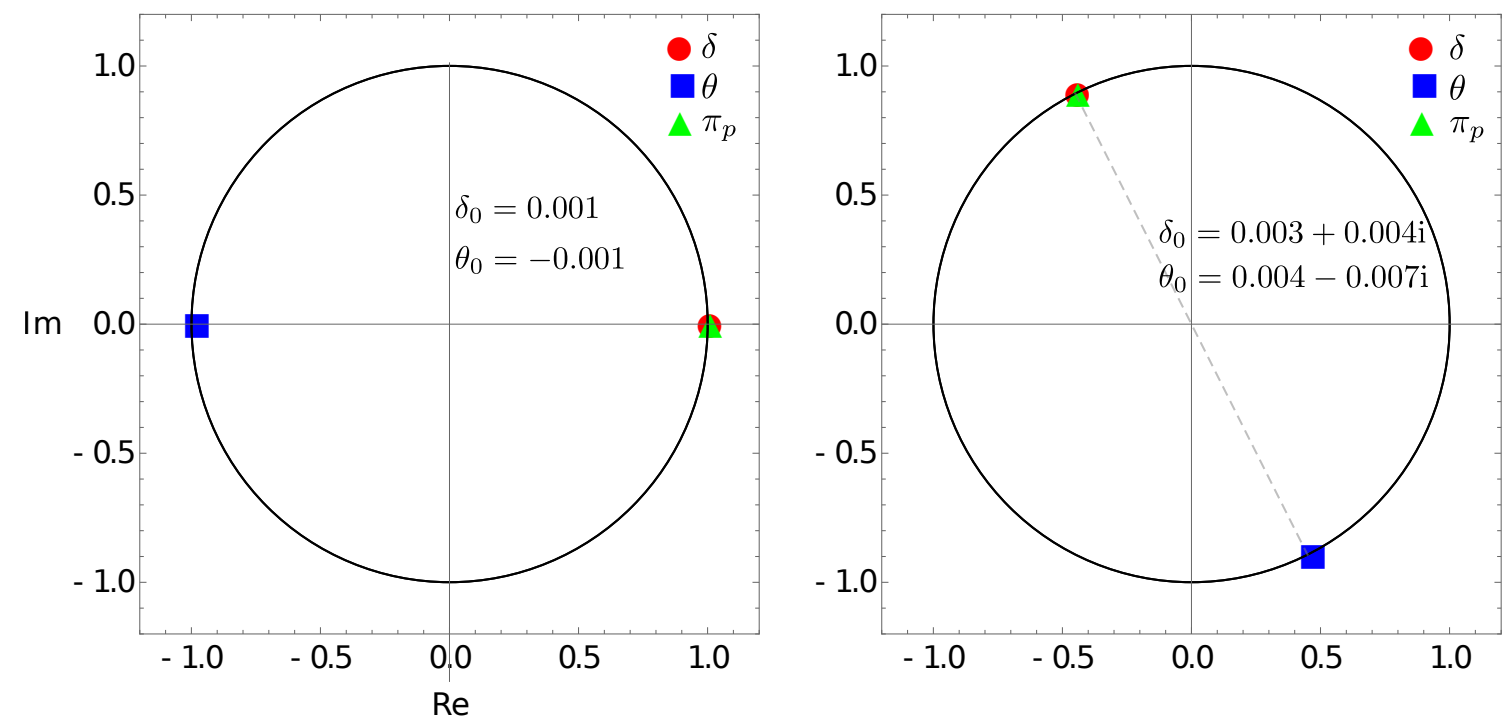

Figure 6.9: Asymptotic normalized perturbations for two different initial perturbations. A correlation between $\delta$ and $\pi_{p}$ can be observed. The temperature perturbation is anticorrelated to $\delta$ and $\pi_{p}$.

that the pressure drops in denser regions. The DNS, the MD simulations and the linear stability analysis indicate the opposite behavior, namely that particles move into a high pressure region and the cluster form in regions of high pressure compared to the average pressure in the system. We are thankful for the collaboration with Dr. Vinay Gupta, who collaborated with us on the linear stability analysis.

We have shown in this chapter that the heuristic argument, a local density fluctuation followed by a local pressure drop, cannot be the reason for the onset of clustering. This heuristic argument was never disproved and found its way in the textbooks on granular matter. We showed that it is time to revisit this point. In fact we proved that the existence of small local convective fluxes are the reason for clustering. We have shown that these convective fluxes turn eventually into shock waves, which mark the transition from HCS into ICS, and drive the clustering. That means, in addition, that the ICS can be described by granular shock waves. Further research and a deeper understanding of this granular shock waves will provide additional information about the behavior of a granular system in the ICS. 


\section{Declustering in a granular gas as a finite size effect}

The existence of dense clusters has been shown to be a transient phenomenon for realistic models of granular collisions, where the coefficient of restitution depends on the impact velocity. In this chapter we demonstrate that upon cluster formation the granular temperature and the convective kinetic energy couple and both follow Haff's law. Furthermore, we show that clusters will eventually dissolve in all finite-size systems. We find the strong power law $t^{\prime} \propto L^{12}$ for the dependency of the declustering time on system size. Our results imply that only in systems close to the initial critical system size both the clustering and declustering transitions are observable.

Here we show that the disappearance of clusters in granular gases is in principle a finite-size effect. As the granular temperature decreases the critical system size increases until it reaches the size of the system, upon which the density inhomogeneities dissolve and the system returns homogeneous. However, the steep dependence of the time of homogenization on system size implies that for any amount of dissipation a system size can be found where the gas will remain heterogeneous at any realistic time scale.

Unless otherwise stated all results in this chapter are based on perform simulations in 2 D with periodic boundary conditions on a $128 \times 128$ grid. We measure the linear system size $L_{\text {sys }}$ in units of $\sigma$. For reasons of self-consistency we then use the transport coefficients in Eq. (2.60), where $\varepsilon=\varepsilon(T(\vec{r}, t))$. We discussed the validity of the model in Section 2.7. We have compared our results with the full viscoelastic model in Eq. (2.61) and do not observe any qualitative nor significantly quantitative difference. In this chapter we do not include the bulk viscosity $\eta_{B}$ in Eq. 2.56. 


\subsection{Long time behavior of granular gases}

We are interested in the clustering dynamics during the ICS. In the ICS dense clusters form which are characterized by huge gradients in the hydrodynamic fields and eventually lead to a density collapse. It might be questionable if the NS approach is still valid in such extreme situations. It is known from linear stability analysis, that clustering appears if the system is larger than a critical value $L_{\text {crit }}$ (see Section 2.9). We found that the clustering close to the critical point is less strong, which means we can avoid the density collapse and are able to study the ICS (see Section 7.2 for more details). Heuristically this is reasonable if there is an $\varepsilon$ and $\bar{\phi}$ dependent characteristic size of the clusters when they form. In the case of a small system then the clusters would form at their characteristic size but are not able to accumulate enough particles to reach maximum packing fraction. Nevertheless, more studies especially from MD simulation could be of future interest here.
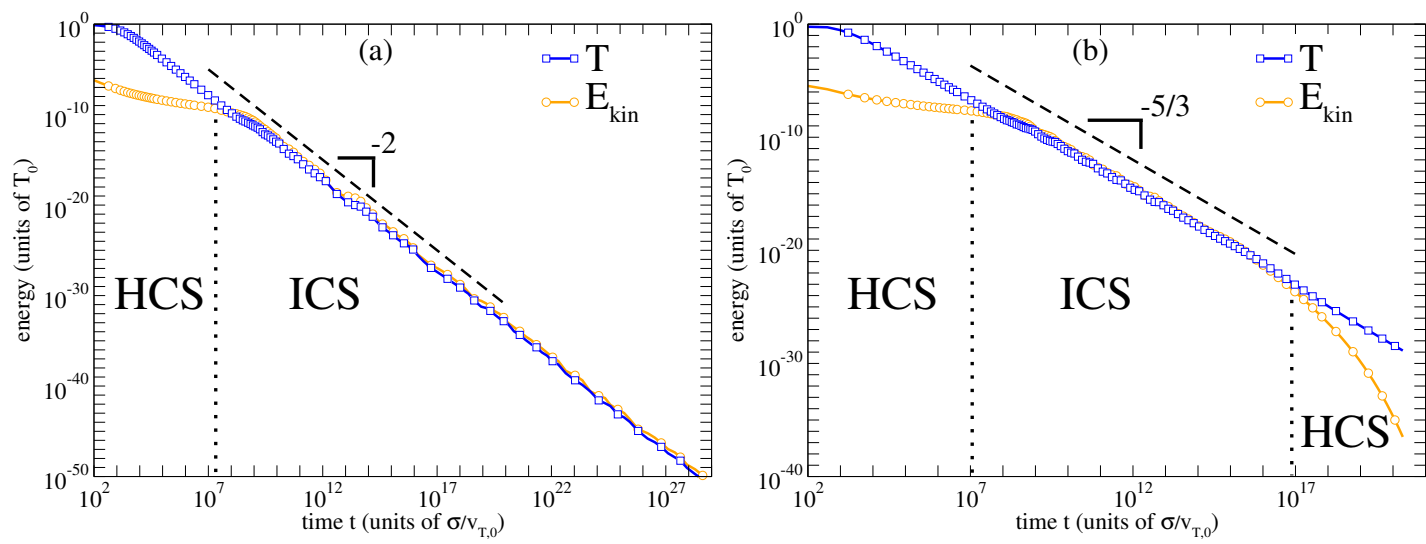

Figure 7.1: Temporal evolution of the average kinetic energy $E_{k i n}$ and the average granular temperature $T$ for a granular gas with average filling fraction $\bar{\phi}=0.05$. (a) Granular system with fixed coefficient of restitution $\varepsilon=0.99$ and $L=1920$. (b) Granular system with variable coefficient of restitution and initial value $\varepsilon_{0}=\varepsilon(t=0)=0.99$ and $L=5120$. The clustering appears at the crossover of thermal energy and kinetic energy of convective velocities. In both cases thermal and kinetic energy couple at the transition point and undergo a Haff's cooling afterwards. In the case of viscoelastic particles, we can observe a decoupling of thermal and kinetic energy, which sets the onset point of declustering.

It is useful for our discussion below to also consider the temporal evolution of the average kinetic energy of the system $E_{k i n} \equiv \sum_{i} \rho_{i}{\overrightarrow{v_{i}}}^{2} / \sum_{i} \rho_{i}$ and the average temperature $T \equiv \sum_{i} \rho_{i} T_{i} / \sum_{i} \rho_{i}$, where $i$ is the index of the finite volume in which the system is divided. Figure 7.1(a) shows the evolution of $E_{k i n}$ and $T$ for a gas with $\varepsilon=$ const, and panel (b) the same quantities for a gas of viscoelastic particles $(\varepsilon=\varepsilon(T)$ ). Haff's law (see Section 2.8) with the appropriate $\alpha$ is recovered for both models. We observe two temporal regimes when $\varepsilon=$ const: a HCS when $T$ follows Haff's law, and $E_{k i n}$ follows a power law with a very small exponent; an ICS when the two curves meet and $E_{k i n}$ displays a dynamical transition to a Haff-like dependence. The transition from HCS to ICS occurs when convective and 
thermal velocities are of the same order of magnitude [63]. The Mach number has been shown to capture the dynamical evolution of the HCS [63] (see Chapter 5). These two quantities remain coupled for the entire remaining evolution of the gas. The beginning of the second regime marks also the transition from the HCS to the ICS with the clustering of the gas. For the model of viscoelastic particles, three temporal regimes can be recognized. Similarly to the previous case the gas starts from a HCS, transitions into a ICS which is also characterized by the coupling of $E_{k i n}$ and $T$. In the third regime, $E_{k i n}$ and $T$ decouple and this coincides with the moment when the system becomes homogeneous again. In the literature, controversial statements can be found to the late time behavior of a cooling granular gas $[23,25,102,104,110,111]$. All of them find a change from the slope of Haff's law during the clustering process. In MD simulations it is common to measure the entire energy in the system, that means it is not possible to distinguish between kinetic energy caused by convective fluxes and thermal fluctuations. During the transition from HCS to ICS we find a change in the slope, too. This agrees with the results in [25], where a distinction between kinetic and thermal energy was also performed. From MD simulations, there are no data available in the long term of the ICS, which might be the case because of the density collapse which appears at large system sizes. We find that in the long time limit the ICS transitions back to a Haff's law like behavior, this transition has not been observed in MD simulations because of too short observation times. That the total energy follows a Haff-like behavior in the ICS agrees with the finding in [39] where the data for the viscoelastic spheres model were published. In addition we can observe that eventually the kinetic and the thermal energy couple and both follows Haff's law (for the model of constant $\varepsilon$, for viscoelastic spheres as an intermediate state). We could show this behavior for systems up to ten times larger than the critical system size (final data of the degree of clustering can be found in Fig. 7.2). It could be interesting in the future to study systems close to the critical system size with the help of MD simulation to compare with the present results.

\subsection{Clustering close to the critical system size}

Here we study more closely the clustering transition due to finite-size effects. We introduce the variable $\mathfrak{l} \equiv L / L_{\text {crit }}$ which is a length in terms of critical system sizes (see also the linear stability analysis in Chapter 2). We focus now on systems with $\varepsilon=$ const close to the $\mathfrak{l} \approx 1$ point. Figure 7.2 shows the density fluctuations at long times at $\varepsilon=0.9$ and $\varepsilon=0.999$ for different values of the dimensionless parameter $\mathfrak{l}$. We find a sharp transition at $\mathfrak{l} \approx 1$ from a homogeneous gas with no clusters to a system which exhibits clusters, as expected from linear stability analysis [35]. However, linear stability analysis cannot predict the asymptotic density and size of the clusters. We find that the asymptotic cluster densities are smaller than maximum packing fraction and their fluctu- 


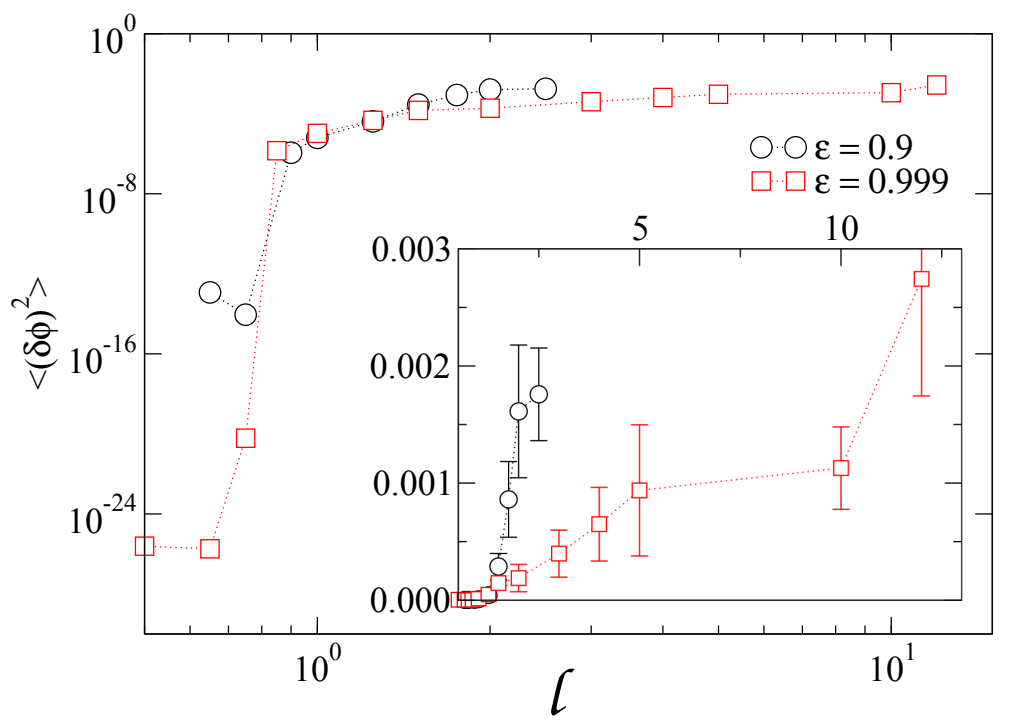

Figure 7.2: Asymptotic value of the fluctuations in the ICS as function of relative system size to the critical size $\mathfrak{l} \equiv L / L_{\text {crit }}$. For a fixed coefficient of restitution the fluctuations reach values which depend on the system size. The plot shows simulations for $\varepsilon=0.9$ and $\varepsilon=0.999$ and $\bar{\phi}=0.05$. The inset shows the same data on a linear scale.

ations exhibits system-size dependent, characteristic values (see also the attached movie freeCoollingCloseCritSize.avi). The inset of Fig. 7.2 shows that the growth of the clusters is not unbounded, but instead scales approximately linearly with the system size. Systems with a $\varepsilon$ closer to unity tend to form not as dense clusters as system with a lower $\varepsilon$ in terms of $\mathfrak{l}$. To the best of our knowledge, we report here for the first time that systems close enough to the critical system size cluster to an asymptotic packing fraction $\phi_{A}<\phi_{m}$, that is the system does not undergo a density collapse to the maximum packing fraction.

\subsection{Clustering in systems with solid walls}

We want to shortly discuss the situation of a granular gas cooling down within a box with rough solid walls. One might expect that clustering appears in any case due to the additional loss of energy caused by friction at the wall.

In Fig. 7.3 we see the evolution of a freely cooling homogeneous granular gas inside a box with rough walls (see the full movie attached under coolingInBoxClustering.avi). The clustering is only observed if the system size is above the critical system size $L_{\text {crit }}$ (see Section 2.9 and the attached movie coolingInBoxNoClustering.avi) otherwise the system stays in a HCS. Clusters are predominantly located in proximity to the walls. This is not surprising as the presence of friction may lead to the accumulation of matter at the walls. 


\section{(a)}
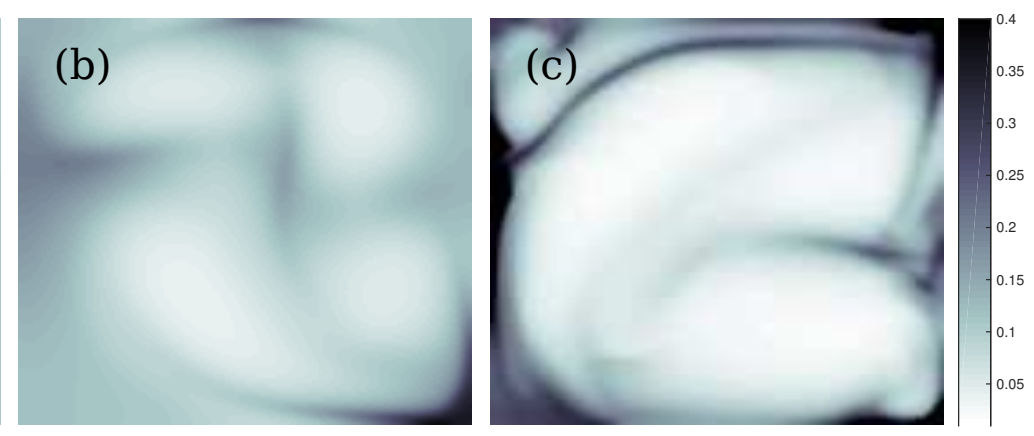

Figure 7.3: A freely cooling granular gas inside a box with rough hard wall. The friction coefficient on the wall is $C_{f}=0.9$ (compare initial condition in section 4.16). The system size is $1280 \sigma \times 1280 \sigma, \bar{\phi}=0.1$ and $\varepsilon=0.99$. We observe clustering along the walls only for systems larger than the critical system size. The system is about 4 times larger than $L_{\text {crit }}$.

\subsection{Cluster growth in the case of viscoelastic spheres}

Here we study in more detail the time till the clusters in the viscoelastic model disappear. We quantify the degree of clustering again (as in Section 5.1) by measuring the density fluctuations $\left\langle(\delta \rho)^{2}\right\rangle=\frac{1}{\overline{\bar{\phi}}^{2}}\left\langle(\delta \phi)^{2}\right\rangle$ across the system. Figure 7.4 shows the evolution of the density fluctuations for systems having the same initial $\varepsilon$ but differing in size. For constant $\varepsilon=0.99$ the density fluctuations reach a plateau value of about $5 \times 10^{-3}$. The density fluctuations for $\varepsilon=\varepsilon(T)$ also reach typical values, but only as intermediate states. They always decay to vanishing values, but with a characteristic time $t^{*}$ that depends on the system size. To avoid natural fluctuations in the degree of clustering we define $t^{*}$ as the time when $\left\langle(\delta \phi)^{2}\left(t^{*}\right)\right\rangle=10^{-20}$. Figure 7.5 shows the dependence of $t^{*}$ on the linear size of the system. Except for the smallest size investigated, the calculations are well fit by a power law with the rather large exponent of 12 .

We are now in a position to rationalize the finite-size dependence of $t^{*}$ with a heuristic argument in the following way. For short time intervals, the typical impact velocities will not change significantly. Hence, one can use the present value of $\varepsilon$ to define a timedependent $L_{\text {crit }}$ as in Eq. (1.2). It is useful to introduce the dimensionless quantity $\mathfrak{l} \equiv$ $\frac{L}{L_{c r i t}}$. Because $L_{\text {crit }}$ diverges as $\varepsilon \rightarrow 1, \mathfrak{l}$ vanishes. We are interested in the time $t^{\prime}$ when $\mathfrak{l} \approx 1$ which corresponds to the onset of declustering (see the transition to regime III in Fig. $7.1(\mathrm{~b}))$. We can then write $\mathfrak{l}=1=L\left(1-\varepsilon^{2}\right)^{1 / 2}$. To first order, the coefficient of restitution for viscoelastic particles depends on temperature as $\varepsilon=1-\left(1-\varepsilon_{0}\right) T^{1 / 10}$, where $\varepsilon_{0}=\varepsilon(t=0)$ and $\gamma=1-\varepsilon_{0}$. Keeping only terms of first order in $T^{1 / 10}$, the condition $\mathfrak{l} \approx 1$ leads to the relation $L^{-2} \approx\left(1-\varepsilon_{0}\right) T^{1 / 10}$. We know from Fig. 7.1 that Haff's law holds also in the ICS. We can then use its large time approximation for the case of viscoelastic particles $T \approx t^{-5 / 3}$. Putting all together we find the power law

$$
t^{\prime} \propto L^{12} .
$$




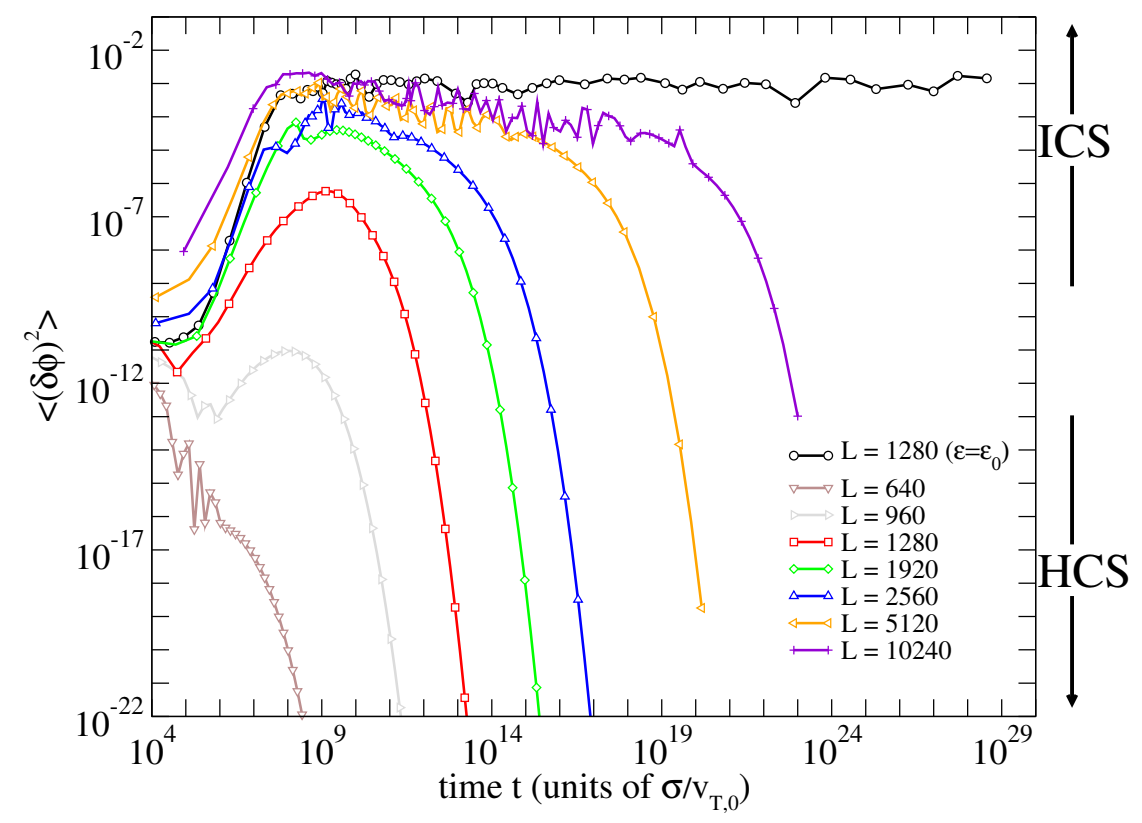

Figure 7.4: Temporal evolution of density fluctuations in 2D granular gases with an average filling fraction $\bar{\phi}=0.05$. The black circles correspond to calculations at constant $\varepsilon=0.99$. All other symbols correspond to calculations for viscoelastic particles where $\varepsilon_{0}=\varepsilon(t=$ $0)=0.99$. The critical system size for this $\varepsilon_{0}$ is $L_{\text {crit }} \approx 500$. If the system is large enough, the fluctuations grow till the clustering becomes visible. For a constant $\varepsilon$ the fluctuations reach a plateau. When $\varepsilon=\varepsilon(T)$ the fluctuations first grow to a size dependent maximum but eventually vanish after a certain time. This time grows with system size.
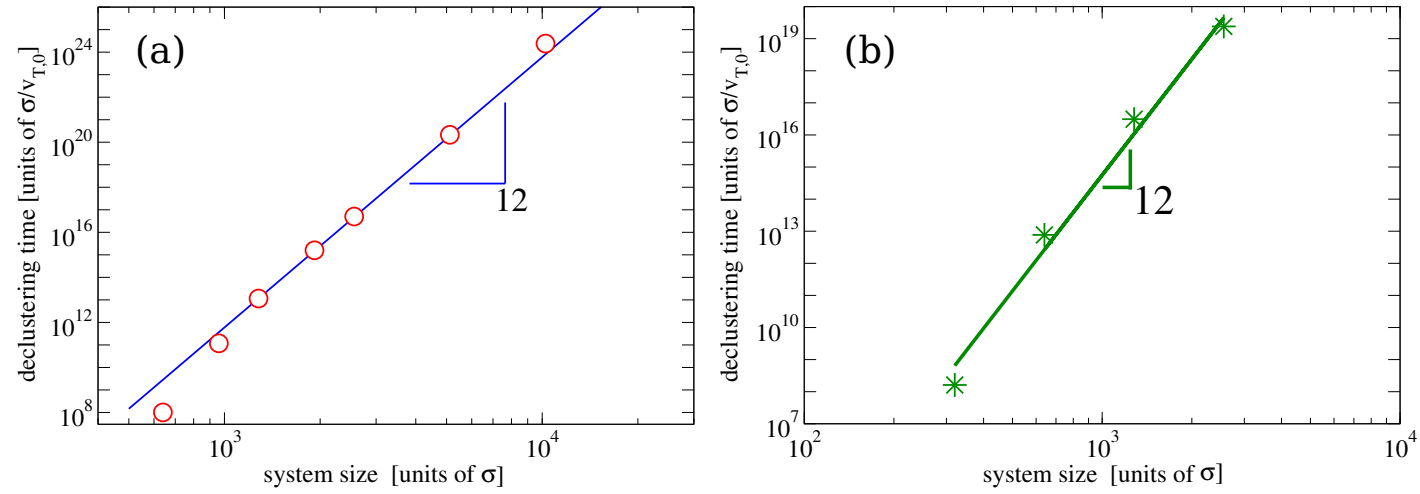

Figure 7.5: The time $t^{*}$ characterizing the disappearance of clusters grows with the size of the system in 2D (panel (a)) and in 3D (panel (b)). Results from DNS calculations are shown as circles. The solid line represents a power law with exponent 12 . The value of $t^{*}$ for the smallest $L$ does not fall on the power law because the ICS is not fully developed (compare with Fig. 7.4). 
Because the late-time evolution of $\left\langle(\delta \phi)^{2}\right\rangle$ is well approximated by a power law (see Fig. 7.4) we can safely conclude that $t^{*} \propto t^{\prime}$, which then matches our DNS calculation in Fig. 7.5. Equation (7.1) implies that the declustering transition for viscoelastic particles has no typical time scale, but is instead a finite-size effect.
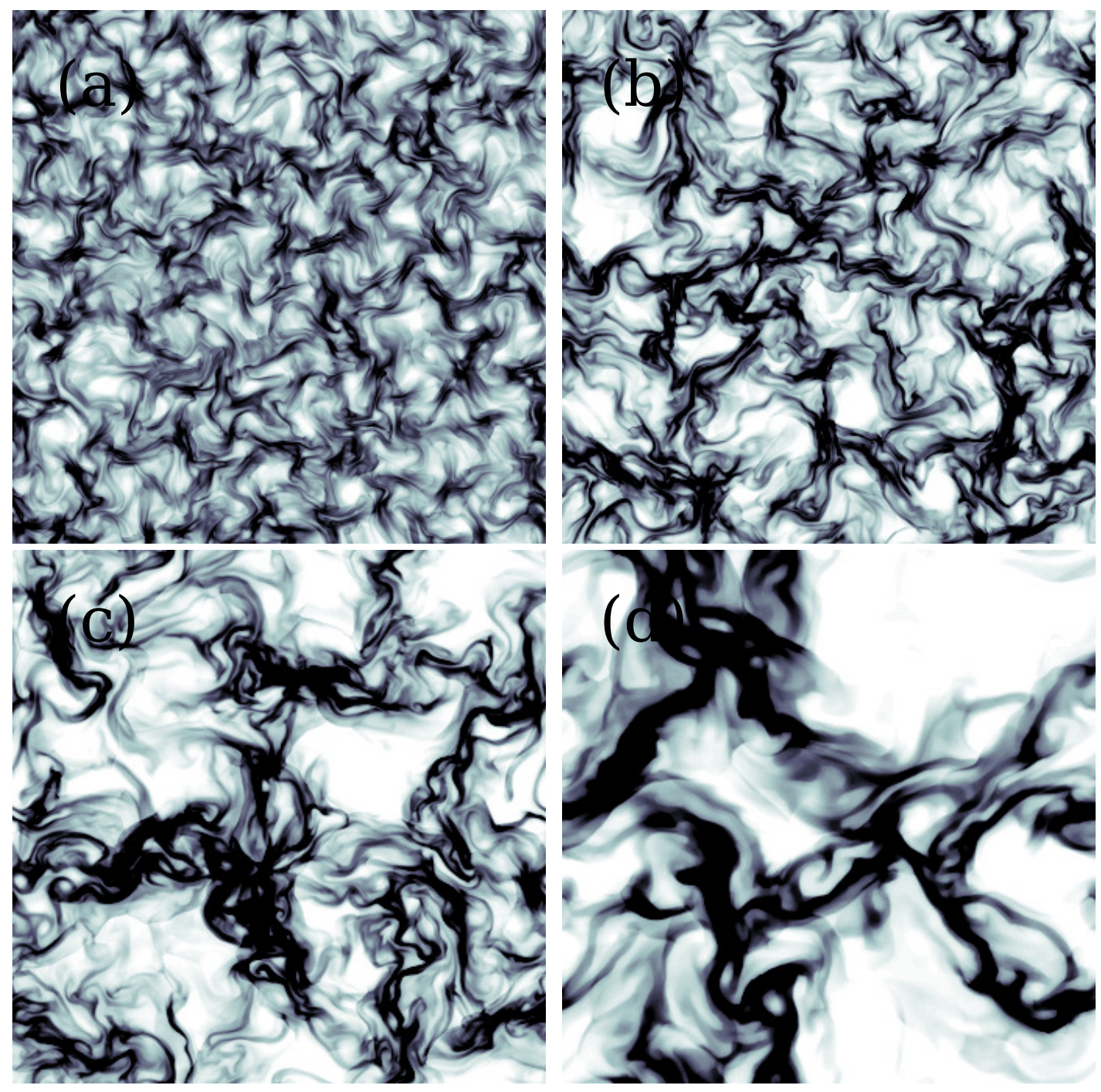

Figure 7.6: Evolution of a freely cooling granular gas with viscoelastic spheres at time $t=3.7 \cdot 10^{8}(a), t=1.3 \cdot 10^{9}(b), t=6.3 \cdot 10^{9}(c), t=1.4 \cdot 10^{11}(d)\left(t\right.$ in units of $\left.\left[\frac{\sigma}{v_{t h, 0}}\right]\right)$. The cluster form and growth till they reach system size (see the full movie attached under viscoelasticCooling.avi).

Fig. 7.6 shows the evolution of a freely cooling granular gas for the viscoelastic spheres model. We can observe the growing of the cluster till they reach system size.

To characterize the clusters we show their typical length scale $\lambda=\frac{\sum_{k} S(k)}{\sum_{k} k S(k)}$ in Fig. 7.7. The question if the declustering can appear before the size of the clusters reach system size is of fundamental importance to the question if the declustering is a finite-size effect. Comparison of Fig. 7.7 and Fig. 7.4 reveals that the disappearance of clusters occurs not before the characteristic fluctuation size reaches 0.5. That means that declustering does 


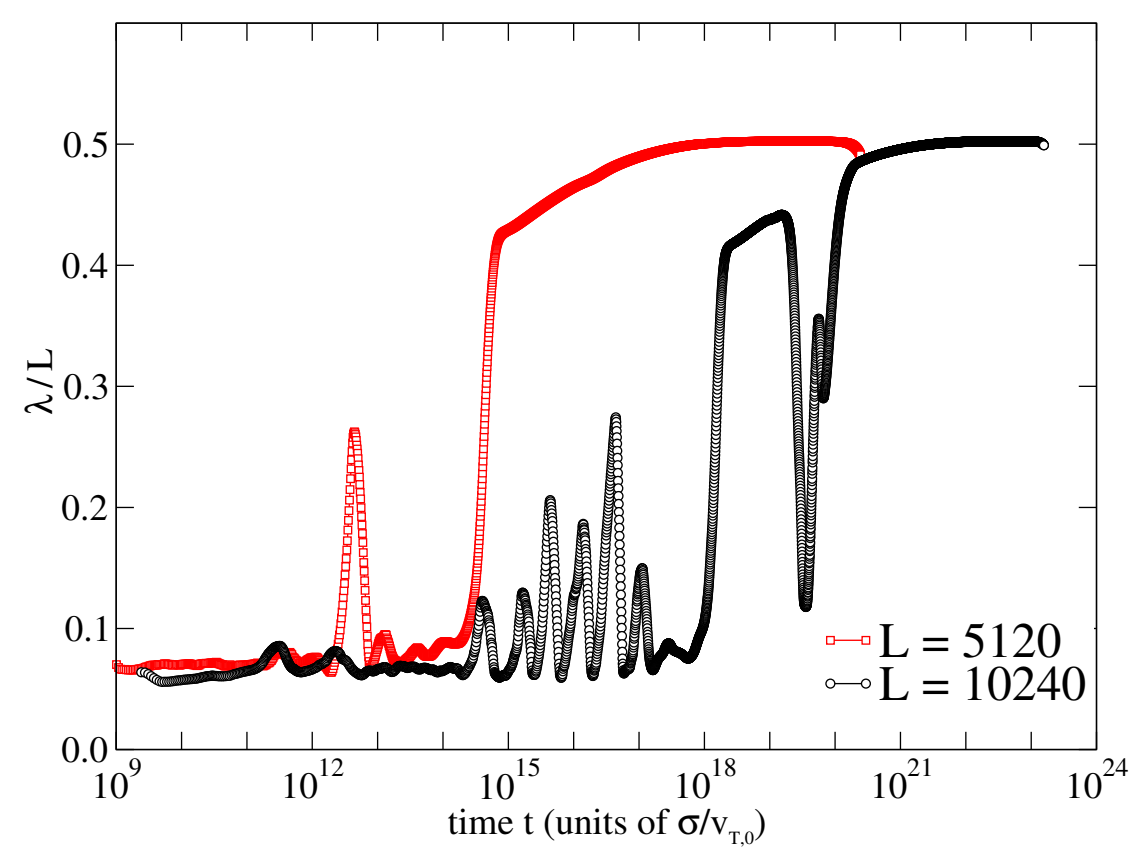

Figure 7.7: Typical length of the clusters during the inhomogeneous cooling process for $\varepsilon(t=0)=0.99$ and $L=5120$ and $L=10240$. The values fluctuate and grow till values above 0.5. The time at which this value is reached corresponds to the disappearing of clusters in the system (see Fig. 7.4). We calculate the length scale $\lambda$ from the first moment of the Fourier transform of the density correlation function.

not occur before the size of the clusters reaches the order of the system size. From MD simulations and linear stability analysis of the hydrodynamic equations it is known that in a granular gas with $\varepsilon=$ const the clusters grow in size until they reach the size of the system $[23,24]$. For variable $\varepsilon$ the same behavior has been observed in MD simulation, if Eq. (1.3) holds for any $u$ [39]. In a simplified model where $\varepsilon=1$ for impact velocities below a threshold value the clusters dissolve before reaching system size [110, 111, 115]. However, hydrodynamic simulation in [39] show a cluster growth till system size for variable $\varepsilon$ as well.

In summary, we have shown the finite-size character of the decaying of clusters in a freely cooling granular gas of viscoelastic particles. We clarified that the disappearing of density fluctuations does depend only on the size of the system. The decay time follows such a strong power law, $t^{\prime} \sim L^{12}$, that only in systems close to the initial critical system size it is realistically possible to observe both the clustering and declustering transitions. Thus, for any amount of dissipation $\left(1-\varepsilon^{2}\right)$ in the system, one can find a system size at which the clusters will appear as stable for an exceedingly long time. On the other hand, our results show that close to $\mathfrak{l} \gtrsim 1$ the degree of clustering is extremely week (see Fig. 7.2), which poses the challenge to find a system size that is large enough to observe the transition from HCS to ICS, but also small enough to observe the second transition (from ICS back to HCS) on a realistic time scale. Finally, these results indicate that an infinitely large system will not decluster. 


\section{Extensions and Outlook}

In this last chapter we highlight the extension of applicability and effectiveness of our DNS for granular fluids. We point out interesting future projects which could be studied within a hydrodynamic framework. In the first Section we describe the distribution functions which we measure in the HCS and the ICS. Then we discuss driven granular systems with solid walls. We show that it is possible to shake a system in such a way that the density field becomes homogeneous again. In Section 8.3 we demonstrate the present of the granular Leidenfrost effect and convection rolls in granular Hydrodynamics. Here we give a phase space of the regions where we can find the different states and compare this to results studied in MD simulations. In a more realistic model we have to assume the coefficient of restitution follows more a distribution than a fix parameter. We discuss the effects such a noisy $\varepsilon$ has to a granular system. Eventually we show results from our DNS under the influence of a central force.

\subsection{Distributions of density, momentum and temperature}

A cooling granular gas is far away from an equilibrium state, thereby we cannot assume that the probability density functions of quantities such as velocity follows a Gaussian distribution. In the past decades a lot of studies have focused on this field [30, 102, $111,116]$, especially in the homogeneous cooling state. It is expected and observed from MD simulations that the velocity distribution is nearly a Gaussian with "high velocity tail" corrections. This overpopulation was first predicted in 1997 [117]. The decay of the distribution changes from a Gaussian distribution $\sim \exp \left(-c_{1} v^{2}\right)$ to an exponential decay $\sim \exp \left(-c_{2} v\right)$, where $c_{1}$ and $c_{2}$ are constants. Therefore much more particles are found at high velocities than in a comparable hard sphere gas.

In Fig. 8.1(a) the probability distribution function of the momentum is shown. It is very close to a Gaussian distribution (red dashed line) and does not show the expected effect of overpopulated long tails. But we have to keep in mind that each point represents 

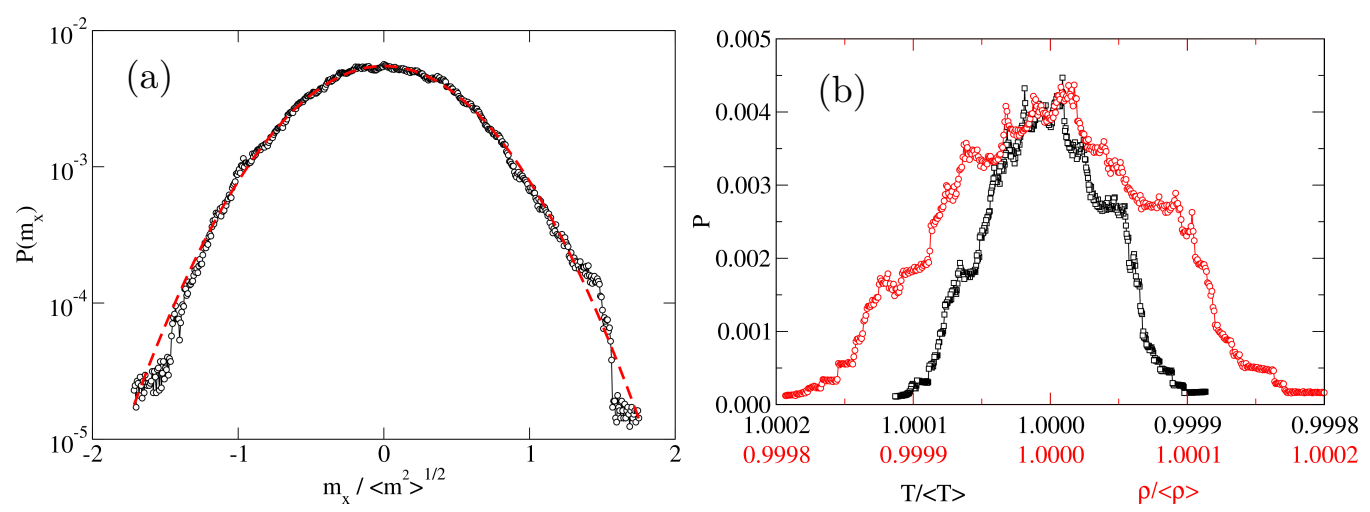

Figure 8.1: Distribution of the momentum (a), density and temperature (b) in the HCS. The system has a size of $(25600 \sigma)^{2}, \bar{\phi}=0.01$ and $\varepsilon=0.9$ and is divided into $1024^{2}$ finite volumes. The distribution is calculated at a temperature $\langle T\rangle \approx 10^{-12}(T(t=0)=1)$; the convective velocities are 4 orders of magnitude below the thermal velocities.

the average value inside a finite volume (see also the finite volume method in Section 4.4). That means that we do not measure the momentum distribution but the distribution of the average momenta inside the system. The central limit theorem [118] states that the arithmetic mean of a sufficiently large number of independent random variables sampled from the same distribution will follow a Gaussian distribution. This result is what we expect when the velocity distribution function $f(\underline{\mathbf{r}}, \underline{\mathbf{u}})=f(\underline{\mathbf{v}})$ (see also the Boltzmann equation in Section 2.5) is independent from space. Figure 8.1(b) shows the distribution of the temperature and the density in the HCS. We can conclude that the distribution is not a Gaussian distribution, which means that the fluctuations in $\rho$ and $T$ do depend on space. We already know from the results of Section 5.3 that $\rho$ and $T$ are anti-correlated. In fact, careful inspection of Fig. 8.1(b) reveals that where $T$ exhibits a local maximum, $\rho$ exhibits a local minimum and vice versa.

In the ICS the situation is totally different. The distributions of momentum, density and temperature change drastically to non-Gaussian distributions and we can state that the quantities in the finite volumes $i$ and $j$ do not follow anymore the same distribution, which means that the central limit theorem does not hold here. Figure 8.2 displays the distribution in the ICS. The momentum distribution has changed to a nearly Gaussian distribution with overpopulated "long tails". It seems interesting that the distribution of the convective momentum $P(\rho v)$ in the ICS looks like the momentum distribution $P(\rho u)$ which we would expect for the particles in the HCS; here $u$ is the particle velocity and $v=\langle u\rangle$ is the convective velocity. In the ICS the total energy is dominated by kinetic energy, which is the energy associated with the convective fluxes. One could argue that now the clusters themself behave like quasi-particles. This would heuristically explain why they follow a similar distribution to the grains in the HCS. We leave the task of understanding the origin of these distribution for future research. 

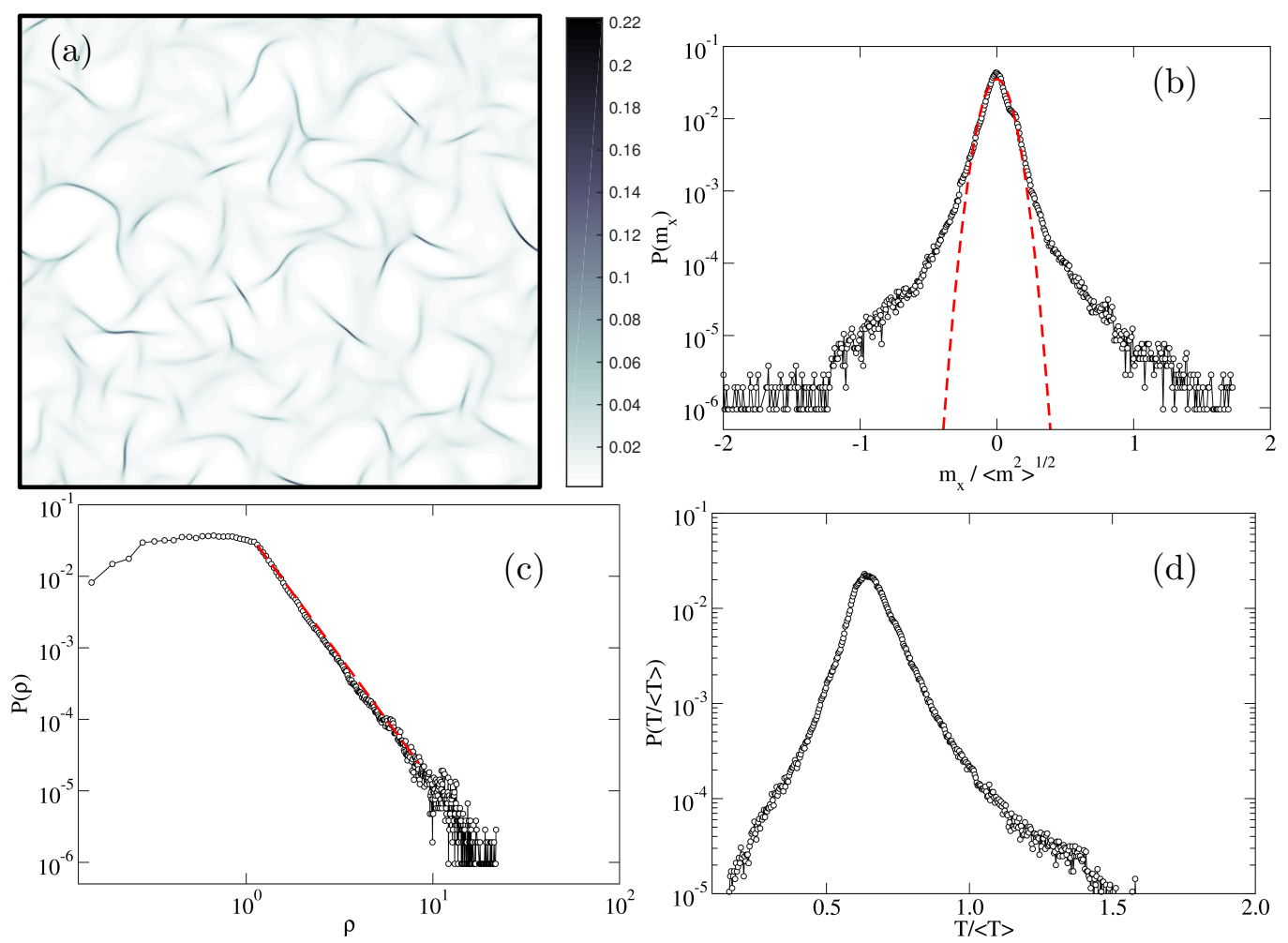

Figure 8.2: (a) Snapshot of the system from which various probability distributions were calculated. Distribution of the momentum (b), density (c) and temperature (d) in the ICS. The system has a size of $(25600 \sigma)^{2}, \bar{\phi}=0.01$ and $\varepsilon=0.9$. The power law fit in (c) is $y(x)=0.0439 x^{-3.52}$. 


\subsection{The generation of a homogeneous, steady state in granular gases from an inhomogeneous state}

We have studied the transition from HCS to ICS in very detail so far. Another interesting question arises when we think about the realization of a homogeneous granular gas. How is it possible to create a granular gas which has a nearly homogeneous temperature and density in every point of space? This question becomes much more relevant if we consider an experimentalist who tries to study the free cooling $[14,15]$. The injection of energy is typically done via shaking of walls. But within the context of low gravity experiments various problems appear. To realize a granular gas we need an energy transport from the boundaries to the center of the system. The shaking can lead to a concentration of grains in the center, then the energy flux breaks down because there is no longer physical connection between the grains and the walls. An inaccurate shaking protocol can also lead to a dense cluster which evolves in the system. Of course an important aspect is the system size. If the system is small (compared to the diameter of a particle) it seems to be easy to obtain a homogeneous gas via strong shaking, but other effects like the liquid-gas phase separation and convection rolls might also appear. It turns out that not only the geometry, the shaking amplitude or frequency play an important role but also transient processes are very important. This interesting topic was studied within the Bachelor's thesis of Marius Herr [119]. The thesis studied this problem within the hydrodynamic approach of granular gases (see Chapter 3) and the simulation were performed with the DNS solver we introduce in Chapter 4. We only give the final results here and refer the reader to the original work in [119]. An important point is that we are interested in systems which are large enough to show clustering (see Section 2.9), that means that the system must be larger than the critical system size in Eq. (2.89). In the beginning of a shaking process the grains close to the wall are accelerated to the center. This leads to a dilute area at the boundary. If the shaking starts too suddenly a big layer of grains detaches from the wall and forms a denser cluster evolving in the system. That is why the shaking should start with a smooth transient. Once a homogeneous gas is formed it is also necessary to turn the shaking off slowly again, otherwise a final convective flux might be left in the system and will destroy the homogeneous state again (see also Section 6 for the influence of convective flows). Details on the shaking parameters can also be found in [119]. It was expected that shaking from two directions leads to better results. This behavior was also confirmed within the Bachelor's thesis.

Figure 8.3 shows the dissolving of initial inhomogeneities for two different initial conditions. Figure 8.3 are taken from Bachelor thesis's of Marius Herr [119]. The work of Marius proved that it is possible, from a hydrodynamic point of view, to reach a nearly homogeneous granular gas out of an inhomogeneous granular gas. 


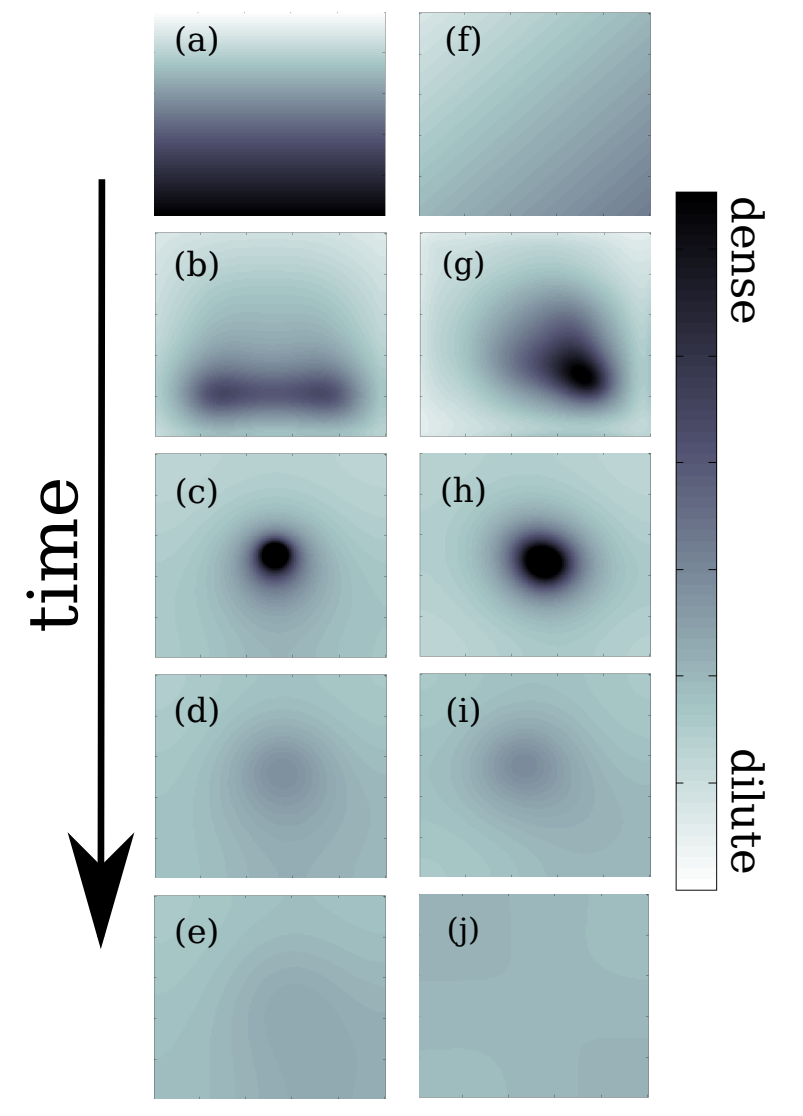

Figure 8.3: Creation of a homogeneous granular gas via shaking of the system in the $x$ - and $y$ - direction. Two different initial configurations are shown. The frequency is $f=20 \mathrm{~Hz}$, the ampliutude $A=0.14 \sigma$ and the system size, $L=285 \sigma$. The pictures are taken after 0,140,760, 2940, 6940 cycles ((a) - (e)) and after 0,160,300,1280, 7340 cycles ((f)-(j)). 


\subsection{Driven granular systems}

Granular materials are dissipative systems. That means that unless there is a mechanism of energy injection the energy of the system will eventually reach zero. Therefore, to study nonequilibrium steady states of granular materials an energy injection mechanism has to be devised. It is common to place a granular system within a vessel and vibrate the latter in one direction. The hydrodynamic equivalent of vibrating walls corresponds to an external force which results in a nonvanishing $\boldsymbol{a}$ in Eq. (3.24). The force $\boldsymbol{a}$ will in general depend on time; for our implementation we use a sinusoidal with a frequency $\nu$. Driven granular media show an enormous range of different structure formations [2]. We find in driven systems numerous phenomena which are known from molecular fluids, for example the Leidenfrost effect [120]. When a liquid droplet is in contact with a surface at a temperature larger than the liquid's boiling point a vapor layer quickly develops between the two phases. This vapor layer effectively insulates the droplet for some time. The Leidenfrost effect has been found also in granular materials [120]. This fact demonstrates that under suitable conditions a granular system behaves like a fluid. Therefore, driven granular media have attracted wide interest in the last decades $[1,46,47,114]$. In principle, such systems are quite easily accessible for experimentalist, but also with MD simulations. From the hydrodynamical point of view, the situation is very different. On the one hand, driven granular media typically have quite a large Knudsen number in the direction of the shaking, and on the other hand the system exhibits really strong gradients of the fields which makes the NS approach less valid. In addition, it is rather challenging to stabilize the numerical solver in such extreme situations. Therefore, we can conclude that driven granular systems are at the boundary of applicability of hydrodynamic predictions. Nevertheless, we have also studied driven granular system, to compare the possible results from hydrodynamics.

In Fig. 8.4 the field map for the density of a 2D, driven system exhibits structure formation that resembles convective rolls (see also the attached movies convectionRolls.avi and convectionRollsVorticity.avi). Figure. 8.4 (d)-(f) show experimental data for comparison. More details to the phase space in experiments can be found in [47].

Figure 8.5 displays the $\bar{\phi}-F$-phase diagram and the different steady states observed. We find three collective states: $(i)$ the bouncing bed, $(i i)$ the granular Leidenfrost effect, and (iii) convection rolls. The bouncing bed state corresponds to uniform, dense layer oscillating in the shaking direction. The Leidenfrost effect and the convection rolls are states qualitatively similar to the analogues in molecular fluids.

Even if Fig. 8.5(b) depends in addition on the amplitude of shaking, we can conclude that our hydrodynamical model can reproduce the phenomenology of driven granular systems that has been found in experiments and MD simulations. These results give us even more confidence in the results of our numerical solver. We observe a transition regime (green squares in Fig. 8.5) where the Leidenfrost effect and the convection rolls coexist in 

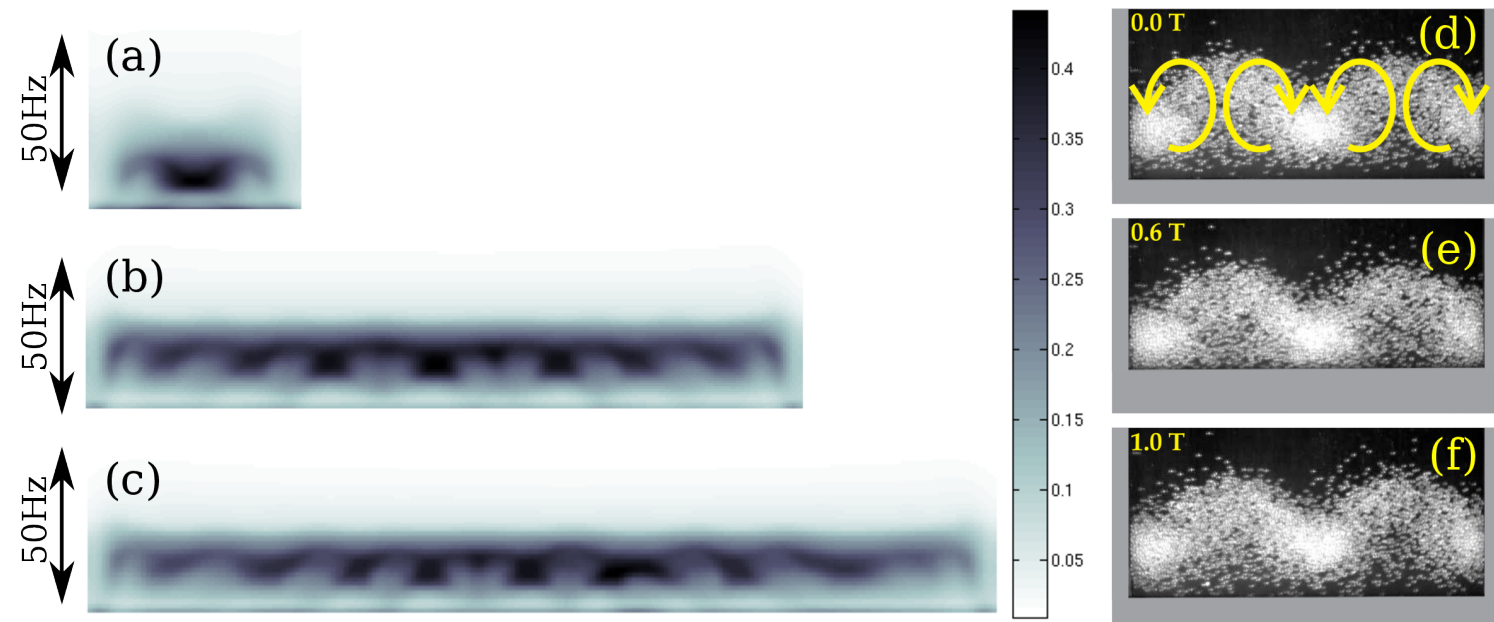

Figure 8.4: Steady state of driven granular media. The systems are driven in the vertical direction with a frequency of $50 \mathrm{~Hz}$ and a strength of $\Gamma=12 \mathrm{~g}$. In addition there is constantly $1 g$ gravity force acting on the system in the downward direction. The systems contain hard wall boundary conditions on each side with a friction strength of $C_{f}=0.9$ (see Section 4.16 for details on boundary conditions). The system size is $256 \sigma \times 256 \sigma$ in (a), $461 \sigma \times 115 \sigma$ in (b) and $630 \sigma \times 115 \sigma$ in (c). (d)-(f) are results from experiments; the pictures are taken from Eshuis et al. (2007) [1].

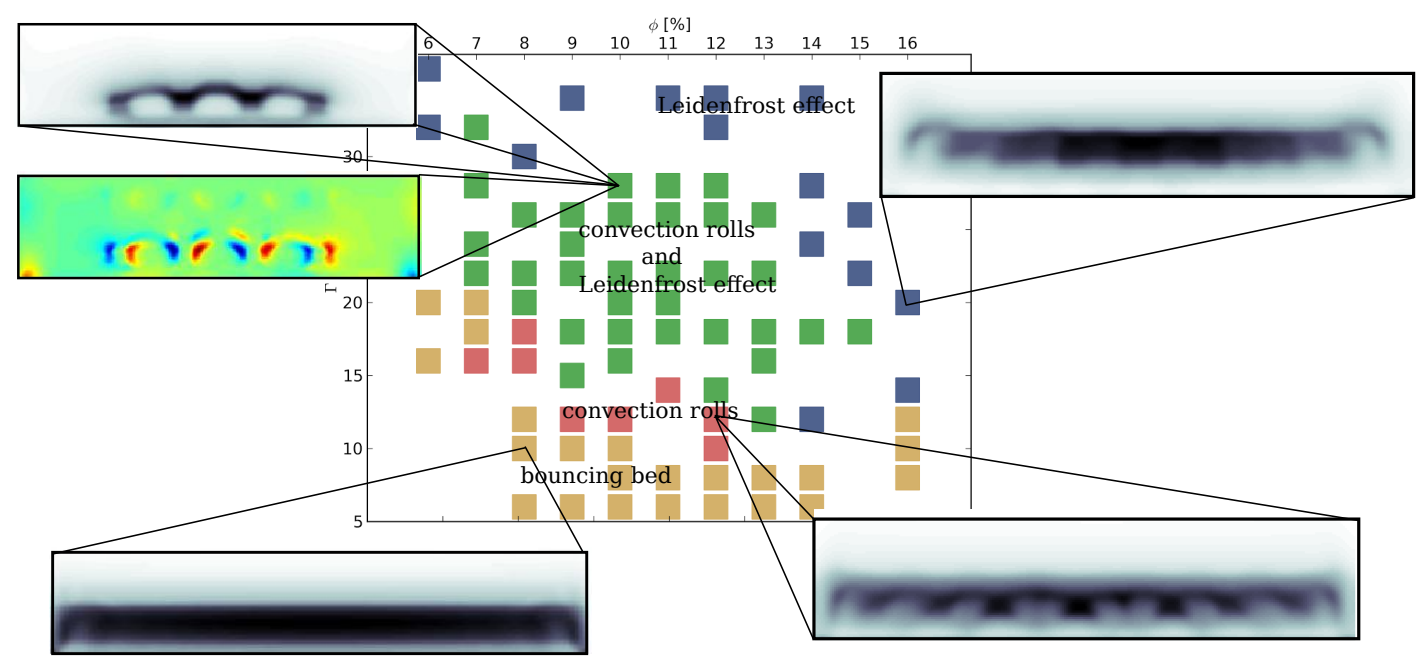

Figure 8.5: $\bar{\phi}$-F-phase diagram of driven granular gases. We highlight some representative states with the field maps of the density. The normalized force $\Gamma$ is measured in terms of the magnitude of the external force. The color map for the convection rolls displays the vorticity field of the granular gas. Red (blue) color indicates positive (negative) vorticity. 
a superposition, that means that the convection rolls do not touch the ground anymore (see the attached movie convectionRollls.avi). The inset of the phase-diagram shows the vorticity $\nabla \times(\rho \boldsymbol{v})$. From the vorticity plot we can see that we have indeed convection rolls in the system. Our findings agree rather well with the experimental data in [47]. Finally, we note that we do not observe the undulation phase of driven granular gases. This is interesting and could simply mean that the undulation phase is a non-hydrodynamic effect which has its origin in the particle character of the grains.

The results shown here arose from the contribution of Joscha Tabet who studied driven granular systems during his Bachelor's thesis. He performed the simulation and we are very thankful for his vigorous efforts to find the correct system parameters to run stable simulations which gave such nice results. For more details we refer the Bachelor's thesis in [121]. Finally the work presented here proves that the hydrodynamic approach can reproduce physical effects even in the case of extreme forces and gives us even more confidence in the approach and the results of our solver. To the best of the authors knowledge we present here the first report of the granular Leidenfrost effect and the granular convection rolls within a hydrodynamic granular theory. We hope that future work can lead to an even better understanding of the structure formation in driven granular medias.

\subsection{Stochastic coefficient of restitution}

We have so far studied two different models to describe the behavior of a granular gas: $(i)$ the model of constant coefficient of restitution, and (ii) the model of viscoelastic spheres. It is legitimate to ask whether or not these simplified models can capture the nature of a granular gas. A more realistic model has to take into account that not all particles are equal, that is, some of them might have disparate surface shapes and roughness, some might not be perfect spheres and have rotational degrees of freedom and particles might also carry electric charges [48]. The coefficient of restitution is thereby influenced by a number of parameters and physical conditions even if the particles are made of the same material and are of nearly equal size. If we consider a large number of collisions in a granular fluid, it is then easy to imagine that the values of coefficient of restitution in the individual collisions have a certain distribution of probability, rather than being fixed at a certain value (whether or not depending on the temperature). Experimental and numerical work to characterize this distribution was performed in [49, 122].

To model the unknown influences on the coefficient of restitution we added a noise to the local $\varepsilon$, so that we can study the influence of small local random changes on the behavior of cooling granular gases. We studied the influence of three different distributions: $(i)$ an uniformly distributed, (ii) Gaussian distributed, and (iii) Laplace distributed coefficient of restitution with the mean value $\varepsilon_{M}$ and a standard deviation of $\sigma_{\varepsilon}$. We adapt the 
random distribution locally to each finite volume at each time step. During a time step the $\varepsilon$ does not change. For the uniform distribution we calculate the $\varepsilon$ as follows

$$
\varepsilon=\varepsilon_{M}\left(1+\eta_{N} \zeta\right),
$$

where $\eta_{N}$ is the noise strength and $\zeta$ is a uniformly distributed random variable in the interval $[-1,1]$. In the case of a normal distribution in Eq. (8.1), $\zeta$ becomes a normal distributed variable with mean $\zeta_{M}=0$ and a standard deviation $\sigma_{\zeta}=1$. The Laplace distribution is a combination of two exponential distributions following the probability distribution

$$
p(\varepsilon)=\left\{\begin{array}{c}
\mathrm{e}^{s_{1}\left(\varepsilon-\varepsilon_{M}\right)}, \varepsilon<\varepsilon_{M} \\
\mathrm{e}^{s_{2}\left(\varepsilon_{M}-\varepsilon\right)}, \varepsilon>\varepsilon_{M},
\end{array}\right.
$$

where $s_{1}, s_{2}$ are different slopes of the distribution. The experimental data in [49] suggest a Laplace distribution for the coefficient of restitution for viscoelastic spheres. In Fig. 8.6 we
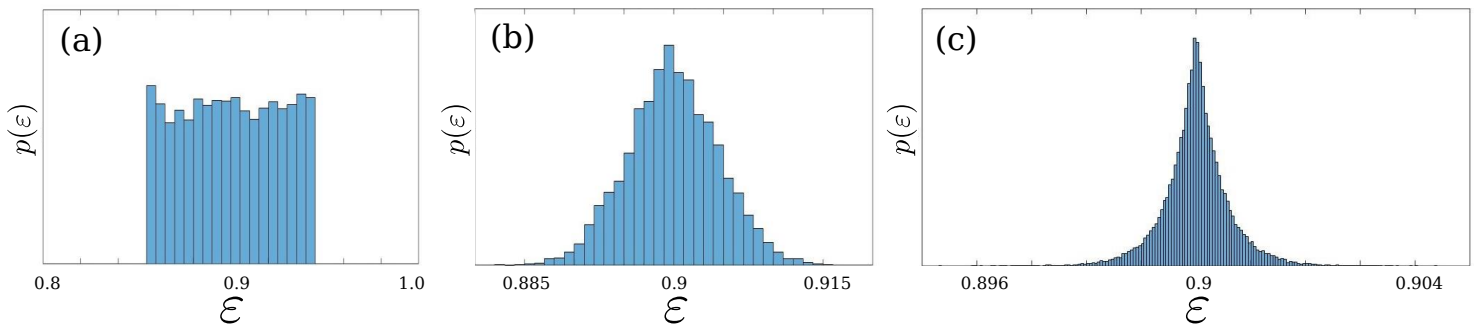

Figure 8.6: Example data for a stochastic variable which follows a uniform distribution (a), a normal distribution (b) and a Laplace distribution (c).

see an example of the three different implemented distributions, the data were calculated within our DNS.
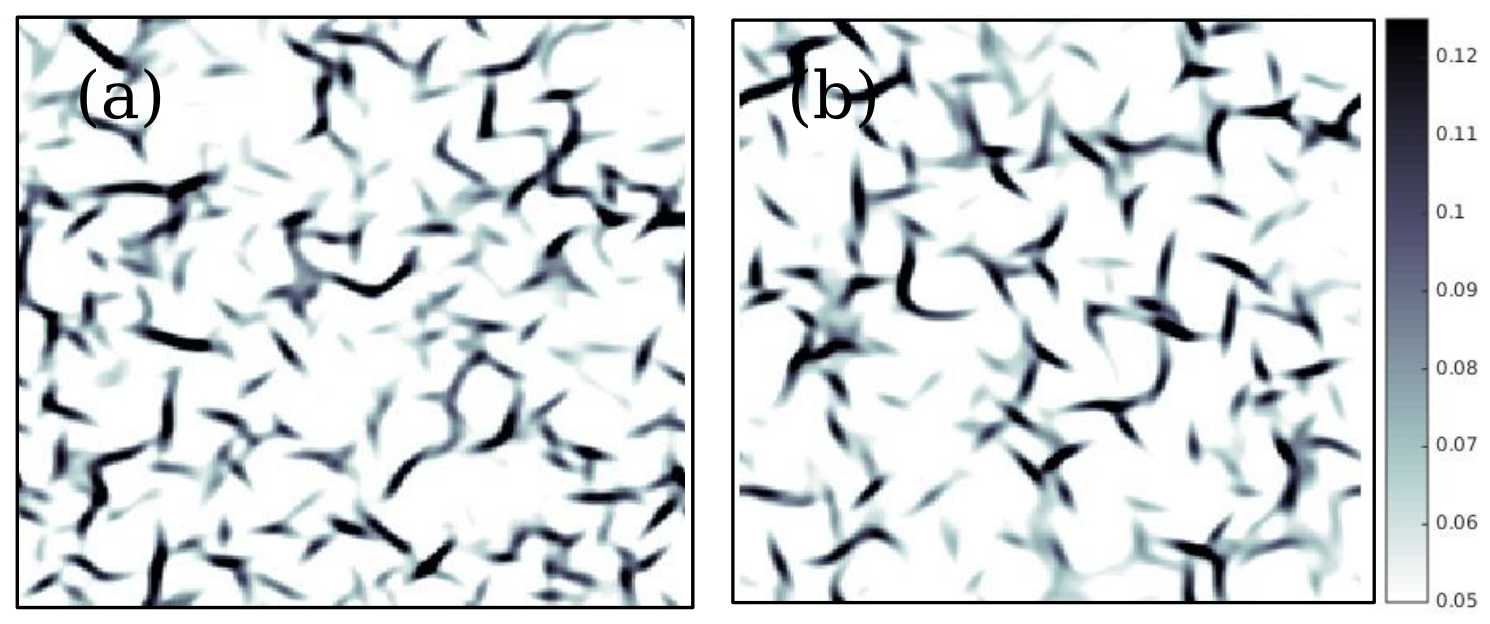

Figure 8.7: Shape of dense clusters after the transition from HCS to ICS. The filling fraction is $\bar{\phi}=0.05$. (a) Results for a system whit constant $\varepsilon_{M}=0.9, \sigma_{\varepsilon}=0$. (b) Results for a system with a distribution of $\varepsilon$ with the $\varepsilon_{M}=0.9, \sigma_{\varepsilon}=0.09$. 
We mainly focus on two characteristics here: $(i)$ the cluster size at the transition from HCS to ICS and (ii) the cooling behavior of the systems. We discussed in Section 5.4 that the cluster formation depends on the value of $\varepsilon$ and $\phi$. In Fig. 8.7 we see the structures forming in the ICS of a freely cooling granular gas with stochastic coefficient of restitution. We observe neither a qualitative change in the shape of the clusters nor a quantitative difference in the number or size of the clusters. Figure 8.7(b) shows the results for system where $33 \%$ of the local $\varepsilon$ is already above unity. The system does still behave as if it were determined by its mean value $\varepsilon=0.9$. That means that clustering behavior is qualitatively unmodified by adding noise to the constant $\varepsilon$ model.
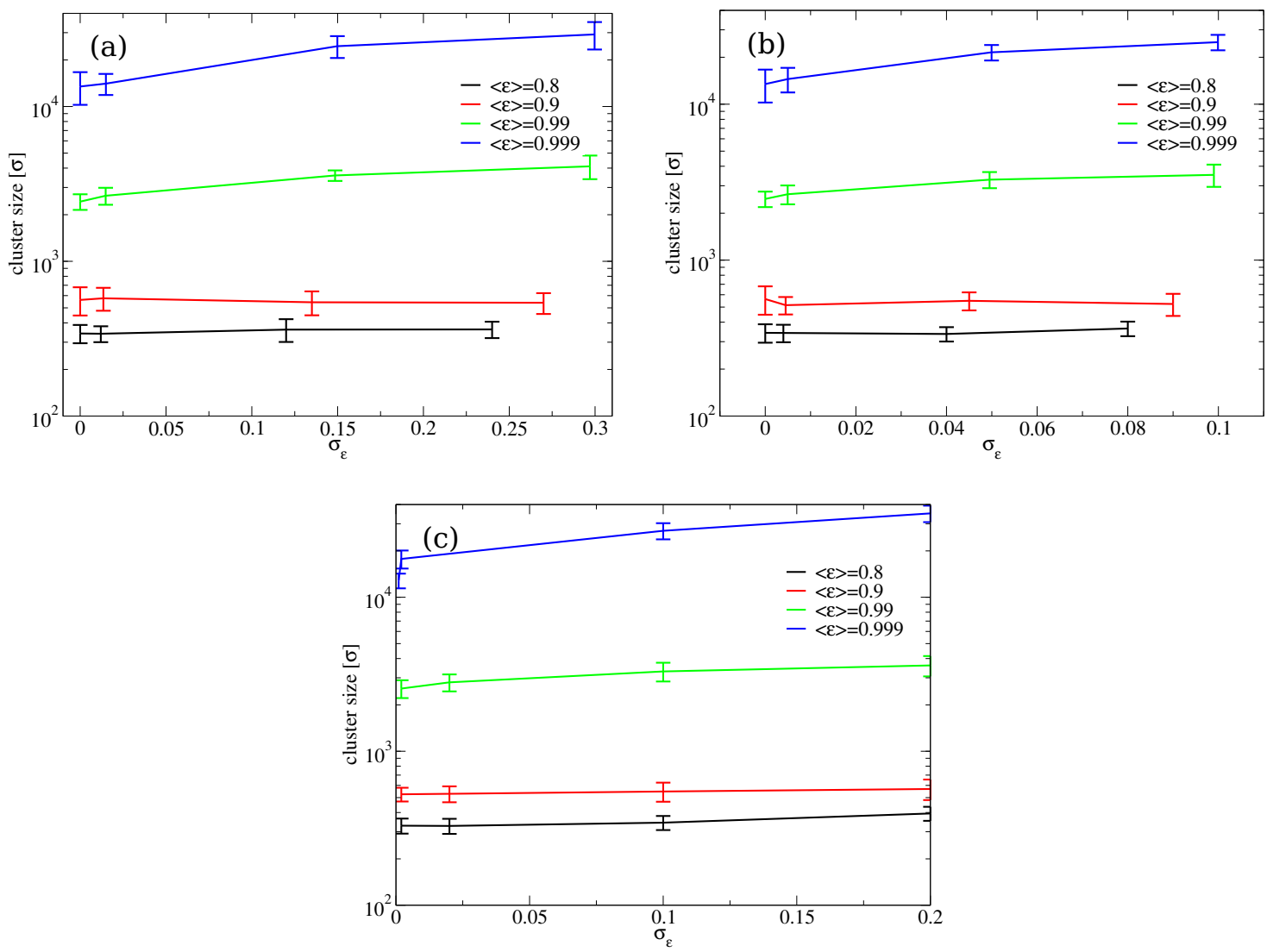

Figure 8.8: Dependency of the typical cluster size on $\sigma_{\varepsilon}$ for different distributions after the transition from HCS to ICS; (a) uniform distribution, (b) normal distribution, (c) exponential distribution.

Figure 8.8 sketches the dependency of the cluster size on the standard deviation for different distributions. We cannot observe any influence of the functional form of the distribution. The standard deviation of the distribution $\sigma_{\varepsilon}$ does not effect the system very much, only for $\varepsilon$ close to unity we find an influence of $\sigma_{\varepsilon}$ : the clusters become larger as $\sigma_{\varepsilon}$ grows.

Figure 8.9 displays the cooling behavior of a simulation with a noisy $\varepsilon$. From the temperature evolution, we are not able to see any influence of a noisy $\varepsilon$. We do not find any deviation from Haff's law for all observed distributions and different $\sigma_{\varepsilon}$. This 


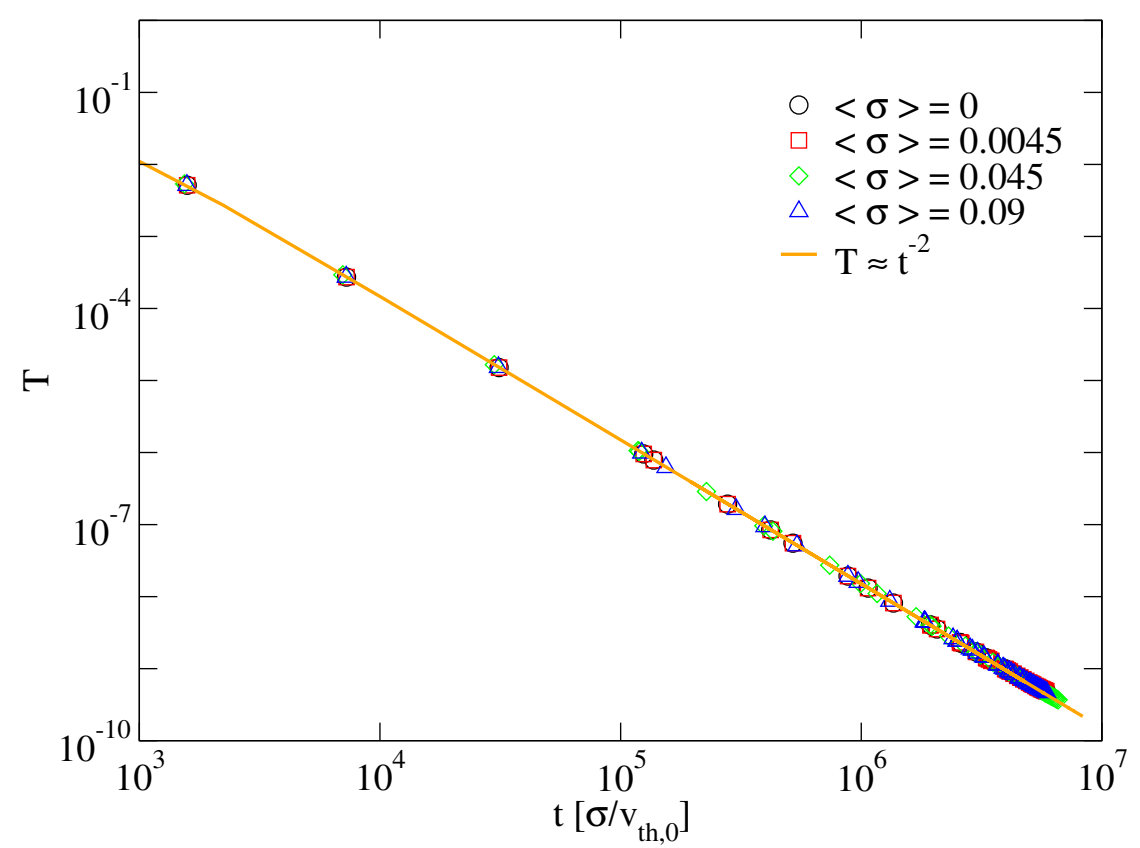

Figure 8.9: Temperature evolution for system with different $\sigma_{\varepsilon}$ in the normal distribution; $\varepsilon_{M}=0.9, \bar{\phi}=0.05$. The orange line marks Haff's law.

is interesting due to the fact that as $\sigma_{\varepsilon}$ increases more and more local values of $\varepsilon$ may become larger than unity, but, on the other hand, we also find more dissipative regions then.

We conclude here that the dynamics of a free cooling granular gas is drastically dominated by the average $\varepsilon$. This means, furthermore, that the simple model of a constant $\varepsilon$ is even more robust than one might expect in the first place and this gives finally more confidence that our results reproduce physical phenomena. We gratefully thank Samkit Shah for his contribution to the results of this section, his report can be found in [? ]. He supported us by carrying out the DNS and analyzing the necessary data within a three month Ludwig Prandtl internship at the Max Planck Institute for Dynamics and Self-Organization.

\subsection{Central forces}

In this last section we show the results from our DNS that include central attractive forces. One phenomenon where the behavior of granular gases might be important are protoplanetary discs. Still nowadays it cannot be conclusively clarified what seeds the formation of planetoids in a protoplanetary disc. Even in the densest areas of such discs only about $1 \%$ of the mass is of granular nature, the other $99 \%$ are gases especially hydrogen and helium. Nevertheless it might be possible that the remaining grains still can be described within the context of hydrodynamic granular fluids context and might lead 
to some contribution in the understanding of this field. Figure 8.10 displays the collapse
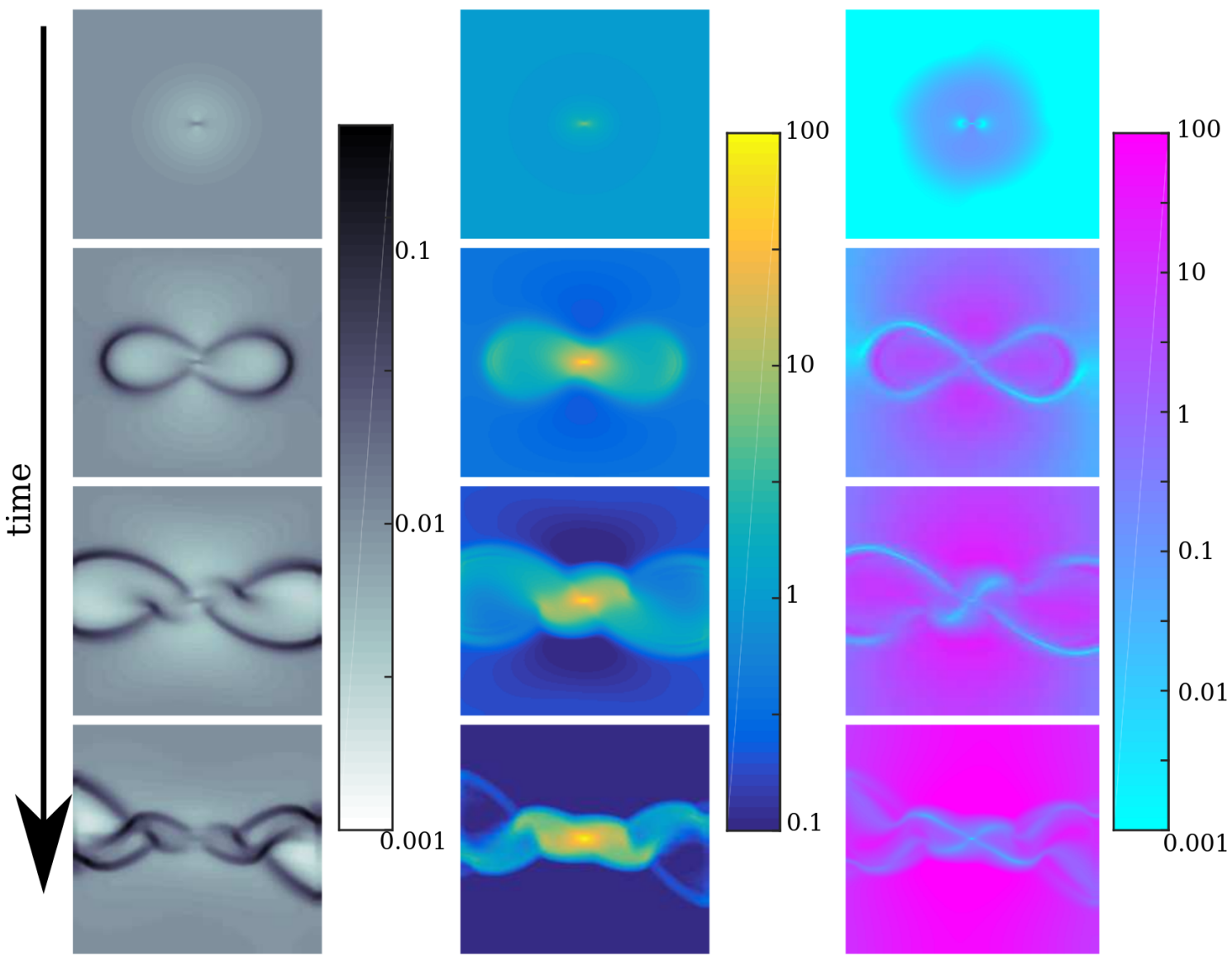

Figure 8.10: Evolution of a 2D granular gas under the effect of gravity. The center of the gravitational interaction is placed in the center of the system. The system was initialized with a small positive velocity of $10^{-4} v_{t h, 0}$ in the angular direction. The system has $\varepsilon=0.9$, $\phi=0.01$; in the center $\phi=0.0001$ to avoid a density collapse. Open boundary condition in $x$ - and $y$-direction are used.

of a granular gas under the influence of a central force such as gravity (see also the movie attached under centralForce.avi). To stabilize the system we reduced the density in the center by a factor 100, and, for the sake of generality, we initialized the system with a small angular momentum. We observe a flow of density towards the center and the formation of dense areas. In the center high pressure leads to a violent density growth. These dense areas are pressed out of the center and start rotating under the influence of a small angular momentum. Eventually the dense regions leave the system through the open boundary conditions. The picture is somehow reminiscent of astrophysical pictures of supernovae.

Here we just want to demonstrate the range of applicability of our DNS to the study of granular gases under the influence of central forces. A technical but important limitation of the current setup is the underlying geometry of the grid employed; this causes problems especially with the boundary conditions but also reduces the accuracy and stability of the simulations. Future work will focus on rewriting the hydrodynamical solver to include cylindrical symmetry. We can conclude that the hydrodynamic of problems with cylindri- 
cal symmetry as they appear in central force systems and Taylor-Couette flows could be of future interest of hydrodynamical studies. 


\section{Conclusions}

Granular materials are collections of macroscopic particles which are present all around us and apart from water they are generally the form of most row materials used in industry, and they are often at the beginning of a production line whether it is in the agriculture or in the plastic industry. The physics of the granular state is fascinating not only because of the ubiquity of it, but also because a wide range of effects can be observed within granular systems which are fundamentally different from other substances, for example arching or vaulting, segregation and pattern formation. However, we can also find states which are analogues of ordinary solid, liquid and gas phases. In this thesis we have mainly studied the granular gas phase and its nonequilibrium transition into dense filamentary clusters within the freely cooling process. We have focused on a hydrodynamical study on granular gases especially within the Navier-Stokes approach. In Chapter 2 we began by introducing the Knudsen number $\mathcal{K} \equiv \frac{\lambda}{L}$, which is the ratio between the mean free path and any characteristic length within the system, as the important number to characterize a hydrodynamic limit. This limit is achieved if $\mathcal{K} \ll 1$. Typically, a granular gas is characterized by Knudsen numbers $10^{-3}<\mathcal{K}<10^{-1}$, thus a hydrodynamic treatment is not unreasonable.

The hydrodynamic theory of granular gases is described in Chapter 2. We described how to derive to the Navier-Stokes equation from the Boltzmann equation in the context of the Chapman-Enskog approach. Two models for the coefficient of restitution have been discussed: the model of a constant coefficient of restitution and the more realistic model of a variable coefficient of restitution, where particles behave like viscoelastic spheres. We have presented two hydrodynamic implementations of the coefficient of restitution in the viscoelastic spheres model: one directly achieved from the kinetic theory for viscoelastic spheres interaction and the second from combining a field theory of the viscoelastic spheres interaction with the Navier-Stokes equations; both models result in slightly different models for the transport coefficients. A comparison has not been reported in the literature so far and we can conclude that we could not observe any qualitative nor quantitative differences between the two models. 
Haff's law as the solution of an ideal, homogeneous, freely cooling granular gas has been presented. The reader was introduced to the linear stability analysis of freely cooling granular gases that reveals the density instability which in turn leads to clustering in granular gases.

In Chapter 3 we have derived the quasi-conservative dimensionless Navier-Stokes equations for granular gases. Luding's pair correlation function is presented to achieve a more realistic model at high filling fractions in $2 \mathrm{D}$. We derive the Prandtl $\mathcal{P}$, Reynolds $\mathcal{R}$ and the Mach $\mathcal{M}$ numbers for granular gases and discuss their behaviors in a granular system. We give a Taylor series of $\mathcal{P}$ and $\mathcal{R}$ in terms of $(1-\varepsilon)$, that is the corrections to the elastic limit.

In Chapter 4 we have shown how to setup state-of-the-art algorithm to solve the granular hydrodynamics equations. Our solver is based on a finite volume method, written in compute unified device architecture and fully implemented on graphics processing unit. We have introduced in detail our numerical solver and showed the use of the main state-of-theart methods: weighted essentially non-oscillatory scheme, arbitrary high order derivative method and the multi-stage method. By implementing such high robust and accurate methods we were able to build an algorithm which is able to simulate extreme situations like we demonstrated in Chapter 8; we can capture strong shocks, high gradients and a wide range of orders of magnitude in the physical observables, which to the best our knowledge has not been achieved so far for granular hydrodynamics.

Theoretical calculations and direct numerical simulations of the granular Navier-Stokes equations in Chapter 5 show that irrespective of the coefficient of restitution, density or initial velocity distribution, the density fluctuations follow a universal quadratic dependence on the system's Mach number. Our findings are substantiate by analytical arguments. We have found that the clustering exhibits a scale-free dynamics but the clustered state becomes observable when the Mach number is approximately of $\mathcal{O}(1)$. Our results provide a method to determine the age of a granular gas and predict the macroscopic appearance of clusters.

We have revealed in Chapter 6 that density fluctuations in a freely cooling granular gas do lead to a temperature drop but not to a drop in the pressure. The drop in the pressure is expected and commonly used to explain the positive feedback of the density fluctuations which eventually lead to a macroscopic clustering. We have demonstrated the influence of subsonic convective flows on the onset of clustering and showed that supersonic shock waves do not appear in the inhomogeneous cooling state but are the only reason for the transition from a homogeneous cooling state to the inhomogeneous cooling state. We could prove, by performing direct numerical simulations and molecular dynamics simulations as well as deriving analytical arguments from the linear stability analysis, that the common heuristic used to explain the onset of clustering does not hold. Our results lead to a different understanding of the clustering in granular gases and might be important whenever a system can be described in the framework of granular gases. It might also lead to a prediction of the lifetime of protoplanetary accretion discs, that is 
their disappearance when macroscopic clustering takes place. In the geophysical context, our results could help improve models of pyroplastic density currents [4].

In Chapter 7 we have shown the finite-size character of the decaying of clusters in a freely cooling granular gas of viscoelastic spheres. We clarified that the disappearing of density fluctuations does depend only on the size of the system. The decay time follows such a strong power law, $t^{\prime} \sim L^{12}$, that only in systems close to the initial critical system size it is realistically possible to observe both the clustering and declustering transitions. Thus, for any amount of dissipation $\left(1-\varepsilon^{2}\right)$ in the system, one can find a system size at which the clusters will appear as stable for an exceedingly long time. On the other hand, our results show that close to $\frac{L}{L_{c r i t}} \gtrsim 1$ the degree of clustering is extremely week (see Fig. 7.2), which poses the challenge to find a system size that is large enough to observe the transition from HCS to ICS, but also small enough to observe the second transition (from ICS back to HCS) on a realistic time scale. Finally, the results of Chapter 7 indicate that an infinitely large system will not decluster.

In Chapter 8 we have shown the distribution of density, velocity and granular temperature in the homogeneous cooling state and the inhomogeneous cooling state. We observe a Gaussian like distribution for the velocity in the homogeneous cooling state, however even in the homogeneous cooling state, density and temperature are not Gaussian distributed but anti-correlated. In the inhomogeneous cooling state distributions of density, velocity and granular temperature are all non-Gaussian. Interestingly, the velocity distribution in the inhomogeneous cooling state looks like the single particle velocity distribution in the homogeneous cooling state, which means that we observe a Gaussian-like behavior around the mean velocity but long tails far away from the mean; this raises the question if the clusters in the inhomogeneous cooling state behave themself like quasi particles.

In Chapter 8 we also had a first look at more realistic models of $\varepsilon$, where $\varepsilon$ follows a probability distribution instead of being a fixed number. We have studied three kinds of distribution and find that the mean value of $\varepsilon$ is a robust predictor of the behavior of the granular gas. These results lend confidence that the model of a constant coefficient of restitution leads to the correct results. We see a deviation from this behavior only for values of $\varepsilon$ very close to unity, where a closer look reveals that the deviation of the $\varepsilon$ becomes more important.

The study of direct numerical simulation of vibrated granular systems reveals enlightening insights into the nonequilibrium collective states of the system. From experiments and molecular dynamics simulations we know of different observable effects namely: the bouncing bed, convection rolls, Leidenfrost effect and undulations. Here we have presented, to the best of our knowledge, for the first time the presence of convection rolls and the Leidenfrost effect in the hydrodynamic model of driven granular systems. We have presented a phase diagram of the different states and find very good agreement with the results of earlier experiments. An important point is that we cannot observe the undulations in our hydrodynamic model, which gives rise to the argument that undulations are not a hydrodynamic effect. Granular matter, in general, and granular gases, specifically, 
are a paradigmatic example of nonequilibrium system. It is our belief that their study is relevant not only for the applications described in the Introduction, but also for what they have to teach about many-body systems far from equilibrium. 


\section{Bibliography}

[1] P. Eshuis, K. Van Der Weele, D. Van Der Meer, R. Bos, and D. Lohse, Phase diagram of vertically shaken granular matter, Physics of Fluids (1994-present) 19, 123301 (2007).

[2] I. S. Aranson and L. S. Tsimring, Granular patterns, (Oxford University Press 2009).

[3] I. Goldhirsch, Rapid granular flows, Annu. Rev. Fluid. Mech. 35, 267 (2003).

[4] J. Dufek, The fluid mechanics of pyroclastic flows, Annu. Rev. Fluid. Mech. 48, 459 (2016).

[5] G. B. Field, Thermal instability., Astrophys. J. 142, 531 (1965).

[6] P. Goldreich and S. Tremaine, The dynamics of planetary rings, Annu. Rev. Astron. Astr. 20, 249 (1982).

[7] F. G. Bridges, A. Hatzes, and D. N. C. Lin, Structure, stability and evolution of Saturn's rings, Nature 309, 333 (1984).

[8] A. Johansen and A. Youdin, Protoplanetary disk turbulence driven by the streaming instability: nonlinear saturation and particle concentration, Astrophys. J. 662, 627 (2007).

[9] S. F. Shandarin and Y. B. Zeldovich, The large-scale structure of the universe: Turbulence, intermittency, structures in a self-gravitating medium, Rev. Mod. Phys. 61, 185 (1989).

[10] E. Falcon, R. Wunenburger, P. Évesque, S. Fauve, C. Chabot, Y. Garrabos, and D. Beysens, Cluster formation in a granular medium fluidized by vibrations in low gravity, Phys. Rev. Lett. 83, 440 (1999).

[11] C. C. Maaß, N. Isert, G. Maret, and C. M. Aegerter, Experimental investigation of the freely cooling granular gas, Phys. Rev. Lett. 100, 248001 (2008).

[12] D. Heißelmann, J. Blum, H. J. Fraser, and K. Wolling, Microgravity experiments on the collisional behavior of saturnian ring particles, Icarus 206, 424 (2010).

[13] A. Sack, M. Heckel, J. E. Kollmer, F. Zimber, and T. Pöschel, Energy dissipation in driven granular matter in the absence of gravity, Phys. Rev. Lett. 111, 018001 (2013). 
[14] K. Harth, T. Trittel, K. May, S. Wegner, and R. Stannarius, Three-dimensional (3d) experimental realization and observation of a granular gas in microgravity, Adv. Space Res. 55, 1901 (2015).

[15] K. Harth, U. Kornek, T. Trittel, U. Strachauer, S. Höme, K. Will, and R. Stannarius, Granular gases of rod-shaped grains in microgravity, Phys. Rev. Lett. 110, 144102 (2013).

[16] J. J. Brey, J. W. Dufty, and A. Santos, Dissipative dynamics for hard spheres, J. Stat. Phys. 87, 1051 (1997).

[17] J. J. Brey, J. W. Dufty, C. S. Kim, and A. Santos, Hydrodynamics for granular flow at low density, Phys. Rev. E 58, 4638 (1998).

[18] V. Garzó and J. Dufty, Dense fluid transport for inelastic hard spheres, Phys. Rev. E 59, 5895 (1999).

[19] P. Haff, Grain flow as a fluid-mechanical phenomenon, JFM 134, 401 (1983).

[20] N. V. Brilliantov, Hydrodynamics of granular gases of viscoelastic particles, Phil. Trans. R. Soc A 360, 415 (2002).

[21] P. Deltour and J.-L. Barrat, Quantitative study of a freely cooling granular medium, Journal de Physique I 7, 137 (1997).

[22] S. Luding and H. Herrmann, Cluster-growth in freely cooling granular media, Chaos 9, 673 (1999).

[23] S. Miller and S. Luding, Cluster growth in two- and three-dimensional granular gases, Phys. Rev. E 69, 031305 (2004).

[24] S. Luding, Structure and cluster formation in granular media, Pramana 64, 893 (2005).

[25] B. Meerson and A. Puglisi, Towards a continuum theory of clustering in a freely cooling inelastic gas, Europhys. Lett. 70, 478 (2005).

[26] E. Falcon, S. Aumaître, P. Evesque, F. Palencia, C. Lecoutre-Chabot, S. Fauve, D. Beysens, and Y. Garrabos, Collision statistics in a dilute granular gas fluidized by vibrations in low gravity, Europhys. Lett. 74, 830 (2006).

[27] G. Kuwabara and K. Kono, Restitution coefficient in a collision between two spheres, Jpn. J. Appl. Phys. 26, 1230 (1987).

[28] T. Schwager and T. Pöschel, Coefficient of normal restitution of viscous particles and cooling rate of granular gases, Phys. Rev. E 57, 650 (1998). 
[29] R. Ramírez, T. Pöschel, N. V. Brilliantov, and T. Schwager, Coefficient of restitution of colliding viscoelastic spheres, Phys. Rev. E 60, 4465 (1999).

[30] N. V. Brilliantov and T. Pöschel, Kinetic Theory of Granular Gases, (Oxford University Press 2004).

[31] M. Babic, Average balance equations for granular materials, Int. J. Engng. Sci. 35, 523 (1997).

[32] I. Goldhirsch, Stress, stress asymmetry and couple stress: from discrete particles to continuous fields, Granul. Matter 12, 239 (2010).

[33] T. Weinhart, A. R. Thornton, S. Luding, and O. Bokhove, From discrete particles to continuum fields near a boundary, Granul. Matter 14, 289 (2012).

[34] I. Goldhirsch and G. Zanetti, Clustering instability in dissipative gases, Phys. Rev. Lett. 70, 1619 (1993).

[35] S. McNamara, Hydrodynamic modes of a uniform granular medium, Physics of Fluids A 5, 3056 (1993).

[36] E. Efrati, E. Livne, and B. Meerson, Hydrodynamic singularities and clustering in a freely cooling inelastic gas, Phys. Rev. Lett. 94, 088001 (2005).

[37] I. Fouxon, B. Meerson, M. Assaf, and E. Livne, Formation and evolution of density singularities in hydrodynamics of inelastic gases, Phys. Rev. E 75, 050301 (2007).

[38] I. Fouxon, B. Meerson, M. Assaf, and E. Livne, Formation of density singularities in ideal hydrodynamics of freely cooling inelastic gases: A family of exact solutions, Phys. Fluids 19, 093303 (2007).

[39] N. Brilliantov, C. Salueña, T. Schwager, and T. Pöschel, Transient structures in a granular gas, Phys. Rev. Lett. 93, 134301 (2004).

[40] V. Becker, T. Schwager, and T. Pöschel, Coefficient of tangential restitution for the linear dashpot model, Physical Review E 77, 011304 (2008).

[41] T. Schwager, V. Becker, and T. Pöschel, Coefficient of tangential restitution for viscoelastic spheres, The European Physical Journal E 27, 107 (2008).

[42] T. T. et al., , Kiron 49-441B, 1020 (in Japanese) (1983).

[43] Y. Taguchi, Powder turbulence: direct onset of turbulent flow, Journal de physique II 2, 2103 (1992).

[44] A. P. Hatzes, F. G. Bridges, and D. Lin, Collisional properties of ice spheres at low impact velocities, Monthly Notices of the Royal Astronomical Society 231, 1091 (1988). 
[45] T. Schwager, Coefficient of restitution for viscoelastic disks, Physical Review E 75, 051305 (2007).

[46] J. P. Clewett, K. Roeller, R. M. Bowley, S. Herminghaus, and M. R. Swift, Emergent surface tension in vibrated, noncohesive granular media, Physical review letters 109, 228002 (2012).

[47] P. Eshuis, K. van der Weele, M. Alam, H. J. van Gerner, M. van der Hoef, H. Kuipers, S. Luding, D. van der Meer, and D. Lohse, Buoyancy driven convection in vertically shaken granular matter: experiment, numerics, and theory, Granular matter 15, 893 (2013).

[48] S. R. Waitukaitis, V. Lee, J. M. Pierson, S. L. Forman, and H. M. Jaeger, Sizedependent same-material tribocharging in insulating grains, Physical Review Letters 112, 218001 (2014).

[49] M. Montaine, M. Heckel, C. Kruelle, T. Schwager, and T. Pöschel, Coefficient of restitution as a fluctuating quantity, Physical Review E 84, 041306 (2011).

[50] H. Struchtrup, Macroscopic transport equations for rarefied gas flows, (Springer 2005).

[51] N. V. Brilliantov and T. Pöschel, Velocity distribution in granular gases of viscoelastic particles, Physical Review E 61, 5573 (2000).

[52] J.-P. Hansen and I. R. McDonald, Theory of Simple Liquids: With Applications to Soft Matter, (Academic Press 2013).

[53] S. Chapman and T. G. Cowling, The mathematical theory of non-uniform gases: an account of the kinetic theory of viscosity, thermal conduction and diffusion in gases, (Cambridge university press 1970).

[54] T. Van Noije and M. Ernst, Velocity distributions in homogeneous granular fluids: the free and the heated case, Granular Matter 1, 57 (1998).

[55] A. Goldshtein and M. Shapiro, Mechanics of collisional motion of granular materials. part 1. general hydrodynamic equations, Journal of Fluid Mechanics 282, 75 (1995).

[56] J. J. Brey and D. Cubero, Hydrodynamic transport coefficients of granular gases, Lecture Notes in Physics 564, 59 (2001).

[57] N. Brilliantov and T. Pöschel, Hydrodynamics and transport coefficients for dilute granular gases, Phys. Rev. E 67, 061304 (2003).

[58] V. Garzó, Grad's moment method for a granular fluid at moderate densities: Navierstokes transport coefficients, Physics of Fluids (1994-present) 25, 043301 (2013). 
[59] N. F. Carnahan and K. E. Starling, Equation of state for nonattracting rigid spheres, J. Chem. Phys. 51, 635 (1969).

[60] S. Luding, Global equation of state of two-dimensional hard sphere systems, Phys. Rev. E 63, 042201 (2001).

[61] S. Luding, Towards dense, realistic granular media in 2d, Nonlinearity 22, R101 (2009).

[62] J. W. Dufty, Fourier's law for a granular fluid, J. Phys. Chem. C 111, 15605 (2007).

[63] M. Hummel, J. P. Clewett, and M. G. Mazza, A universal scaling law for the evolution of granular gases, EPL (Europhysics Letters) 114, 10002 (2016).

[64] C. T. Liu X.-D. Osher S., Weighted essentially non-oscillatory schemes, J. Comput. Phys. 115, 200 (1994).

[65] G.-S. Jiang and C.-W. Shu, Efficient implementation of weighted eno schemes, J. Comput. Phys. 126, 202 (1996).

[66] V. A. Titarev and E. F. Toro, Finite-volume weno schemes for three-dimensional conservation laws, J. Comput. Phys. 201, 238 (2004).

[67] E. F. Toro and V. A. Titarev, Tvd fluxes for the high-order ader schemes, Journal of Scientific Computing 24, 285 (2005).

[68] T. Preis, P. Virnau, W. Paul, and J. J. Schneider, Gpu accelerated monte carlo simulation of the 2d and $3 d$ ising model, Journal of Computational Physics 228, 4468 (2009).

[69] G. Strang, On the construction and comparison of difference schemes, SIAM Journal on Numerical Analysis 5, 506 (1968).

[70] A. Chertock, A. Kurganov, and G. Petrova, Fast explicit operator splitting method for convection-diffusion equations, International Journal for Numerical Methods in Fluids 59, 309 (2009).

[71] H. Holden, K. H. Karlsen, K.-A. Lie, and N. H. Risebro, Splitting methods for partial differential equations with rough solutions, (European Mathematical Society 2010).

[72] E. F. Toro, Riemann solvers and numerical methods for fluid dynamics: a practical introduction, (Springer Science \& Business Media 2009).

[73] J. Stoer and R. Bulirsch, Introduction to numerical analysis, vol. 12, (Springer Science \& Business Media 2013).

[74] J. Shi, C. Hu, and C.-W. Shu, A technique of treating negative weights in weno schemes, Journal of Computational Physics 175, 108 (2002). 
[75] D. Levy, G. Puppo, and G. Russo, A fourth-order central weno scheme for multidimensional hyperbolic systems of conservation laws, SIAM Journal on scientific computing 24, 480 (2002).

[76] D. Levy, G. Puppo, and G. Russo, Central weno schemes for hyperbolic systems of conservation laws, ESAIM: Mathematical Modelling and Numerical Analysis 33, 547 (1999).

[77] J. Qiu and C.-W. Shu, On the construction, comparison, and local characteristic decomposition for high-order central weno schemes, Journal of Computational Physics 183, 187 (2002).

[78] Y. Takakura and E. F. Toro, Arbitarily accurate non-oscillatory schemes for a nonlinear conservation law, Computational Fluid Dynamics Journal 11, 6 (2002).

[79] V. A. Titarev and E. F. Toro, Ader: Arbitrary high order godunov approach, Journal of Scientific Computing 17, 609 (2002).

[80] V. Titarev and E. Toro, Ader schemes for three-dimensional non-linear hyperbolic systems, Journal of Computational Physics 204, 715 (2005).

[81] E. Toro and V. Titarev, Ader schemes for scalar non-linear hyperbolic conservation laws with source terms in three-space dimensions, Journal of Computational Physics 202, 196 (2005).

[82] A. Hidalgo and M. Dumbser, Ader schemes for nonlinear systems of stiff advectiondiffusion-reaction equations, Journal of Scientific Computing 48, 173 (2011).

[83] S. K. Godunov, A difference method for numerical calculation of discontinuous solutions of the equations of hydrodynamics, Matematicheskii Sbornik 89, 271, (in russian) (1959).

[84] P. L. Roe, Approximate riemann solvers, parameter vectors, and difference schemes, Journal of computational physics 43, 357 (1981).

[85] B. Engquist and S. Osher, One-sided difference approximations for nonlinear conservation laws, Mathematics of Computation 36, 321 (1981).

[86] S. Osher and S. Chakravarthy, Upwind schemes and boundary conditions with applications to euler equations in general geometries, Journal of Computational Physics 50, 447 (1983).

[87] A. Harten, P. D. Lax, and B. v. Leer, On upstream differencing and godunov-type schemes for hyperbolic conservation laws, SIAM review 25, 35 (1983).

[88] B. Einfeldt, On godunov-type methods for gas dynamics, SIAM Journal on Numerical Analysis 25, 294 (1988). 
[89] B. Einfeldt, C.-D. Munz, P. L. Roe, and B. Sjögreen, On godunov-type methods near low densities, Journal of computational physics 92, 273 (1991).

[90] E. F. Toro, M. Spruce, and W. Speares, Restoration of the contact surface in the hll-riemann solver, Shock waves 4, 25 (1994).

[91] E. Toro, On glimm-related schemes for conservation laws, Preprint MMU-9602, Department of $1 \backslash /$ Iathematics and Physics, Manchester $l \backslash /$ Ietropolitan University, UK (1996).

[92] E. F. Toro, Musta: A multi-stage numerical flux, Applied Numerical Mathematics 56, 1464 (2006).

[93] G.-Q. Chen and E. F. Toro, Centered difference schemes for nonlinear hyperbolic equations, Journal of hyperbolic differential equations 1, 531 (2004).

[94] E. Toro, Riemann solvers with evolved initial conditions, International journal for numerical methods in fluids 52, 433 (2006).

[95] E. F. Toro and V. A. Titarev, Musta fluxes for systems of conservation laws, Journal of Computational Physics 216, 403 (2006).

[96] W. H. Press, S. A. Teukolsky, W. T. Vetterling, and B. P. Flannery, Numerical Recipes 3rd Edition: The Art of Scientific Computing, (Cambridge University Press, New York, NY, USA 2007), 3 ed.

[97] G. Gassner, F. Lörcher, and C.-D. Munz, A contribution to the construction of diffusion fluxes for finite volume and discontinuous galerkin schemes, Journal of Computational Physics 224, 1049 (2007).

[98] F. Lörcher, G. Gassner, and C.-D. Munz, An explicit discontinuous galerkin scheme with local time-stepping for general unsteady diffusion equations, Journal of Computational Physics 227, 5649 (2008).

[99] B. Meerson, I. Fouxon, and A. Vilenkin, Nonlinear theory of nonstationary low Mach number channel flows of freely cooling nearly elastic granular gases, Phys. Rev. E 77, 021307 (2008).

[100] A. Puglisi, M. Assaf, I. Fouxon, and B. Meerson, Attempted density blowup in a freely cooling dilute granular gas: Hydrodynamics versus molecular dynamics, Phys. Rev. E 77, 021305 (2008).

[101] I. Kolvin, E. Livne, and B. Meerson, Navier-Stokes hydrodynamics of thermal collapse in a freely cooling granular gas, Phys. Rev. E 82, 021302 (2010).

[102] S. R. Ahmad and S. Puri, Velocity distributions and aging in a cooling granular gas, Phys. Rev. E 75, 031302 (2007). 
[103] I. S. Aranson and L. S. Tsimring, Patterns and collective behavior in granular media: Theoretical concepts, Rev. Mod. Phys. 78, 641 (2006).

[104] S. N. Pathak, Z. Jabeen, D. Das, and R. Rajesh, Energy decay in three-dimensional freely cooling granular gas, Phys. Rev. Lett. 112, 038001 (2014).

[105] M. Hummel and M. G. Mazza, Declustering in a granular gas as a finite-size effect, Physical Review E 93, 022905 (2016).

[106] S. González and S. Luding, Consequences of using different pair-correlation functions on the stability properties of the homogeneous cooling state for a monodisperse system of near-elastic disks, The European Physical Journal Special Topics 179, 55 (2009).

[107] S. K. Das and S. Puri, Pattern formation in the inhomogeneous cooling state of granular fluids, EPL (Europhysics Letters) 61, 749 (2003).

[108] E. Ben-Naim, S. Chen, G. Doolen, and S. Redner, Shocklike dynamics of inelastic gases, Physical review letters 83, 4069 (1999).

[109] V. Kamenetsky, A. Goldshtein, M. Shapiro, and D. Degani, Evolution of a shock wave in a granular gas, Physics of Fluids (1994-present) 12, 3036 (2000).

[110] X. Nie, E. Ben-Naim, and S. Chen, Dynamics of freely cooling granular gases, Phys. Rev. Lett. 89, 204301 (2002).

[111] T. Pöschel, N. V. Brilliantov, and T. Schwager, Long-time behavior of granular gases with impact-velocity dependent coefficient of restitution, Physica A 325, 274 (2003).

[112] M. L. Reddy, S. Ansumali, and M. Alam, Shock waves in a dilute granular gas, in PROCEEDINGS OF THE 29TH INTERNATIONAL SYMPOSIUM ON RAREFIED GAS DYNAMICS, vol. 1628, 480-487, (AIP Publishing 2014).

[113] M. L. Reddy and M. Alam, Plane shock waves and haffâĂŹs law in a granular gas, Journal of Fluid Mechanics 779, R2 (2015).

[114] T. Pöschel and N. V. Brilliantov, Granular gas dynamics, vol. 624, (Springer Science \& Business Media 2003).

[115] T. Pöschel, N. V. Brilliantov, and T. Schwager, Transient clusters in granular gases, J. Phys.: Condens. Matter 17, S2705 (2005).

[116] A. Bodrova and N. Brilliantov, Granular gas of viscoelastic particles in a homogeneous cooling state, Physica A: Statistical Mechanics and its Applications 388, 3315 (2009).

[117] S. E. Esipov and T. Pöschel, The granular phase diagram, Journal of statistical physics 86, 1385 (1997). 
[118] I. N. Bronshtein and K. A. Semendyayev, Handbook of mathematics, (Springer Science \& Business Media 2013).

[119] M. Herr, The generation of a homogeneous, steady state in granular gases from inhomogeneous state, Master's thesis, Georg-August-University Goettingen (2015).

[120] P. Eshuis, K. van der Weele, D. van der Meer, and D. Lohse, Granular leidenfrost effect: Experiment and theory of floating particle clusters, Physical review letters 95, 258001 (2005).

[121] J. Tabet, Hydrodynamic simulation on driven granular media (unfinished at the current state), Master's thesis, Georg-August-University Goettingen (2016).

[122] A. Glielmo, N. Gunkelmann, and T. Pöschel, Coefficient of restitution of aspherical particles, Physical Review E 90, 052204 (2014). 


\section{Appendix}

\subsection{Eigenvalues of the linearized set of Navier-Stokes equation}

We give here the eigenvalues for the matrix $\underline{\mathbf{M}}$ in Eq. (2.87) which was derived for the linear stability analysis of the homogeneous cooling state in section 2.9. The eigenvalues read as

$$
\begin{aligned}
& \lambda_{1}=-\frac{1}{3}\left[\varkappa_{1}+\left(\frac{\varkappa_{0}}{2}\right)^{1 / 3}-\left(3 \varkappa_{2}-\varkappa_{1}^{2}\right)\left(\frac{\varkappa_{0}}{2}\right)^{-1 / 3}\right], \\
& \lambda_{2}=-\frac{1}{3}\left[\varkappa_{1}-\left(\frac{1-\mathrm{i} \sqrt{3}}{2}\right)\left(\frac{\varkappa_{0}}{2}\right)^{1 / 3}+\left(\frac{1+\mathrm{i} \sqrt{3}}{2}\right)\left(3 \varkappa_{2}-\varkappa_{1}^{2}\right)\left(\frac{\varkappa_{0}}{2}\right)^{-1 / 3}\right], \\
& \lambda_{3}=-\frac{1}{3}\left[\varkappa_{1}-\left(\frac{1+\mathrm{i} \sqrt{3}}{2}\right)\left(\frac{\varkappa_{0}}{2}\right)^{1 / 3}+\left(\frac{1-\mathrm{i} \sqrt{3}}{2}\right)\left(3 \varkappa_{2}-\varkappa_{1}^{2}\right)\left(\frac{\varkappa_{0}}{2}\right)^{-1 / 3}\right] .
\end{aligned}
$$

where

$$
\begin{aligned}
& \varkappa_{0}=2 \varkappa_{1}^{3}-9 \varkappa_{1} \varkappa_{2}-27 \varkappa_{3}+\left\{4\left(3 \varkappa_{2}-\varkappa_{1}^{2}\right)^{3}+\left(2 \varkappa_{1}^{3}-9 \varkappa_{1} \varkappa_{2}-27 \varkappa_{3}\right)^{2}\right\}^{1 / 2}, \\
& \varkappa_{1}=\frac{1}{\tilde{C}_{\beta}} k^{2}\left[\frac{2}{d}(d-1) \eta^{*}+\left(\frac{d+2}{d-1}\right) \kappa^{*}\right] \\
& \varkappa_{2}=-\left[\frac{1}{4} \tilde{C}_{\beta} \xi^{*}-\frac{2}{d}(d-1) \frac{1}{\tilde{C}_{\beta}} \eta^{*} k^{2}\right]\left[\frac{1}{4} \tilde{C}_{\beta} \xi^{*}+\left(\frac{d+2}{d-1}\right) \frac{1}{\tilde{C}_{\beta}} \kappa^{*} k^{2}\right]+\frac{(d+2)}{2 d} k^{2}, \\
& \varkappa_{3}=\frac{1}{2} k^{2}\left[\frac{1}{4} \tilde{C}_{\beta} \xi^{*}+\left(\frac{d+2}{d-1}\right) \frac{1}{\tilde{C}_{\beta}}\left(\kappa^{*}-\mu^{*}\right) k^{2}\right] .
\end{aligned}
$$

The eigenvalue $\lambda_{1} \in \mathbb{R}$ is related to the "heat" mode and causes the density instability. The values $\lambda_{2,3}$ are related to the sound mode and $\operatorname{Re}\left(\lambda_{2,3}<0\right)$. A sketch on the eigenvalue for specific $\bar{\phi}$ and $\varepsilon$ is found in Fig. 2.4. 
The eigenvectors corresponding to above eigenvalues — respectively—read

$$
\begin{aligned}
& \boldsymbol{\Phi}_{1}=\left[\begin{array}{llll}
-\frac{\varkappa_{4}+\varkappa_{9}}{\varkappa_{8}}+\frac{k^{2}\left(\varkappa_{4}-\varkappa_{8}+\varkappa_{9}\right)}{\varkappa_{8}\left\{k^{2}+d \varkappa_{8}\left(\varkappa_{4}+\varkappa_{7}\right)\right\}} & -\frac{d}{2} \frac{\mathrm{i} k\left(\varkappa_{4}-\varkappa_{8}+\varkappa_{9}\right)}{\varkappa_{8}\left\{k^{2}+d \varkappa_{8}\left(\varkappa_{4}+\varkappa_{7}\right)\right\}} & 1
\end{array}\right]^{\top} \\
& \boldsymbol{\Phi}_{2}=\left[\begin{array}{llll}
-\frac{\varkappa_{5}+\varkappa_{9}}{\varkappa_{8}}+\frac{k^{2}\left(\varkappa_{5}-\varkappa_{8}+\varkappa_{9}\right)}{\varkappa_{8}\left\{k^{2}+d \varkappa_{8}\left(\varkappa_{5}+\varkappa_{7}\right)\right\}} & -\frac{d}{2} \frac{\mathrm{i} k\left(\varkappa_{5}-\varkappa_{8}+\varkappa_{9}\right)}{\varkappa_{8}\left\{k^{2}+d \varkappa_{8}\left(\varkappa_{5}+\varkappa_{7}\right)\right\}} & 1
\end{array}\right]^{\top} \\
& \boldsymbol{\Phi}_{3}=\left[\begin{array}{llll}
-\frac{\varkappa_{6}+\varkappa_{9}}{\varkappa_{8}}+\frac{k^{2}\left(\varkappa_{6}-\varkappa_{8}+\varkappa_{9}\right)}{\varkappa_{8}\left\{k^{2}+d \varkappa_{8}\left(\varkappa_{6}+\varkappa_{7}\right)\right\}} & -\frac{d}{2} \frac{\mathrm{i} k\left(\varkappa_{6}-\varkappa_{8}+\varkappa_{9}\right)}{\varkappa_{8}\left\{k^{2}+d \varkappa_{8}\left(\varkappa_{6}+\varkappa_{7}\right)\right\}} & 1
\end{array}\right]^{\top}
\end{aligned}
$$

where

$$
\begin{aligned}
& \varkappa_{4}=\frac{\varkappa_{1}}{3}-\frac{\varkappa_{10}}{3 \times 2^{1 / 3}}+\frac{2^{1 / 3}\left(3 \varkappa_{2}-\varkappa_{1}^{2}\right)}{3 \varkappa_{10}}, \\
& \varkappa_{5}=\frac{\varkappa_{1}}{3}+\frac{(1-\mathrm{i} \sqrt{3}) \varkappa_{10}}{6 \times 2^{1 / 3}}-\frac{2^{1 / 3}(1+\mathrm{i} \sqrt{3})\left(3 \varkappa_{2}-\varkappa_{1}^{2}\right)}{6 \varkappa_{10}}, \\
& \varkappa_{6}=\frac{\varkappa_{1}}{3}+\frac{(1+\mathrm{i} \sqrt{3}) \varkappa_{10}}{6 \times 2^{1 / 3}}-\frac{2^{1 / 3}(1-\mathrm{i} \sqrt{3})\left(3 \varkappa_{2}-\varkappa_{1}^{2}\right)}{6 \varkappa_{10}}, \\
& \varkappa_{7}=\frac{1}{4} \tilde{C}_{\beta} \xi^{*}-\frac{2}{d}(d-1) \frac{1}{\tilde{C}_{\beta}} \eta^{*} k^{2}, \\
& \varkappa_{8}=-\frac{1}{2} \tilde{C}_{\beta} \xi^{*}-\left(\frac{d+2}{d-1}\right) \frac{1}{\tilde{C}_{\beta}} \mu^{*} k^{2}, \\
& \varkappa_{9}=-\frac{1}{4} \tilde{C}_{\beta} \xi^{*}-\left(\frac{d+2}{d-1}\right) \frac{1}{\tilde{C}_{\beta}} \kappa^{*} k^{2}, \\
& \varkappa_{10}=\left[\varkappa_{0}-2\left(2 \varkappa_{1}^{3}-9 \varkappa_{1} \varkappa_{2}-27 \varkappa_{3}\right)\right]^{1 / 3} .
\end{aligned}
$$

\subsection{WENO function in C-code}

The following WENO method rebuilds the value on a gaussian integration point $U_{i+\Delta x /(2 \sqrt{3})}$ from average values $\overline{\boldsymbol{U}}_{i-3}, \overline{\boldsymbol{U}}_{i-2}, \overline{\boldsymbol{U}}_{i-1}, \overline{\boldsymbol{U}}_{i}, \overline{\boldsymbol{U}}_{i+1}, \overline{\boldsymbol{U}}_{i+2}, \overline{\boldsymbol{U}}_{i+3}$.

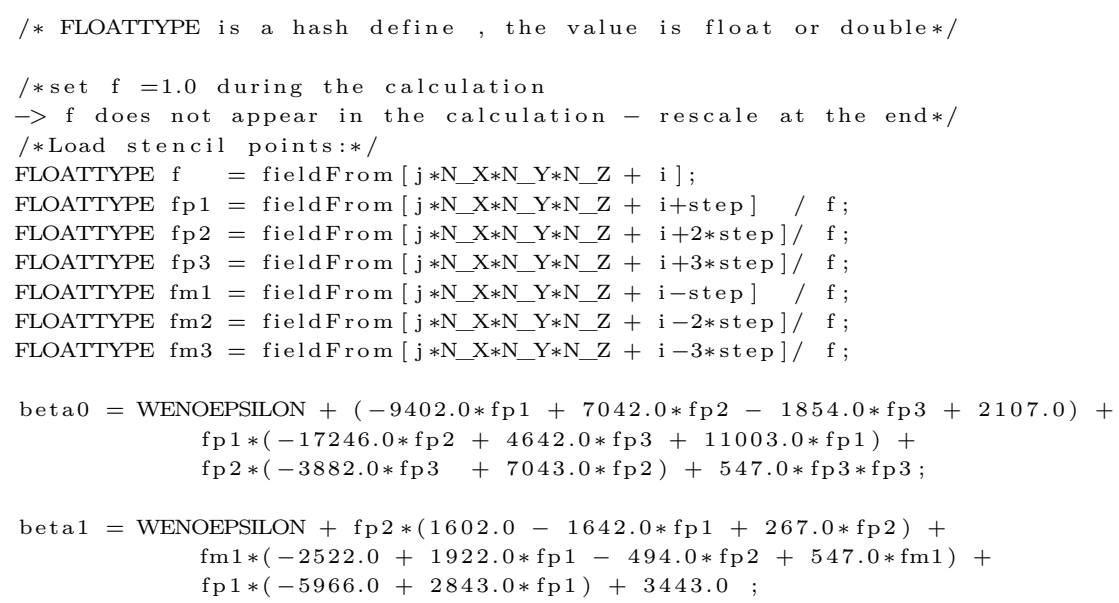




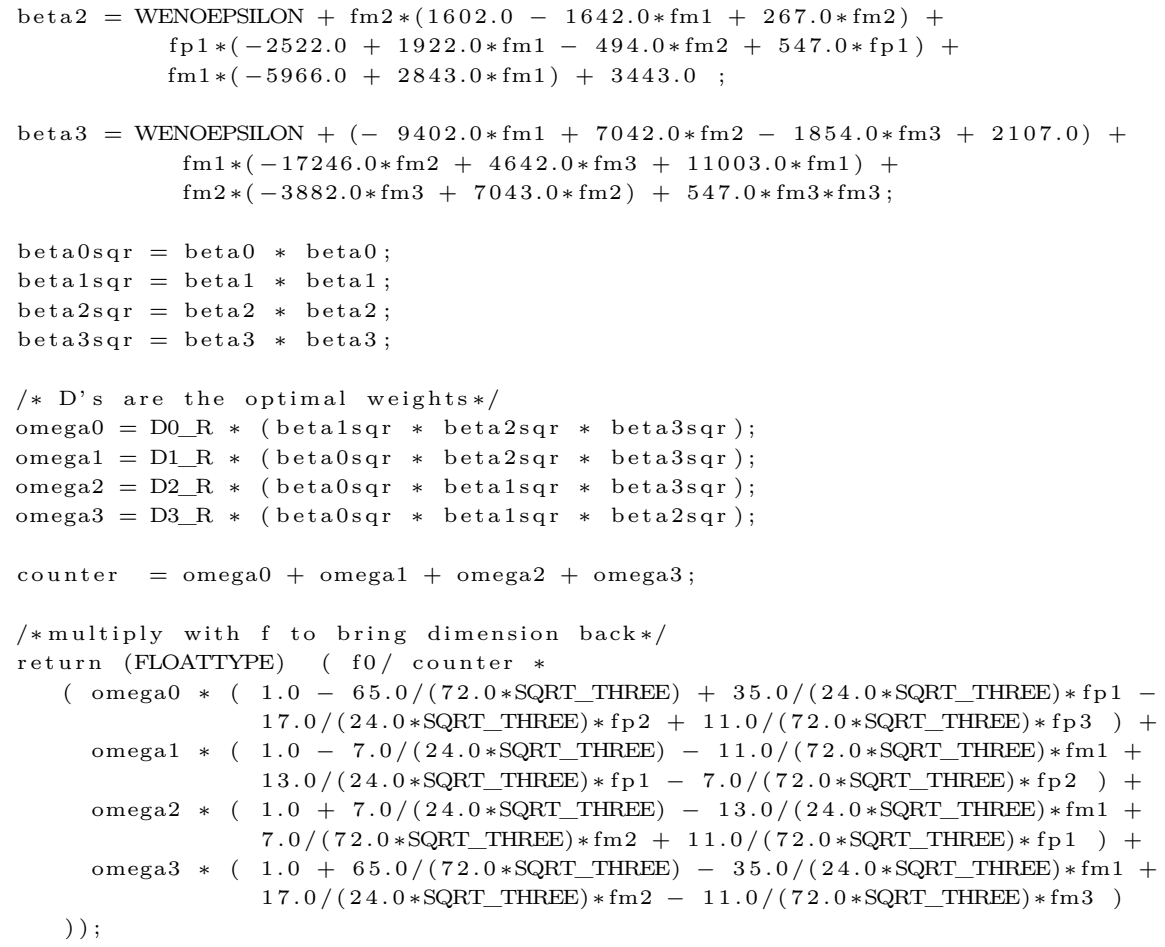

\subsection{Mathematica script for WENO seventh order}

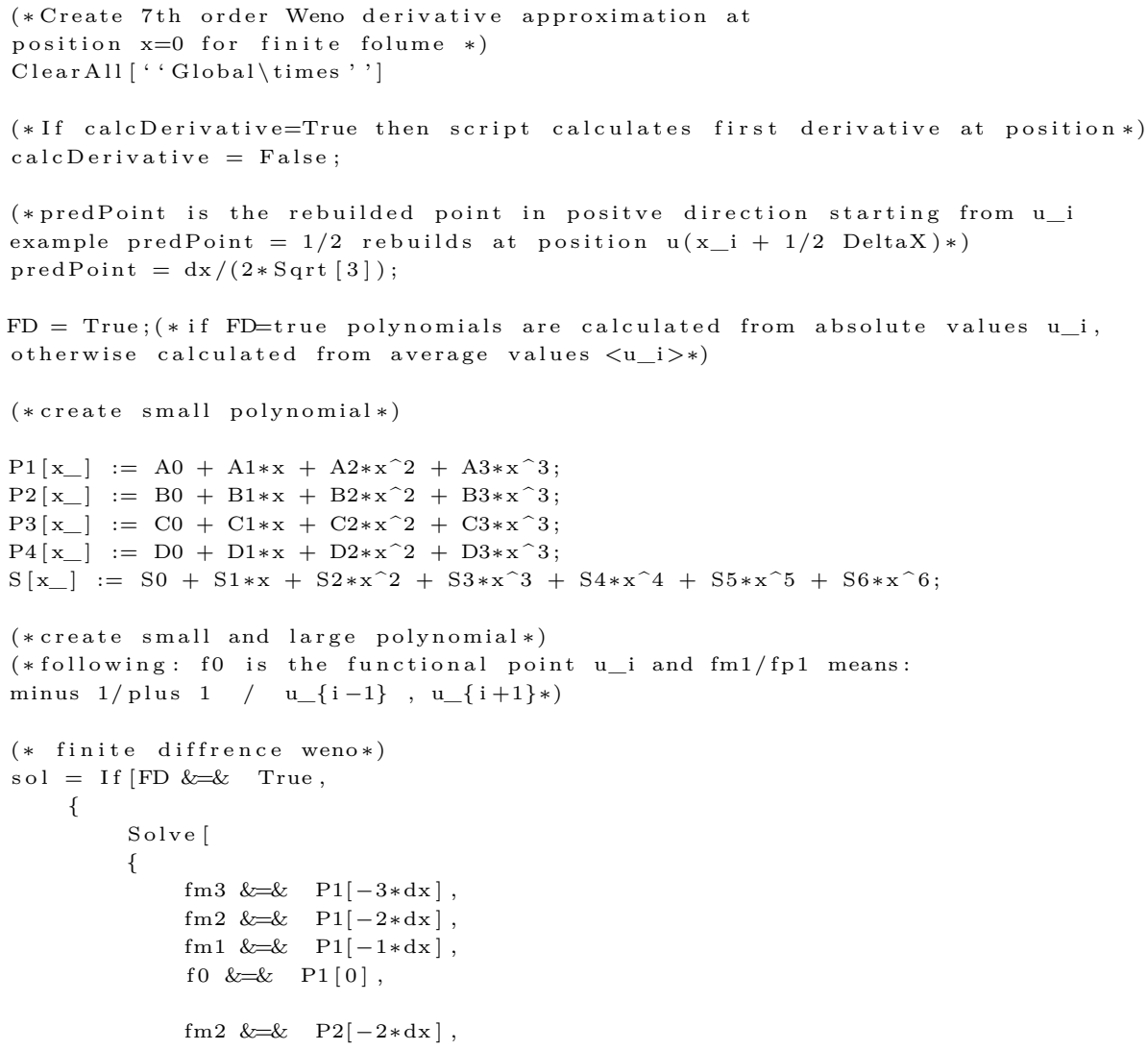




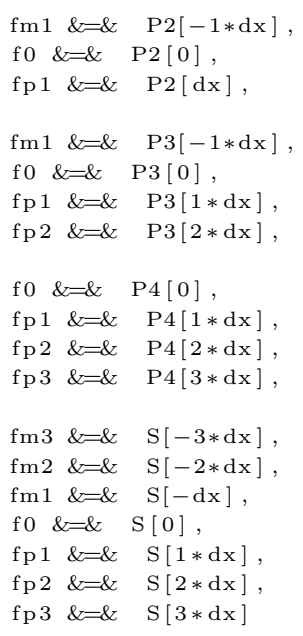

$\{\mathrm{A} 0, \mathrm{~A} 1, \mathrm{~A} 2, \mathrm{~A} 3, \mathrm{~B} 0, \mathrm{~B} 1, \mathrm{~B} 2, \mathrm{~B} 3, \mathrm{C} 0, \mathrm{C} 1, \mathrm{C} 2, \mathrm{C} 3, \mathrm{D} 0, \mathrm{D} 1, \mathrm{D} 2, \mathrm{D} 3$, $\mathrm{S} 0, \mathrm{~S} 1, \mathrm{~S} 2, \mathrm{~S} 3, \mathrm{~S} 4, \mathrm{~S} 5, \mathrm{~S} 6\}$

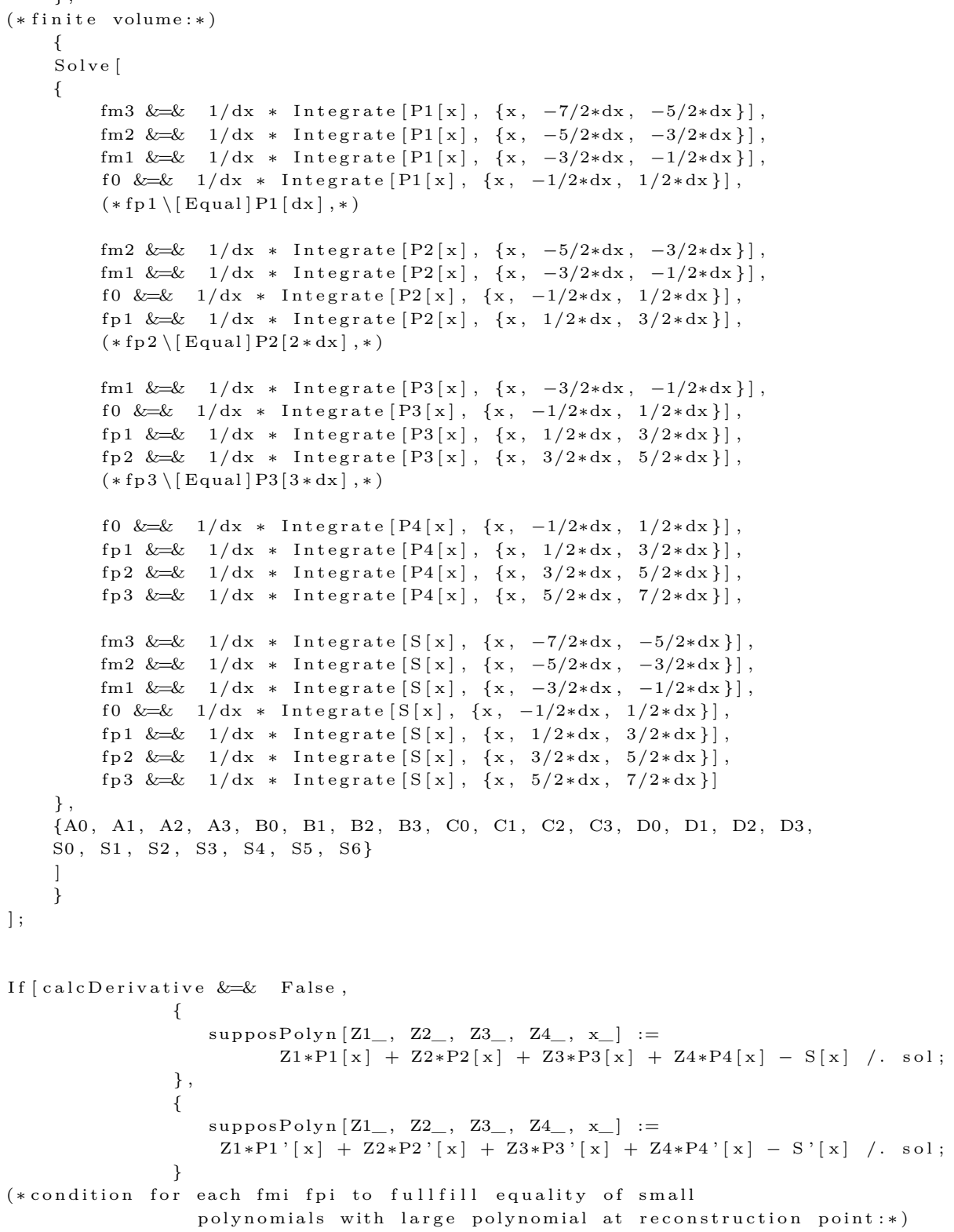


foo = MonomialList [supposPolyn $[\mathrm{Z1}, \mathrm{Z} 2, \mathrm{Z} 3, \mathrm{Z} 4$, predPoint $]$,

$\{\mathrm{fm} 3, \mathrm{fm} 2, \mathrm{fm} 1, \mathrm{f}, \mathrm{fp} 1, \mathrm{fp} 2, \mathrm{fp} 3\}]$;

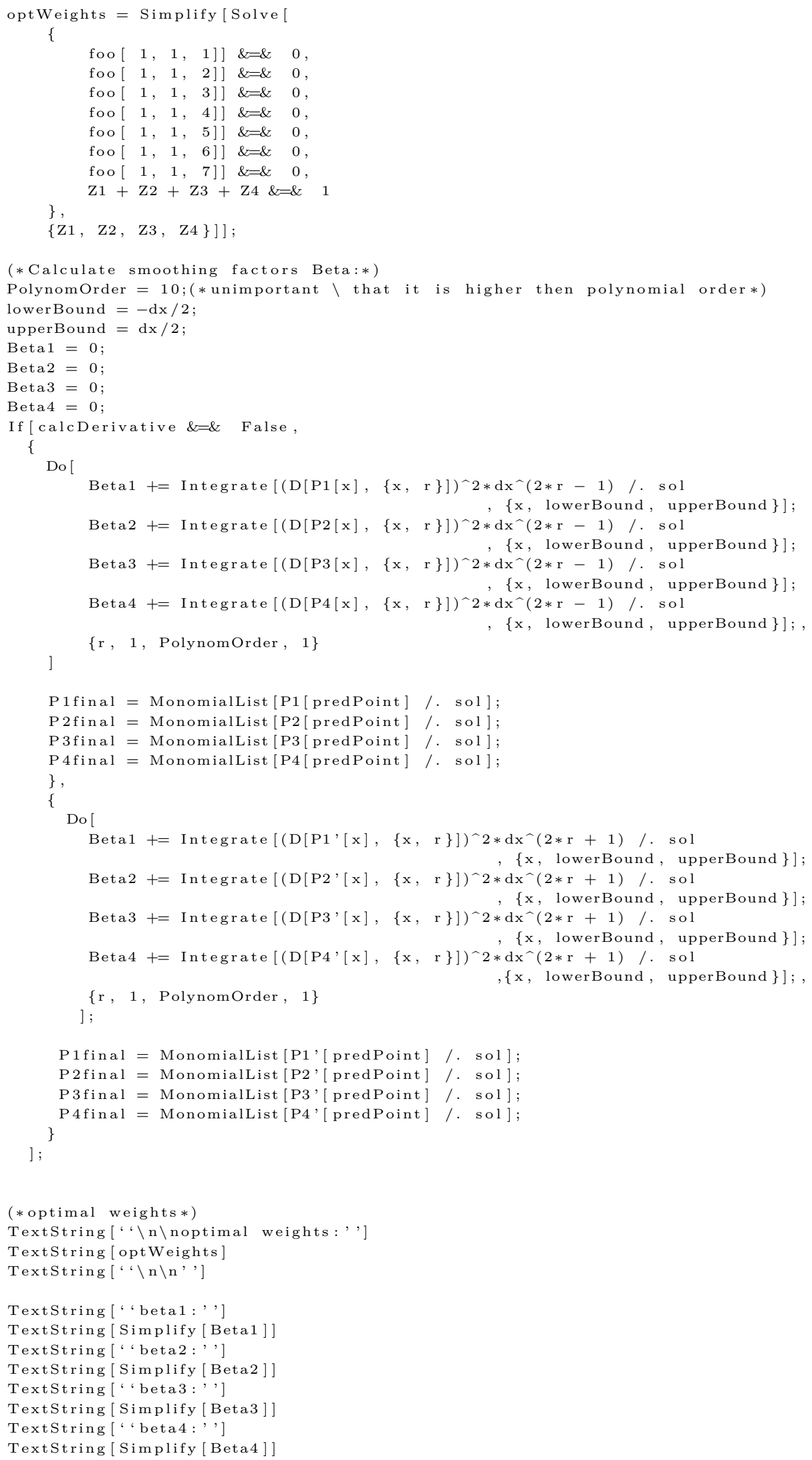




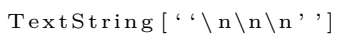

TextString [ ' polynom 1:',]

TextString[P1final[ 1, 1, 1] ] + P1final [1, 1, 2] ] + P1final [ 1, 1, 3] ]

TextString [ 'polynom 2:',]

+ P1final $[1,1,4]]]$

TextString [P2final $[1,1,1]]+\operatorname{P} 2$ final $[1,1,2]]+\operatorname{P} 2$ final $[1,1,3]]$

TextString [ " polynom 3:',]

+ P2final $[1,1,4]]]$

TextString[P3final [1, 1, 1] ] + P 3final $[1,1,2]]+\operatorname{P} 3 f i n a l[1,1,3]]$

TextString ["'polynom 4:',]

+ P3final $[1,1,4]]]$

TextString [P4final [ 1, 1, 1] ] + P4final [1, 1, 2] ] + P4final [ 1, 1, 3] ]

+ P4final $[1,1,4]]]$ 


\title{
Curriculum Vitae
}

\section{Current address}

\author{
Mathias Hummel \\ Eisenacher Str. 17 \\ 37085 Göttingen \\ Germany
}

\section{Personal Information}

Date of Birth: May 23, 1985

Place of Birth: Magdeburg, Germany

\section{Previous education}

1995-2004: Abitur, Albert Einstein Gymnasium, Magdeburg, Germany 2005-2011: German diploma in physics, Otto-von-Guericke-Universität Magdeburg, Magdeburg, Germany

\section{Publications}

M.Hummel, J. P. D. Clewett and M. G. Mazza, A universal scaling law for the evolution of granular gases, Europhysics Letters 114, 10002 (2016)

M.Hummel and M. G. Mazza, Declustering in a granular gas as a finite-size effect, Physiscal Review E 93, 022905 (2016) 
I confirm that I have written this thesis independently and with no other sources and aids than quoted.

Mathias Hummel

Göttingen, September 2016 